\title{
Measurement of Quark Energy Loss in Cold Nuclear Matter at Fermilab E906/SeaQuest
}

\author{
by \\ Po-Ju Lin \\ B.S., National Kaohsiung Normal University, 2004 \\ M.S., National Kaohsiung Normal University, 2006
}

\author{
A thesis submitted to the \\ Faculty of the Graduate School of the \\ University of Colorado in partial fulfillment \\ of the requirements for the degree of \\ Doctor of Philosophy \\ Department of Physics
}

2017 
This thesis entitled:

Measurement of Quark Energy Loss in Cold Nuclear Matter at Fermilab E906/SeaQuest written by Po-Ju Lin

has been approved for the Department of Physics

Prof. Edward R. Kinney

Prof. John P. Cumalat

Dr. Donald F. Geesaman

Prof. Yung Cheng Lee

Prof. James L. Nagle

Prof. Paul Romatschke

Date

The final copy of this thesis has been examined by the signatories, and we find that both the content and the form meet acceptable presentation standards of scholarly work in the above mentioned discipline. 
Lin, Po-Ju (Ph.D., Physics)

Measurement of Quark Energy Loss in Cold Nuclear Matter at Fermilab E906/SeaQuest

Thesis directed by Prof. Edward R. Kinney

Parton energy loss is a process within QCD that draws considerable interest. The measurement of parton energy loss can provide valuable information for other hard-scattering processes in nuclei, and also serves as an important tool for exploring the properties of the quark-gluon plasma (QGP). Quantifying the energy loss in cold nuclear matter will help to set a baseline relative to energy loss in the QGP. With the Drell-Yan process, the energy loss of incoming quarks in cold nuclear matter can be ideally investigated since the final state interaction is expected to be minimal. E906/SeaQuest is a fixed-target experiment using the $120 \mathrm{GeV}$ proton beam from the Fermilab Main Injector and has been collecting data from $\mathrm{p}+\mathrm{p}, \mathrm{p}+\mathrm{d}, \mathrm{p}+\mathrm{C}, \mathrm{p}+\mathrm{Fe}$, and $\mathrm{p}+\mathrm{W}$ collisions. Within the E906 kinematic coverage of Drell-Yan production via the dimuon channel, the quark energy loss can be measured in a regime where other nuclear effects are expected to be small. In this thesis, the study of quark energy loss from different cold nuclear targets is presented. 


\section{Dedication}

To my family. 


\section{Acknowledgements}

I would like to express my sincere gratitude to my advisor, Edward Kinney, who has been patiently guiding me through different stages of my Ph.D. study. I would also like to give my appreciation to Eric Erdos, Joseph Katich, and Brian McDonald, for their companion and help in building the chamber. I want to thank all the SeaQuest Collaborators. It is my pleasure to be able to work with you and I am grateful for the kind assistance I have received. Thank you to the co-spokespersons, Donald Geesaman and Paul Reimer, for their continuing guidance and support. I wish to give my heartfelt gratitude to Charles Brown and David Christian, who taught me their precious experience and knowledge on not only the drift chambers, but also various aspects of the experiment. Many thanks to all the members involved in the chamber work: Kenichi Nakano, Shou Miyasaka, Kei Nagai, Shunpei Nara, and especially Arun Tadepalli. I will never forget those times we spent in Lab 6, scratching our heads together, trying to make the chamber work. I want to thank Jen-Chieh Peng for providing me his insights and advises on the energy loss study. Thank you to Kun Liu for the useful discussion on the analysis. My special thanks go to Wen-Chen Chang, Yen-Chu Chen, and Su-Yin Wang, for their generous support for my stay at Fermilab. Finally, I would like to thank my parents, my brothers, my wife Yung-Chu, my son Ang-Yu, and my unborn daughter, who gave me the warm support and strength to finish this study. 


\section{Contents}

\section{Chapter}

\begin{tabular}{lll}
\hline & Introduction & 1
\end{tabular}

1.1 The Strong Interaction and Quantum Chromodynamics . . . . . . . . . . . . . . 1

1.2 Parton Energy Loss $\ldots \ldots \ldots \ldots \ldots$

1.3 Radiative Energy Loss $\ldots \ldots \ldots \ldots$. . . . . . . . . . . . . . . . 6

$1.3 .1 \quad$ Multiple Gluon Emission $\ldots \ldots \ldots \ldots \ldots \ldots$

1.4 Collisional Energy Loss $\ldots \ldots \ldots \ldots \ldots$

1.5 Experimental Measurements $\ldots \ldots \ldots \ldots \ldots$. . . . . . . . . . . . . . . 14

1.6 The Drell-Yan Interaction $\ldots \ldots \ldots \ldots \ldots \ldots$

1.6 .1 Kinematics of the Drell-Yan Process . . . . . . . . . . . . . . . . . . . . . 19

1.6 .2 Cross Section of the Drell-Yan Process $\ldots \ldots \ldots \ldots$. . . . . . . . . 21

1.6 .3 Higher Order QCD Correction $\ldots \ldots \ldots \ldots$. . . . . . . . . . 23

1.7 Energy Loss Measurements with Drell-Yan $\ldots \ldots \ldots$. . . . . . . . . . . . . . 25

1.7.1 Nuclear Dependence of Parton Distributions _ . . . . . . . . . . . . . 28

1.7 .2 Previous Measurements . . . . . . . . . . . . . . . . . . . . . . 30

1.7 .3 E906/SeaQuest Experiment . . . . . . . . . . . . . . . . 33

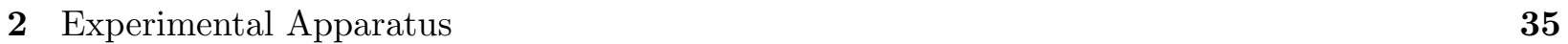

2.1 Beam and Beam Monitors $\ldots \ldots \ldots \ldots \ldots$

$2.1 .1 \quad$ Beam Intensity Monitor $\ldots \ldots \ldots \ldots$. . . . . . . . . . . . . 38 


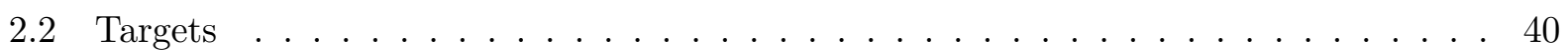

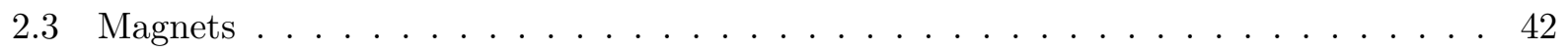

2.4 Tracking Detectors $\ldots \ldots \ldots \ldots \ldots \ldots \ldots$. . . . . . . . . . . . . . . . . . . . . .

2.4 .1 Hodoscopes $\ldots \ldots \ldots \ldots \ldots \ldots$

2.4 .2 Drift Chambers . . . . . . . . . . . . . . . 46

2.4 .3 Proportional Tubes $\ldots \ldots \ldots \ldots \ldots \ldots$

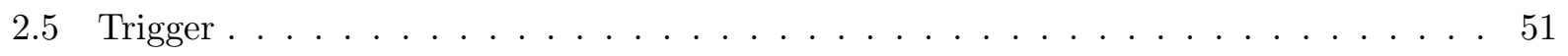

2.6 Data Acquisition and Handling . . . . . . . . . . . . . . . . . . . 53

2.6 .1 Main DAQ $\ldots \ldots \ldots \ldots \ldots \ldots \ldots \ldots \ldots$

$2.6 .2 \quad$ Scaler DAQ $\ldots \ldots \ldots \ldots \ldots \ldots \ldots \ldots$

2.6 .3 Beam DAQ . . . . . . . . . . . . . . . . . . . 57

2.6 .4 Slow Controls . . . . . . . . . . . . . . . . . . 57

2.6 .5 Data Decoding and Storage . . . . . . . . . . . . . . . 58

\begin{tabular}{|ccc}
\hline 3 & Data Analysis & 60
\end{tabular}

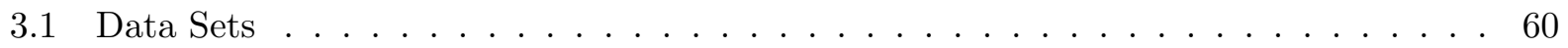

3.2 Track Reconstruction $\ldots \ldots \ldots \ldots \ldots \ldots$

$3.2 .1 \quad$ Pre-tracking analysis $\ldots \ldots \ldots \ldots \ldots \ldots \ldots \ldots$

3.2 .2 Track Reconstruction $\ldots \ldots \ldots \ldots \ldots \ldots$

3.2 .3 Vertex finding . . . . . . . . . . . . . . . . . 67

3.3 Event Selection . . . . . . . . . . . . . . . . . . . . . . . . . . 69

3.3 .1 Track-level Cuts . . . . . . . . . . . . . . . . . 70

$3.3 .2 \quad$ Dimuon-level Cuts and Target Dimuon Selection . . . . . . . . . . . . . . 71

3.4 Monte Carlo Simulation $\ldots \ldots \ldots \ldots \ldots \ldots$

3.5 Per-nucleon Cross Section Ratios $\ldots \ldots \ldots \ldots$. . . . . . . . . . . 75

3.5 .1 Effective Number of Protons Evaluation . . . . . . . . . . . . . . . . 78

3.6 Background $\ldots \ldots \ldots \ldots \ldots \ldots \ldots$ 
3.6.1 Combinatorial Background $\ldots \ldots \ldots \ldots \ldots$. . . . . . . . . . . 79

3.6 .2 Background Shape . . . . . . . . . . . . . . . . . . 81

$3.6 .3 \quad$ Background Normalization $\ldots \ldots \ldots$. . . . . . . . . . . . . . . 90

3.6.4 Normalized Yield Ratios after Background Subtraction . . . . . . . . . . . . . 105

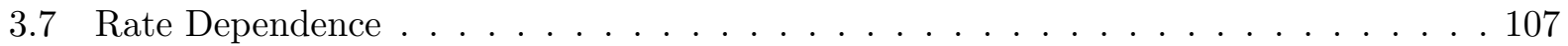

3.7 .1 Reconstruction Efficiency $\ldots \ldots \ldots$. . . . . . . . . . . . . 107

3.7 .2 Kinematic Dependence. . . . . . . . . . . . . . . . . . 111

3.7 .3 Correction for Reconstruction Efficiency . . . . . . . . . . . . . . . . . 120

3.7 .4 Remaining Rate Dependence . . . . . . . . . . . . . . . . . . . . . 128

3.8 Detector Acceptance $\ldots \ldots \ldots \ldots$. . . . . . . . . . . . . . . . . . 131

3.9 Dependency on Other Kinematic Variables $\ldots \ldots \ldots$. . . . . . . . . . . . . . 132

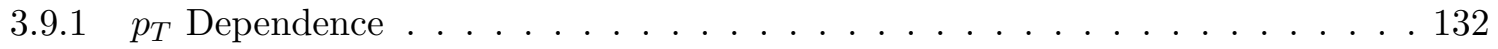

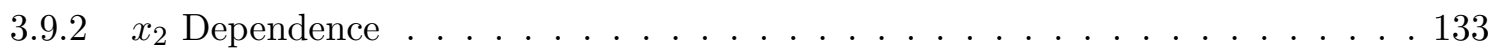

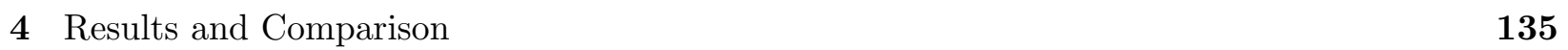

4.1 Results of Cross-section Ratios as a Function of $x_{F} \ldots \ldots \ldots$

4.2 Comparison $\ldots \ldots \ldots \ldots \ldots$

\begin{tabular}{|lll}
5 & Summary and Future Prospects & 144
\end{tabular}

\begin{tabular}{|lr}
\hline Bibliography & 147
\end{tabular}

\section{Appendix}

\begin{tabular}{|lc}
\hline A New Station-1 Drift Chamber & 153
\end{tabular}

A.1 The Wire Frame . . . . . . . . . . . . . . . . . . . . . . . . . . . . 154

A.1.1 High-voltage Distribution and Readout . . . . . . . . . . . . . . . 158

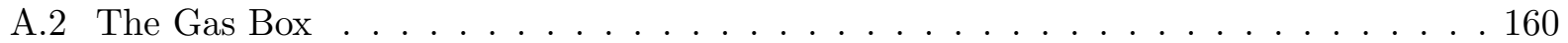




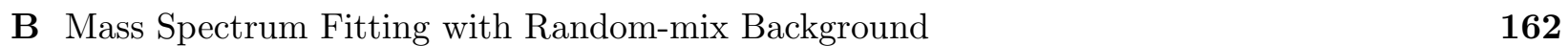

\begin{tabular}{lll}
\hline C Supplementary Plots and Tables for $R_{p A}\left(x_{F}\right)$ with $x_{2}>0.15$ & 172
\end{tabular}

C.1 Drell-Yan Yield Extraction and Average of Kinematics . . . . . . . . . . . . . . . 172

C.2 Rate-dependent Tracking Efficiency Correction $\ldots \ldots$. . . . . . . . . . . . 176

C.3 $R_{p A}\left(x_{F}\right)$ from Each Dataset $\ldots \ldots \ldots \ldots \ldots \ldots$. . . . . . . . . . . . . . . .

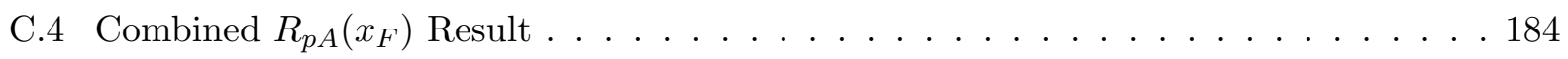




\section{Tables}

\section{Table}

1.1 Timeline of SeaQuest $\ldots \ldots \ldots \ldots \ldots \ldots \ldots \ldots$

2.1 Target configuration $\ldots \ldots \ldots \ldots \ldots \ldots \ldots \ldots$

2.2 Specifications of the hodoscope planes $\ldots \ldots \ldots \ldots$

2.3 Specifications of the wire chambers $\ldots \ldots \ldots \ldots \ldots$

2.4 Chamber combination of different run periods $\ldots \ldots \ldots \ldots$

2.5 Specifications of the proportional tube planes $\ldots \ldots \ldots \ldots \ldots$. . . . . . . 51

2.6 Definitions of Level-2 triggers $\ldots \ldots \ldots \ldots \ldots$

3.1 Description of Roadset $57,59,62$, and $67 \ldots \ldots \ldots$. . . . . . . . . 60

3.2 Summary of Roadset $57,59,62$, and 67 data $\ldots \ldots \ldots$. . . . . . . . . 61

3.3 Ranges of spills that are excluded from the analysis . . . . . . . . . . . . . . 61

3.4 Cuts on number of hits of detecors $\ldots \ldots \ldots \ldots$

3.5 Summary of cuts on spills $\ldots \ldots \ldots \ldots \ldots \ldots$

3.6 Summary of cuts on tracks $\ldots \ldots \ldots \ldots \ldots \ldots \ldots$

3.7 Summary of cuts on dimuons $\ldots \ldots \ldots \ldots \ldots \ldots \ldots$

3.8 Summary of cuts on target dimuons $\ldots \ldots \ldots \ldots \ldots$

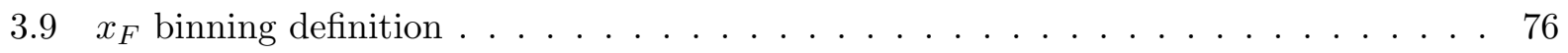

3.10 Target material properties $\ldots \ldots \ldots \ldots \ldots \ldots \ldots$

3.11 Number of live protons for each target in each data set $\ldots \ldots \ldots \ldots$ 
3.12 Estimation of the background number using the FPGA-3 events . . . . . . . . . . . 92

3.13 List of $F_{b k g}$ values $\ldots \ldots \ldots \ldots$. . . . . . . . . . . . . . . 99

3.14 Summary of background subtraction of Run-II data . . . . . . . . . . . . . . 100

3.15 Summary of background subtraction of Roadset 62 data . . . . . . . . . . . . . 101

3.16 Summary of background subtraction of Roadset 67 data . . . . . . . . . . . . . . 102

3.17 Average values of kinematic variables of Run-II data . . . . . . . . . . . . . . . . 104

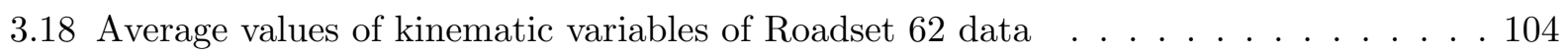

3.19 Average values of kinematic variables of Roadset 67 data $\ldots . . . . .105$

3.20 Parameters of rate-dependent $\epsilon_{R}$ fitting results of Run-II . . . . . . . . . . . . 114

3.21 Parameters of rate-dependent $\epsilon_{R}$ fitting results of Roadset $62 \ldots \ldots$

3.22 Parameters of rate-dependent $\epsilon_{R}$ fitting results of Roadset 67 . . . . . . . . 118

3.23 Average chamber intensity and $C^{c o r} \ldots \ldots \ldots \ldots \ldots \ldots$

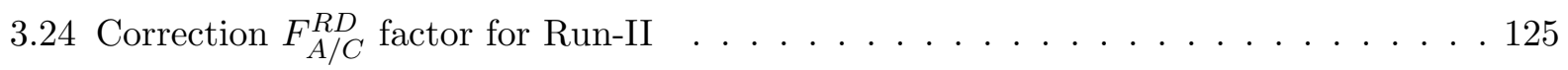

3.25 Correction factor $F_{A / C}^{R D}$ for Roadset $62 \ldots \ldots \ldots \ldots \ldots$

3.26 Correction $F_{A / C}^{R D}$ factor for Roadset $67 \ldots \ldots \ldots \ldots \ldots \ldots$

3.27 Estimated systematic error from remaining rate dependence . . . . . . . . . . . . 129

$4.1 \quad R_{p A}$ values and the corresponding errors for Run-II $\ldots \ldots$. . . . . . . . 136

$4.2 \quad R_{p A}$ values and the corresponding errors for Roadset $62 \ldots \ldots \ldots$

$4.3 \quad R_{p A}$ values and the corresponding errors for Roadset $67 \ldots \ldots$. . . . . . . 137

4.4 Combined $R_{p A}$ values and the corresponding errors $\ldots \ldots \ldots$. . . . . . . . . 139

A.1 Wire specifications of the new Station-1 chamber . . . . . . . . . . . . 156

C.1 Summary of background subtraction of Run-II data $\ldots \ldots \ldots$. . . . . . . . . 172

C.2 Summary of background subtraction of Roadset 62 data $\ldots \ldots$. . . . . . . . 173

C.3 Summary of background subtraction of Roadset 67 data $\ldots . . . . . .173$

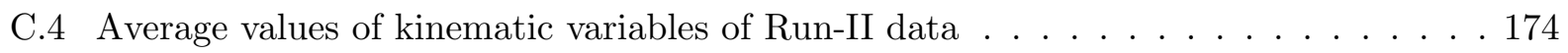




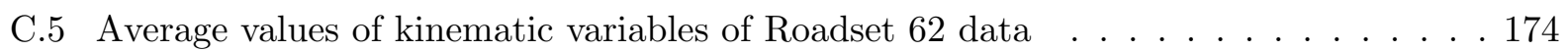

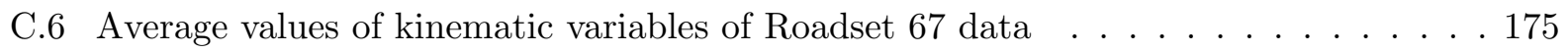

C.7 Average chamber intensity and $C^{c o r} \ldots \ldots \ldots \ldots \ldots$

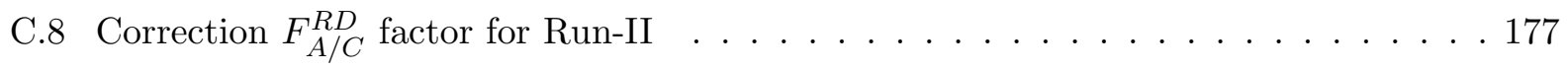

C.9 Correction $F_{A / C}^{R D}$ factor for Roadset $62 \ldots \ldots \ldots \ldots \ldots \ldots$

C.10 Correction $F_{A / C}^{R D}$ factor for Roadset $67 \ldots \ldots \ldots \ldots$

C.11 Estimated systematic error from remaining rate dependence . . . . . . . . . . . . . 181

C.12 $R_{p A}$ values and the corresponding errors for Run-II $\ldots \ldots$. . . . . . . . . . . 182

C.13 $R_{p A}$ values and the corresponding errors for Roadset $62 \ldots \ldots$. . . . . . 182

C.14 $R_{p A}$ values and the corresponding errors for Roadset $67 \ldots$. . . . . . . . . 183

C.15 Combined $R_{p A}$ values and the corresponding errors . . . . . . . . . . . . . 184 


\section{Figures}

\section{Figure}

1.1 Illustration of collisional and radiative energy $\operatorname{loss} \ldots \ldots \ldots \ldots$

1.2 Comparison of radiative and collisional energy loss for light and heavy quark partons as a function of the initial parton energy $\ldots \ldots \ldots \ldots \ldots$. . . . . . . . . 14

$1.3 \quad R_{A A}$ and $R_{p A}$ measured by PHENIX, CMS, and the ALICE experiments. . . . . . 15

$1.4 \quad$ Multiplicity ratio of HERMES $\pi^{+}$(full symbols) and $\pi^{-}$(open symbols) data and a illustration of a qurak propagation inside a nucleus in a DIS interaction $\ldots . . .17$

1.5 Cross section as a function of muon-pair mass $M_{\mu \mu} \ldots \ldots \ldots \ldots$

$1.6 \quad$ Illustration of the leading order Drell-Yan process $\ldots \ldots \ldots$. . . . . . . . . . . . . 19

1.7 The definitions of $\theta$ and $\phi$ in the Collins-Soper frame $\ldots \ldots \ldots$. . . . . . . . 20

$1.8 \quad$ Feynman diagrams of Drell-Yan to the $\operatorname{order} \mathcal{O}\left(\alpha_{s}\right) \ldots \ldots \ldots \ldots \ldots$

1.9 Parton distribution functions at NNLO from the CTEQ-TEA global analysis (CT14) 25

1.10 Energy loss process in Drell-Yan interaction $\ldots \ldots \ldots$. . . . . . . . . . 26

1.11 Measured per-nucleon cross section ratio $\sigma^{C(N)} / \sigma^{D}$ from DIS experiments . . . . . . 29

1.12 Nuclear modification factors in $\mathrm{Pb}$ nucleus $\ldots \ldots \ldots$. . . . . . . . . . . . . . 30

1.13 Per-nucleon cross-section ratio of $\mathrm{Fe} / \mathrm{Be}$ and W/Be from E866 with shadowing cor-

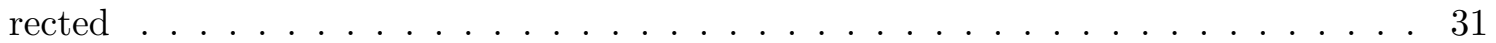

1.14 Per-nucleon cross-section ratio of W/D from E772 and W/Be from E866 with esti-

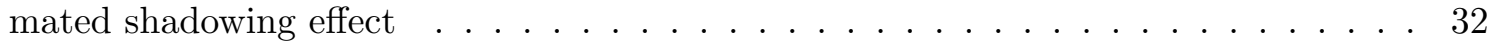

1.15 Theoretical prediction of per-nucleon cross section ratio for E906 . . . . . . . . . . . 34 
2.1 The E906/SeaQuest Spectrometer . . . . . . . . . . . . . . . . . . 35

2.2 The accelerator complex of Fermilab . . . . . . . . . . . . . . . . 37

2.3 The Čerenkov counter $\ldots \ldots \ldots \ldots$. . . . . . . . . . . . . . . . . . . . . . 39

2.4 Layout of the target table $\ldots \ldots \ldots \ldots \ldots$. . . . . . . . . . . . . . . . 41

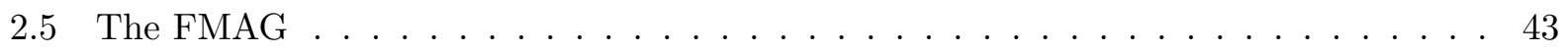

2.6 The KMAG $\ldots \ldots \ldots \ldots \ldots$

2.7 Schematic of the proportional tubes $\ldots \ldots \ldots \ldots \ldots$

2.8 Trigger acceptance versus mass for Run-II and Run-III FPGA-1 Roadsets . . . . . . 53

2.9 The workflow of the Main DAQ $\ldots \ldots \ldots \ldots \ldots \ldots$

2.10 Example of D3p hit distribution $\ldots \ldots \ldots \ldots \ldots$

3.1 Steps for reconstructing of a local hit triplet in a chamber . . . . . . . . . . . . . 64

3.2 Track projection to station 1 by sagitta ratio $\ldots \ldots \ldots \ldots$

3.3 Demonstration of Kalman filter implementation for track fitting . . . . . . . . . . . . 67

3.4 The workflow of Kalman-filter based track fitting $\ldots \ldots \ldots \ldots$

3.5 Treatment of a muon swimming through a single slice of FMAG $\ldots \ldots$. . . . . . . 68

3.6 Target-dump separation of dimuons $\ldots \ldots \ldots \ldots \ldots$

3.7 Kinematic distributions of the Monte Carlo events $\ldots \ldots \ldots$. . . . . . . . . 74

3.8 Comparison of kinematic variable distributions between like-sign random-mix and FPGA-3 data . . . . . . . . . . . . . . . . . . . . 83

3.9 Mass spectrum of tungsten target . . . . . . . . . . . . . . . . . 84

3.10 Comparison of distributions of kinematic variables of mass $<2.5 \mathrm{GeV} \quad \ldots \quad \ldots$. . . 85

3.11 Comparison of distributions of kinematic variables of mass $<2.5 \mathrm{GeV}$. . . . . 86

3.12 Comparison of distributions of kinematic variables of mass $<2.5 \mathrm{GeV}$. . . . . . 87

3.13 The intensity of $\mu^{-}$tracks versus the intensity of $\mu^{+}$tracks of random-mix data. . . 88

3.14 Comparison of mass distributions between the correct-intensity-mix data and the whole random mix data $\ldots \ldots \ldots \ldots$. . . . . . . . . . . . . . 89 
3.15 Mass distributions of Roadset 67 random-mix iron target events in nominal $x_{F}$ bins 93

3.16 Mass fitting in different $x_{F}$ bins of Run-II $\ldots \ldots \ldots$. . . . . . . . . . . . 96

$3.17 F_{b k g}$ of different $x_{F}$ bins of Run-II $\ldots \ldots \ldots \ldots \ldots$

3.18 Mass fitting in different $x_{F}$ bins of Roadset $62 \ldots \ldots \ldots$. . . . . . . . 97

$3.19 F_{b k g}$ of different $x_{F}$ bins of Roadset $62 \ldots \ldots \ldots \ldots$

3.20 Mass fitting in fine $x_{F}$ bins of Roadset $67 \ldots \ldots \ldots$. . . . . . . . . 98

$3.21 F_{b k g}$ of different $x_{F}$ bins of Roadset $67 \ldots \ldots \ldots \ldots$

3.22 Illustration of background subtraction in nominal $x_{F}$ bins of Run-II data $\ldots 100$

3.23 Illustration of background subtraction in nominal $x_{F}$ bins of Roadset 62 data $\ldots 101$

3.24 Illustration of background subtraction in nominal $x_{F}$ bins of Roadset 67 data $\ldots 102$

3.25 Comparison of kinematic variables of mass $>4.2 \mathrm{GeV}$ data after background sub-

traction from each data sets $\ldots \ldots \ldots \ldots$. . . . . . . . . . . . . . . . . . . . . . .

3.26 Comparison of normalized yield ratios $\ldots \ldots \ldots$. . . . . . . . . 106

3.27 Dimuon yield per trigger proton versus chamber intensity . . . . . . . . . . . . . 109

3.28 Rate dependence of $\epsilon_{R}$ with iron target data $\ldots \ldots \ldots$. . . . . . . . . 111

$3.29 \epsilon_{R}$ fitting in different $x_{F}$ bins of Run-II $\ldots \ldots \ldots \ldots$

$3.30 \epsilon_{R}$ fitting in different $x_{F}$ bins of Roadset $62 \ldots \ldots \ldots \ldots$

$3.31 \epsilon_{R}$ fitting in different $x_{F}$ bins of Roadset $67 \ldots \ldots \ldots \ldots$

3.32 D1 position of the tracks of the dimuons lost in reconstruction in different $x_{F}$ range 119

3.33 Normalized chamber intensity distributions in different mass ranges of Roadset 67 . 122

3.34 Chamber intensity of "none" target for different mass ranges of Roadset 67 . . . . 123

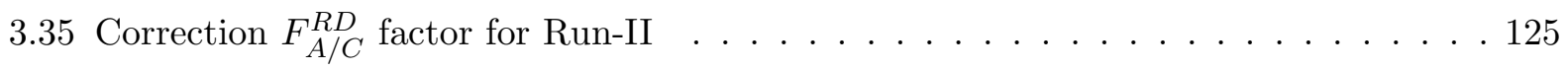

3.36 Correction factor $F_{A / C}^{R D}$ for Roadset $62 \ldots \ldots \ldots \ldots \ldots$

3.37 Correction factor $F_{A / C}^{R D}$ for Roadset $67 \ldots \ldots \ldots \ldots \ldots \ldots$

3.38 Corrected ratio of dimuon yield per trigger proton for each data set. . . . . . . . . 130

$3.39 p_{T}$ dependence of the Roadset-67 normalized yield ratios $\ldots \ldots \ldots$. . . . . . 133

3.40 Per-nucleon cross section ratios versus $x_{2} \ldots \ldots \ldots \ldots \ldots$ 
$4.1 \quad R_{p A}\left(x_{F}\right)$ with rate-dependence correction $F_{A / C}^{R D}$ applied. $\ldots \ldots \ldots \ldots$

4.2 The combined $R_{p A}\left(x_{F}\right)$ results. $\ldots \ldots \ldots \ldots \ldots \ldots \ldots \ldots \ldots$

4.3 The combined $R_{p A}\left(x_{F}\right)$ results including the one with $x_{1}$ cut. . . . . . . . . 140

$4.4 R_{p A}\left(x_{F}\right)$ with theoretic predictions $\ldots \ldots \ldots \ldots$. . . . . . . . . . . . . . .

4.5 The $A^{1 / 3}$ dependence of energy loss effect $\ldots \ldots \ldots$. . . . . . . . . . . . 143

A.1 Acceptance comparison between the old and new station-1 chambers . . . . . . . 153

A.2 Schematic of the layout of new Station-1 wire planes . . . . . . . . . . . . . . 154

A.3 Cell structure of the new Station-1 chamber . . . . . . . . . . . . . . . . . 155

A.4 The single-cell electric field and potential simulated by Garfield . . . . . . . . . . . 155

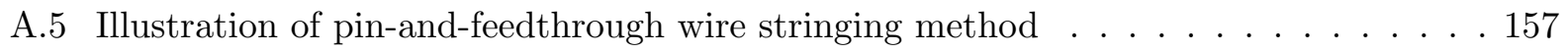

A.6 Signal-readout card and its connection to the Feedthrough-Boards attached on the

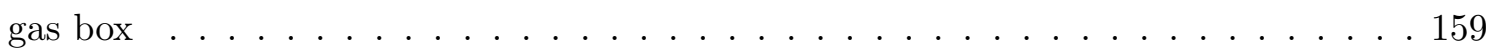

A.7 The high-voltage distribution board and its implementation . . . . . . . . . . . 160

A.8 The gas-box structure without the lid put on $\ldots \ldots \ldots \ldots 1$

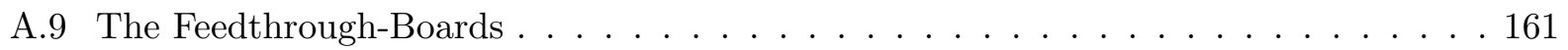

B.1 Mass spectrum fitting in nominal $x_{F}$ bins of Run-II iron data $\ldots . . . . . . .163$

B.2 Mass spectrum fitting in nominal $x_{F}$ bins of Run-II carbon data . . . . . . . . . . 164

B.3 Mass spectrum fitting in nominal $x_{F}$ bins of Run-II tungsten data $\ldots . . . . .165$

B.4 Mass spectrum fitting in nominal $x_{F}$ bins of Roadset-62 iron data $\ldots . . . . .166$

B.5 Mass spectrum fitting in nominal $x_{F}$ bins of Roadset-62 carbon data $\ldots . . . . .167$

B.6 $\quad$ Mass spectrum fitting in nominal $x_{F}$ bins of Roadset-62 tungsten data . . . . . . . 168

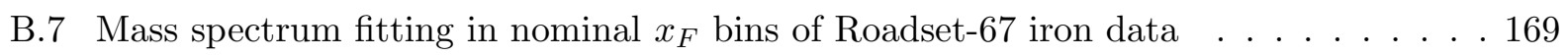

B.8 Mass spectrum fitting in nominal $x_{F}$ bins of Roadset-67 carbon data . . . . . . . . . 170

B.9 $\quad$ Mass spectrum fitting in nominal $x_{F}$ bins of Roadset-67 tungsten data . . . . . . . 171

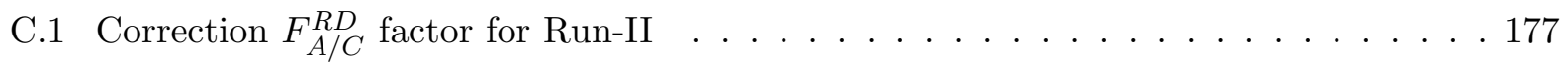


C.2 Correction $F_{A / C}^{R D}$ factor for Roadset $62 \ldots \ldots \ldots \ldots \ldots \ldots$

C.3 Correction $F_{A / C}^{R D}$ factor for Roadset $67 \ldots \ldots$. . . . . . . . . . 179

C.4 Corrected ratios of dimuon yield per trigger proton for each data set . . . . . . . . . 180

C.5 $\quad R_{p A}\left(x_{F}\right)$ with rate-dependence correction $F_{A / C}^{R D}$ applied. . . . . . . . . . . . . 181

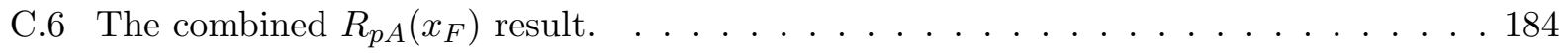




\section{Chapter 1}

\section{Introduction}

In this chapter, the motivation for quark energy loss studies with the E906/SeaQuest experiment is introduced by first giving a brief review of QCD and the characteristics of its perturbative treatment. The theoretical developments relative to parton energy loss, followed by some experimental observations, are then discussed. The Drell-Yan process and its ability to ideally measure the quark energy loss are subsequently explained in detail. Finally, the distinct advantages of performing the energy loss measurement at SeaQuest are presented.

\subsection{The Strong Interaction and Quantum Chromodynamics}

Being one of the four fundamental interactions currently known, the strong interaction is the interaction responsible for the formation of nucleons, and provides the binding between them to make various atomic nuclei. The development of the modern language for the strong interaction can be traced back to the early 1960s, as Gell-Mann and Zweig individually developed models [1, 2] in order to systematically explain the features of strongly interacting particles, which are called hadrons. As suggested in the models, hadrons are composed of more fundamental building blocks named as quarks; three quarks for baryons like the proton and neutron, and quark-antiquark pair for mesons like the pion. The experimental evidence supporting the existence of quarks then came in the late 1960s at the Stanford Linear Accelerator Center (SLAC). With deep inelastic scattering (DIS) experiments, a charged point-like substructure carrying spin of $1 / 2$ within the nucleon was revealed. Later in the early 1970s, in the effort of elucidating the interaction among quarks, Fritzsch, 
Gell-Mann, and Leutwyler proposed a gauge theory [3] based on the framework developed by Yang and Mills in 1954 [4], which gave birth to Quantum Chromodynamics (QCD).

As a non-Abelian $\mathrm{SU}(3)$ gauge theory, QCD is the most widely accepted theory for the description of the strong interaction and involves two fundamental degrees of freedom: quarks and gluons. The quarks and gluons are collectively referred to as partons, a term introduced by Feynman for the point-like constituent of hadrons, as in the parton model. Analogous to electric charge, the partons carry color charges and the strong interaction is mediated by the exchange of gluons, similar to the case of photon exchange in Quantum Electrodynamics (QED). The dynamics of the strong interaction are specified by the QCD Lagrangian which can be written as

$$
\mathcal{L}=\sum_{q=1}^{n_{f}} \bar{\psi}_{i}^{q} i \gamma^{\mu}\left(\partial_{\mu} \delta_{i j}-g_{s} t_{i j}^{a} A_{\mu}^{a}-m_{q} \delta_{i j}\right) \psi_{j}^{q}-\frac{1}{4} F_{\mu \nu}^{a} F^{a \mu \nu} .
$$

In this expression, the $\psi^{q}$ are the Dirac spinor quark fields of mass $m_{q}$ where $q$ represents the flavor of the quark, with the $i$ and $j$ being the color indeces that run from 1 to $N_{c}=3$. The $A_{\mu}^{a}$ are the four potential of the gluon fields with $a$ running from 1 to $N_{c}^{2}-1$. The $t_{i j}^{a}$ are the Gell-Mann matrices and $g_{s}=\sqrt{4 \pi \alpha_{s}}$ is the strong interaction coupling constant. The field strength tensor $F_{\mu \nu}^{a}$ is given as

$$
F_{\mu \nu}^{a}=\partial_{\mu} A_{\nu}^{a}-\partial_{\nu} A_{\mu}^{a}+g_{s} f^{a b c} A_{\mu}^{b} A_{\nu}^{c}
$$

where $f^{a b c}$ are the structure constants of the $\mathrm{SU}(3)$ group.

As a quantum field theory that needs to be renormalized, the strength of the coupling would depend on the renormalization scale $\mu$. This dependency is sometimes called the "running" of the coupling constant and is specified by the renormalization group equation. Solving the 1-loop renormalization group equation at leading order, one obtains the "alpha strong" $\alpha_{s}$ as

$$
\alpha_{s}\left(\mu^{2}\right)=\frac{g_{s}^{2}(\mu)}{4 \pi}=\frac{4 \pi}{\left(\frac{11 N_{c}}{3}-\frac{2 n_{f}}{3}\right) \ln \left(\frac{\mu^{2}}{\Lambda_{Q C D}^{2}}\right)},
$$

where the $\Lambda_{Q C D}$ is the constant QCD scale of order $200 \mathrm{MeV}$, indicative of the scale at which the coupling of $\mathrm{QCD}$ diverges. The renormalization scale $\mu$ is an unphysical scale usually chosen to be close to the relevant hard scale, $Q$, such as the momentum transfer, of a specific process, 
and $\alpha_{s}\left(\mu^{2} \simeq Q^{2}\right)$ represents the effective strong interaction strength in that process. Evidently from Eq. 1.3, the coupling strength shows a logarithmic decrease with increasing energy scale and correspondingly decreasing distance scale. The fact that $\alpha_{s}$ becomes small in the high-energy, shortdistance regime is the distinctive and crucial feature of QCD discovered by Politzer [5], Gross, and Wilczek[6], and is referred to as asymptotic freedom.

Asymptotic freedom grants us the ability to perform perturbative QCD (pQCD) treatments; by carrying out a perturbative expansion with small parameters of $\alpha_{s}$, one can reliably evaluate physical quantities to the desired order. However, pQCD calculations are performed in terms of quark and gluon fields which are actually not the physically measurable particles due to confinement, a QCD property on the other end of the energy spectrum. As indicated by confinement, the asymptotic states of strong interaction processes would not be quarks and gluons but the composite hadrons. This suggests the inevitable involvement of non-perturbative, long-distance behavior of QCD in the calculations and asymptotic freedom alone is insufficient to make pQCD a useful and predictive tool in some regime.

The bridge between experimental measurements and the pQCD calculation is served by factorization theorems [7, 8], which demonstrate the ability of systematic separation (factorization) of the hard, short-distance physics associated with the large momentum scale $Q$ from the remaining long-distance, soft part which is of a non-perturbative nature. The short-distance portion can be calculated with the perturbation theory on the partonic level, while the long-distance part needs (at the current stage) to be determined by experiments with phenomenological approaches. The feasibility of factorization is rooted in the absence of interference between the dynamics at the short-distance and long-distance scale, which can be naïvely justified by asymptotic freedom and features of quantum mechanics and special relativity. Nevertheless, rigorous proof of factorization is highly nontrivial and depends on various issues like the specific hard process to be dealt with and the order in the perturbative expansion considered. Thus sometimes factorization is simply assumed and only tested by experiment.

Although the factorized non-perturbative part could not be fully determined from QCD, 
it can be accessed via well-defined operator expressions which then lead to functions of different physical interpretations, such as parton distribution functions (PDFs) or fragmentation functions (FFs). The PDFs are correlated to the long-distance physics related to the initial states and can be understood as the probability densities to find partons in the hadrons, while the FF, correlated with final states, can be interpreted as the probability densities of producing hadrons from partons. These functions carry the following important properties:

- Universality: The PDFs and FFs are universal regardless of the specific hard partoniclevel interaction involved. For example, the PDFs extracted from DIS should be identical to the one from the Drell-Yan interaction, the interaction that will be explained in more detail later.

- Scale dependence: Similar to the renormalization technique that results in the running of $\alpha_{s}$ as mentioned before, the PDFs and FFs satisfy renormalization group equations that gives their scale dependence. The evolution with scale of the PDFs and FFs is generally referred to as QCD evolution and can be calculated by means of the Dokshitzer-GribovLipatov-Altarelli-Parisi (DGLAP) equation [9-11].

Thus, with the features mentioned above, one can, for example, measure the PDFs in a limited energy range, follow the evolution equations and extrapolate the PDFs to arbitrarily high energies, and then use the PDFs acquired as input to make predictions for processes that are not necessarily the same as the one with which the PDFs are determined, even for ones including new physics. The various successful verifications of these factorization-theorem-based features and their application over a wide range of energy scale have been regarded as a remarkable success of QCD.

\subsection{Parton Energy Loss}

Having introduced some important aspects and treatments of pQCD, we now turn to the primary focus of this thesis, parton energy loss in the presence of nuclear matter. There are studies of the energy loss effect at the hadronic level, like the one discussed in [12], but they are beyond 
the scope of this thesis and will not be discussed in detail.

For electrically charged particles, in their passage through matter, they lose energy by electromagnetic interactions like the ionization of the medium, radiation, and even $e^{+} e^{-}$pair production. Similarly, the energetic partons can interact with colored nuclear matter via the predominating QCD processes which then result in the loss of energy. The energy loss of partons is a fundamental phenomenon within QCD that draws significant theoretical and experimental interest, but a solid understanding of it has not yet been reached. Being an interesting topic in its own right, the quest for a better knowledge of the parton energy loss also comes from its applications. For example, due to the fact that the energy loss mechanism of partons is closely correlated to the nature of the medium that the partons propagate through, the energy loss can serve as a probe sensitive to the properties of a nuclear matter, just like X-ray tomography in the electromagnetic case.

In general, nuclear-matter-induced parton energy loss can be divided into two categories: collisonal or so-called elastic energy loss, and radiative or inelastic energy loss. These two energy loss processes are illustrated in Fig. 1.1 and briefly described as follows.

- Collisional energy loss: Collisional energy loss can be depicted as a $2 \rightarrow 2$ scattering of the incoming primary parton with the medium through a gluon exchange. Conceptually, the scattering induces recoil effects in the medium, and gives rise to an energy loss of the incoming parton in a way that the virtuality (off-shellness) of the parton is not drastically changed after the scattering.

- Radiative energy loss: In a radiative energy loss process, there can be multiple scattering between the primary parton and the medium, with which the virtuality of the parton is increased. Eventually the radiation of a gluon from the parton is induced so that some part of the part of the energy is carried by the radiated gluon and the virtuality of the incoming parton is reduced.

Compared to the QED case, the QCD version of energy loss is complicated by factors like medium modeling, which is correlated to the long-distance behavior of QCD, and the calculation of 


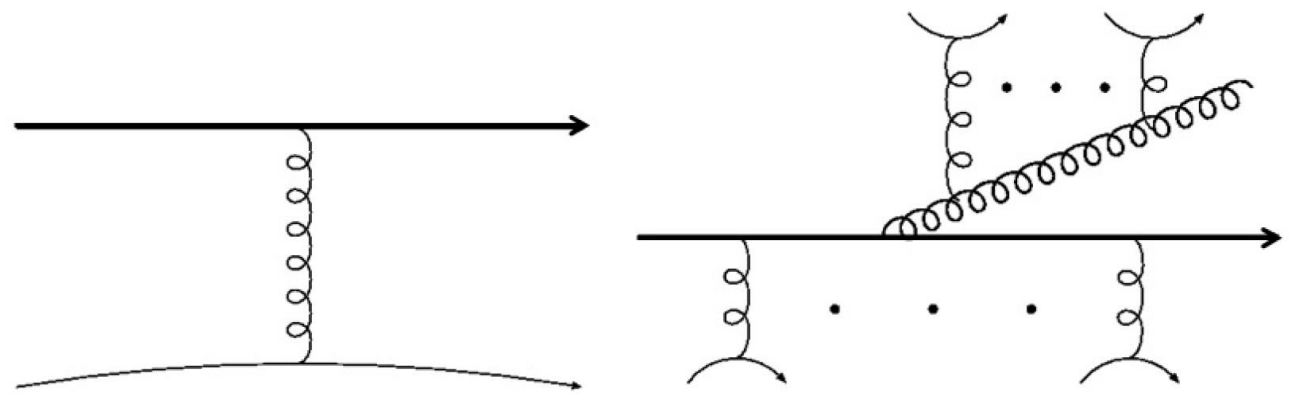

Figure 1.1: Illustration of collisional (left) and radiative (right) energy loss of a parton, represented as the thick arrow, traveling through a nuclear matter [13].

scattering amplitudes and the interference between them. The theoretical formulation for radiative and collisional energy loss will be introduced separately in the upcoming sections. Rather than covering all the theoretical treatments and going through the technical details of this large and growing field, only the studies within current focus are pointed out. The emphasis will be placed on the essential elements, features, assumptions, and limitations of different theoretical frameworks in order to give an overall picture of the theoretical developments of parton energy loss.

\subsection{Radiative Energy Loss}

At the present time, radiative energy loss is considered to be the dominating factor in exploring the energy loss effect and has attracted more theoretical effort, compared to collisional energy loss. The core of the theoretical treatment of radiative energy loss is the evaluation of the medium-induced single-gluon-emission kernel, such as the differential gluon-radiation cross section, where the cross section stands for the measure of how likely a process would occur in units of area. Within the evaluation, the medium modeling and the interaction between the medium, the primary parton, and the radiated gluon are handled. Single-gluon-emission kernels are then utilized to form the basis for the description of possible multiple gluon emission.

The early investigation of energy loss of a charged particle via photon radiation carried out by Landau, Pomeranchuck, and Migdal [14, 15] plays a crucial role in initiating the theoretical 
interest in formulating the QCD analogue of this radiative energy loss. The QCD extension [16] of a QED effect that was first brought up by Landau et al. is important to the general radiative energy loss study. Similar to QED formulation introduced by Landau et al., the formation time $\tau_{f}$ for a gluon radiation can be characterized by the time it takes for the transverse separation between the gluon and the radiating parton to be of the order of gluon transverse wavelength. $\tau_{f}$ is therefore expressed as $\tau_{f} \simeq 2 w / k_{T}^{2}$, where $w$ and $k_{T}$ are the energy and transverse momentum of the radiated gluon. In the situation of small-angle emission in which the radiated gluon would propagate through a path similar to the leading parton, the formation time can be larger than the time (specified by the mean free path) between the multiple scatterings experienced by the propagating parton. The successive scatterings therefore cannot be regarded as independent and would lead to a deconstructive interference that result in the suppression of gluon radiation, as compared to the Bethe-Heitler limit where the scatterings are treated incoherently. This suppression of radiation is referred to as the Landau-Pomeranchuck-Migidal (LPM) effect.

Another general issue that needs to be considered in the medium-induced radiative energy loss study is virtuality and correlated vacuum splitting. For a parton carrying high virtuality, such as the hard-collision-produced one with high transverse momentum, it can go through vacuum splitting processes and have gluons emitted to reduce its off-shellness even without a medium. In the case of gluon radiation with the presence of a medium, ambiguities can arise in the treatment of incorporating the vacuum radiation and the interference between the vacuum and medium-induced splitting.

In the following, a few commonly-employed theoretical approaches for radiative energy loss calculations are going to be discussed. The schemes for the single gluon emission will be introduced first, where a general description of various schemes is given, followed by some comparison of the medium modeling and kinematic assumptions. The ways of generating multi-gluon emission are covered in the next subsection. For convenience of discussion, the energy of the primary parton will be represented as $E$, the transverse momentum exchanged with medium through scattering as $q_{\perp}$, the energy and the transverse momentum of radiated gluons as $w$ and $k_{T}$, and the extent of 
the medium as $L$.

\section{BDMPS-Z and ASW-MS Formalisms}

In the BDMPS-Z formalism of gluon radiation developed by Baier, Dokshitzer, Mueller, Peigné, and Schiff (BDMPS) [16, 17] and independently by Zakharov [18, 19], the medium structure is modeled as a set of static scattering centers with color screened Yukawa potential. This type of medium modeling was introduced by Gyulassy and Wang [20, 21] and is often referred to as the Gyulassy-Wang (GW) model. The single gluon radiation kernel is formulated in terms of a path integral in which the multiple scattering between the incoming parton and the radiated gluon, and the scattering centers are resummed. The so-called soft-gluon-emission approximation, which takes the $w / E \ll 1$ limit, is used in the original calculation in BDMPS, and was later expanded to include a finite value of $w / E[22]$. The interference between vacuum and medium-induced gluon radiation with the path integral formulation was taken into accounted by Wiedemann [23].

The BDMPS-Z scheme was incorporated in the work of Armesto, Salgado, Wiedemann (ASW) [23 26], in which the analytic expressions and numerical accessible forms are given. In most of the discussions of the BDMPS-Z formalism, the interaction between the projectile (the primary parton or radiated gluon) and the medium is taken to be in the multiple soft scattering regime. Within this limit, the Brownian motion in transverse momentum of the projectile dominates and the path integral in the ASW formulation can be carried out via the saddle-point approximation. This implementation of ASW is usually denoted as ASW-MS, in which the medium effect in the coherent limit can be characterized by a single parameter, the transport coefficient $\hat{q}$, defined as the average transverse momentum squared gained by the projectile per unit distance $\lambda$ traveled through the medium

$$
\hat{q}=\frac{q_{\perp}^{2}}{\lambda}
$$

\section{GLV and ASW-SH Formalisms}

Similar to the BDMPS-Z formalism, modeling the medium with Debye screened static scattering centers is adopted in the opacity expansion approach, which was pioneered by Gyulassy, 
Levai, and Vitev (GLV) [27 29] and independently by Wiedemann [23]. However, rather than taking the multiple-soft-scattering limit, they formulated the gluon radiation process on the basis of a relatively thin medium and only a few and rather hard scatterings are involved. The single gluon radiation kernel in the GLV approach is then evaluated by the systematic expansion in orders of opacity, $L / \lambda$, where $\lambda$ is the mean free path of the parton and opacity can be understood as the mean number of collisions in the medium.

In the opacity expansion of order $N$, the $N=0$ term is medium independent and represents the vacuum-radiation contribution. The leading medium dependence is determined by the $N=1$ term and the interference of vacuum and in-medium radiation is included for an off-shell primary parton. The $N>1$ higher-order contributions renders corrections from coherence effects and are suggested to be small [29]. Thus, most of the calculations include terms only up to $N=1$, which is sometimes called as the single hard scattering approximation. The parameters for specifying

the medium property in this approach are the Debye screen mass, $\mu_{D}$, which characterizes the range of the medium potential as $1 / \mu_{D}$, and the scattering-center density (or the mean free path $\lambda$, equivalently).

The opacity-expansion approach has several implementations in which different kinematic approximations are taken, like the single-hard-scattering limit of ASW formalism (ASW-SH) or the DGLV [30, 31]. An extension of the DGLV approach to dynamical scattering centers is explored in the work by Djordjevic and Heinz [32].

\section{AMY Formalism}

In the formalism introduced by Arnold, Moore, Yaffe (AMY) [33 35], the gluon radiation description is developed under the framework of effective thermal field theory, in which temperature is a natural representation of the relevant energy scale. The medium is modeled as a collection of high-temperature, weakly interacting quasi-particles of quarks and gluons in thermal equilibrium. With Hard Thermal Loop (HTL) perturbation theory, the gluon radiation spectrum induced by multiple soft scatterings with the medium can be calculated with the gluon self-energy in the soft thermal background. The virtuality of the incoming parton in the AMY approach is assumed to be 
nearly on-shell, so the vacuum radiation is not taken into account and only the scattering induced emission is considered. Rather than static scattering centers adopted in the previously introduced formalisms, the thermal medium in AMY consists of moving dynamic scatterers. The applicability of the AMY medium modeling, however, is in principle limited to the very high temperature regime due to the required perturbative nature.

In the original AMY formulation, since the calculation is performed in momentum space, infinite medium length is implicitly assumed. The effect of a finite size medium was later explored by Caron-Hout and Gale [36]. The medium modeling parameters are usually chosen as the temperature $T$ or the strong coupling constant $\alpha_{s}$.

\section{HT Formalism}

In the higher-twist (HT) formalism pioneered by Guo and Wang [37, 38], rather than making a specific definition of the medium, they proceeded in a way of more field-theory rigor. The energy loss derivation in the HT approach is based on the gluon emission of a quark produced in deeply inelastic electron-nucleus scattering, in which the description of the nucleon structure is expressed in a series of terms of matrix elements, which contain the products of local operators and coefficient functions. These terms are suppressed by powers of the hard scale $Q^{2}$ of the process and the magnitude of suppression depends on the twist of local operator, which is defined as the mass dimension minus the spin. The contribution from higher-twist (HT) terms is therefore smaller compared to the one from leading-twist (LT) ones in the high $Q^{2}$ limit.

To address the nuclear medium effect, the scattering with medium is considered as coming from another nucleon in the nucleus and treated as HT corrections to the LT results, where the gluon-emitting primary quark is taken to be highly off-shell. Therefore, in HT formalism, the medium property is encoded in the HT matrix elements, and the effect of vacuum radiation is considered. Initially, the HT formalism derives only corrections of single scattering, and was subsequently extended to include multiple-scattering contributions by Majumder [39]. The medium

effect in HT formalism is parameterized as the two-gluon field strength correlator, which can be translated to the transport coefficient $\hat{q}$ as defined in Eq. 1.4. 


\section{Discussion of Energy Loss Formalisms}

For all the formalisms previously discussed, the radiative energy loss is induced by transverse momentum exchange with the medium and three kinematic assumptions are made in the calculations.

- Both of the energies of the primary parton and the emitted gluon are much larger than the transverse momentum transferred between the medium, that is, $E, w \gg q_{\perp}$.

- The energy of the gluon is much larger than its transverse momentum, $w \gg k_{T}$, which corresponds to a small-angle radiation.

- The scattering between the hard parton and the medium is localized.

Additionally, it is often assumed that primary parton energy is much greater than the gluon energy, $E \gg w$. This so-called soft radiation approximation is not adopted in the AMY formalism. These approximations are introduced for the sake of simplifying the calculation involved, but can bring problems to phenomenological approaches that try to make applications in an extended phase space. In practice, this issue is patched up by making specific kinematic cutoffs.

As can be seen, each formalism has its own parameterization for characterizing medium properties. These medium-modeling parameters, however, can be related with each other by approaches, such as the one discussed in [40] that uses the HTL theory as a base. Lately, it is quite general to have the medium parameters translated into an effective transport coefficient, $\hat{q}$. Despite the many differences between various formalisms, in the detailed comparison made by [40], it has been suggested that the quantitative divergence between different models may be largely due to those specific approximations adopted in their calculations.

\subsubsection{Multiple Gluon Emission}

Going beyond emitting a single gluon, multiple gluon emission could be induced by the medium. Currently, the phenomenological approaches for multi-gluon emission are essentially to 
employ the single-gluon-emission kernel repeatedly as required. Apparently, the interference between different emissions could have non-negligible effect and should be taken into account in a thorough calculation. So far, studies about the interference between two emitters by considering an antenna of massive quark-antiquark pair have been attempted [41, 42, but the generalization to multiple emissions is not yet achieved.

One commonly adopted method for multi-gluon emission is assuming the number of gluon emissions follows a Poisson probabilistic description with the mean number derived by the integral of single gluon emission spectrum. This procedure, generally referred to as the Poisson ansatz, is usually applied in phenomenological calculations based on ASW or GLV formalisms for evaluating the probability distribution of energy loss. There is, however, one concern with this rather straightforward approach. Since the momentum loss of the primary parton due to gluon radiation is not dynamically updated after each emission, the derived energy loss spectrum can have values greater than the incoming parton energy and thus violates the energy conservation. Nevertheless, in general cases the overall probability for this is small and regarded as the probability for the parton to lose all of its energy.

For the AMY and HT formalisms, the emission of multiple gluons is calculated via different evolution procedures. In AMY's case, multi-gluon radiation is implicitly considered by evaluating the time evolution of the parton momentum distribution, $P(E, t)$, according to the rate equations [43] that have the generic form:

$$
\frac{d P(E, t)}{d t}=\int d w\left[P(E+w, t) \frac{d \Gamma(E+w, w)}{d w}-P(E, t) \frac{d \Gamma(E, w)}{d w}\right]
$$

In this expression, the $d \Gamma(E, w) / d w$ is the transition rate for a parton of energy $E$ to lose energy $w$. On the other hand, the medium-modified DGLAP evolution equation is employed for the HT formalism. In the treatments using evolution equations, the projectile partons are kept track of along their propagation through the medium, and the radiation probability changes correspondingly to the decrease of parton energy. Compared to the Poisson ansatz, the evolution of energy loss is better controlled and the conservation of energy is held within these procedures. There are other 
existing issues during propagation, such as the change of remaining path length and the evolution of medium property. However, combining all possible effects with a finite radiation formation time $\tau_{f}$ is a fairly challenging problem and has not yet been well addressed.

\section{$1.4 \quad$ Collisional Energy Loss}

As early as 1982, the collisional energy of partons was investigated by Bjorken [44. Although radiative energy loss has generally been regarded as the main contribution of energy loss, recent studies suggests that the collisional process also plays a considerable role, especially in explaining the experimental observation from heavy quark-flavor. For the quarks of large mass, the gluon emission at small angle is strongly suppressed due to the so-called dead-cone effect, which can be understood as a cut-off in kinematic phase space.

There have been theoretic attempts to incorporate the elastic energy loss into the energyloss formalisms previously mentioned in the section on radiative energy loss, and many currently developed collisional energy-loss calculations are formulated in the framework of effective thermal field theory [43]. Within the AMY formalism, since the medium is modeled as composed of dynamic scatterers, the recoiling of the medium partons is naturally accounted for and the energy loss induced by collisions can be evaluated on the same footing as the one from radiative processes. In the HT formalism, the effect of introducing the collisional energy loss can be characterized by the per-unitlength longitudinal momentum transfer in scattering (also called the drag) and the variance of this momentum transfer per unit length. For the original BDMPS-Z, ASW, and GLV formalisms which employ static scattering centers, elastic collisions with the medium are forbidden and the collisional

energy loss is thus independently imposed in phenomenological approaches. Nevertheless, this issue has been explored in the DGLV extension of the GLV formalism that adopts dynamic scatterers, with which both energy loss mechanisms can be formulated. Fig. 1.2 shows a comparison between the two energy-loss processes that are derived based on AMY and HT formalisms. As can be seen, the radiative energy loss dominates in the high-energy regime, while the collisional loss provides sizable contribution at lower energies. 

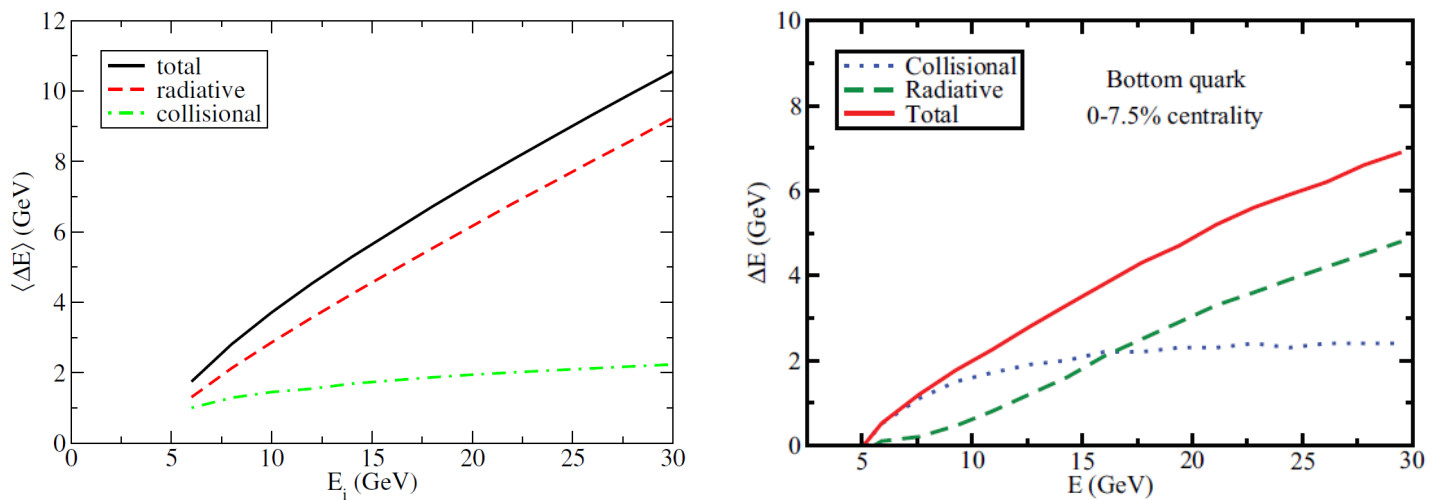

Figure 1.2: Comparison of radiative and collisional energy loss for light and heavy quark partons as a function of the initial parton energy. The left plot shows the average energy loss of a light parton evaluated based on the AMY formalism [43, and the right plot gives the energy loss of a bottom quark calculated within the HT formalism [45].

\subsection{Experimental Measurements}

The measurement of parton energy loss is not as simple as its electromagnetic counterpart. Since the color carrying partons cannot be directly measured due to confinement, the energy loss of partons can only be determined indirectly through the modification of experimental measurables that are modified by the energy loss effect. An exemplary phenomenon that has been extensively investigated for the energy loss study in high-energy particle collisions is called "jet quenching".

A jet is a collection of approximately collimated particles produced by the hadronization of an energetic primary parton. In a general definition, jet quenching refers to the colored-medium induced modification of the evolution of this primary parton. One of the main observables of jet quenching is the hadron production spectrum in the region of high transverse momentum $p_{T}$. Using the corresponding high- $p_{T}$ primary partons produced in the early-stage hard scatterings as probes, the information about the interaction with the medium propagated through by the partons is encoded in the behavior of the particles detected. Experimentally, a way to quantify the jet quenching at high- $p_{T}$ by colliding two nuclei $(A+A)$ is to measure the nuclear modification factor 

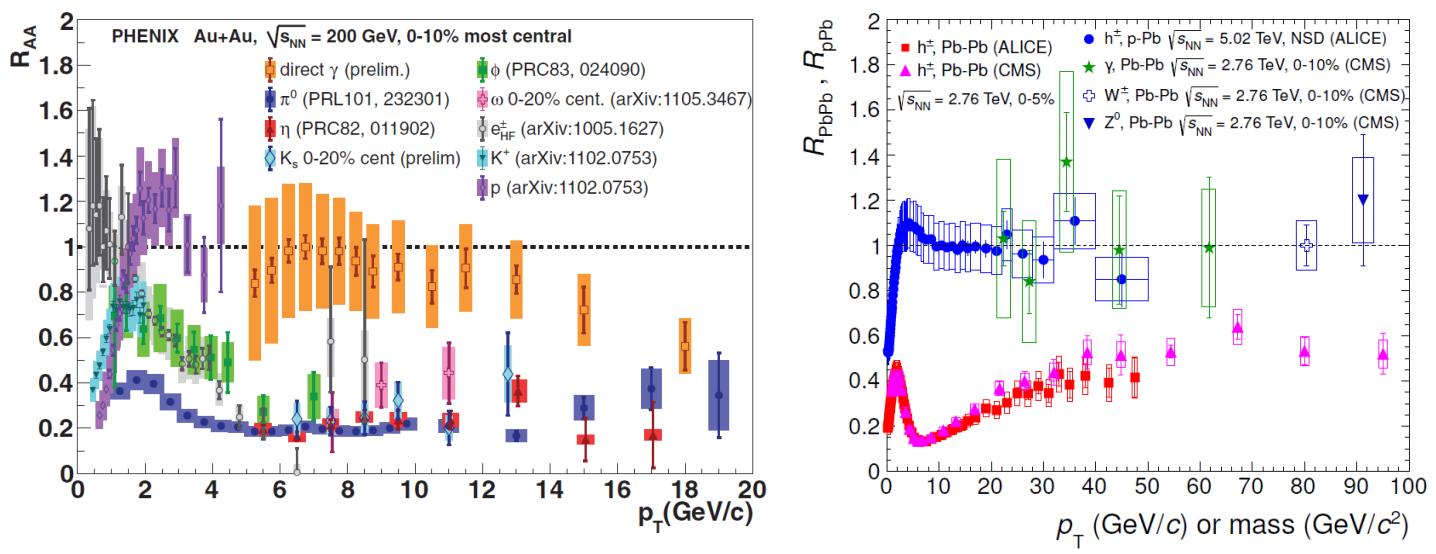

Figure 1.3: $R_{A A}$ and $R_{p A}$ measured by PHENIX, CMS, and the ALICE experiments. Left: $R_{A A}$ of various identified particles in central $\mathrm{Au}+\mathrm{Au}$ collisions at PHENIX [46]. Right: $R_{A A}$ in $\mathrm{Pb}+\mathrm{Pb}$ central collisions measured at CMS and ALICE, and $R_{p A}$ of $\mathrm{p}-\mathrm{Pb}$ collisions measured at midrapidity by ALICE. This plot is from [47].

$R_{A A}$, which is generally defined as

$$
R_{A A}\left(p_{T}\right)=\frac{1}{N_{\text {coll }}} \frac{d N^{A A} / d p_{T}}{d N^{p p} / d p_{T}}
$$

where $N_{\text {coll }}$ is the mean number of binary nucleon-nucleon collisions, and $N^{A A}$ and $N^{p p}$ are the single-inclusive hadron yields in nucleus-nucleus and proton-proton collisions, respectively. Likewise, $R_{p A}$ can be defined with one of the colliding particles being a proton. Shown in Fig. 1.3 is an example of $R_{A A}$ and $R_{p A}$ measurements from experiments using the Relativistic Heavy-Ion Collider (RHIC) at Brookhaven National Laboratory (BNL) and the Large Hadron Collider (LHC) at European Organization for Nuclear Research (CERN). In the absence of medium effects, the $R_{A A}$ at high $p_{T}$ should exhibit binary-collision scaling and is expected to be consistent with unity, which is what can be seen for particles not sensitive to QCD dynamics (such as $\gamma$, W, and Z). However, a clear suppression in hadron production yields is observed in A-A collisions, but not in p-A ones. These results indicate the strong suppression is a consequence of final-state effects, and has been regarded as one of the key features of energy loss in a hot and dense QCD matter created in heavy-ion collisions, the quark-gluon plasma (QGP).

In the phenomenological analyses of jet quenching, since there is not yet a solid theoretical 
verification that the cross section factorizes, factorization is assumed and a general cross-section form is adopted:

$$
d \sigma_{A B \rightarrow h X} \approx \sum_{a b p p^{\prime} d} f_{a / A} \otimes f_{b / B} \otimes d \sigma_{a b \rightarrow j d} \otimes P_{j \rightarrow j^{\prime}} \otimes D_{h / j^{\prime}}
$$

where $d \sigma_{A B \rightarrow h X}$ is the cross section of producing hadron $h$ and anything else $X$ by colliding hadrons $A$ and $B . f_{a / A}$ and $f_{b / B}$ are the PDFs for parton $a$ and $b$, respectively. $d \sigma_{a b \rightarrow j d}$ denotes the parton-level cross section for the process producing a parton $j . P_{j \rightarrow j^{\prime}}$ describes the effect of the colored medium and the resulting evolution of parton $j$ to $j^{\prime}$, and finally $D_{h / j^{\prime}}$ represents the FF for the parton $j^{\prime}$ to fragment into hadron $h$. The $P_{j \rightarrow j^{\prime}}$ piece in the expression above is where the final-state energy loss effect is introduced and is often combined with the vacuum FF to define the medium-modified FF for convenience. So far, the factorization assumption works consistently in phenomenological studies and the energy loss, being sensitive to medium properties, has been an important tool for understanding the novel characteristics of QGP. On the other hand, other than the parton energy loss, the phenomenological description of jet quenching is convoluted with a number of factors together with their uncertainties, such as the initial-state jet production spectrum, the cold-nuclear-matter effects, and the hadronization of partons. With the complexity involved, the energy loss extraction in heavy-ion collisions is quite uncertain at the current stage.

Other than the various attempts to determine the energy loss in hot QCD matter, there also have been efforts trying to measure the energy loss in ordinary cold nuclear matter. A precise knowledge of the energy loss in cold nuclear matter can not only be used to compare with the predictions given by different theoretical approaches, but also provides valuable information for differentiating the behavior of QGP from the ordinary nuclear matter. For the data collected by DIS experiments, such as HERMES [48] and CLAS [49], the cold-nuclear-matter energy loss is typically studied by the hadronic multiplicity ratio $R_{M}^{h}$, which represents the normalized modification in production yield of hadrons of type $h$ with a nuclear target compared to that from a deuterium target. The left panel of Fig. 1.4 shows the HERMES results of $R_{M}^{\pi}$ as a function of $z$, where $z$ is the energy fraction of the virtual photon transferred to the pion. The observed attenuation of $R_{M}^{\pi}$ 
can be interpreted as the effect of final-state parton energy loss and treated in a way similar to the heavy-ion collision case. The calculations employing the ASW-MS approach [50] is also shown in Fig 1.4 as the blue curves.

However, the suppression of $R_{M}^{h}$ could be affected by the hadron formation (hadronization) process. As illustrated in the right panel of Fig 1.4, some existing models suggest that the quark struck by the virtual photon propagates over some distance in the nuclear matter, lose its energy, and then start to form a "pre-hadron" state, in which the partons carry no net color and have quantum numbers identical to a hadron that this state is going to turn into. Since the pre-hadron is colorless, it interacts with the medium less than a single colored parton. This hadronization process is not well understood yet but if it starts inside the medium, the interaction of the prehadron with the medium can contribute to the hadron suppression observed [50 52] and thus obscure the partonenergy-loss extraction. On the other hand, this ambiguity is absent in the Drell-Yan process, which has been regarded as the ideal tool for measuring parton energy loss in cold nuclear matter [53, 54. In the following, the Drell-Yan process and its unique role in energy-loss measurements will be discussed in detail, and the advantages of conducting such measurements in E906/SeaQuest experiment will be explained.
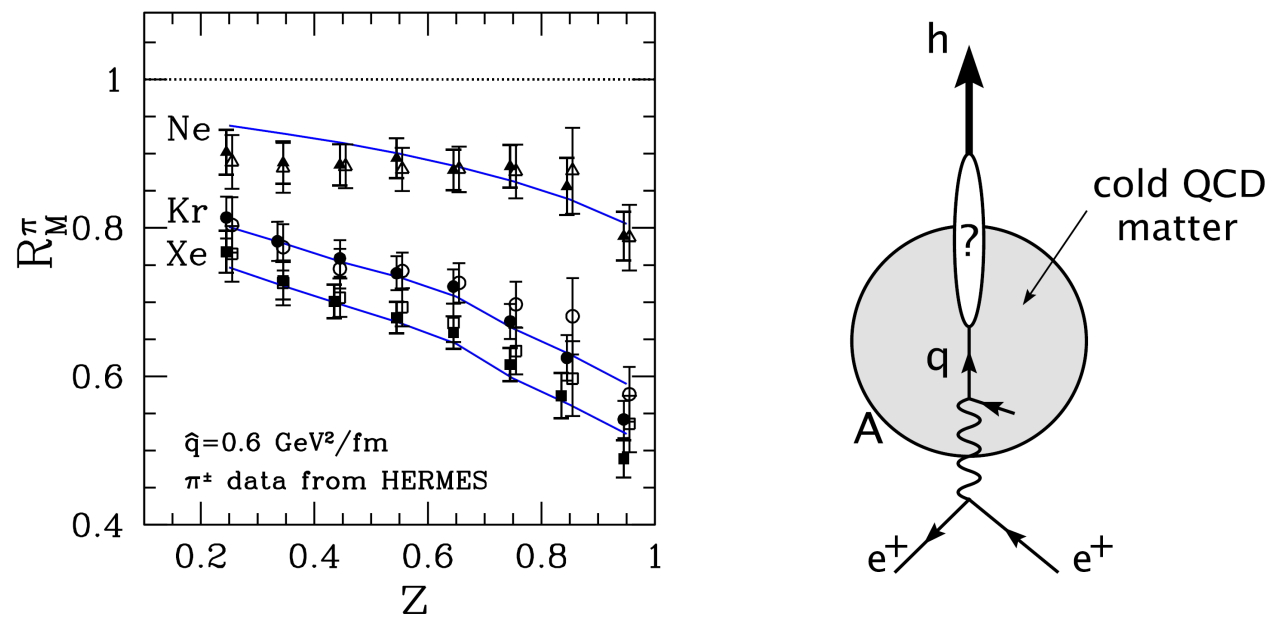

Figure 1.4: Left: Multiplicity ratio of HERMES $\pi^{+}$(full symbols) and $\pi^{-}$(open symbols) data compared to the ASW-MS energy loss calculations [50, 55]. Right: An illustration of a qurak propagation inside a nucleus in a DIS interation. [55] 


\subsection{The Drell-Yan Interaction}

The Drell-Yan process was first proposed by Sidney Drell and Tung-Mao Yan in 1970 [56] in order to provide further insight into the production of high-mass lepton pairs (dileptons) in inelastic hadron-hadron collisions on the basis of parton model. The experimental interest in lepton pair production began with the study of muon pairs as reported in Ref. [57, [58]. By using the proton beam from the alternating-gradient synchrotron (AGS) at BNL, the reaction

$$
p+U \rightarrow \mu^{+}+\mu^{-}+\text {anything }
$$

was investigated. The effective mass spectrum of the muon pairs $M_{\mu \mu}$ was measured in the range of $1<M_{\mu \mu}<6.7 \mathrm{GeV}$. Figure 1.5 shows a shoulder-like structure near $3 \mathrm{GeV}$ sitting on a rapidly falling, smooth background. The bump around $3 \mathrm{GeV}$ was later identified as the $J / \psi$ resonance, the bound state of a charm quark and a charm antiquark. The rest of the continuous mass distribution, on the other hand, is due to the Drell-Yan process.

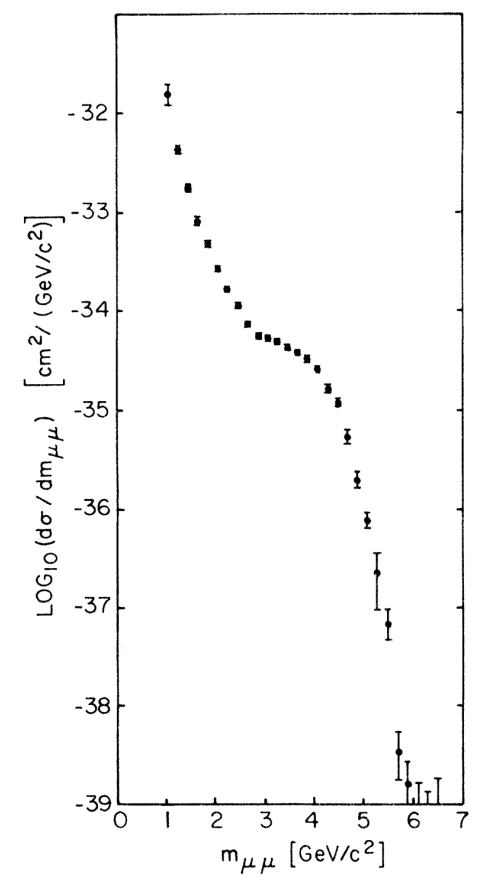

Figure 1.5: Cross section as a function of muon-pair mass $M_{\mu \mu}[58$. 


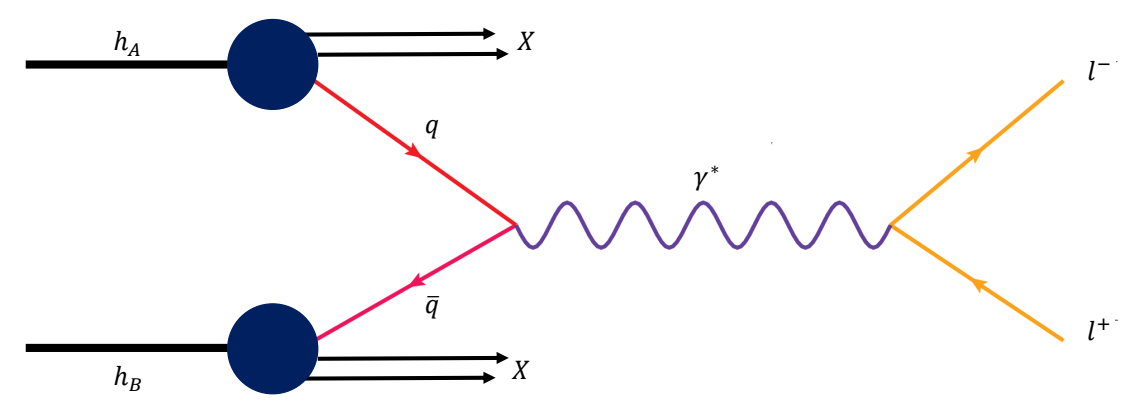

Figure 1.6: Illustration of the Drell-Yan process.

\subsubsection{Kinematics of the Drell-Yan Process}

Generally, the Drell-Yan process is the production of lepton pairs (dileptons) originating from the combination of partons from colliding hadrons, via an intermediate virtual boson state. The lepton pairs can be either $e^{-} e^{+}, \mu^{-} \mu^{+}$, or $\tau^{-} \tau^{+}$, and the virtual boson is dominantly a photon if the effective mass of the dilepton, $M_{l^{-} l^{+}}$, is much smaller than the $\mathrm{Z}$ boson mass $(\approx 91 \mathrm{GeV})$. At leading order, the essential steps for a Drell-Yan process is a quark $q$ from the hadron $h_{A}$ annihilates with an antiquark $\bar{q}$ from $h_{B}$, a virtual time-like photon $\gamma^{*}$ is subsequently produced and turns into a lepton pair $l^{-} l^{+}$. The process is illustrated as a Feynman diagram in Fig. 1.6.

Experimentally, as an inclusive measurement, only the dileptons in the final stage of the DrellYan process are detected. All the other hadrons produced, which are denoted as $X$ in Fig. 1.6. are simply neglected. The kinematics of the Drell-Yan process are therefore determined by six quantities from the dileptons that can be directly measured, namely the three-momenta of each lepton. Based on energy-momentum conservation, these six measurables can be translated into a different set of six independent kinematic variables. Knowing that the effective properties of the dilepton are identical to the virtual photon, four out of the six variables are in general chosen as the 


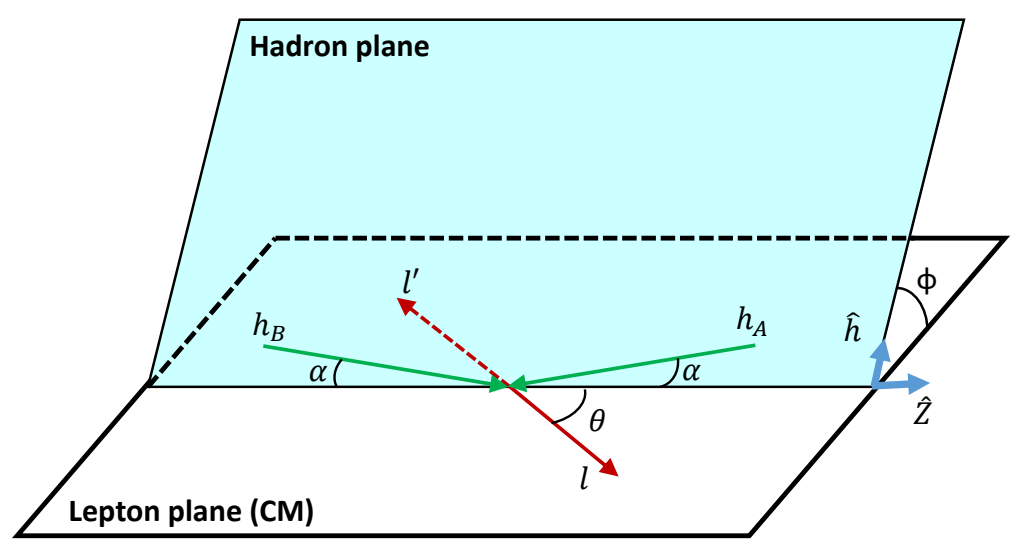

Figure 1.7: The definitions of $\theta$ and $\phi$ in the Collins-Soper (CS) frame. CS frame is a center of mass frame of the dilepton produced in a hadron-hadron collision, in which the Z-axis points along the bisector of one and opposite of the other of the directions of the incoming hadrons. The hadrons are denoted as $h_{A}$ and $h_{B}$, and the unit vector $\hat{h}$ lies on the hadron plane while being perpendicular to $\hat{Z}$. The leptons produced are labeled as $l$ and $l^{\prime}$.

ones describing the virtual-photon in the hadron-hadron center-of-mass frame with the Z-axis lying along the beam direction: the mass $M_{\gamma^{*}}=M_{l^{-} l^{+}}$, the Feynman-x $x_{F}$ which represents the fraction of the maximum possible longitudinal ( $\mathrm{Z}$ direction) momentum carried by the virtual photon, the transverse momentum $p_{T}$, and the azimuthal production angle $\phi_{\gamma^{*}}$. The two variables left are used to specify the angles of the dilepton produced in the virtual-photon rest frame. Conventionally, they are defined as the polar angle $\theta$ and the azimuthal angle $\phi$ in the Collins-Soper (CS) frame, as illustrated in Fig. 1.7 .

The virtual photon mass, $M_{\gamma^{*}}$, and the Feynman-x, $x_{F}$, can be converted to another two variables that are relevant to the annihilating quark and antiquark. These two variables are Bjorken-x, which is the momentum fraction of the nucleon carried by the annihilating quark or antiquark. Suppose the quark (antiquark) from hadron $h_{A}$ has a momentum fraction $x_{A}$ of $h_{A}$ and the antiquark (quark) from $h_{B}$ carries $x_{B}$. Then in the center-of-mass frame at the high longitudinal momentum limit, the masses of particles and their transverse momentum are neglected and the magnitude of the longitudinal momentum of the two hadrons are equally $\sqrt{s} / 2$, where $s$ is the center-of-mass 
energy squared of the hadrons. The annihilating pair thus have longitudinal momentum of $x_{A} \sqrt{s} / 2$ and $-x_{B} \sqrt{s} / 2$, respectively. The energy $E$ and longitudinal momentum $p_{\gamma^{*}}$ of the virtual photon are consequently

$$
E=\left(x_{A}+x_{B}\right) \sqrt{s} / 2
$$

and

$$
p_{\gamma^{*}}=\left(x_{A}-x_{B}\right) \sqrt{s} / 2 .
$$

$M_{\gamma^{*}}^{2}$ is then given as

$$
M_{\gamma^{*}}^{2}=E^{2}-p_{\gamma^{*}}^{2}=x_{A} x_{B} s .
$$

Since the maximum $p_{\gamma^{*}}$ corresponds to $x_{A}=1$ and $x_{B}=0$, by Eq. $1.9 x_{F}$ can be derived as

$$
x_{F}=2 p_{\gamma^{*}} / \sqrt{s}=x_{A}-x_{B} .
$$

By introducing another dimensionless variable $\tau=M_{\gamma^{*}}^{2} / s=x_{A} x_{B}$, the Bjorken-x's can be expressed as

$$
x_{A}=\frac{1}{2}\left(\sqrt{x^{2}+4 \tau}+x_{F}\right), x_{B}=\frac{1}{2}\left(\sqrt{x^{2}+4 \tau}-x_{F}\right) .
$$

Note that in E906, with $\sqrt{s} / 2 \sim 15 \mathrm{GeV}$, the assumption of $\sqrt{s} / 2$ being much greater than particle masses and $p_{T}$ in the previous derivation is not always valid. Nevertheless, the equations above hold approximately, with corrections less than $1 \%$ in general. The eight kinematic variables, $x_{A}, x_{B}, x_{F}, M_{\gamma^{*}}, p_{T}, \theta$, and $\phi$, that have been given in the discussion above are going to be frequently used in the rest of the thesis. Being the only relevant mass term, $M_{\gamma^{*}}$ will be abbreviated as $M$ hereafter. The Bjorken-x's, rather than $x_{A}$ and $x_{B}$, will be written in another commonly used form as $x_{1}$ and $x_{2}$ simply to follow the convention adopted in the SeaQuest experiment; in fixed-target experiments, $x_{1}$ is generally used for beam parton and $x_{2}$ for target parton.

\subsubsection{Cross Section of the Drell-Yan Process}

The Drell-Yan cross section, at leading order (LO), comes in a factorized form that is fairly straightforward to understand. The hard-interaction portion of the Drell-Yan cross section is de- 
termined by the electromagnetic quark-antiquark annihilation

$$
q+\bar{q} \rightarrow \gamma^{*} \rightarrow l^{-}+l^{+}
$$

and the relevant hard scale $Q$ is the mass of the dilepton $M$. Similar to electron-positron annihilation, the cross section of this subprocess can be calculated via QED as

$$
\hat{\sigma}=4 \pi \alpha_{e}^{2} e_{q}^{2} / 3 M^{2}
$$

where the $\alpha_{e}$ is the electromagnetic fine structure constant, $e_{q}$ the quark charge, and $M$ the dilepton mass. On the other hand, the long-distance part related to the initial-state hadrons is given via distribution functions. By the Heisenberg uncertainty principle, the time scale of the annihilation process is inversely proportional to the hard scale $Q$. Therefore, with high $Q$ and short interaction period, the partons behave as if they were "frozen" during the interaction. In general, the minimum $Q$ is considered to be on the order of a few $\mathrm{GeV}$, which corresponds to a time scale of $\sim 10^{-25} \mathrm{~s}$, or equivalently a distance scale of $\sim 10^{-2} \mathrm{fm}$ (the nucleon size is $\sim 1 \mathrm{fm}$ ). The incoming hadrons can therefore be regarded as a collection of free, non-interacting partons, with the probability of being in a particular state described by their PDFs. Therefore, the Drell-Yan cross section is the product of the quark PDF, antiquark PDF, and the cross section $\hat{\sigma}$.

Let the quark distribution function of flavor $q$ from hadron $h_{A}$ denoted as $q_{A}\left(x_{1}\right)$, which gives the probability of this quark carrying momentum fraction $x_{1}$. Likewise, denote the antiquark of the same flavor in hadron $h_{B}$ as $\bar{q}_{B}\left(x_{2}\right)$. By flavor conservation, the flavor of the annihilating pair must match to produce a flavor-less virtual photon. Also, conservation of color introduces a factor of $1 / 3$ to the cross section. The Drell-Yan cross section for a quark of flavor $q_{A}$ at $x_{1}$ annihilating with the antiquark $\bar{q}_{B}$ with $x_{2}$ is

$$
d^{2} \sigma=\frac{4 \pi \alpha_{e}^{2}}{9 s x_{1} x_{2}} e_{q}^{2}\left[q_{A}\left(x_{1}\right) \bar{q}_{B}\left(x_{2}\right)\right] d x_{1} d x_{2}
$$

Since the lepton pair with identical kinematics can also be produced by a antiquark from $h_{A}$ and quark from $h_{B}$, an additional term with $q$ replaced as $\bar{q}$ and $\bar{q}$ as $q$ in Eq. 1.15 needs to be put in. 
Finally, by summing all possible quark flavors, the differential cross section is

$$
\frac{d^{2} \sigma}{d x_{1} d x_{2}}=\frac{4 \pi \alpha_{e}^{2}}{9 s x_{1} x_{2}} \sum_{q \in\{u, d, s, \ldots\}} e_{q}^{2}\left[q_{A}\left(x_{1}\right) \bar{q}_{B}\left(x_{2}\right)+\bar{q}_{A}\left(x_{1}\right) q_{B}\left(x_{2}\right)\right],
$$

By the relation given in the previous subsection, this equation can also be re-expressed as the differential cross section of $M^{2}$ and $x_{F}$ :

$$
\frac{d^{2} \sigma}{d M^{2} d x_{F}}=\frac{4 \pi \alpha_{e}^{2}}{9 M^{4}} \frac{x_{1} x_{2}}{x_{1}+x_{2}} \sum_{q \in\{u, d, s, \ldots\}} e_{q}^{2}\left[q_{A}\left(x_{1}\right) \bar{q}_{B}\left(x_{2}\right)+\bar{q}_{A}\left(x_{1}\right) q_{B}\left(x_{2}\right)\right],
$$

where

$$
x_{1}=\frac{1}{2}\left[\left(x_{F}^{2}+4 \tau\right)^{1 / 2}+x_{F}\right], x_{2}=\frac{1}{2}\left[\left(x_{F}^{2}+4 \tau\right)^{1 / 2}-x_{F}\right],
$$

with $\tau=M^{2} / s$, identical to the definitions given in Eq. 1.12 ,

\subsubsection{Higher Order QCD Correction}

With higher order in $\alpha_{s}$ being considered, the complexity of Feynman diagrams and the calculation involved grows quickly. However, some important features of the higher-order corrections brought by QCD can be observed by just studying the case in next-to-leading-order (NLO), order $\mathcal{O}\left(\alpha_{s}\right)$

The Drell-Yan diagrams at order $\mathcal{O}\left(\alpha_{s}\right)$ are shown in Fig. 1.8, The diagrams in (a) can be regarded as the vertex correction and the contribution to the quark self-energy. The diagrams of (b) are sometimes called as the "annihilation diagrams" and the ones in (c) as the "Compton diagrams". Due to the recoiling quarks or gluons in diagrams of (b) and (c), large transverse momentum of the virtual photon can arise. As shown in [59 61], the cross section calculation including all these diagrams would have terms that are logarithmic in the square of the hard scale $Q$. Together with other pieces, the $\ln Q^{2}$ terms can be combined with the distribution functions $q(x)$ or $\bar{q}(x)$ to give $Q^{2}$-dependent distribution functions that have the same form as the ones appearing in DIS, with $Q^{2}$ replaced by $\left|Q^{2}\right|$ since the virtual photon in DIS is space-like. With these scale-dependent

distribution functions $q\left(x, Q^{2}\right)$ and $\bar{q}\left(x, Q^{2}\right)$, the cross section can be put in an expression that is somewhat similar to the leading-order one with additional corrections. These corrections are often 


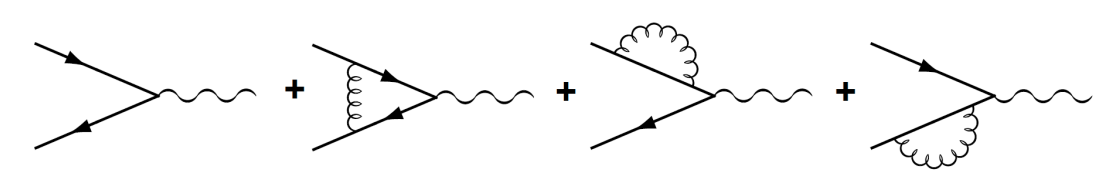

( a )

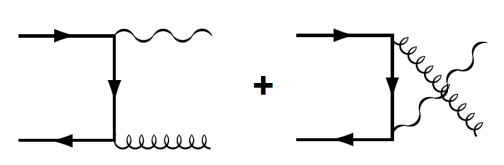

(b)

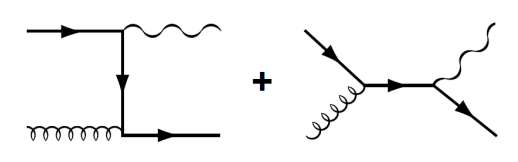

(c)

Figure 1.8: Feynman diagrams of Drell-Yan to the order $\mathcal{O}\left(\alpha_{s}\right)[62]$.

approximated as a single overall correction factor as

$$
1+\frac{\alpha_{s}}{2 \pi} \cdot\left(\frac{4}{3} \pi\right)^{2}+\ldots
$$

which is called the $K$-factor. Notice that the $K$-factor is rather large due to the $\pi^{2}$ correction term; for an $\alpha_{s}$ of about 0.3 , the $K$ factor is approximately 2 . While the complete non-leading corrections cannot be expressed exactly as an overall factor, the $K$-factor is commonly adopted as an approximation to represent the relative significance of higher-order corrections to the leading-order cross section.

Beyond NLO, factorizability for the Drell-Yan process has be proven to all orders of $\alpha_{s}$. The QCD corrections up to $\mathcal{O}\left(\alpha_{s}^{2}\right)$ have been explicitly calculated [63 65] and factorization has been confirmed. The relevant theoretical achievements, combined with measurements, have made the Drell-Yan process a powerful probe for exploring the structure of nucleons, nuclei, and even unstable particles. Through the so-called "global PDF fits" that incorporates pQCD calculations of corresponding partonic cross sections to the same order of $\alpha_{s}$ as desired, there have been analysis groups trying to extract PDFs by utilizing experimental results from various processes at different energy scales, such as NNPDF [66, 67, MMHT 68, 69], CTEQ-TEA [70, 71], HERAPDF [72, 73], 

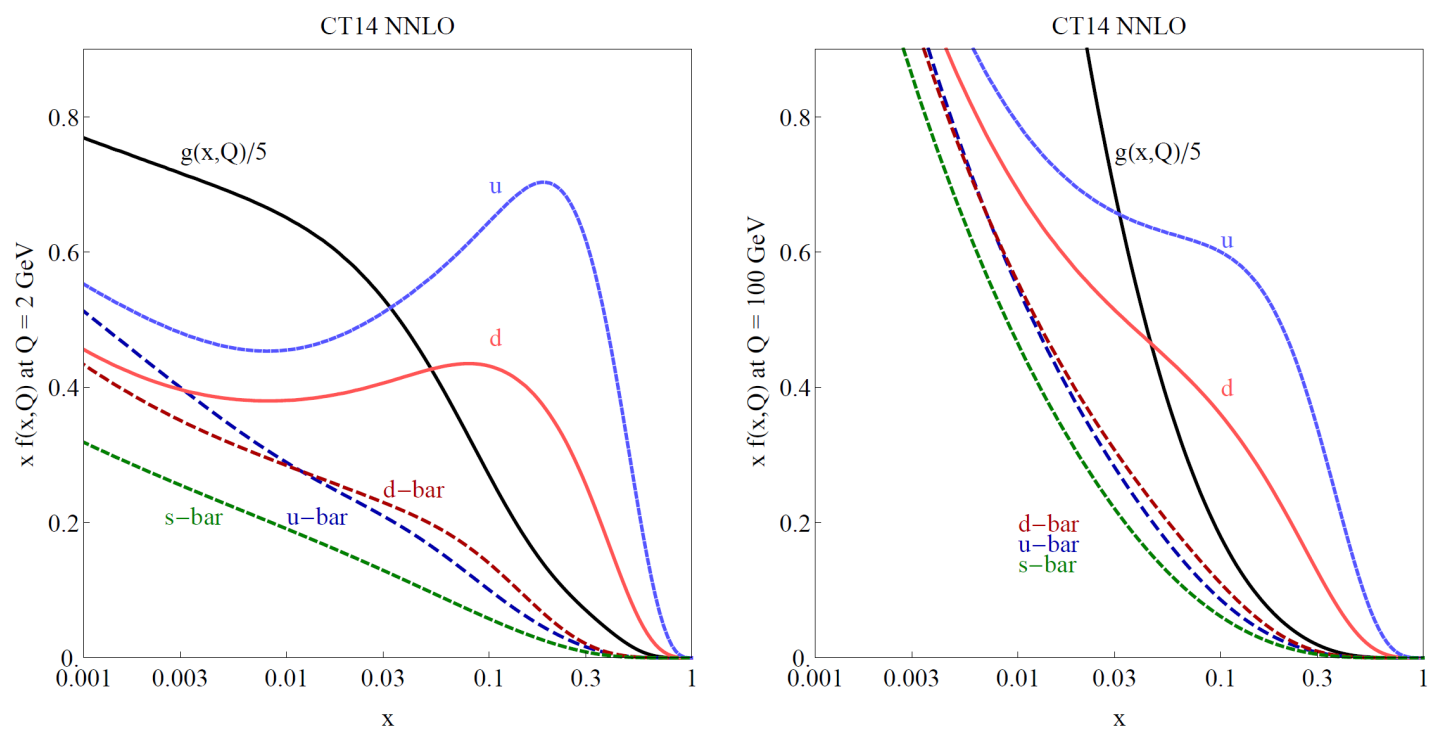

Figure 1.9: NNLO parton distribution functions at $Q=2 \mathrm{GeV}$ (left) and $Q=10 \mathrm{GeV}$ (right) from the CTEQ-TEA global analysis (CT14). On the Y-axis, the PDF is denoted as $f(x, Q)$.

CTEQ-JLab [74, 75], and ABMP16 [76]. As an example, the PDFs at next-to-next-to-leading order (NNLO) from the CTEQ-TEA global analysis are shown in Fig. 1.9. Other than HERAPDF, all the groups above included data from Drell-Yan process, and SeaQuest is expected to provide crucial input to further constraint the sea-quark PDF at high Bjorken-x.

\subsection{Energy Loss Measurements with Drell-Yan}

Parton energy loss in the Drell-Yan interaction is an initial-state process, which can be intuitively seen by the illustration in Fig. 1.10. In this figure, as the fast parton from the projectile hadron propagates through the cold nuclear matter, which can be a heavy nucleus, it can suffer energy loss before the annihilation takes place. Due to the fact that the dimuons produced do not interact strongly with the nuclear medium, final-state interactions can be ignored. This makes the Drell-Yan process an ideal probe for the energy loss since its effect can be cleanly observed through the dimuons.

Because of energy loss, the energy of the incoming parton right before annihilation would 


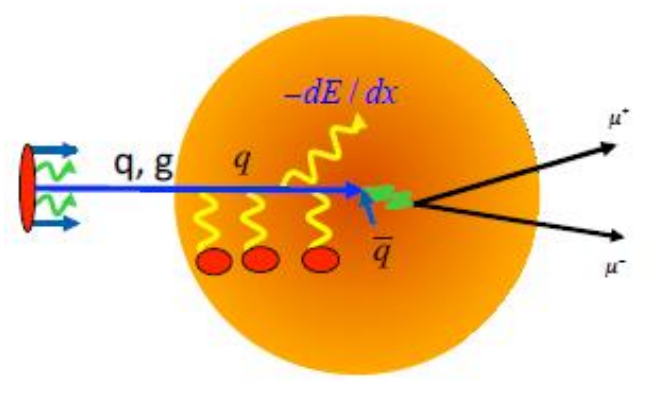

Figure 1.10: Energy loss process in Drell-Yan interaction. The incoming parton loses energy in the heavy nucleus before the annihilation with another parton in the nucleus.

be different from its initial value. This shift in energy can be equivalently expressed as a change in the apparent hadron momentum fraction carried by the parton participating the annihilation process. Therefore, similar to what has been observed in heavy-ion collisions, one would expect that a signature of initial-state energy loss is the modification in the dimuon-production spectrum with respect to Bjorken-x or $x_{F}$. And since the magnitude of energy loss is presumably larger in heavier nuclei, by measuring the nuclear dependence of the proton-nucleus $(p-A)$ Drell-Yan differential cross sections, the energy losses can be extracted.

Here a heuristic phenomenological illustration as discussed in 53] is given to demonstrate how the energy loss effect can be accessed by the ratio of proton induced Drell-Yan cross section from nuclear target $A$ to that from deuterium, in a fixed-target experiment employing detectors of very forward acceptance like E906/SeaQuest. With this kind of detector geometry, only dilepton pairs with high $x_{F}$, originating from beam parton of high $x_{1}$ and target parton of low $x_{2}$, are accepted. As indicated by the Bjorken-x dependence of the PDFs shown in Fig. 1.9, the measured Drell-Yan cross section can be approximated as arising only from a beam quark annihilating with a target anti-quark, which corresponds to the first term in the square bracket of Eq. 1.16. Denote the energy of the incoming proton as $E_{p}$, and consider the case in which the small value of $x_{2}$ falls in a limited range that makes $\bar{q}^{A}\left(x_{2}\right) / \bar{q}^{D}\left(x_{2}\right) \simeq 1$ (such as $0.1<x_{2}<0.3$, as suggested in [77]), where 
the superscripts specify the anti-quark PDF of either nucleus $A$ or deuterium. Then by Eq. 1.16, the $u\left(x_{1}\right) \bar{u}\left(x_{2}\right)$ term dominates due to the 4 to 1 charge-square weighting and the $\sim 2$ to $1 u$ to $d$ ratio in the incident proton at high $x_{1}$. The ratio of the per-nucleon differential cross section with nucleus $A$ to the one of deuterium can thus be approximated as

$$
\frac{1}{A_{A}}\left(\frac{d^{2} \sigma_{p A}}{d x_{1} d x_{2}}\right) / \frac{1}{A_{D}}\left(\frac{d^{2} \sigma_{p D}}{d x_{1} d x_{2}}\right) \simeq \frac{q_{u}^{p}\left(x_{1}^{A}\right)}{q_{u}^{p}\left(x_{1}^{D}\right)} \simeq \frac{\left(1-x_{1}^{A}\right)^{3}}{\left(1-x_{1}^{D}\right)^{3}}
$$

where the $A$ 's are the mass numbers, and the approximate $(1-x)^{3}$ behavior of $q_{u}^{p}\left(x_{1}\right)$ is in the $x_{1} \rightarrow 1$ limit $\left(x_{1} \gtrsim 0.5\right)$, as given by quark counting rules. Assume the quark energy loss $\Delta E$ in nuclear matter is linearly dependent on the average path length $\langle L\rangle_{A}$ traveled by the quark, and can be written as $\Delta E=\kappa\langle L\rangle_{A}$. The apparent $x_{1}^{A}$ for the quark-antiquark annihilation in the nuclear target would consequently be shifted by $\Delta x_{1}^{A}=\Delta E / E_{p}=\kappa\langle L\rangle_{A} / E_{p}$, compared to the case of deuterium where presumably there is little energy loss. In Eq. 1.19 the $x_{1}^{A}$ dependence of the PDF part can thus be written as

$$
x_{1}^{A}=x_{1}^{D}+\frac{\kappa\langle L\rangle_{A}}{E_{p}},
$$

since the apparent $x_{1}^{A}$ must originate from a value larger than $x_{1}^{D}$. To the leading order in $\kappa\langle L\rangle_{A} / E_{p}$, Eq. 1.19 can then be expressed as

$$
\left(\frac{d^{2} \sigma_{p A}}{d x_{1} d x_{2}}\right) /\left(\frac{d^{2} \sigma_{p D}}{d x_{1} d x_{2}}\right) \simeq \frac{\left(1-x_{1}^{D}+\frac{\kappa\langle L\rangle_{A}}{E_{p}}\right)^{3}}{\left(1-x_{1}^{D}\right)^{3}} \simeq 1-\frac{3 \kappa\langle L\rangle_{A}}{E_{p}\left(1-x_{1}^{D}\right)} .
$$

As can be seen in Eq. 1.21, a key feature in the cross-section ratio brought by energy loss is the suppression at large $x_{1}$. With greater energy-loss or lower beam proton energy $E_{p}$, the suppression would be more significant. Although the derivation above is based on the leading-order Drell-Yan cross section, the behavior of the cross-section ratio observed still holds with high-order corrections since the overall K-factors are expected to simply cancel [53, 54]. Indeed, a fair amount of approximation was employed in the previous discussion and at least a more-careful treatment on the PDFs are required for a quantitative analysis. Particularly, one needs to be cautious with the assumption of $\bar{q}^{A}\left(x_{2}\right) / \bar{q}^{D}\left(x_{2}\right) \simeq 1$, since the PDF of a bounded nucleon can be noticeably modified due to nuclear effects. 


\subsubsection{Nuclear Dependence of Parton Distributions}

The fact that the distribution of partons of a bounded nucleon in a heavy nucleus is different from that of a free nucleon was demonstrated for the first time by the European Muon Collaboration (EMC) in 1983 [78]. They measured the per-nucleon muon DIS cross-section ratio between iron and deuterium, which is approximately the ratio of structure functions $F_{2}^{F e} / F_{2}^{D}$, where $F_{2}$ is defined as the sum of the distribution functions weighted by the charge and Bjorken-x (abbreviated as $x$ in this subsection)

$$
F_{2}\left(x, Q^{2}\right)=\sum_{q \in\{u, d, s, \ldots\}} x e_{q}^{2}\left[q\left(x, Q^{2}\right)+\bar{q}\left(x, Q^{2}\right)\right]
$$

in which the definition of variables is similar to the one used in Eq. 1.16. Rather than the rapid rise with $x$ as theoretically predicted based on the nucleon Fermi motion, a monotonic drop was observed. This discovery by EMC showed a surprising nuclear dependence of the parton distribution and triggered many studies pursuing a full characterization of this phenomenon.

To date, a large amount of data has been accumulated by charged-lepton DIS experiments, and a universal $x$ dependence of $\sigma^{A} / \sigma^{D}$ among various nuclear targets $A$ has been identified. As illustrated in Fig. 1.11 for example, this universal $x$ dependence can be subdivided into four separate regions as:

- $0<x<x_{1} \bumpeq 0.06$ : The cross-section ratio is less than one and gets smaller with decreasing $x$ in this so-called "shadowing region". The term shadowing was introduced to explain the reduction of the cross-section ratio: Naïvely, in a heavy nucleus, some nucleons are "shadowed" by the nucleons in the front and are less likely to participate the interaction.

- $x_{1}<x<x_{2} \simeq 0.3$ : In the "anti-shadowing region", which is generally regarded as a transition region, the ratio is a few-percent greater than one.

- $x_{2}<x<x_{3} \simeq 0.8$ : This is the region exhibiting the "EMC effect", named after the EMC collaboration. The ratio decreases as $x$ increases and reaches its minimum around $x \approx 0.7$. This declining trend should be attributed to the valence-quark distributions since 


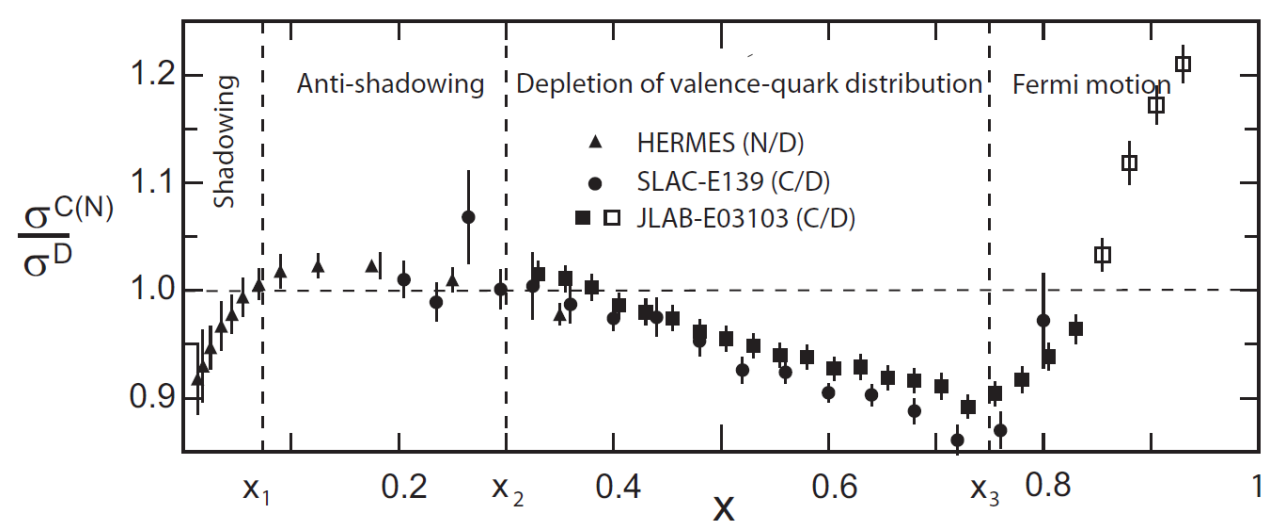

Figure 1.11: Measured per-nucleon cross section ratio $\sigma^{C(N)} / \sigma^{D}$ from HERMES [79], SLACE139 [80], and JLAB-E03103 [81]. This plot is from [82].

the sea-quark distribution is small (see Fig. 1.9).

- $x_{3}<x<1$ : With increasing $x$, the ratio shows a rapid rise. This behavior has been interpreted as a convolution of effects from the Fermi motion of a bounded nucleon and the vanishing free-nucleon cross section as $x$ approaches 1 .

Although the nuclear dependence of parton distributions has been measured over a wide range of different kinematic variables, the origin is still not fully understood. Phenomenologically, more insight into this puzzle can be provided by measuring the effective nuclear parton distribution functions (nPDFs) via procedures assuming factorization and universality. Traditionally, the $\mathrm{nPDF}$ are quantified by the nuclear modification factor $R_{i}^{A}\left(x, Q^{2}\right)$, a parameterized multiplicative correction factor, which is conceptually similar to the one mentioned in Eq. 1.6 and is defined as

$$
R_{i}^{A}\left(x, Q^{2}\right)=f_{i}^{A}\left(x, Q^{2}, A\right) / f_{i}^{p}\left(x, Q^{2}\right),
$$

where $f_{i}^{A}\left(x, Q^{2}\right)$ is the nPDF in a nucleus of atomic number $A$ for a parton of flavor $i$, and $f_{i}^{p}\left(x, Q^{2}\right)$ is the corresponding proton PDF. Similar to global PDF fit, there exists several collaborations analyzing nPDFs by incorporating different data with specific parameterization. Fig. 1.12 shows a number of extracted nuclear modification factors by different collaborations and it can be observed that all the factors for the sea quarks in the small- $x$ shadowing region are noticeably smaller than 


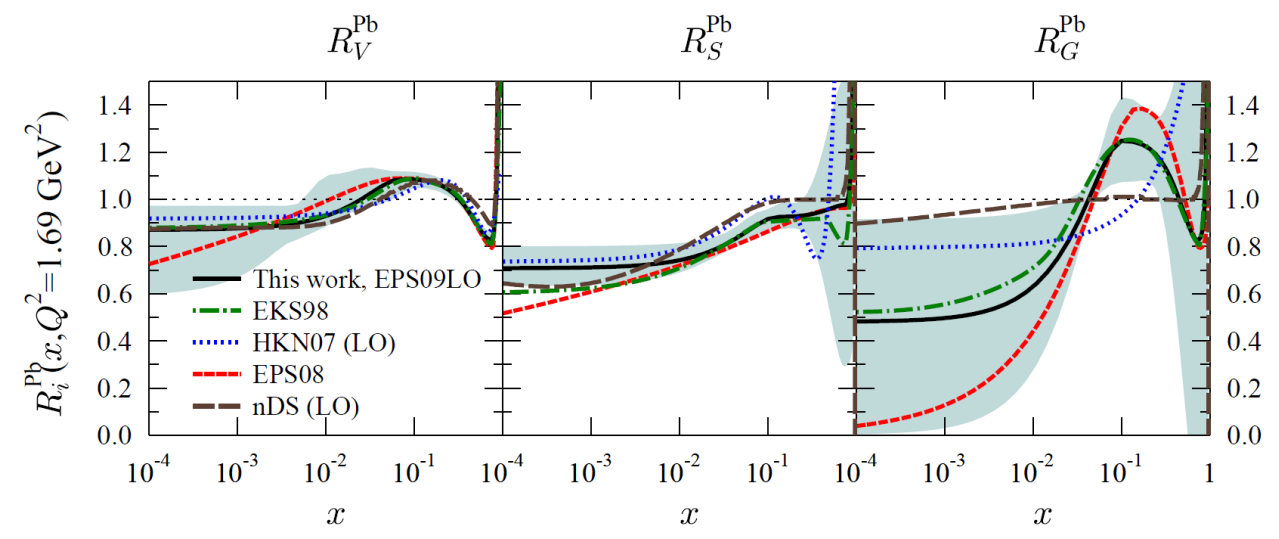

Figure 1.12: Nuclear modification factors of the average valence and sea quarks, and the gluons in $\mathrm{Pb}$ nucleus at $Q^{2}=1.69 \mathrm{GeV}^{2}$ from EKS98 [83, 84], EKPS [85], nDS [86], HKN07 [87], and EPS09LO [88. The green bands represent the estimated uncertainty band from the EPS09LO results.

unity. As mentioned above, since fixed-target Drell-Yan experiments would favor antiquarks of relative low $x_{2}$ from targets, the suppression of cross-section ratio at high $x_{1}$ could also arise from nuclear shadowing. To precisely determine the energy loss, special attention to the shadowing effect is required.

\subsubsection{Previous Measurements}

The Fermilab experiments, E772 and E866, are the two predecessors of E906 and both investigated the nuclear dependence of proton-induced Drell-Yan production of muon pairs by using the $800 \mathrm{GeV}$ proton beam at Fermilab. E866 made their energy loss measurement by analyzing the cross-section ratio of iron and tungsten over beryllium versus $x_{1}$ [89]. Since a large portion of the E866 data comes with $x_{2}<0.05$, which falls in the region of significant nuclear shadowing, the EKS98 [83, 84] nPDF parameterization was adopted to correct the shadowing effect. After compensating for the suppression from shadowing, three theoretical parameterizations [90 93] of different kinematic dependency were then used for energy loss extraction, as shown in Fig 1.13 . What E866 found was a very small energy-loss rate $(<0.44 \mathrm{GeV} / \mathrm{fm})$ that is consistent with no 


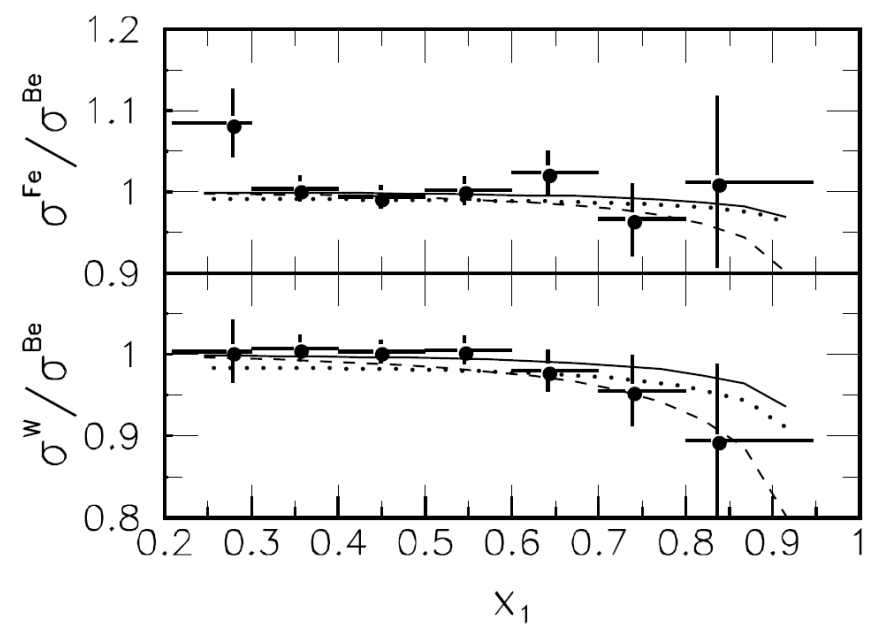

Figure 1.13: Per-nucleon cross-section ratio of $\mathrm{Fe} / \mathrm{Be}$ and $\mathrm{W} / \mathrm{Be}$ from E866 with shadowing corrected [89]. With form indicated in [90], the best fits are represented by the solid curves and the 1- $\sigma$ upper limits are shown in dashed curves. The dotted curves gives the 1- $\sigma$ upper limits with formulation give in [92, 93].

energy loss observed. This result, however, is inconclusive [53, 94, 95] owing to the fact that the EKS98 analysis included E772 data [77] for determining the sea-quark shadowing without taking parton energy loss into account. Utilizing EKS98 for the shadowing correction could naturally lead to a smaller energy loss, compared to that if a different nPDF set were employed, as suggested in [54].

The data from E772 and E866 were later analyzed by Johnson et al. [94, 96] and a restframe based calculation of the process was used. In their formulation of the Drell-Yan process, the lepton pair originates from the decay of a heavy photon bremsstrahlunged by an incident quark. The shadowing is related to the lifetime of the virtual fluctuation of the incoming quark into the massive photon and quark, $q \leftrightarrow q \gamma^{*}$, and was claimed to produce considerably less suppression. A greater energy loss was consequently obtained, as shown in Fig. 1.14, and the measured energy loss rate is more than $2 \mathrm{GeV} / \mathrm{fm}(2.73 \pm 0.37 \pm 0.5 \mathrm{GeV} / \mathrm{fm}$ from [96]). As pointed out by [53], this rather large value is partly due to the short parton path length used in the analysis, since the incident proton needs to travel a certain distance (mean-free-path $\sim 2.5 \mathrm{fm}$ ) in the nuclear matter before a energetic 

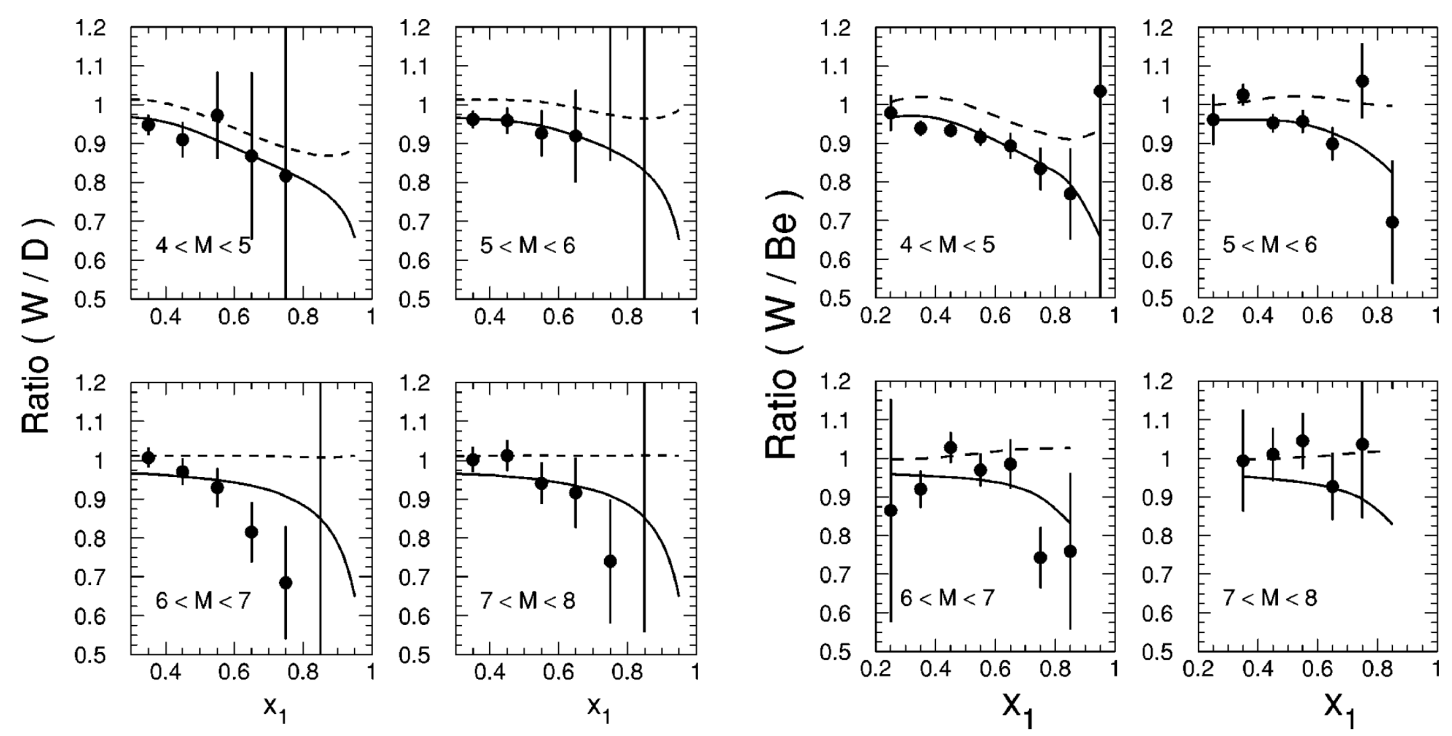

Figure 1.14: Per-nucleon cross-section ratio of W/D from E772 (left) and W/Be from E866 (right) in different mass bin, with shadowing estimation from [96]. The shadowing contribution are given as dashed curves and the full effect including energy loss and shadowing are shown in solid curves.

parton can be liberated. But even when using the conventional path length of $3 / 4\left(1.2 A^{1 / 3}\right) \mathrm{fm}$, one would still obtain a energy loss rate of about $1 \mathrm{GeV} / \mathrm{fm}$. This observation was later questioned for the reason that although the approach employed the shadowing correction describing the DIS data fairly well at small $x_{2}$, it failed to reproduce the observed nuclear dependence for $x_{2}>0.04$, where most of the E772 data and nearly $50 \%$ of E866 data reside 97 .

Other than proton induced Drell-Yan, Arelo [95] studied energy loss from the NA3 data of $\pi^{-}$-A Drell-Yan production [98] utilizing $150 \mathrm{GeV}$ pion beam. The mean $x_{2}$ of data collected by NA3 falls between 0.06 and 0.3 , and only tiny (anti-)shadowing effect was expected. The energy loss rate was found to be $0.20 \pm 0.15 \mathrm{GeV} / \mathrm{fm}$, which shows a large disagreement with the one from [96]. Nevertheless, it has been argued that this large discrepancy might be attributed to the low statistics of NA3 data and the not-well-understood parton distributions of pion [53].

From the discussion above, one can see that the problem of reliably determining the parton energy loss in cold nuclear matter largely comes from the obscurity brought by nuclear effects, and 
the limited accessible experimental data. To resolve this, a new experiment, which addresses the energy loss measurement and has a kinematic coverage in which other nuclear modifications are not expected to have strong contributions, is desired.

\subsubsection{E906/SeaQuest Experiment}

With the $120 \mathrm{GeV}$ proton beam from the Fermilab main injector, E906 measures the production of Drell-Yan and $J / \psi$ production in the dimuon channel in p-p and p-A collisions, utilizing targets of H, D, C, Fe, and W. E906 has been collecting high statistics data since 2014 and the timeline of E906 is given in Tab. 1.1. With the substantially lower beam energy compared to E772 and E886, the expected energy-loss effect via the cross-section ratio should be amplified significantly. Furthermore, the experiment measures events with $0.1<x_{2}<0.45$, which is comfortably above the shadowing domain. However in this $x_{2}$ range, other nuclear modifications, such as EMC effect and anti-shadowing, of anti-quarks has not been well established yet. The previous measurements of E772 showed little or even no nuclear dependence except in the shadowing region, and this will be further verified by the high-statistics data from E906.

There have been theoretical works that made specific prediction for SeaQuest. Xin et al. calculated the differential cross section within the HT formalism [99]. The DGLV approach was adopted by Neufeld et al. [100] and the predicted cross-section ratio is shown in Fig. 1.15. Both of the studies showed negligible shadowing effect and a significant attenuation of the cross-section ratio, thus demonstrating that E906 has a good opportunity to precisely measure the energy loss in cold nuclear matter.

The rest of this thesis is organized as follows. In Chapter 2, the E906/SeaQuest experiment is introduced. The details of data analysis for the energy loss measurement is presented in Chapter

3 , followed by the results and discussions shown in Chapter 4. Finally, the summary is given in Chapter 5 . 


\begin{tabular}{ll}
\hline Dates & Status \\
\hline 2009 Mar. - 2012 Mar. & Construction \\
2012 Mar. - 2012 Apr. & Run I - detector commissioning \\
2012 Apr. - 2013 Nov. & Detector upgrade \\
2013 Nov. - 2014 Aug. & Run II - physics run \\
2014 Aug. - 2014 Nov. & Accelerator shutdown \\
2014 Nov. - 2015 Jul. & Run III - physics run \\
2015 Jul. - 2015 Nov. & Accelerator shutdown \\
2015 Nov. - 2016 Jul. & Run IV - physics run \\
2016 Jul. - 2016 Nov. & Accelerator shutdown \\
2016 Nov. - 2017 Jul. & Run V - physics run \\
\hline
\end{tabular}

Table 1.1: Timeline of SeaQuest.

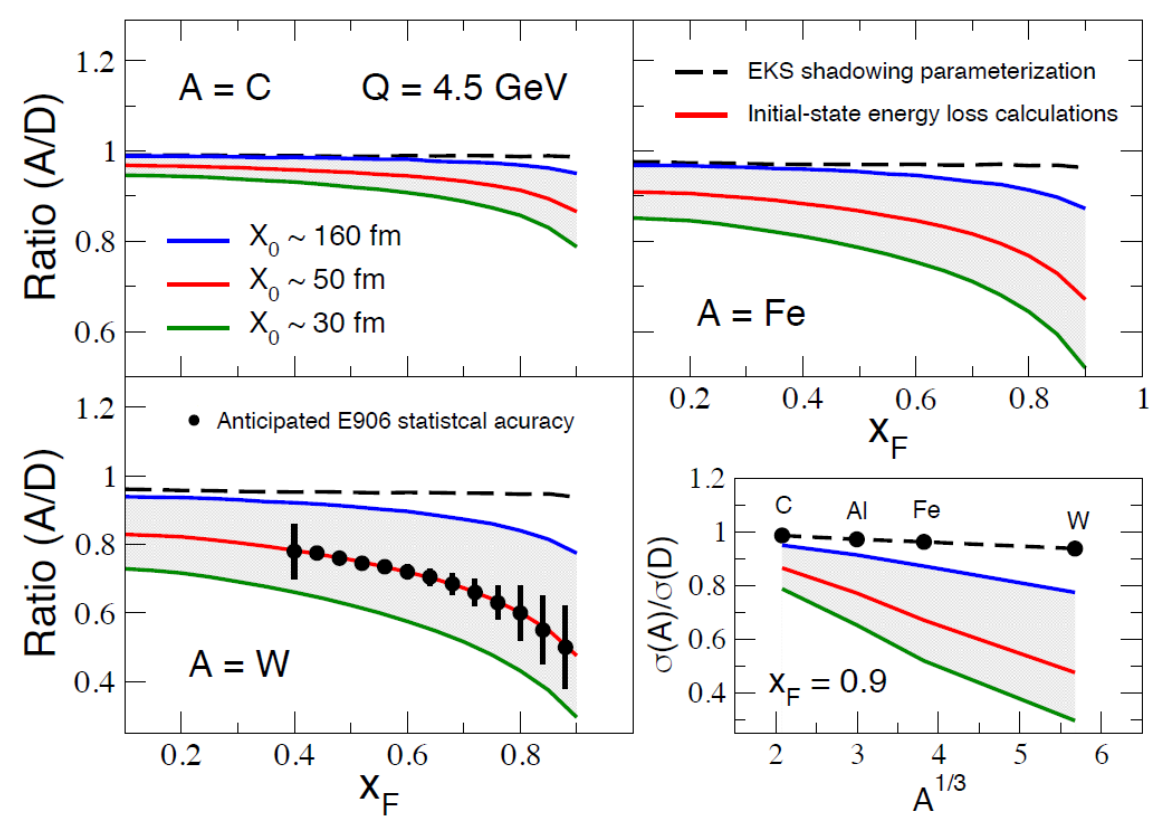

Figure 1.15: Theoretical prediction of per-nucleon cross section ratio for E906 by [100]. The solid curves are the numerical estimations with different parameterization of the radiation length $X_{0}$, with shadowing effects included. The lower-right panel shows the predicted $A^{1 / 3}$ dependence of the ratio at high $x_{F}$. 


\section{Chapter 2}

\section{Experimental Apparatus}

The E906/SeaQuest experiment is designed to focus on the measurement of oppositely charged high energy muon pairs emerging from the Drell-Yan process. Being conducted at the NM4 experiment hall at Fermi National Accelerator Laboratory (Fermilab), the E906 experiment uses the proton beam from the Fermilab Main Injector, and employs the spectrometer setup similar to previous Fermilab Drell-Yan experiment predecessors, E772, E789, and E866/NuSea.

Fig. 2.1 shows a schematic of the E906 spectrometer. As the beam comes, it would first meet the upstream instrumentation package and then interact with the targets. The muons generated

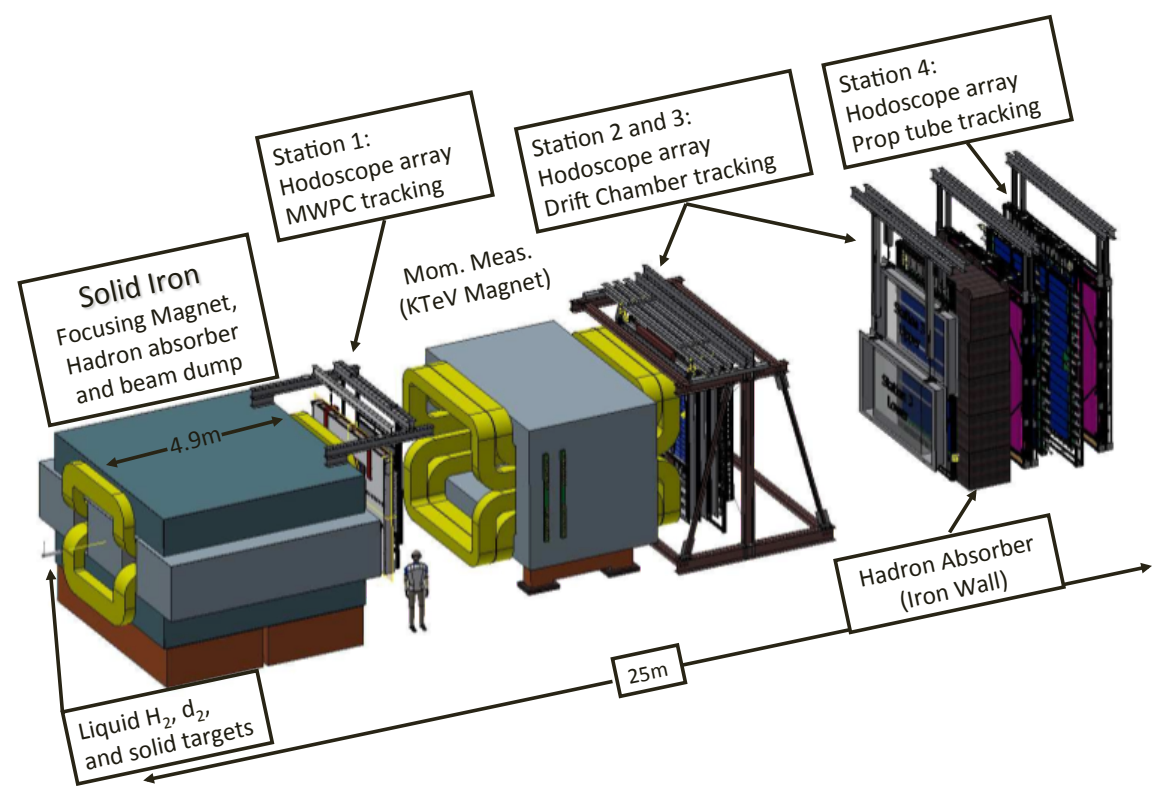

Figure 2.1: The E906/SeaQuest spectrometer. 
then go through the E906 spectrometer, which consists of two dipole magnets and four different tracking/triggering stations. Each station includes a set of drift chambers or proportional tube detectors for tracking, and hodoscope arrays providing fast information for trigger determination. Between station 3 and station 4 there is an iron wall that keeps background hadrons from reaching station 4 , and therefore station 4 is also used for muon identification.

The details of the experimental setup of E906 are described in this chapter. Throughout this chapter and the rest of the thesis, we will refer to the coordinate system that E906 has adopted. In this convention, the positive Z-axis points in the direction of the incoming beam and the positive Yaxis points vertically upward. The positive $\mathrm{X}$-axis is thus defined to form a right-handed Cartesian system with the origin set at the center of the front surface of the upstream magnet.

\subsection{Beam and Beam Monitors}

The accelerator complex of Fermilab is shown in Fig. 2.2. The proton beam used by SeaQuest is the slow extracted $120 \mathrm{GeV}$ beam from the Fermilab Main Injector. The Main Injector is a twomile-circumference ring which receives the $8 \mathrm{GeV}$ beam from the Booster and accelerates the beam protons to the energy of $120 \mathrm{GeV}$. The extraction of the beam from the Main Injector is performed using a resonant process, which brings instability to the circulating protons. An electro-magnetic septum can thus gradually scrape off the beam and have it directed out of the ring. This slow extraction lasts for about four seconds. Each extraction is referred to as a spill and the time between spills is typically just over 60 seconds.

The extracted beam is delivered to different beam lines dedicated for fixed-target experiments and the Fermilab Test Beam Facility. The Neutrino Muon beam line is one of the beam lines and the last two sectors of the beam line, NM3 and NM4, are used by E906. As the beam goes along the beam line, it passes through different detectors for different monitoring purposes. The position and size of the beam is measured by segmented wire ionization chambers (SWICs). Most of the SWICs are movable and are placed into the beam line only when beam tuning is carried out. There is one SWIC in NM3 that stays in the beam line which allows the status of the beam to be monitored on 


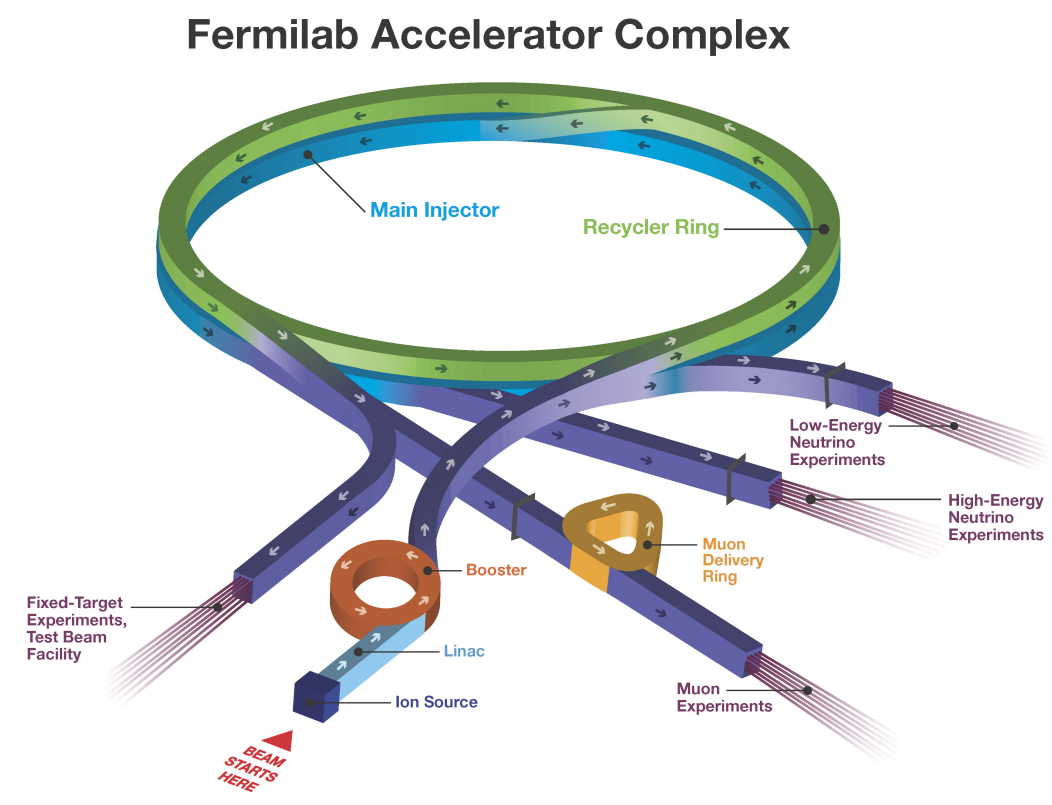

Figure 2.2: The accelerator complex of Fermilab [101].

a spill by spill basis.

The intensity of the beam is measured several times by ion chambers (ICs) and secondary emission monitors (SEMs). The SEM is preferred since it has a linear response over the proton intensity range that E906 is taking and doesn't saturate at high intensity as the ion chamber does. The SEMs are normalized by an activation measurement of a copper foil with a known cross section. For E906 the primary detector is the secondary emission monitor at the G2 sector of the beam line (G2SEM), and its readout has been used to evaluate the number of protons E906 received.

Before entering the target cave in NM3, the protons first pass through a SEM (NM3SEM), the SWIC mentioned above, and the Beam Intensity Monitor that will be discussed later. These together are referred to as the upstream instrumentation package and are viewed by a beam telescope at a roughly 90-degree angle relative to the beam direction. The beam telescope is a four-element scintillator detector which serves as an independent scattered-beam monitor, and it also triggers 
the sound alarm in the SeaQuest control room indicating an incoming spill.

The proton beam is bunched into small packets called buckets and retains the $53.1 \mathrm{MHz}$ RF structure of the accelerator. Typically, each bucket can be regarded as a cloud of protons, which is about $1 \mathrm{~cm}$ in diameter, $20 \mathrm{~cm}$ long, and separated from each other by about $18.8 \mathrm{~ns}$. On

average, there are about $10^{4}$ protons in each bucket and SeaQuest received a total of $10^{12}$ to $10^{13}$ protons per spill. The intensity of different buckets, however, can change significantly over a spill. For those very-high-intensity buckets, a large number of background tracks can be produced and therefore overwhelm the spectrometer, generating events that are practically unanalyzable, and also swamp the data acquisition system. To deal with this issue, a specific Beam Intensity Monitor was designed.

\subsubsection{Beam Intensity Monitor}

The Beam Intensity Monitor (BIM) consists of two core elements, the gas Čerenkov counter, and the Charge Integrator and Encoder (QIE) module. An important function of the BIM is to inhibit triggers when the instantaneous beam intensity exceeds a certain threshold, typically set between approximately 65,000 and 95,000 protons per bucket. To achieve this, the BIM has good time resolution and a linear response over a large dynamic range.

The Cerekov counter is used to measure the beam intensity and a diagram of it is given in Fig. 2.3. The gaseous Cerenkov radiator is an $80 \%$ Argon and $20 \% \mathrm{CO}_{2}$ gas mixture at atmospheric pressure. The baffle is made of a piece of black construction paper and the mirror is a piece of aluminized Kapton set on an elliptical G10 frame. The baffle and the mirror are set at a 45-degree angle, parallel to each other, so that the proton path length between them is independent of the beam position. Once the Čerenkov light is generated, it is reflected by the mirror, passes through the neutral density filters, and then collected by a 8 -stage photomultiplier tube of 2 -inch diameter. This tube is biased at $-870 \mathrm{~V}$ to be operated in the linear dynamic range and to generate appropriate signal amplitude for the QIE module.

The output signal of the photomultiplier tube is then delivered to the QIE module, a NIM 


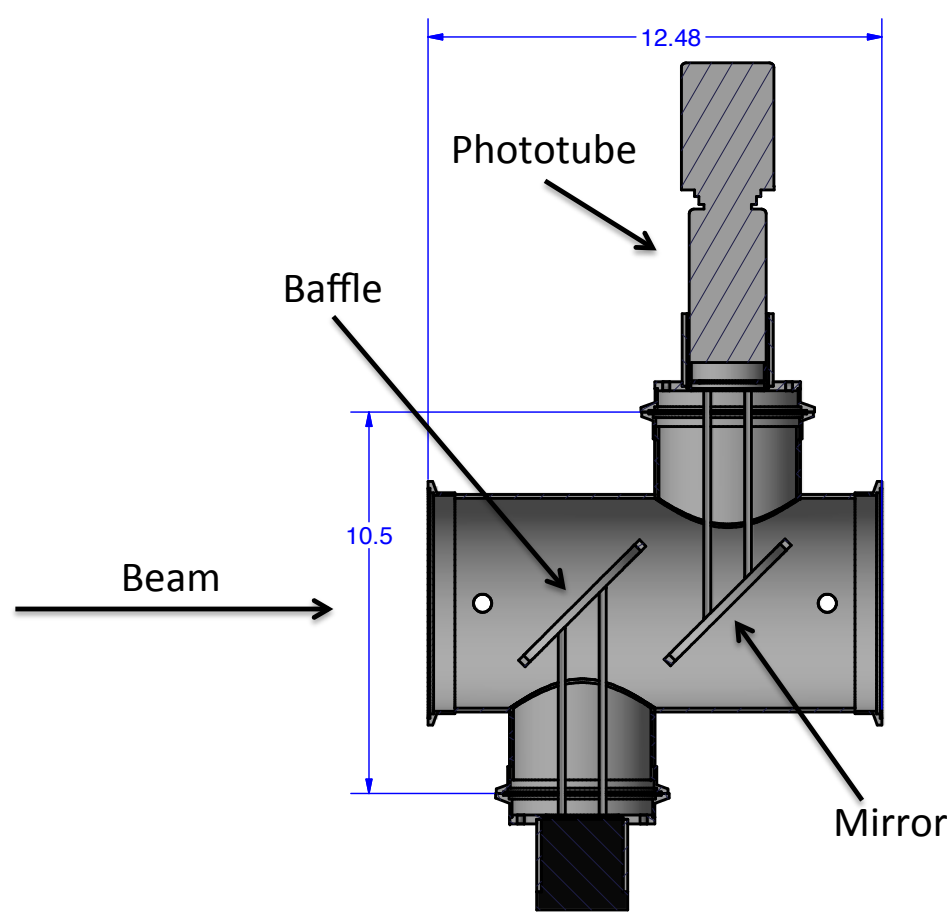

Figure 2.3: The Čerenkov detector of the Beam Intensity Monitor. Dimensions are given in inches.

module that integrates and digitizes the signal by utilizing an integrated circuit chip designed at Fermilab for the CMS experiment. Being synchronized with the Main Injector RF clock, the chip does an ADC conversion on the current input every $18.8 \mathrm{~ns}$, and is capable of providing beam intensity measurement over the range of approximately 30 to more than 3,000,000 protons per bucket with the uncertainty of about $1 \%$.

If the measured intensity of a bucket is greater than the threshold setting, the BIM would inhibit the triggers from not only the threshold-exceeding bucket, but also the 8 buckets before and the 8 buckets after that bucket. In addition to issuing trigger inhibits on high-intensity buckets, the QIE module also provides other important information, which are listed as follows, together with the abbreviations:

- The intensity sum for the whole spill, the $Q I E_{\text {sum }}$.

- The intensity sum when trigger inhibits were issued, the inhibit_block_sum.: 
- The intensity sum during trigger dead time, not including the inhibited buckets while events were being read out, the trigger_sum_no_inhibit.

- A beam-intensity snapshot of buckets neighboring the triggered bucket, including up to 16 buckets before and after the triggered bucket.

- A complete bucket-by-bucket intensity record for the spill.

For example, by using these quantities, together with the readout from G2SEM, we can evaluate the number of the so called "live protons". This quantity corresponds to the exact proton number received by SeaQuest that matches the data taken in each spill and is crucial to all the cross-section measurements. More details about the QIE readouts will be discussed later.

\section{$2.2 \quad$ Targets}

The E906 target system resides in the target cave downstream of the BIM. The layout of the target table is shown in Fig. 2.4. The Z-axis center of the targets is about $129 \mathrm{~cm}$ upstream from the surface of the magnet right after the target system. Seven different targets are used in E906, including two liquid targets, three solid targets, together with an empty flask, and an empty solid target holder (referred to as the "none" target). The empty flask and the none target are used for measurements of background. The configuration of the targets is summarized in Tab. 2.1.

To prepare the liquid targets, gaseous hydrogen and deuterium are liquefied by passing them through the closed-circuit helium refrigeration system. The liquid is then filled into a cylindricallyshaped flask, which is surrounded by an insulating vacuum vessel to minimize the heat load. The flask has hemispherical end-caps and the thickness of the flask wall and the end-cap are $67 \mu \mathrm{m}$ and $51 \mu \mathrm{m}$, respectively. Each of the flasks is capable of holding 2.2 liters of liquid and is high-pressure tested and leak-checked. During operation, the liquid targets are maintained along the vapor-liquid saturation curve. The vapor pressure and the temperature in the flask are monitored constantly. While the pressure measurements are used to determine the liquid density, the temperature readings are used as a cross check and to evaluate the density uncertainty. 


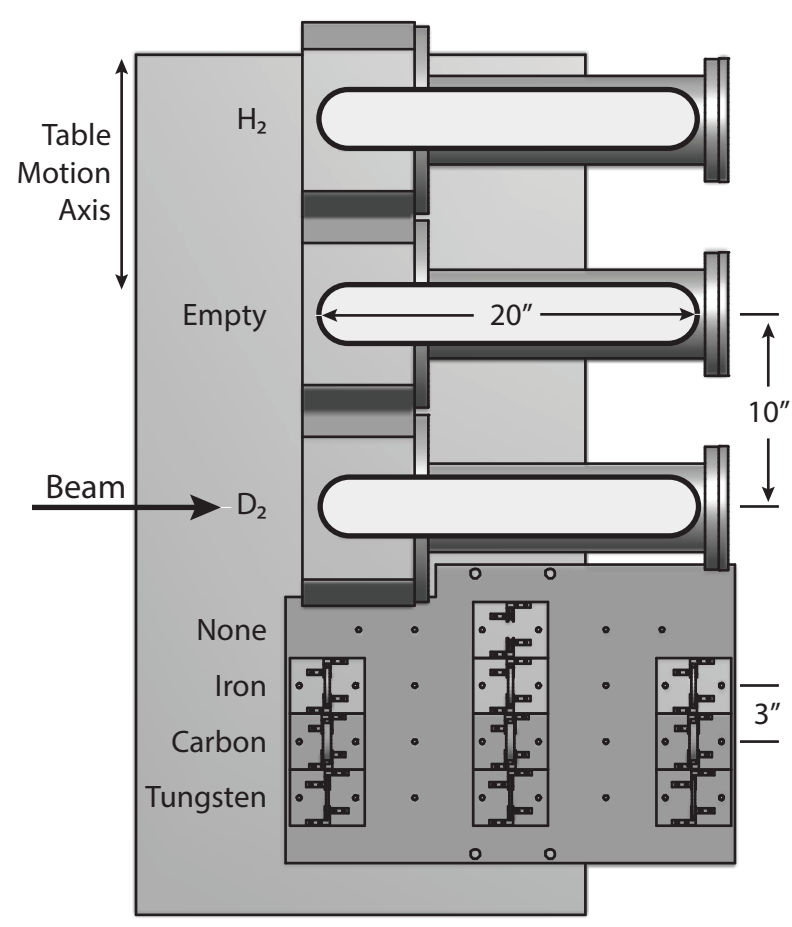

Figure 2.4: The layout of the target table.

\begin{tabular}{c|cccc}
\hline Target Material & Position & Length $(\mathrm{cm})$ & Interaction Length & Spills per Cycle \\
\hline $\mathrm{LH}_{2}$ & 1 & 50.8 & 0.069 & 10 \\
Empty Flask & 2 & - & 0.0016 & 2 \\
$\mathrm{LD}_{2}$ & 3 & 50.8 & 0.12 & 5 \\
None & 4 & - & 0 & 2 \\
$\mathrm{Fe}$ & 5 & 1.905 & 0.114 & 1 \\
$\mathrm{C}$ & 6 & 3.322 & 0.070 & 2 \\
$\mathrm{~W}$ & 7 & 0.953 & 0.096 & 1 \\
\hline
\end{tabular}

Table 2.1: Target configuration.

Each of the solid targets consists of three identically shaped, 2-inch diameter disks. The thickness of each disk is $1 / 3$ of the value listed in Tab. 2.1 and the separation between each plate is $25.4 \mathrm{~cm}$. This arrangement is made so the spatial distribution of the solid targets is more similar to that of the liquid targets and therefore minimize possible spectrometer acceptance variation between targets. One thing to be noticed is that during the Run-II data taking of SeaQuest, the 
spacing between the iron disks was $17.1 \mathrm{~cm}$ only (limited to the platform available at Run-II ), but the effect of this anomaly has been considered negligible.

As can be seen in Fig. 2.4, all the targets are mounted on a table which is movable in the Xaxis direction. With a computer controlled stepper motor driving a lead screw that moves the table on rails, the table moves over a range of about $91.4 \mathrm{~cm}$ and is fast enough to allow the beam-taking target to be changed on a spill by spill basis. During operation, the target positions are verified by magnetic proximity sensors mounted on the table and the platform base, and are recalibrated each time the table passes through the central proximity sensor. The beam-on-target position is also checked by autoradiography and is confirmed to be within $5 \mathrm{~mm}$ of the target center for all targets.

In nominal data taking, target rotation is performed so that the long time-scale systematic effects, like the variation in beam quality or detector acceptance, can be cancelled out in the crosssection ratio measurements. In a cycle of target rotation, the typical number of spills taken by each target is given in Tab. 2.1. This number can be changed based on the different needs of the experiment.

\section{$2.3 \quad$ Magnets}

Two dipole magnets, denoted as FMAG and KMAG, are employed in the SeaQuest spectrometer. The fields generated by these two magnets are set to point in the same vertical direction $(+\mathrm{Y}$ or $-\mathrm{Y})$ in nominal running conditions, and the muons generated are therefore bent horizontally $(+\mathrm{X}$ or $-\mathrm{X})$ by the magnets in the same direction. In this sense, the XZ-plane is also denoted as the "bend plane" while the YZ-plane as the "non-bend plane". This specific two-magnet spectrometer setup, with both magnetic fields pointing in the same direction, is also referred to as a focusing spectrometer.

FMAG, sitting right after the targets, is the magnet used to focus the high-energy muons and keep them in the acceptance of the E906 spectrometer. FMAG is a solid iron magnet composed of $503 \mathrm{~cm} \times 160 \mathrm{~cm} \times 43.2 \mathrm{~cm}$ high-purity iron slabs. The dimensions and some details of the FMAG 

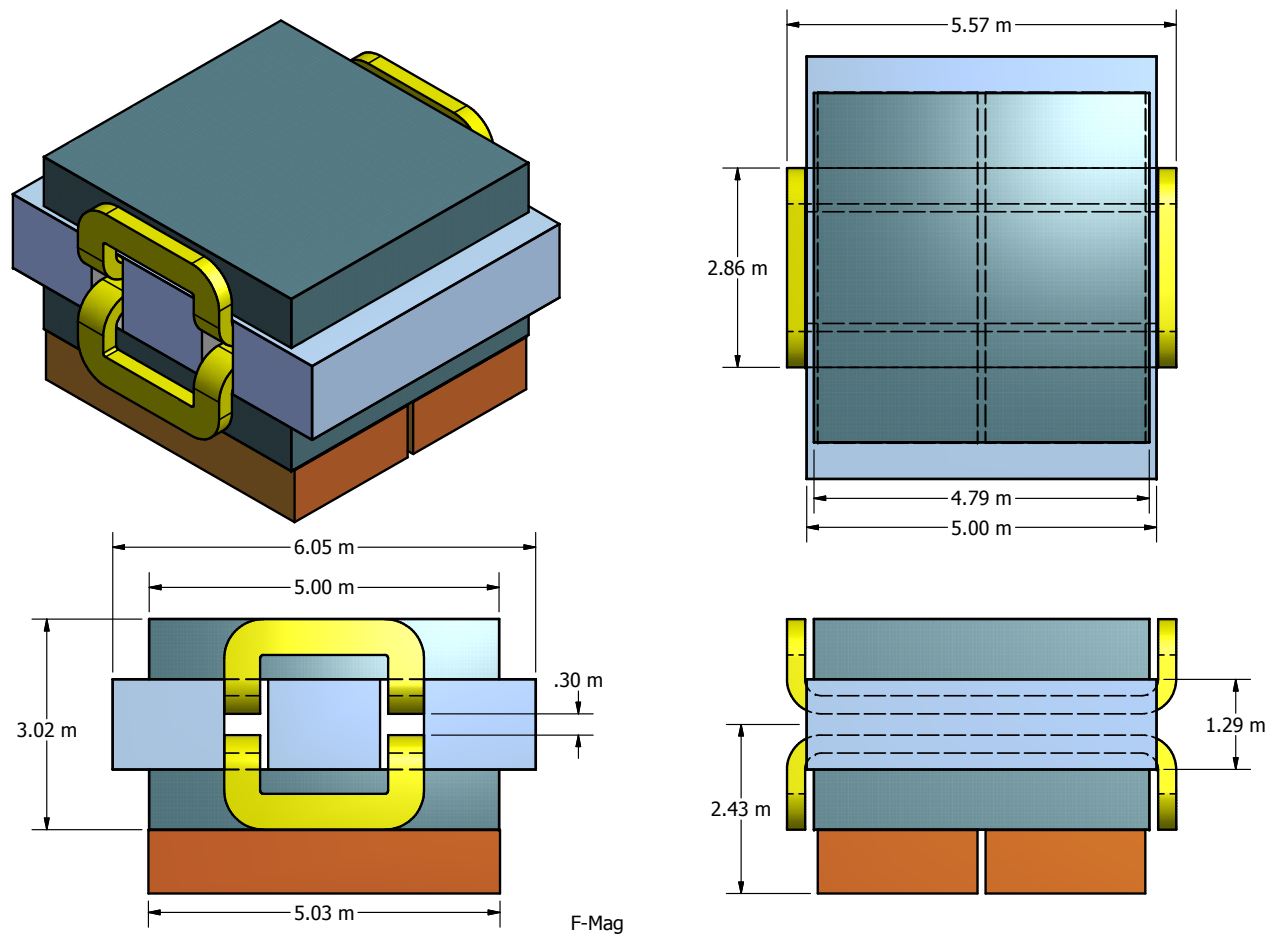

Figure 2.5: The FMAG.

are shown in Fig. 2.5. The aluminum coils of FMAG are one of the three "bedstead" coil sets that belonged to the E866 SM3 magnet. As the coils are excited to the nominal $2000 \mathrm{~A}$ at $25 \mathrm{~V}$, FMAG generates a $1.9 \mathrm{~T}$ central magnetic field and give a total transverse momentum deflection of about 3.07 GeV. A magnetostatic modeling program is used to model the magnetic field distribution inside FMAG, while the final calibration is acquired by examining the reconstructed $J / \psi(3097)$ mass.

A fair number of hadrons are produced as the proton beam passes through the targets and the upstream instrumentation package. To prevent these hadrons, together with the protons that do not interact with the targets, going further into the detector stations, the FMAG also serves as the hadron absorber and the beam dump. A hole of $5 \mathrm{~cm}$ diameter and $25 \mathrm{~cm}$ deep was drilled into the upstream surface of the central iron slab along the beam axis. With this hole, the initial interactions of the remnant protons are further away from the targets and thus lower the possibility 

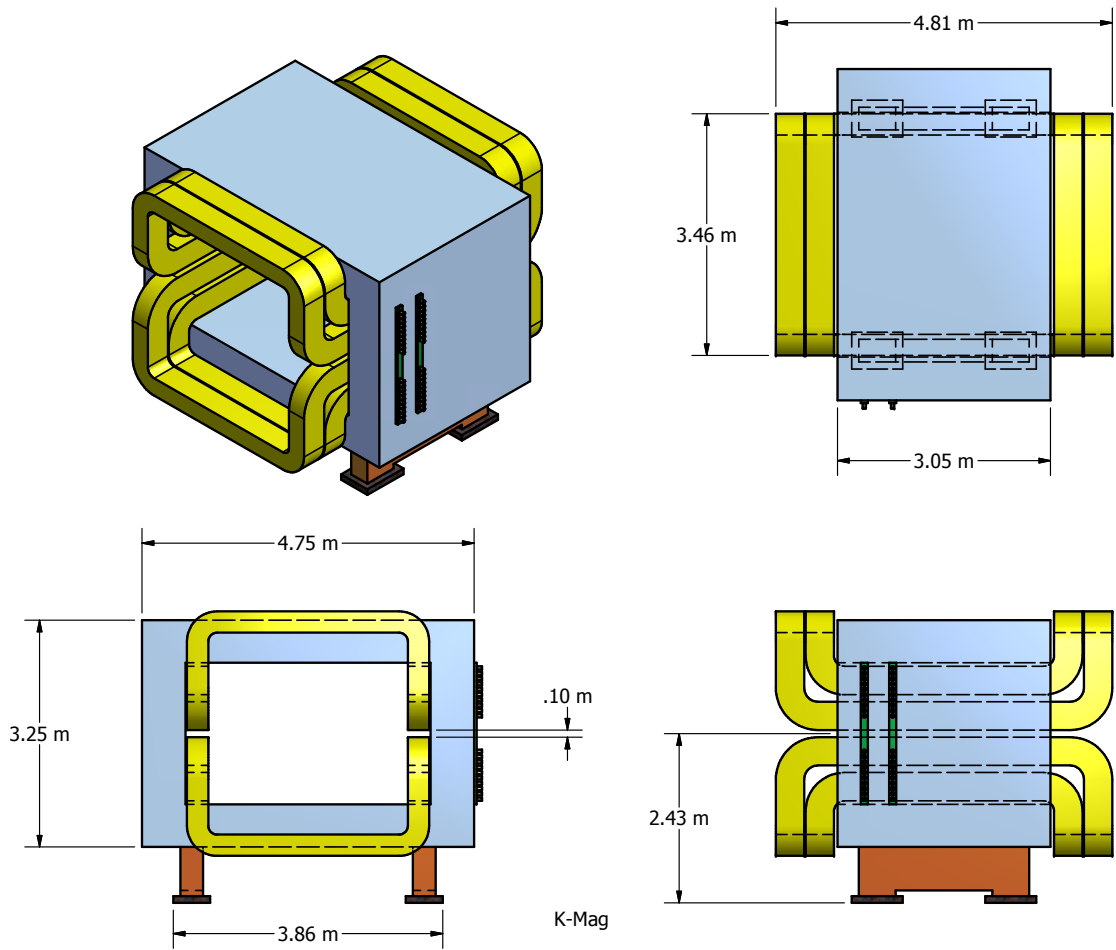

Figure 2.6: The KMAG.

of misidentifying events from the beam dump as the ones from the targets.

The downstream magnet, KMAG, is the magnet that provides the muon momentum measurement. As shown in Fig. 2.6, KMAG is a 3 meter long, rectangular, open-aperture magnet. It was constructed by the E799/KTeV collaboration. Under nominal operation, KMAG would be excited to carry a current of $1600 \mathrm{~A}$ at $270 \mathrm{~V}$ to produce a central magnetic field of $0.4 \mathrm{~T}$, generating a transverse momentum deflection of $0.41 \mathrm{GeV}$. The $\mathrm{KTeV}$ group already measured the magnetic field distribution and the central field calibration was checked by the E906 group with a Hall probe. The final value is again determined by the mass measurement of the $J / \psi(3097)$ resonance. 


\subsection{Tracking Detectors}

\subsubsection{Hodoscopes}

Plastic scintillator hodoscope detectors are used in each of the four tracking stations. The hodoscope planes come in two kinds of spatial arrangement. The one with scintillator paddles oriented vertically, arranged in an array in $\mathrm{X}$ direction, is called the $\mathrm{X}$-plane. The Y-plane is the one with horizontally-oriented paddles arranged in an array in Y direction. The paddles are slightly overlapping with adjacent ones by $0.32 \mathrm{~cm}$ to ensure there is no gap in the acceptance. Although the resolution is quite limited due to the large paddle size, the X-planes are capable of muon X-position measurement and Y-planes can measure the Y-position. Each plane is divided in the middle and is denoted by $\mathrm{T} / \mathrm{B}$ (Top/Bottom) indicating the $+\mathrm{Y} /-\mathrm{Y}$ half for the $\mathrm{X}$-planes, and $\mathrm{L} / \mathrm{R}$ indicating the $+\mathrm{X} /-\mathrm{X}$ half for the Y-planes. Specifications of the hodoscope detectors are given in Tab. 2.2.

Stations 1 and 2 each have an X-plane and a Y-plane. The scintillators and the 1-inch photomultipliers (PMTs) used in these two stations are recycled from the HERMES experiment at DESY. Station 3 has only one X-plane and station 4 has one X-plane and two Y-planes. Station 3 and 4 employ new Eljen EJ-200 scintillators together with the 2-inch PMTs that are either recycled or new Hamamatsu PMTs. The PMTs are powered by LeCroy 1440 High Voltage systems and can be remotely controlled, and the voltage settings are constantly monitored and logged during data taking. Of all the stations, station 4 is the only station that has PMTs mounted on both ends of the scintillator paddles, other stations have just one PMT mounted on the outer end of each paddle. This arrangement is made to reduce the time for the light to travel in the paddle, since the station 4 paddles have significant length.

Although FMAG and KMAG sweep away low energy tracks, a fair number of them still pass through station 1 and station 2 and therefore these stations operate in an environment of significantly higher hit rates than stations 3 and 4 . During the commissioning run in 2012, the hit rates per incident proton recorded by station 1 and 2 were found to be dependent on the 


\begin{tabular}{c|ccccc}
\hline Detector & $\begin{array}{c}\text { Paddle width } \\
(\mathrm{cm})\end{array}$ & $\begin{array}{c}\text { Paddle length } \\
(\mathrm{cm})\end{array}$ & \# of paddles & $\begin{array}{c}\text { Width } \times \text { Height } \\
(\mathrm{cm} \times \mathrm{cm})\end{array}$ & $\begin{array}{c}\text { Z-position } \\
(\mathrm{cm})\end{array}$ \\
\hline H1T & 7.32 & 69.9 & 23 & $162.00 \times 69.85$ & 667.12 \\
H1B & 7.32 & 69.9 & 23 & $162.00 \times 69.85$ & 667.12 \\
H1L & 7.32 & 78.7 & 20 & $78.74 \times 140.12$ & 654.03 \\
H1R & 7.32 & 78.7 & 20 & $78.74 \times 140.12$ & 654.03 \\
H2T & 13.04 & 132 & 16 & $203.24 \times 152.00$ & 1421.06 \\
H2B & 13.04 & 132 & 16 & $203.24 \times 152.00$ & 1421.06 \\
H2L & 13.07 & 152 & 19 & $132.00 \times 241.29$ & 1402.86 \\
H2R & 13.07 & 152 & 19 & $132.00 \times 241.29$ & 1402.86 \\
H3T & 14.59 & 132 & 16 & $227.52 \times 167.64$ & 1958.51 \\
H3B & 14.59 & 132 & 16 & $227.52 \times 167.64$ & 1958.51 \\
H4T & 19.65 & 182.9 & 16 & $304.52 \times 182.88$ & 2234.50 \\
H4B & 19.65 & 182.9 & 16 & $304.52 \times 182.88$ & 2250.68 \\
H4Y1L & 23.48 & 152.4 & 16 & $152.40 \times 365.80$ & 2130.27 \\
H4Y1R & 23.48 & 152.4 & 16 & $152.40 \times 365.80$ & 2146.45 \\
H4Y2L & 23.48 & 152.4 & 16 & $152.40 \times 365.80$ & 2200.44 \\
H4Y2R & 23.48 & 152.4 & 16 & $152.40 \times 365.80$ & 2216.62 \\
\hline
\end{tabular}

Table 2.2: Specifications of the hodoscope planes.

proton beam intensity. This suggested that the phototube voltage dividers of station 1 and 2 were "sagging" and could not maintain the voltage applied to the dynodes at high beam intensity. A new MOSFET-stabilized PMT base was subsequently designed, installed, and successfully resolved this issue.

As charged tracks pass through the hodoscopes, PMTs collect the light signals generated in the scintillators and produce analog pulse outputs. The width of the pulses is reduced by "clip lines" attached to the PMT bases to a full width of about 10-15 ns. These output pulses are then processed through CAMAC discriminators, digitized by Time-to-Digital Converters (TDCs), and finally transmitted to the NIM- and FPGA-based trigger system.

\subsubsection{Drift Chambers}

Except station 4, drift chambers are installed in the other 3 stations to precisely measure the $\mathrm{x}-\mathrm{y}$ position of the particle tracks as they pass through different chamber planes. While station 1 


\begin{tabular}{ll|cccc}
\hline Chamber & Plane & \# of wires & Cell width $(\mathrm{cm})$ & Width $\times$ Height $(\mathrm{cm})$ & Z-position $(\mathrm{cm})$ \\
\hline D1.1 & X & 160 & 0.64 & $102 \times 122$ & 617 \\
& U, V & 201 & 0.64 & $101 \times 122$ & \pm 20 \\
D1.2 & X & 320 & 0.50 & $153 \times 137$ & - \\
& U, V & 384 & 0.50 & $153 \times 137$ & \pm 1.2 \\
D2 & X & 112 & 2.1 & $233 \times 264$ & 1347 \\
& U, V & 128 & 2.0 & $233 \times 264$ & \pm 25 \\
D3p & X & 116 & 2.0 & $232 \times 166$ & 1931 \\
& U, V & 134 & 2.0 & $268 \times 166$ & \pm 6 \\
D3m.1 & X & 176 & 1.0 & $179 \times 168$ & 1879 \\
& U, V & 208 & 1.0 & $171 \times 163$ & \pm 19 \\
D3m.2 & X & 116 & 2.0 & $232 \times 166$ & 1895 \\
& U, V & 134 & 2.0 & $268 \times 166$ & \pm 6 \\
\hline
\end{tabular}

Table 2.3: Specifications of the wire chambers. The Z-position of $\mathrm{U}$ and $\mathrm{V}$ planes are relative to the associated X planes. The Z-position of D1.1 and D1.2 were changed for few times after the installation and commissioning of D1.2. Since the data analyzed in this thesis was collected without D1.2, the numbers given are the ones before the installation of D1.2

and station 2 each employ one drift chamber, which are called D1 and D2, two drift chambers are used in station 3 to provide adequate acceptance coverage. These two chambers are denoted as D3p and D3m, where "p" and "m" indicates "plus" and "minus", as D3p covers the upper $(+\mathrm{Y})$ half measurement and D3m the lower (-Y) half.

A total of six wire planes are in each of the drift chambers, and all the planes are parallel to the XY-plane. The wire planes can be categorized into three different "views" depending on the wire orientation. The $\mathrm{X}$-view planes have vertical wires and the $\mathrm{U}$ - and $\mathrm{V}$-planes have ones that are titled away from the Y-axis by $14^{\circ}$ and $-14^{\circ}$ respectively. The "primed" planes of each view are right next to the "unprimed" planes and are transversely shifted by half of the drift cell width. This two-plane setup in each view can help to solve the so-called "left-right ambiguity", which is related to the fact that one cannot tell on which side of the fired wire the track passed by. Some details on the chambers are summarized in Tab. 2.3.

With the progress of the experiment, upgraded versions of D1 and D3m have been constructed. The new chambers are wider than the old ones, and therefore have increased acceptance 


\begin{tabular}{lllcc}
\hline Run & Dates & St. 1 & St. 2 & St. 3 \\
\hline I & 2012 Mar. - 2012 Apr. & D1.1 & D2 & D3p \& D3m.1 \\
II & 2013 Nov. - 2014 Aug. & D1.1 & D2 & D3p \& D3m.2 \\
III & 2014 Nov. - 2015 Jul. & D1.1 & D2 & D3p \& D3m.2 \\
IV & 2015 Nov. - 2016 Mar. & D1.2 & D2 & D3p \& D3m.2 \\
IV & 2016 Mar. - 2016 Jul. & D1.1 \& D1.2 & D2 & D3p \& D3m.2 \\
V & 2016 Nov. - 2017 Jul. & D1.1 \& D1.2 & D2 & D3p \& D3m.2 \\
\hline
\end{tabular}

Table 2.4: Chamber combination of different run periods.

in the kinematic region of high $x_{2}$ as desired by SeaQuest. Different versions of D1 and D3m are further labeled by a version number after the decimal point, like D3m.1 and D3m.2. A list of the individual set of chambers used in different data-taking periods is given in Tab. 2.4. The D1.1 chamber was originally used in E866/NuSea while D2 and D3m.1 were inherited from E605. These chambers had been in storage for years before SeaQuest. To bring these chambers back to working condition, a fair effort was made, including restringing wires for the broken or loose ones and implementing new readout electronics. D1.2, D3p, and D3m.2 are new chambers that were specifically designed and built for SeaQuest. The collaborators from Tokyo-Tech took charge of the fabrication of D3p and D3m.2, and D1.2 was constructed by the University of Colorado group. More details related to D1.2 are given in Appendix A.

Other than D1.2, a gas mixture of Argon:Methane:CF4 (88\%:8\%:4\%) is used for all the chambers. Since the hit-rate of station 1 is higher than the other stations, the ion drift velocity of the gas used in D1 should be higher so the chamber can have a faster response and better performance. Initially, the gas mixture of Argon:Isobutane:CF4:Methylal (68\%:13\%:16\%:3\%), which has a drift velocity greater than $50 \mu \mathrm{m} / \mathrm{ns}$, was proposed, but was later modified to the mixture of Argon:Isobutane:CF4:Methylal (81\%:12\%:5\%:2\%) to have a better gas gain. This new mixture is only used in D1.2 because of safety issues; it is a flammable gas and D1.1 is quite leaky.

The high voltages of the chambers are supplied by different NIM high-voltage modules set up in the control room. The voltages were set at the edge of the efficiency plateau so the chambers 
can work at adequate efficiency with the lowest possible voltage to minimized the chance of damage caused by sparking in the chamber. Like the high voltages supplied to the PMTs, the voltage and current outputs of the modules are continuously monitored and can be traced back if desired. The nominal setting of the high voltages is logged.

The raw analog signals from the sense wires of the chambers are first processed by the amplifier card called "ASDQ" (acronym of Amplification, Shaper, Discriminator, Charge integration), which was originally designed at the University of Pennsylvania for the CDF experiment [102]. The ASDQ cards convert the charge inputs into voltage signals, perform amplification, signal shaping, discrimination on these signals, and then feed the differential outputs to the "Level Shifter Boards" (LSBs). The LSBs are responsible for supplying voltage to the ASDQ cards and be converting the signals received into LVDS signals. These LVDS outputs are transmitted to the TDC modules to be digitized and then collected by the DAQ.

The performance of the chambers has been studied using the data collected from Apr. 2014 to Jun. 2015. All the chamber planes have detection efficiency greater than $95 \%$ with $70 \%$ of the planes being nearly 100\% efficient. Based on the chamber efficiency, the track reconstruction efficiency is greater than $90 \%$. The average position resolution of the chambers is better than 400 $\mu \mathrm{m}$, which corresponds to a momentum resolution of $\Delta p / p(\%)=0.03 \cdot p(\mathrm{GeV} / c)$. The contribution to the total mass resolution from the position resolution is therefore expected to be less than $10 \%$.

\subsubsection{Proportional Tubes}

Before entering station 4, the particles went through a $1 \mathrm{~m}$ thick iron wall. The hadrons are absorbed in the iron wall, and electrons would shower and are scattered more significantly than muons. Therefore, a small deflection of the track after passing through the iron wall is the key feature to be used for muon identification.

To precisely determine the track position, the tracking detectors used in station 4 are composed of proportional tubes. The layout of the station 4 proportional tubes is given in Fig. 2.7. There are four proportional tube planes with proportional tubes oriented in the horizontal or ver- 

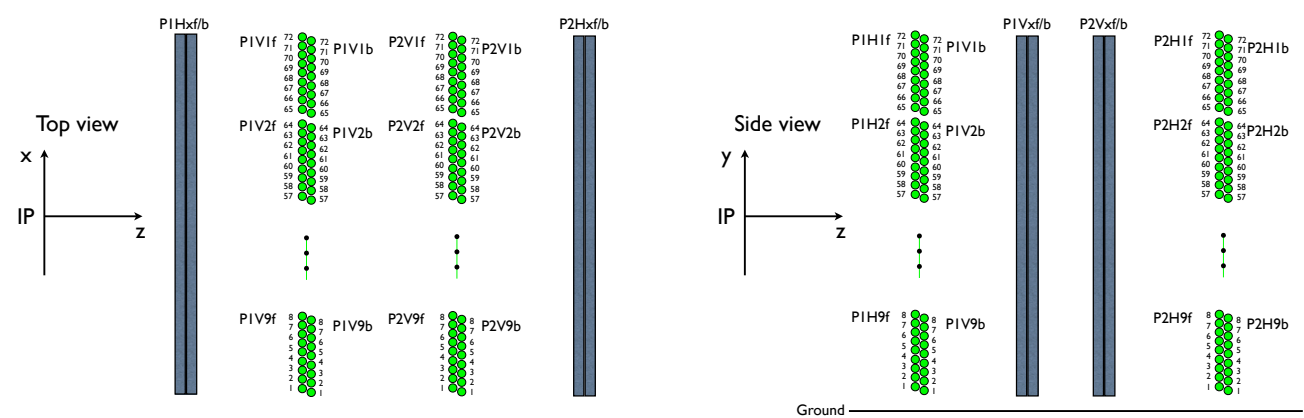

Figure 2.7: Left: Top (XZ-) view of the proportional tubes. Right: Side (YZ-) view of the proportional tubes.

tical direction. Similar to the hodoscopes, the horizontal proportional tubes in the first and fourth planes measure the Y-coordinates of the tracks, and the vertical tubes in the second and third planes are in charge of the $\mathrm{X}$-coordinate measurements.

Originally, the proportional tube modules were developed for a Homeland Security project at Los Alamos National Laboratory [103]. Every plane consists of 9 proportional tube modules that includes 16 proportional tubes in each. These 16 proportional tubes are further divided into two staggered sub-planes, as can be seen in Fig. 2.7. Like the primed-unprimed planes in each view of the drift chambers, this paired-plane setup helps to resolve the left-right ambiguity. The proportional tubes are made by 2 -inch diameter aluminum tubes of $1 / 16$ inch wall thickness. The sense wires strung at the center of the tubes are gold-plated $20 \mu \mathrm{m}$ tungsten wires, which are typically set to $1800 \mathrm{~V}$. The gas used in the proportional tubes is the same Argon:Methane:CF4 (88\%:8\%:4\%) mixture used for most of the chambers.

The Nanometric Systems N-277 16-channel Amplifier/Discriminator cards are utilized to process signals from groups of 16 proportional tubes. The maximum drift time is $650 \mathrm{~ns}$, which gives a hit-rate tolerance of up to $2 \mathrm{MHz}$ for a single wire, while the hit rate is in general lower than $1 \mathrm{MHz}$ per wire. The position resolution was found to be $500 \mu \mathrm{m}$, which suffices for the purpose of muon identification. 


\begin{tabular}{cccc}
\hline Plane & $\begin{array}{c}\text { Width } \times \text { Height } \\
(\mathrm{cm} \times \mathrm{cm})\end{array}$ & $\begin{array}{c}\text { Forward sub-plane Z-position } \\
(\mathrm{cm})\end{array}$ & $\begin{array}{c}\text { Backward sub-plane Z-position } \\
(\mathrm{cm})\end{array}$ \\
\hline P1H & $368.3 \times 388.6$ & 2099 & 2103 \\
P1V & $388.6 \times 368.3$ & 2175 & 2179 \\
P2H & $368.3 \times 388.6$ & 2389 & 2393 \\
P2V & $388.6 \times 368.3$ & 2367 & 2371 \\
\hline
\end{tabular}

Table 2.5: Specifications of the proportional tube planes.

\subsection{Trigger}

The signals from the hodoscopes are used as inputs for the SeaQuest trigger. The trigger is optimized for high mass dimuons generated from the targets, and in the meantime suppresses events from other sources like charmonium decays to keep the trigger rate low enough to maintain an acceptable DAQ deadtime. Two types of trigger systems are utilized, the NIM-based trigger and the FPGA-based trigger.

The NIM-based trigger is a simpler trigger that employs Nuclear Instrumentation Modules (NIM) for trigger formation. Under normal physics data taking conditions, the two so called NIM-1 and NIM-3 triggers are used. The NIM-1 trigger is the coincidence of signals from the top-half Y-measuring hodoscopes of all stations, or the coincidence of signals from the bottom-half Y-measuring hodoscopes. This trigger is useful for some quick preliminary efficiency studies. The NIM-3 trigger is a random trigger formed by the coincidence of $7.5 \mathrm{kHz}$ pulses generated by a gate generator and the RF signals from the Fermilab accelerator division. The data collected by this random trigger is used for background studies.

The FPGA-based trigger system consists of 9 CAEN V1495 VME modules, and an Altera EPIC20F400C6 FPGA (Field Programmable Gate Array) is incorporated on the V1495 modules. The trigger is divided into three different "Levels" of V1495 modules, from Level-0 to Level-2. At the first stage, the outputs from the hodoscope planes are categorized into four different "quadrants": the top and the bottom halves of the X-planes, and those two halves of the Y-planes. Signals from 
each of the quadrants is processed by an individual Level-0 V1495 module. The Level-0 module can be operated in two modes, the "Pulser" mode and the "Production" mode. In "Pulser" mode, designated hit patterns can be generated as output and can be used to examine the behavior of the Level-1 and Level-2 trigger. For nominal physics runs, the Level-0 module is set to the "Production" mode, under which the input signals are delivered to the Level-1 modules directly.

Another four V1495 modules are used for the Level-1. While each of them handles input from one Level-0 board, only the two Level-0 modules for the X-planes are used for the Level-1 trigger determination. The Level-1 trigger acts as a track finder, which is responsible for identifying the passage of candidate muons by comparing the hits on the hodoscope planes with a list of preprogrammed hit patterns. These patterns are called "trigger roads", and a specific set of trigger roads is designated as a particular "Roadset". Initially, the Roadset was determined based on Monte Carlo simulations. The Roadset was then iteratively determined from data: a loop of implementing into the firmware of the trigger, being tested, and modified so that it can be as effective as possible. An ever-increasing ID index was given to a modified Roadset and Roadset 57 was the first successful Roadset SeaQuest used. Depending on the need of the experiment, the Roadset in use can be changed quickly and easily.

Once the hit patterns are found to match the trigger roads by Level-1, a bit string, which is binned by charge and average transverse (X-direction) momentum $p_{T}$, is generated and sent to the Level-2 trigger. The Level-2 trigger is a single V1495 module which acts as a track correlator. The roads found by Level-1 are combined to form all possible pairs, and then checked against pre-programmed lookup tables to see if they are valid. Five different Level-2 trigger requirements are adopted and the definitions of them are given in Tab 2.6. The FPGA-1 is the main trigger for physics data, which requires two oppositely charged tracks, one from the top side and the other from the bottom side. The FPGA-3 trigger is useful for background estimations that will be discussed more in the data analysis section. These five Level-2 triggers are sent out individually to the "Trigger Supervisor", a VME module developed at the Thomas Jefferson National Accelerator Facility (JLab) [104], which will be described in more detail in the next section. The trigger 


\begin{tabular}{llllll}
\hline Name & Side & Charge & $p_{T}$ cut & Prescale factor & Description \\
\hline FPGA-1 & TB/BT & $+-/-+$ & - & 1 & Main physics trigger \\
FPGA-2 & TT/BB & $+-/-+$ & - & 10000 & Same-side trigger \\
FPGA-3 & TB/BT & $++/-$ & - & 123 & Like-Charge trigger \\
FPGA-4 & T/B & $+/-$ & - & 25461 & All singles trigger \\
FPGA-5 & T/B & $+/-$ & $p_{T}>3 \mathrm{GeV}$ & 2427 & High- $p_{T}$ singles trigger \\
\hline
\end{tabular}

Table 2.6: Definitions of Level-2 triggers. The T or B used in the "Side" column denotes either the top side or the bottom side from which the tracks are found.

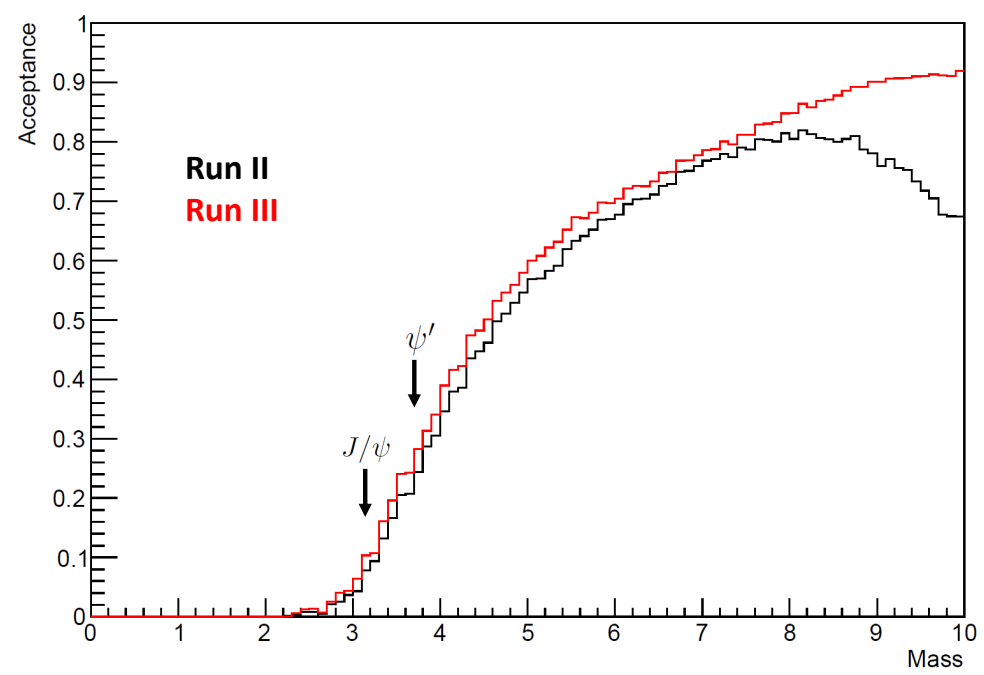

Figure 2.8: Trigger acceptance versus mass for Run-II and Run-III FPGA-1 Roadsets [105]. The masses of $J / \psi(3097)$ and $\psi^{\prime}(3686)$ resonances are indicated.

acceptance versus dimuon mass, relative to the geometric acceptance of the hodoscopes, is given in Fig. 2.8 for both the Run-II and Run-III FPGA-1 roadsets. As shown in the plot, the events with mass smaller than $4 \mathrm{GeV}$ are strongly suppressed to exclude the dimuons from charmonium decay, and the high mass dimuons, which are dominated by the Drell-Yan process, have a much larger acceptance.

\subsection{Data Acquisition and Handling}

Based on the amount of data transferred and the relative timing issue, the data acquisition (DAQ) system of SeaQuest is divided into sub-systems labeled as the "Main DAQ", "Scaler 
DAQ", and "Beam DAQ", together with a set of auxiliary scripts denoted as "Slow Controls". Both the Main DAQ and Scaler DAQ employ the VME-based "CODA" (CEBAF On-line Data Acquisition) [106] system, which was developed by the data acquisition group at JLab.

\subsubsection{Main DAQ}

On an event-by-event basis, the signals coming from the SeaQuest spectrometer are processed and recorded by the Main DAQ. A Scientific Linux PC has been utilized to run the CODA software that collects data from its various components. The most upstream elements of the Main DAQ system are the front-end VME crates. Each of the VME crates is responsible for handling the output signals from a specific part of the spectrometer. While the number of VME crates can vary according to the spectrometer configuration, there are in general about 14 crates along with one crate for the Trigger Supervisor (TS). Each VME crate has a single board CPU denoted as the Read Out Controller (ROC), a Trigger Interface (TI) card, and a number of TDCs receiving detector signals.

Once an FPGA Level-2 or NIM trigger is issued and not being vetoed by the QIE module, it is accepted by the TS, which is capable of receiving 12 separate trigger inputs. Five of these input channels are reserved for FPGA triggers, four for NIM triggers, and the remaining three are for the "flush" events, the beginning of spill (BOS) and end of spill (EOS) signals. Eight TS input channels are pre-scalable and the prescale factor $F_{p s}$ for each of the FPGA triggers is listed in Tab. 2.6. With prescaling, only 1 out of every $F_{p s}$ triggers received would be recognized by the TS. Other than the FPGA-1 trigger, the FPGA trigger rates are scaled down by their prescale factor to approximately less than $10 \%$ of the FPGA-1 trigger rate. In this way, the data taking of the

physics trigger is less affected. The subsequent workflow of the Main DAQ is illustrated in Fig. 2.9 and the details of each numbered step is described as follows:

(1) The Trigger Supervisor (TS) receives the trigger from the V1495 Level-2 or NIM modules, and TS is set to busy. 


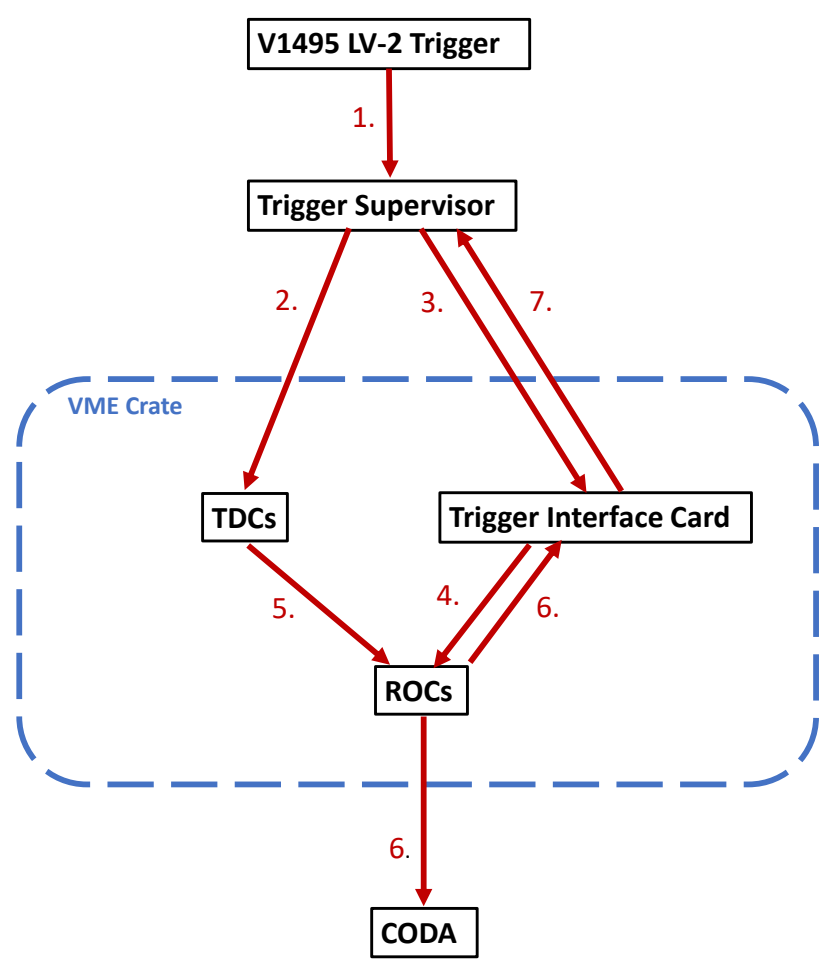

Figure 2.9: The workflow of the Main DAQ.

(2) Level-1 accept is fanned out to all TDCs. TDCs then stop data taking and save all hits in their ring buffers.

(3) After being delayed by $32 \mu \mathrm{s}$, the trigger is sent to all TI cards. This is the so-called copy-in-progress time.

(4) After another $10 \mu \mathrm{s}$, each TI instructs the ROC to read out the TDCs.

(5) The TDCs deliver the hit information to the ROC through the VME backplane. This process takes about $100 \mu \mathrm{s}$.

(6) The ROC tells the TI that the reading process is done, and the collected data is sent to CODA via private network.

(7) An acknowledgement signal (ACK) is issued by the TI back to the TS, indicating that this 
VME readout is done. After receiving ACKs from all VME crates, the TS is reset and is ready to take the next trigger.

Since the Fall of 2016, an improved readout scheme has been implemented in which the data is stored locally in the TDC modules during a spill and then transferred through the VME backplanes between spills. This successfully reduced the readout time from $\approx 150 \mu \mathrm{s}$ to $\approx 30 \mu \mathrm{s}$. Other than the steps given above, a trigger is also sent to the QIE module by the TS so that the beam intensity in a 12- to 16-bucket time window around the triggering bucket is measured.

\subsubsection{Scaler DAQ}

The Scaler DAQ collects scaler data from the beam, detectors, and trigger, and provides useful information for monitoring and diagnostic purposes. Controlled by a standalone CODA system running on a separate PC, the Scaler DAQ is totally independent of the Main DAQ. One VME crate is utilized in the Scaler DAQ system, with one ROC CPU board and four scaler cards installed.

One of the four VME scalers is triggered by the coincidence of signals from the beam spill gate and a $7.5 \mathrm{kHz}$ gate generator. The hits from two unrelated hodoscopes are recorded by this scaler at $7.5 \mathrm{kHz}$, with which SeaQuest can directly observe beam fluctuations on this time scale. The other three scalers are enabled to count by the BOS signal, stopped and read out with the EOS signal, and therefore record the integrated counts over a spill. The data collected by these spill-level scalers includes various Main DAQ trigger rates, the beam intensity recorded by the upstream instrumentation package, and the hit rates of hodoscope arrays. The Scaler DAQ data is processed by the analysis program which generates realtime outputs, such as the fast Fourier transform of the beam intensity, that are not only monitored by SeaQuest, but also by the Fermilab Accelerator Division. 


\subsubsection{Beam DAQ}

The Beam DAQ is responsible for reading the data from the Čerenkov beam intensity monitor, with which the $53 \mathrm{MHz}$ beam structure is measured. Together with the recorded data listed in Section 2.1.1, the QIE module also provides the bucket-by-bucket sum of beam intensity $I$, and the sum of the bucket-intensity squared $I^{2}$ for every spill. These values are used to calculate the 53 $\mathrm{MHz}$ duty factor, $D F$, that is defined as

$$
D F=\frac{\left(\sum I_{i} / N\right)^{2}}{\left(\sum I_{i}^{2}\right) / N}=\frac{\langle I\rangle^{2}}{\left\langle I^{2}\right\rangle},
$$

where $N$ denotes the number of buckets in a spill. This duty factor is the primary reference used by the Accelerator Division for tuning the beam delivered to SeaQuest.

The control and readout of the QIE module is carried out by the custom DAQ program that communicates with the QIE through a 100 Mbps Ethernet interface. This Ethernet interface together with two additional ones are used for fast enough data output and handling between the spills. The data produced during a spill is stored in the QIE board and is read out once the EOS signal is received. Due to the fact that the accumulated data is approximately $300 \mathrm{MB}$, a multithreading treatment was utilized in order to have the data processed before the next spill arrives. While three threads are employed for transferring data, up to eight threads are utilized for the data analysis. The analyzed results can be accessed via a public webpage for realtime beam-quality monitoring.

\subsubsection{Slow Controls}

The slow control system consists of scripts designed to help synchronize the DAQ data stream, to retrieve and store data of per-spill frequency or slower, and to monitor miscellaneous process variables (PVs) and the status of the experiment. The Experimental Physics and Industrial Control System (EPICS) [107] is utilized by the slow control scripts mainly as a medium for data broadcasting over the network.

An unique ID number is assigned to each spill as the primary reference so that the data 
recorded by different independent DAQ systems can be synchronized. This ID is denoted as the spillID and a master spillID is stored in a file on the SeaQuest RAID array. This file is updated with the spillID once a EOS signal arrives, and is then inserted into the CODA output files of both the Main DAQ and Scaler DAQ. The master spillID is also written into the memory of EPICS server to be accessed by the realtime analysis programs.

Triggered by the EOS signal, the slow control system collects data including:

- The high voltage setting of the chambers and proportional tubes.

- The beam parameters via Fermilab's accelerator controls network (ACNET), such as the accelerator configuration, delivered beam quality, and the status of the magnets.:

- The target PVs via EPICs server, such as the target-rotation pattern, the temperature, and the pressure of the cryogenics.

- Environmental conditions, such as the temperature, pressure, and humidity of the spectrometer hall.

The data recorded is stored as text files, and also inserted into the CODA output of the Main and Scaler DAQ.

Various realtime checks are also performed by the monitoring scripts, such as the condition of the targets, the data-updating progress and the disk space available, and the running status of the DAQ system. Once a critical issue is found, the personnel on shift receives alarm and the experts are notified by email or text message.

\subsubsection{Data Decoding and Storage}

In general, one run of physics data taking takes about an hour and corresponds to approximately 1 GB of raw data collected by the Main DAQ. The Main-DAQ CODA file, together with the data produced by other DAQ subsystems, are stored on the SeaQuest servers and backed up by the tape storage service managed by the Fermilab Computing Division. These raw data files are 

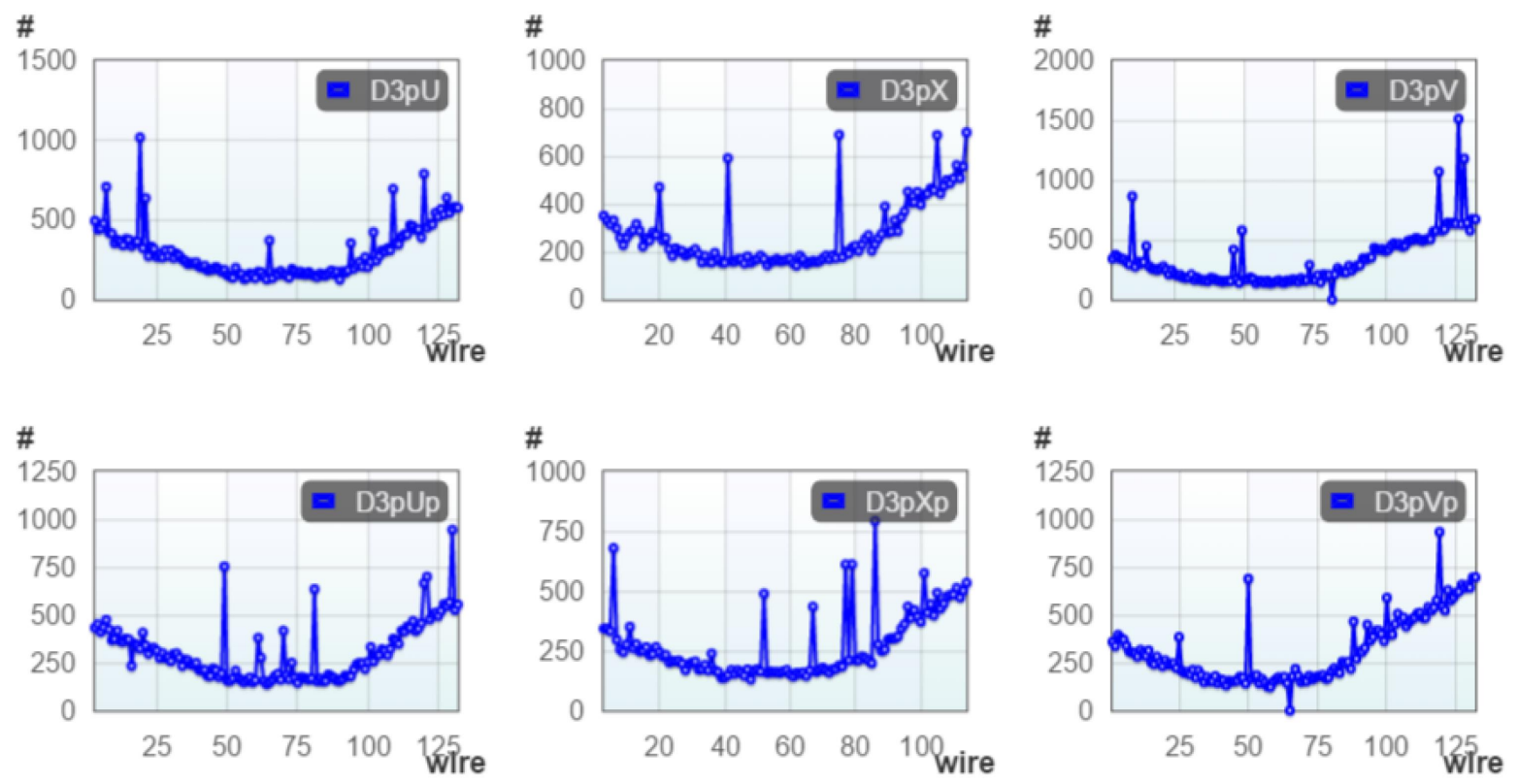

Figure 2.10: An example of D3p hit distributions. Some hot channels and few deal channels can be observed.

processed by the "decoder" program which decodes the data from its original format, manages the data with some additional information and calculations, and then filled the extracted information into the SeaQuest MySQL database. For each run, a corresponding MySQL scheme is designated, in which the data is stored as well-organized tables. The data is duplicated across multiple MySQL servers and can be flexibly retrieved for analysis.

One important feature of the decoder is that the decoding is performed in a "sampling mode", which enables the decoder to provide quick results for "SeaScape", a web-based tool that analyzes collected data and produces graphical outputs. SeaScape provides easy access to the DAQ readouts and facilitates monitoring of experiment. An example plot of the drift-chamber hit distribution given by SeaScape is shown in Fig. 2.10. 


\section{Chapter 3}

\section{Data Analysis}

\subsection{Data Sets}

\begin{tabular}{ccl}
\hline Roadset & \# of roads & Description \\
\hline 57 & 871 & First Roadset for physics analysis. \\
59 & 891 & Few dark-photon-search roads added. \\
62 & 891 & Add more dark-photon-search roads. Some hot roads removed. \\
67 & 977 & Roads for flipped magnetic field. Re-evaluated the hot roads. \\
\hline
\end{tabular}

Table 3.1: Description of Roadset 57, 59, 62, and 67.

The data sets used in this analysis include Roadset 57, 59, 62, and 67. Some description and quantities for these Roadsets can be found in Tab. 3.1 and 3.2. Roadset 57 is quite similar to Roadset 59, which has a few roads dedicated to the dark-photon search. Since the data in these two Roadsets were all taken in the Run-II period in 2014 before the Fermilab accelerator shutdown, it is decided to combine data of these two Roadsets and have it denoted as the Run-II data. Due to various reasons listed in Tab. 3.3 , some data are excluded from the analysis at this moment. Various efforts are underway to rescue as much of this data as possible by the SeaQuest collaboration.

\subsection{Track Reconstruction}

The main challenge with track reconstruction for SeaQuest is the multiple scattering of tracks as they travel through the 5-meter long FMAG. The SeaQuest collaboration aims to have two independent track reconstruction programs developed. By comparing the tracks produced by these 


\begin{tabular}{cclc}
\hline Run period & Roadset & Date & \# of data runs \\
\hline II & 57 & Jun. 25 2014 - Aug. 20 2014 & 1304 \\
II & 59 & Aug. 20 2014 - Sep. 03 2014 & 467 \\
III & 62 & Nov. 08 2014 - Jan. 14 2015 & 1253 \\
III & 67 & Jan. 25 2015 - Jun. 19 2015 & 3096 \\
\hline
\end{tabular}

Table 3.2: Summary of Roadset 57, 59, 62 , and 67 data.

\begin{tabular}{cll}
\hline Roadset & Range of spills & Note \\
\hline 57 & $303215-310954$ & Missing QIE values \\
59 & $371870-376533$ & Trigger timing shift \\
59 & $378366-379333$ & Trigger timing shift \\
62 & $394287-409540$ & Commissioning period of Run-III \\
62 & $416207-424180$ & Manual target control \\
62 & $482574-484924$ & Magnetic field flipped before changing Roadset \\
67 & $526201-526364$ & Bad QIE inhibit timing \\
67 & $581369-582460$ & KMAG off \\
\hline
\end{tabular}

Table 3.3: Ranges of spills that are excluded from the analysis.

tracking programs, some underlying issues can be found and more reliable tracking results can thus be acquired.

The main track reconstruction program of SeaQuest at this point is denoted as the "kTracker", where the "k" stands for the Kalman-Filter method [108] applied in determining the dimuon-vertex. This program was developed mainly by Kun Liu, a collaborator from the Los Alamos National Laboratory. The whole track reconstruction process can be divided into a few stages as the pre-tracking analysis, track reconstruction, and vertex finding. The working principle and the workflow of the kTracker, as described in [109, is briefly explained in this section.

\subsubsection{Pre-tracking analysis}

In order to minimize the work load and enhance the performance of the time-consuming tracking process, a few methods are adopted to reasonably remove potential noise hits before passing the event to the tracker. The two approaches that are applied to all detector hits are: 
- Exclude out-of-time hits: Discard detector hits with TDC time falling out of the predefined TDC time window. This is used to remove random noise, and signals from unwanted sources like cosmic rays.

- Remove after-pulses: In an event, only the first pulse of each channel is accepted as a valid signal. The following pulses are discarded. This is used to remove redundant hits, which might come from the echo of signals in the same channel.

For the signals coming from the drift chambers, an additional step is applied for cases involving groups of neighboring fired wires which are called "hit clusters". The hit clusters are categorized into three categories based on the way they are formed, and are thus treated differently. The first category is related to $\delta$ rays, which are electrons of relatively high energy that can be produced by nearly head-on collisions in the passage of a primary ionizing particle. A delta ray is capable of producing secondary ionization. If a cluster consists of more than two contiguous hits and the average of their TDC-time difference is large, this cluster would be considered to be generated by $\delta$-rays propagating in the $\mathrm{X}$-Y plane. In this case, since it is desired to keep the hit possibly induced by the muon track, the two hits on the edges of the cluster are kept and the middle ones are rejected. The second category corresponds to the so-called "cell-edge" hits. As a track passes through a trajectory close to the center of two adjacent wires, it may induce ionizations that results in both wires fired and causes the corresponding hits have long drift distance close to half of the cell width. For this type of hit pairs, the hit with longer drift distance is discarded. The last category is from electronic noise. If the average TDC time difference of more than two contiguous hits on adjacent wires are found to be less than $10 \mathrm{~ns}$, these hits are all regarded as coming from electronic noise and thus removed.

After the hit-reduction process, a requirement on upper limits of the number of detector hits is applied. As listed in Tab. 3.4, these so-called "multiplicity cuts" are used to discard events having too many registered hits that would cause the tracking program to take significant time evaluating all possible hit combinations with questionable tracking results, and therefore are impractical for 


\begin{tabular}{lc}
\hline Detector & Limit of number of hits \\
\hline D1 & 300 \\
D2 & 200 \\
D3p & 180 \\
D3m & 160 \\
H1T + H1B & 15 \\
H2T + H2B & 10 \\
H3T + H3B & 10 \\
H4T + H4B & 10 \\
Proportional tubes & 300 \\
\hline
\end{tabular}

Table 3.4: Cuts on number of hits of detecors.

tracking.

Other than the data trimming procedures discussed previously, kTracker also performs the trigger analysis, with which all possible trigger roads are enumerated by matching the corresponding X-plane-hodoscope hits. The hodoscope hits that are not associated with any road candidates are removed. In addition, once an event is found to have five or more possible $\mu^{+}$or $\mu^{-}$roads, this event is discarded based on the time-consumption criteria mentioned above. The proportional tube hits are also analyzed to form "tracklets", which are defined as local tracks inside the detector and are used as seeds for reconstructing global tracks. These tracklets are then stored for future muon identification.

\subsubsection{Track Reconstruction}

The next phase of tracking is track reconstruction and begins with identifying tracklets inside individual chambers. Starting from the station 2 and 3 chambers, the tracker looks for tracklets as hit triplets of the three different views of a chamber. The steps of reconstructing a triplet are illustrated in Fig. 3.1. In the first step, the hit pairs, which are adjacent hits in the primed and unprimed planes, are picked out in the X-view. These hit pairs, together with the unpaired hits are used to define windows utilized in the next step. For a specific window, the U-view hits of wires overlapping spatially with this window are then associated with the $\mathrm{X}$-view hits to form $\mathrm{X}-\mathrm{U}$ 

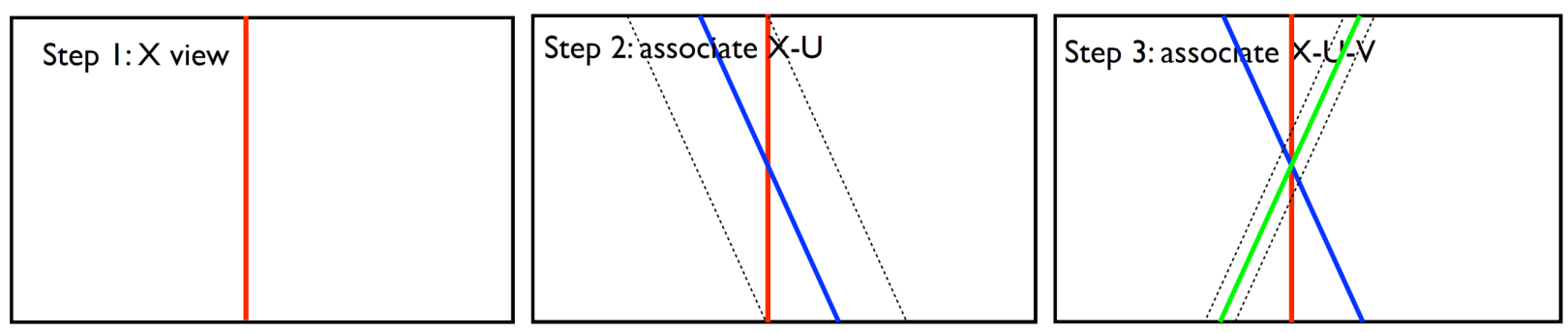

Figure 3.1: Steps for reconstructing of a local hit triplet in a chamber [109].

doublets, as shown in the center plot of Fig. 3.1. Analogously, much tighter windows specified by $\mathrm{X}-\mathrm{U}$ doublets are employed to determine the associated V-view hits and the triplets are formed. Subsequently, these triplets of hits are fitted to a line, assuming the spatial resolution of wires is determined by the wire spacing. A triplet is rejected if one of the condition listed below is met:

- The total fitting $\chi^{2}$ (d.o.f equals to 4 ) of the corresponding tracklet is larger than 15.

- The tracklet has less than 4 associated hits, or the hits from a specific view are missing.

- The tracklet does not point roughly towards the target.

- The nearest X-hodoscope paddle, which the tracklet is projected to, is not fired.

Once the station-2 and station-3 triplets are obtained, the connection between triplets from these two stations is attempted by making all possible tracklet combinations. Some cuts are applied to discard the combinations of bad quality, and the combinations that survive are then sent to a $\chi^{2}$-based fitter to form partial tracks between stations 2 and 3 . If there are two partial tracks that have more than $1 / 3$ of the hits in common, only the one with smaller $\chi^{2}$ is kept. Also, a tracklet combination is thrown away if its associated track is not pointing toward the target, or not pointing to fired paddles on station-2, 3, and 4 hodoscopes. Furthermore, a muon-identification cut is imposed to require the extrapolation of a partial track to station- 4 has matched proportionaltube hits, and a corresponding small deflection is observed due to passing through the iron absorber 

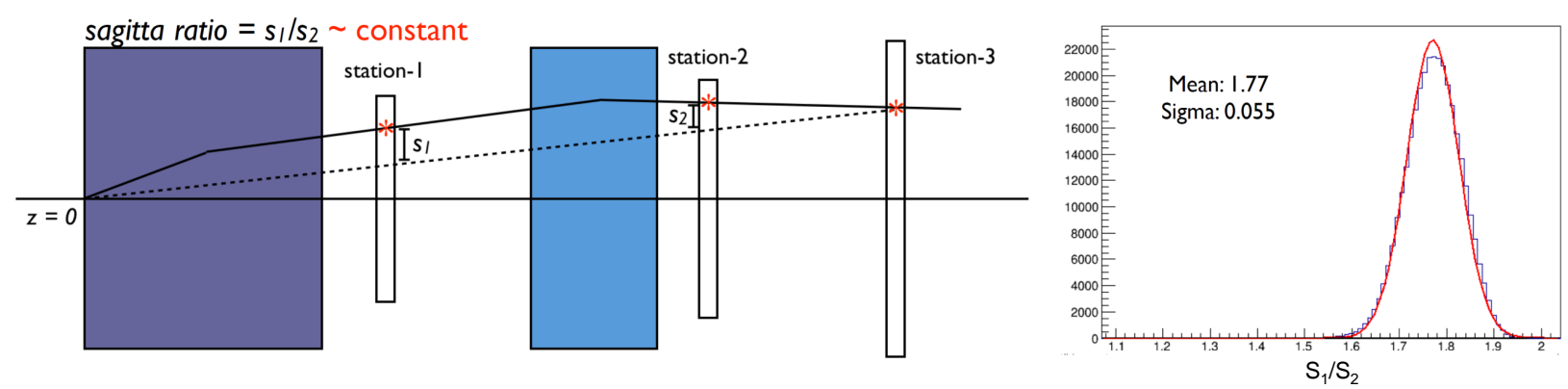

Figure 3.2: Track projection to station 1 by sagitta ratio [109].

wall. The retaining partial-track candidates are then projected upstream to station 1 by the "sagitta ratio" method to provide a search window for building station-1 tracklets. As demonstrated in the left plot of Fig. 3.2, the sagitta is defined as the distance between the track and the line connecting $\mathrm{X}=\mathrm{Y}=\mathrm{Z}=0$ and the station-3 triplet. For a high-momentum track, the effect of the magnetic fields can be approximated as providing transverse-momentum kicks around the center of magnets, and the resulting deflection of the track in the X-Z plane leads to a roughly constant ratio of $s_{1} / s_{2}$. The right plot of Fig. 3.2 shows the Monte Carlo simulation result of $s_{1} / s_{2}$, which can be described by a Gaussian distribution of small $\sigma$. The sagitta ratio is then applied for determining a window of $\pm 5 \mathrm{~cm}$ wide for station- 1 hits. The station- 1 hits that falls within the window are utilized to build station- 1 triplets and then connected to the associated station-2-to-3 partial track to form a global-track candidate.

To reconstruct global tracks, the first step is to clean out the bad hits iteratively; after fitting a track candidate, the hit having the largest residual is discarded if the residual is greater than three times the chamber resolution and the remaining hits are re-fit. This process is repeated until all the hits of a global track candidate have residuals smaller than three times of the resolution (7 $\mathrm{mm}$ ). The track candidates are then required to pass quality cuts that ensures tracks: (1) point to fired X-hodoscope paddles in all stations, (2) have momentum greater than $5 \mathrm{GeV}$ and less than 100 $\mathrm{GeV},(3)$ have at least four hits in each station and one hit in each view, and (4) not be deflected 
by the absorber wall more than expected according to its momentum. The tracks passing the cuts are then fine fitted by utilizing the Kalman filter algorithm.

The Kalman filter is the linear estimator that evaluates the dynamical evolution of states and finds the optimal estimation based on the information provided by imperfect and noisy measurements. Starting with an initial state vector with the uncertainty defined, the Kalman-filter algorithm propagates the state based on the physical model specified, and projects an estimation of the state variables. The estimation is subsequently combined with the outcome of the measurement using a weighted average, which involves their associated uncertainties and more weight is given to the one with higher certainty. The combined, updated state variables acquired are then propagated to generate a new estimation that will be corrected by the arrival of the result of next measurement. After a series of measurement inputs to this recursive process, an appropriate state-vector prediction and the corresponding error covariance matrix is provided by the Kalman filter.

The idea of using a Kalman filter in track fitting is demonstrated in Fig. 3.3. In the implementation of the Kalman-filter, a state vector consists of the spatial coordinates of a muon and its three momentum. The evolution of the state therefore corresponds to the change of parameters describing the muon as it penetrates through the spectrometer. The hit position at each detector plane, together with the corresponding resolution as uncertainty, is then treated as the measured input for the Kalman filter. Rather than using an analytical model, the dynamic propagation between states is fulfilled by employing the GEANT4 software package [110, with which the passage of muons through various materials in the spectrometer can be accurately simulated. It is also worth noting that the way a state is propagated in the kTracker is from the downstream end of the spectrometer towards the upstream. And the main motivation for this backwards-in-time approach is that the downstream part of the spectrometer is essentially free from the magnetic fringe field and less affected by the background particles generated upstream. For these reasons, the initial state variables and their propagation can be better defined, which then leads to a better convergence of the Kalman-filter estimation. 


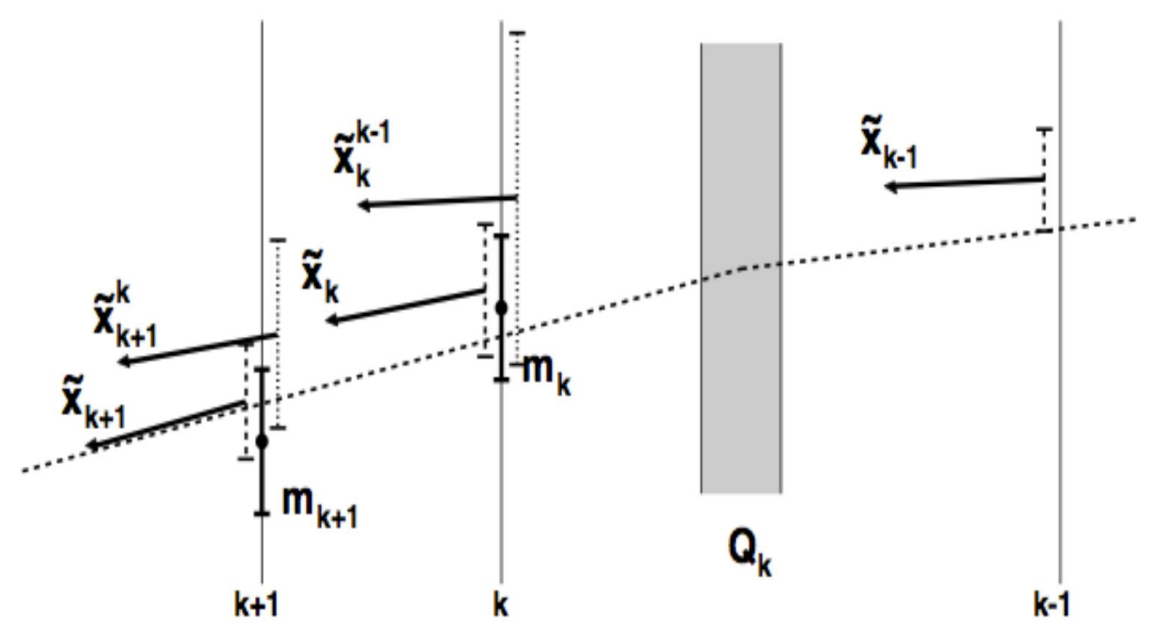

Figure 3.3: Demonstration of Kalman filter implementation for track fitting [109]. The estimations are denoted as $x$ and the measurements as $m$. The lower index shows the correlated detector plane and the upper index indicates the estimation at the current plane projected from the previous plane. The measurement-corrected estimation is given as the $x$ without an upper index.

The workflow of the fine track-fitting is illustrated in Fig. 3.4. After the initial estimation starting at the downstream end of a track, the process goes through the two-stage recursion of Kalman filter: in the prediction stage, the state from previous plane is propagate to the current plane. The measurement with the current plane is thus used to correct the predicted state in the correction stage. With all the hits associated with a track being looped through, the kTracker reverses the backwards-in-time process and propagates the muon in a forwards-in-time manner, from upstream to downstream, so that the state variables are continuous and smoothed. Finally, for the fitted global tracks that have converging $\chi^{2}$ values, they are passed to the vertex finding stage.

\subsubsection{Vertex finding}

Before the vertex-finding takes place, the muon tracks need to swim through the FMAG to be projected back to the target region. In the treatment of single-muon swimming, the whole FMAG is divided into many slices in the $\mathrm{Z}$ direction. While stepping through each slice as demonstrated 


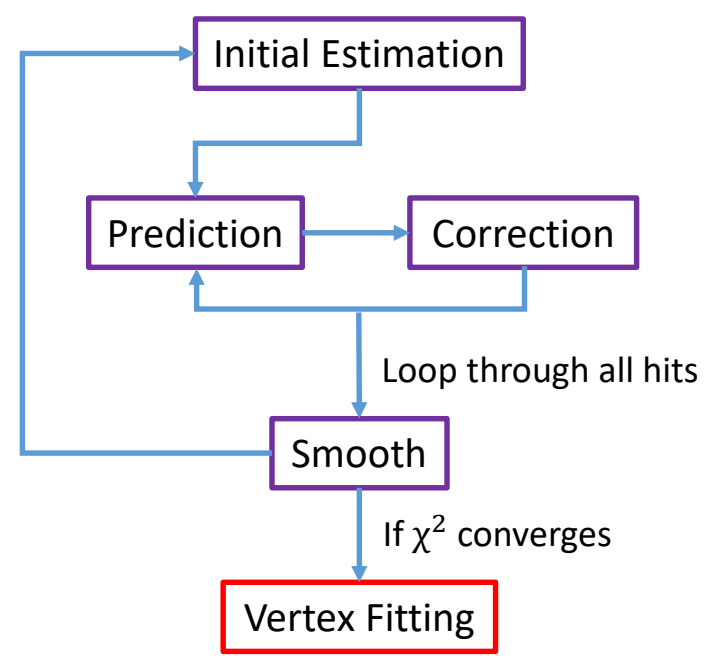

Figure 3.4: The workflow of Kalman-filter based track fitting

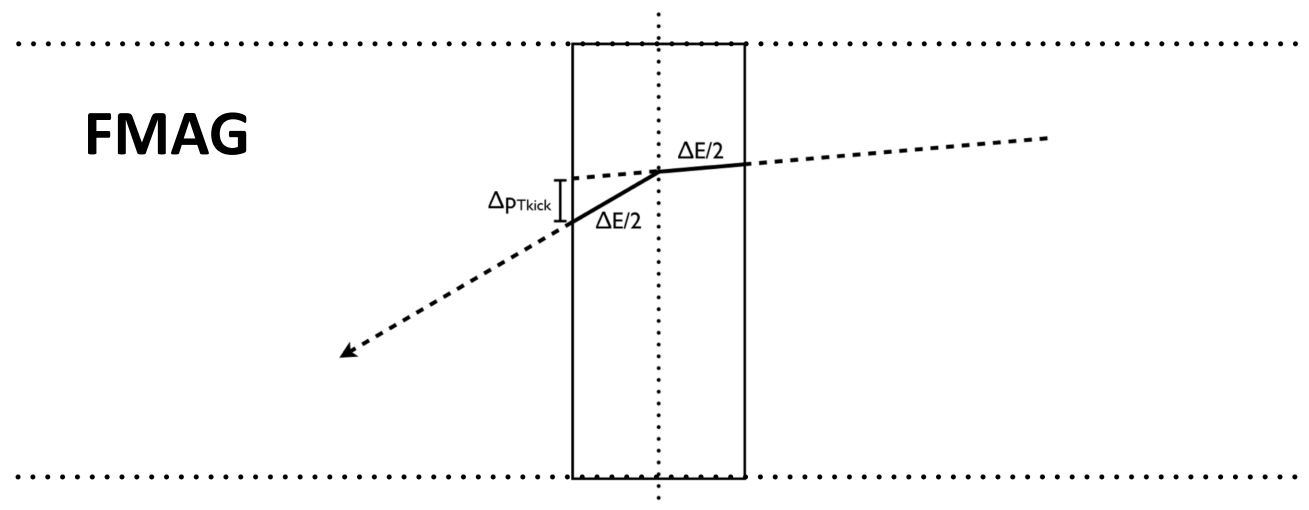

Figure 3.5: Treatment of a muon swimming through a single slice of FMAG.

in Fig. 3.5, the energy loss of the muon is compensated through the whole slice and a transversemomentum kick is applied at the center of the slice. After all the slices are swum through, the muon tracks are extrapolated to the target region. During this whole process, the position on a track that has the closest approach to the beam line is considered to be the "vertex" of the track.

The dimuon-vertex-finding is based on the extended Kalman filter method developed by Gorbunov and Kisel [11]. The dimuon vertex position is the state vector to be estimated by 
the Kalman filter. The muon tracks are paired up and every track is treated as an independent measurement of the vertex position. Once the Kalman-filter processing is performed, the updated vertex position is examined to see if the result is converging and falls in a reasonable Z-position range. If not, the Kalman filter procedure is repeated using the new vertex position as input until the number of iteration reaches the pre-defined limit. In the kTracker implementation of this vertex-finding algorithm, the initial vertex position is set on $\mathrm{X}=\mathrm{Y}=0$ with two different $\mathrm{Z}$ positions attempted: the average Z-positon value of the vertices of the two muon tracks, or the $\mathrm{Z}$ position where the two muon tracks are closest. Only the one with better vertex-fitting $\chi^{2}$ result is kept.

\subsection{Event Selection}

The track-reconstructed events are then required to pass through some data-quality cuts. The goal of these cuts is to filter out possible background events at our best, in the same time retain as many valid dimuons coming from the targets as possible to have them further analyzed. In this section, the selection rules, or "cuts", applied in this analysis will be introduced in a sequence from top to bottom based on the scale of the data involved, and from the reconstructed tracks to the dimuons.

\subsubsection{Spill-level and Event-level Cuts}

The spill-level cuts are used to exclude data from proton spills that have scaler readings falling outside a normal range, or have questionable process variables registered. The details of the range of cuts used are given in Tab. 3.5 and the meaning of some variables in the table is explained as the following. The "target position", as listed in Tab. 2.1, indicates which target is taking the proton beam during the spill. "TSGo" is the total number of triggers, regardless of the trigger type, received by the trigger supervisor. The number of accepted and inhibited FPGA-1 triggers are "AcceptedFPGA1" and "AfterInhFPGA1", separately. "G2SEM" gives the proton intensity measured by the SEM detector in G2 sector of the beam line. The "Duty Factor" is the 53-MHz duty factor defined as $\langle I\rangle^{2} /\left\langle I^{2}\right\rangle$, which provides the proton-beam quality. "QIEsum" is the whole- 


\begin{tabular}{lll}
\hline Variable & Roadset $57 \& 59$ & Roadset $62 \& 67$ \\
\hline Target position & $1-7$ & $1-7$ \\
TSGo & $1000-8000$ & $100-6000$ \\
AcceptedFPGA1 & $1000-8000$ & $100-6000$ \\
AfterInhFPGA1 & $1000-30000$ & $100-10000$ \\
AcceptedFPGA1/AfterInhFPGA1 & $0.2-0.9$ & $0.2-1.05$ \\
G2SEM & $2 \mathrm{e} 12-1 \mathrm{e} 13$ & $2 \mathrm{e} 12-1 \mathrm{e} 13$ \\
Duty factor & $15-60$ & $10-60$ \\
QIEsum & $4 \mathrm{e} 10-1 \mathrm{e} 12$ & $4 \mathrm{e} 10-1 \mathrm{e} 12$ \\
Inhibit & $4 \mathrm{e} 9-1 \mathrm{e} 11$ & $4 \mathrm{e} 9-2 \mathrm{e} 11$ \\
Busy & $4 \mathrm{e} 9-1 \mathrm{e} 11$ & $4 \mathrm{e} 9-1 \mathrm{e} 11$ \\
\hline
\end{tabular}

Table 3.5: Summary of cuts on spills. A list of allowed ranges of different variables is given. The allowed ranges are changed for different Roadsets due to different run conditions.

spill intensity sum measured by BIM. "Inhibit" means the intensity sum of buckets vetoed by the QIE inhibit, and "Busy" gives the total intensity of buckets blocked by TS busy.

Other than the cuts listed in Tab. 3.5, it is also required that the target position registered in different MySQL tables should be identical, there needs to be at least one kTrack entry in the spill, and all quantities used in the cuts must be in the data base with no more than one entry for the spill. These are used to ensure that there is no mistake made in the downstream data managing. For the spills that pass the spill level cuts, the event-level cuts are further applied to ensure that the events are FPGA-1 triggered and have been successfully processed by the kTracker.

\subsubsection{Track-level Cuts}

The track-level cuts, as summarized in Tab. 3.6, are applied to select out the reconstructed tracks of good quality. As listed in the table, an authentic track should be associated with a valid trigger-road ID, with a sufficient number of chamber hits, and a low-enough reduced- $\chi^{2}$ value. The vertex of a track, as defined previously, is the point of closest approach to the beam line, and the Z-position of it is required to lie in the range around the target and the beam dump. There is one additional cut that demands the Z-momentum of a track at Station 1, pz1, be larger than 18 


\begin{tabular}{ll}
\hline Variable & Range \\
\hline Road ID & $\neq 0$ \\
Number of hits & $>14$ \\
Reduced $\chi^{2}$ & $<5$ \\
Z position of vertex $(\mathrm{cm})$ & $(-400,200)$ \\
pz1, where number of hits $<18(\mathrm{GeV})$ & $>18$ \\
\hline
\end{tabular}

Table 3.6: Summary of cuts on tracks.

$\mathrm{GeV}$ if its associated chamber hits is less than 18. This rather awkward cut is utilized for now to significantly reduce random backgrounds at high dimuon-mass region, while a different approach is being investigated by the collaboration.

\subsubsection{Dimuon-level Cuts and Target Dimuon Selection}

To pick out valid dimuons, the dimuon-level cuts shown in Tab. 3.7 are subsequently applied. The "track separation" is defined as the Z-position separation between the vertices of the $\mu^{+}$and $\mu^{-}$tracks, and the $\chi^{2}$ of dimuon vertex reconstruction gives a measure of how well the two tracks converge to a single vertex. Applying cuts on these two quantities ensures that the two muon tracks are correctly associated with a single origin, a promising dimuon. The requirements on the dimuon-vertex positons guarantee that the dimuons are produced near the passage of beam and close to the target or dump. The dimuon-momentum-at-vertex cuts are applied based on Monte Carlo studies which show: (1) a true dimuon with momentum too low would simply be swept away by the magnets and fall out the acceptance of SeaQuest spectrometer, and (2) the large multiple scattering if low momentum muons leads to large uncertainties in the vertex position. Since the energy of the proton beam is $120 \mathrm{GeV}$, an additional upper bound of $120 \mathrm{GeV}$ on the Z-momentum is placed. Similarly, the requirements on the signs of the $\mu^{+}$and $\mu^{-} \mathrm{X}$-momentum is due to the fact that tracks carrying the wrong sign should be bent out by the magnets and are not focused into the detector acceptance. Lastly in the table, the cuts on $x_{1}, x_{2}$, and $x_{F}$ make sure that these quantities are physically legitimate. 


\begin{tabular}{ll}
\hline Variable & Range \\
\hline Track separation $(\mathrm{cm})$ & $(-250,250)$ \\
$\chi^{2}$ of dimuon vertex reconstruction & $<15$ \\
X position of dimuon vertex $(\mathrm{cm})$ & $(-2,2)$ \\
Y position of dimuon vertex $(\mathrm{cm})$ & $(-2,2)$ \\
Z position of dimuon vertex $(\mathrm{cm})$ & $(-300,200)$ \\
X momentum at dimuon vertex $(\mathrm{GeV})$ & $(-3,3)$ \\
Y momentum at dimuon vertex $(\mathrm{GeV})$ & $(-3,3)$ \\
$\mathrm{Z}$ momentum at dimuon vertex $(\mathrm{GeV})$ & $(30,120)$ \\
$\mathrm{X}$ momentum of $\mu^{+}(\mathrm{GeV})$ & $>0$ \\
$\mathrm{X}$ momentum of $\mu^{-}(\mathrm{GeV})$ & $<0$ \\
$x_{1}$ & $(0,1)$ \\
$x_{2}$ & $(0,1)$ \\
$x_{F}$ & $(-1,1)$ \\
\hline
\end{tabular}

Table 3.7: Summary of cuts on dimuons.

At this stage, with the dimuon events that passed through all the cuts given previously, two more cuts are required to distinguish the dimuons originating from the target to the ones from the dump or other places. The first one is based on the $\chi^{2}$ values of a track by forcing the vertex of this track to be at a specific location in the target region or the dump region. These positons are defined as $X=0, Y=0$, and $Z=-129.5 \mathrm{~cm}$ for the evaluation of $\chi_{\text {Target }}^{2}$, or $Z=42 \mathrm{~cm}$ for $\chi_{\text {Dump }}^{2}$. These two $\chi^{2}$ values are calculated for each track and if both dimuon tracks have

$$
\chi_{\text {Dump }}^{2}-\chi_{\text {Target }}^{2}>10
$$

then this dimuon is regarded as the dimuon produced from the target. Otherwise, if the tracks have

$$
\chi_{\text {Target }}^{2}-\chi_{\text {Dump }}^{2}>10
$$

the corresponding dimuon is considered to be coming from the dump. The Monte Carlo study of this cut is illustrated by the plot on the left of Fig. 3.6. where the red data points are generated at the target region and the black ones are at the dump region. The effect of applying these cuts on the vertex Z-postion distribution of the dimuons is show in the right plot of Fig. 3.6, in which a decent target-dump separation is achieved. An additional cut that requires the Z-position to sit 

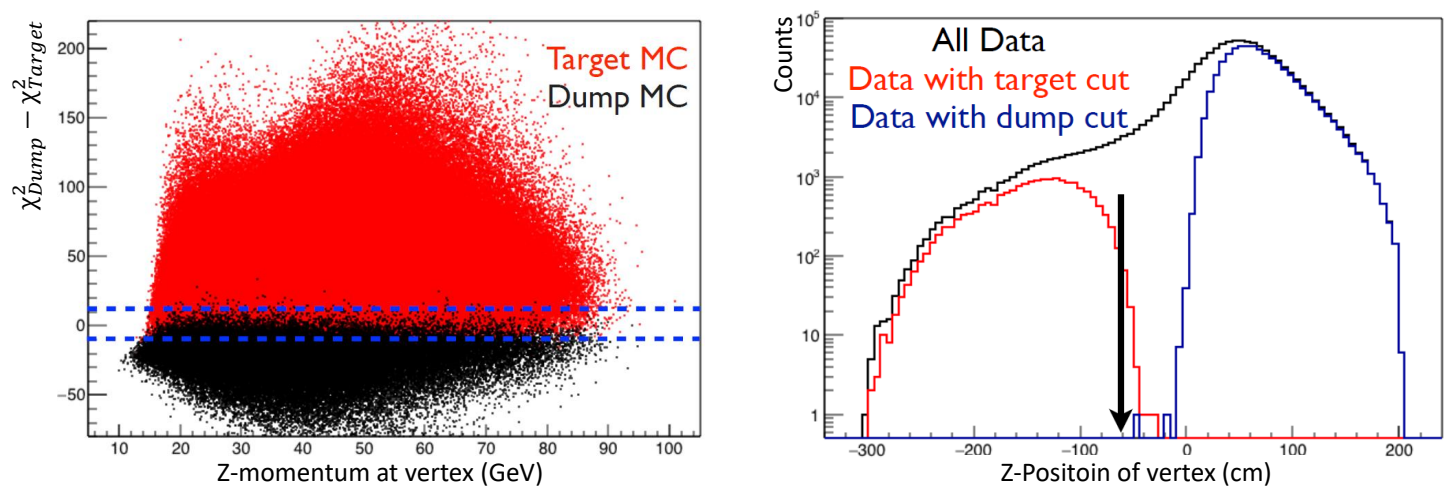

Figure 3.6: Target-dump separation of dimuons [112]. The left plot shows the $\chi_{\text {Dump }}^{2}-\chi_{\text {Target }}^{2}$ versus the dimuon Z-momentum, and the cuts for separating target or dump dimuons are given as blue dash lines. The plot on right shows the Z-position of the dimuons with the $\chi^{2}$-separation cuts applied, in which the black arrow points at $Z=30 \mathrm{~cm}$.

\begin{tabular}{ll}
\hline Variable & Range \\
\hline$\chi_{\text {Dump }}^{2}-\chi_{\text {Target }}^{2}$ & $>10$ \\
$Z$ position of dimuon vertex $(\mathrm{cm})$ & $(-300,-60)$ \\
\hline
\end{tabular}

Table 3.8: Summary of cuts on target dimuons.

between -300 and $-60 \mathrm{~cm}$ is imposed to assure the validity of a target-originating dimuon. The cuts utilized for target-dimuon selection is summarized in Tab. 3.8 .

\subsection{Monte Carlo Simulation}

The Monte Carlo Simulation program (MC) is used to simulate the outcome of the experiment. Two different Monte Carlo Simulation programs were developed for SeaQuest. The fast Monte Carlo (FastMC) adopted the one employed by the E866 experiment and has been used to provide fast but rather rough analysis results. The GEANT Monte Carlo (GMC), implemented with the GEANT4 toolkit, is utilized to provide a detailed simulation on effects such as the penetration of muons and other particles, the fringe field of the magnets, and the response of the spectrometer.

The dimuon events are generated with a flat, equal-probability distribution over the mass, $M$, 

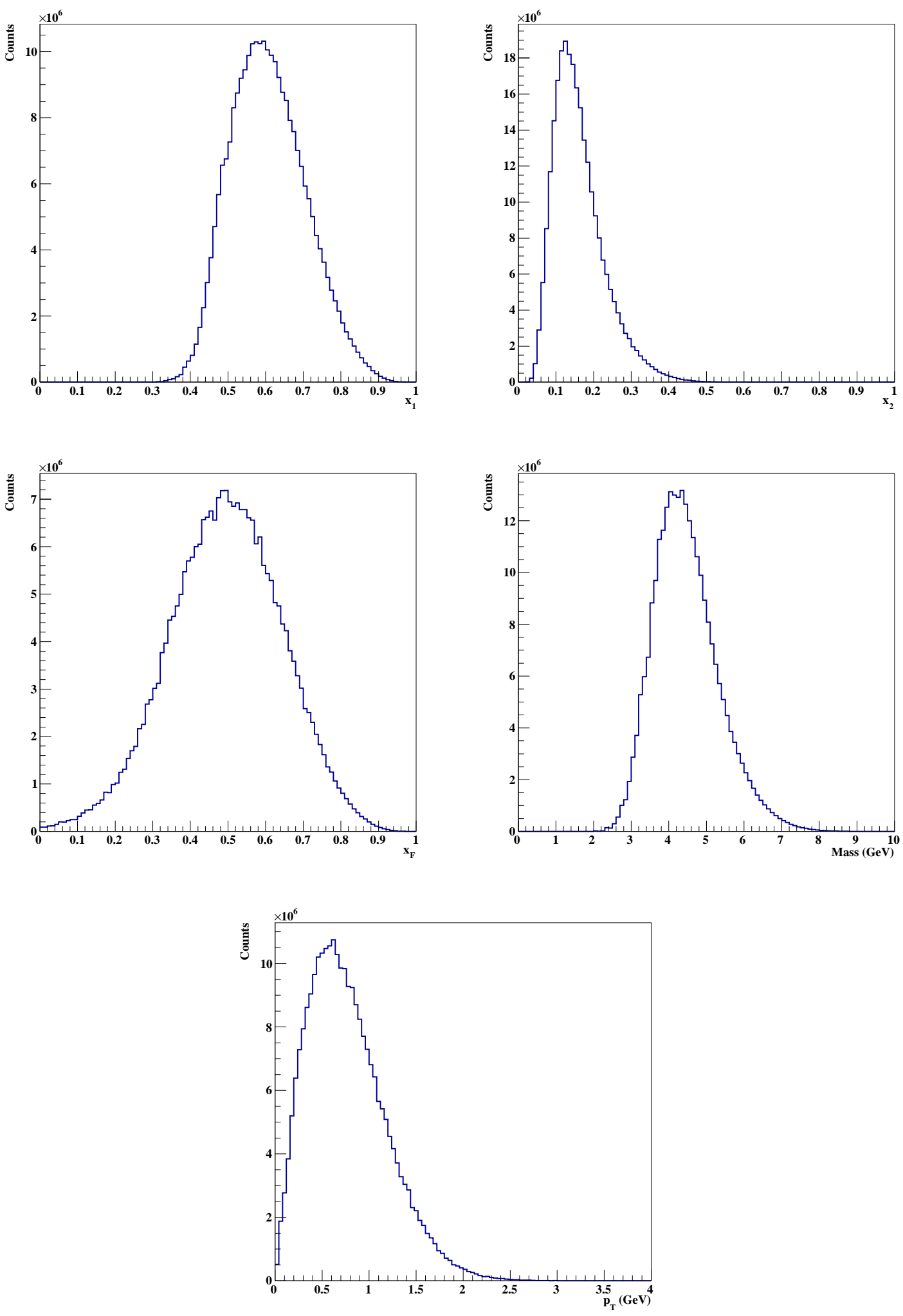

Figure 3.7: Kinematic distributions of the Monte Carlo events. 
and Feynman-x, $x_{F}$, in the range of $1<M<10 \mathrm{GeV}$ and $-1<x_{F}<1$. The transverse momentum $p_{T}$ and the Collins-Soper $\theta$ and $\phi$ are produced according to their own parameterized distributions, such as the Kaplan formula [113] used for $p_{T}$. The Bjorken-x's are accordingly calculated and the kinematic cuts are subsequently imposed so that $0<x_{1}<1,0<x_{2}<1$, and $p_{T}^{2}$ are restricted. The Z-position of dimuon generation along the beam is chosen according to a distribution based on beam attenuation. Once an event is generated, a corresponding weight is evaluated based on the leading-order cross section, which incorporates only quark flavors of up, down, strange, and charm, and is not affected by the $p_{T}$ and the Collins-Soper angles. The weight obtained is subsequently assigned to this event. The PDF sets from the CTEQ-TEA collaboration have been adopted [70] in the cross-section calculation. While GMC is capable of producing Drell-Yan, $J / \psi$, and $\psi^{\prime}$ dimuons, the FastMC has been adopted for the generation of Drell-Yan dimuons. The distributions of some kinematic variables from Monte Carlo are shown in Fig. 3.7.

\subsection{Per-nucleon Cross Section Ratios}

As mentioned before, a signature of energy loss is the modification in the differential cross section for a proton hitting on the heavy nuclear target. In this analysis, we measure the Drell-Yan differential cross section $d \sigma / d x_{F}$ of the heavy nuclear targets, iron and tungsten, and normalize them by the one with the carbon target. The per-nucleon $d \sigma / d x_{F}$ ratio, which is expected to show a trend of suppression at high $x_{F}$ due to the energy loss, is defined as

$$
R_{p A}=\left(\frac{1}{A_{A}} \frac{d \sigma(A)}{d x_{F}}\right) /\left(\frac{1}{A_{C}} \frac{d \sigma(C)}{d x_{F}}\right)
$$

where the mass numbers represented by the $A$ 's. In the equation above and others given in the rest of this thesis, the subscript " $A$ " of various quantities is used for denoting the heavy nuclear targets and " $C$ " is for carbon, specifically. This subscript is sometimes omitted in discussions of general cases. The reasons for not using the deuterium target data, which is expected to have no observable energy loss effect involved, in the cross-section ratio evaluation are the following: 


\begin{tabular}{cc}
\hline Bin ID & $x_{F}$ Range \\
\hline 1 & $(0.00,0.30]$ \\
2 & $(0.30,0.41]$ \\
3 & $(0.41,0.50]$ \\
4 & $(0.50,0.60]$ \\
5 & $(0.60,0.67]$ \\
6 & $(0.67,1.00]$ \\
\hline
\end{tabular}

Table 3.9: $x_{F}$ binning definition.

- The deuterium target in Run-III has a non-negligible contamination issue. During Run-III , the deuterium target was emptied and refilled a few times. The purity of the deuterium bottles used was unstable and showed possible contamination either from air or from the bottles and flask that were not properly handled. The estimated systematic uncertainty brought by the contamination is on the order of $5 \%$.

- In the beginning of Run-III while the deuterium was still under preparation, SeaQuest decided to test the stability and consistency between the liquid targets so the data taking was performed with the deuterium flask filled with liquid hydrogen. A total of about 12,000 spills of data were collected in this period and the data taken with solid targets are valid for physics analysis.

- The carbon target, with mass number $A=12$, is still light enough that the energy loss effect can be treated as small compared to the ones of heavy nuclear targets [100].

Based on these facts, to obtain the most accurate measurement with the amount of data at hand, the carbon target is chosen as the denominator instead of the liquid deuterium.

To obtain $d \sigma / d x_{F}$, the cross-section ratio is evaluated in six $x_{F}$ bins. The range of the $x_{F}$ bins are chosen so that each bin would have roughly equal number of Drell-Yan events based on the Monte Carlo simulation. The $x_{F}$ binning definition is given in Tab. 3.9. 


\begin{tabular}{c|ccc}
\hline Target Material & Mass Number $A$ & Density $\rho\left(\mathrm{g} / \mathrm{cm}^{3}\right)$ & Nuclear Interaction Length $\left(\mathrm{g} / \mathrm{cm}^{2}\right)$ \\
\hline $\mathrm{LH}_{2}$ & 1.00794 & $0.071 \pm 0.001$ & 52.0 \\
$\mathrm{LD}_{2}$ & 2.01410 & $0.163 \pm 0.001$ & 71.8 \\
$\mathrm{Fe}$ & 55.845 & $7.87 \pm 0.01$ & 132.1 \\
$\mathrm{C}$ & 12.0107 & $1.80 \pm 0.01$ & 85.8 \\
$\mathrm{~W}$ & 183.84 & $19.30 \pm 0.01$ & 191.9 \\
\hline
\end{tabular}

Table 3.10: Target material properties.

Experimentally, the per-nucleon cross section ratio of the Drell-Yan process can be defined and measured as

$$
R_{p A}=N_{A / C} \cdot \frac{Y_{A}^{D Y}}{Y_{C}^{D Y}} \cdot F_{A / C}^{R D} \cdot F_{A / C}^{A c p} .
$$

In this expression, $N_{A / C}$ indicates the normalization factor, $Y_{D Y}$ is the yield of Drell-Yan events, $F_{A / C}^{R D}$ is the rate-dependence correction factor, and $F_{A / C}^{A c p}$ the correction factor of acceptance. The way that each term is acquired in SeaQuest, especially $Y_{D Y}$ and $F_{A / C}^{R D}$, is explained in further detail in the rest of this chapter. Starting with $N_{A / C}$, this normalization factor that can be expressed as

$$
N_{A / C}=\frac{A_{C}}{A_{A}}\left(\frac{n_{C} L_{C}}{n_{A} L_{A}}\right) \cdot\left(\frac{N_{C}^{P-e f f}}{N_{A}^{P-e f f}}\right) .
$$

In the expression above, the $A$ is the mass number. The first term in the round brackets, $n L$, is the product of the volume density of the nucleons $n$ and the length of the target $L$. This quantity can be understood as the number of target nucleons per unit area seen by the incident protons. $n$ can be derived as $n=N_{0} \rho / A$, in which $N_{0}$ is the Avogadro constant, $\rho_{A}$ the density, and $A_{A}$ the mass number of the corresponding target. The values of these quantities can be found in Tab. 2.1 and 3.10 .

The second term in the round brackets of Eq. 3.3. $N^{P-e f f}$ is the effective number of protons interacting with the targets. Since the number of incident protons decreases as the protons propagate through a target due to other nuclear interactions, rather than using the total number of the incident protons, $N^{P-e f f}$, which accouts for the lost of protons, needs to be derived. The calculation of $N_{A}^{P-e f f}$ is not straightforward and will be discussed in more detail. 


\begin{tabular}{c|ccc}
\hline Target & Run-II & Roadset 62 & Roadset 67 \\
\hline Fe & 0.527 & 0.644 & 1.785 \\
C & 1.625 & 1.233 & 3.649 \\
W & 0.541 & 0.649 & 1.818 \\
\hline
\end{tabular}

Table 3.11: Number of live protons for each target in each data set, given in $\times 10^{16}$ protons. The uncertainties are estimated to be $1 \%$ of the numbers listed based on the performance of the QIE module.

\subsubsection{Effective Number of Protons Evaluation}

To acquire the effective number of protons $N^{P-e f f}$ used in Eq. 3.3 , we need to first evaluate the quantity called live proton, $N^{P-l i v e}$, which excludes the protons in the buckets for which the trigger is inhibited or the DAQ is in busy status. The G2SEM can reliably measure the proton number in a whole spill but cannot resolve the beam intensity bucket by bucket. The information provided by the beam intensity monitor (BIM) is therefore required. By using the coincidence of the BIM record and the trigger-supervisor inhibit/busy signal, the measurement of the number of protons lost due to the beam inhibit and DAQ busy is provided. Together with the absolute measurement from the G2SEM, the number of live protons $N^{P-l i v e}$ on each target can be calculated with the following formula

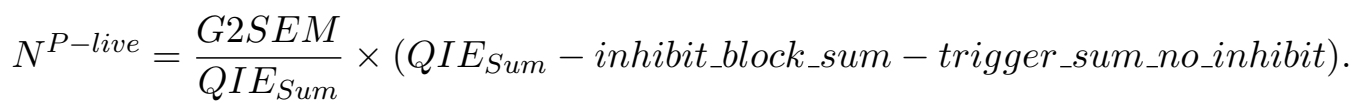

In this expression, G2SEM simply indicates the number of protons measured by G2SEM and the detailed description for the other quantities can be found in Section 2.1.1 where the outputs of BIM is explained. The $N^{P-l i v e}$ values of the solid targets in each data set are given in Tab. 3.11 .

Another factor that needs to be considered is the attenuation of the proton beam. As the incident protons travel through the target, the number of protons gradually decreases due to inelastic nuclear interactions and the valid proton intensity is therefore not the same at different Z-positions along the target. The strength of this effect is target dependent and can be characterized by the nuclear interaction length given in Tab. 3.10. By dividing the nuclear interaction length with the 
density $\rho$ of the target, we can obtain a corresponding length $\lambda$. For a proton beam that travels a distance $l$ in this nuclear material, the intensity decreases by a factor of $e^{-l / \lambda}$. The average attenuation brought by the whole target of length $L$ to the live protons is evaluated and we acquire the effective number of protons $N^{P-e f f}$ as:

$$
N^{P-e f f}=N^{P-\text { live }} \cdot \frac{\int_{0}^{L} e^{-x / \lambda} d x}{L}=N^{P-\text { live }} \cdot \frac{\lambda\left(1-e^{-L / \lambda}\right)}{L},
$$

which is the value used for the normalization of the cross-section ratio evaluation.

\subsection{Background}

For the evaluation of the cross-section ratio, the most critical issue to be dealt with is the extraction of the Drell-Yan event yield in each $x_{F}$ bin. In Eq. 3.2, the Drell-Yan yield $Y^{D Y}$ can be obtained as

$$
Y^{D Y}=N^{r a w}-N^{b k g}
$$

where $N^{\text {raw }}$ is the raw yield measured and $N^{b k g}$ is the number of background counts. Since our focus is on high-mass Drell-Yan events, there are three major types of "background" sources within the kinematic acceptance of SeaQuest experiment: (1) the $J / \psi(3097)$ resonance, $(2)$ the $\psi^{\prime}(3686)$ resonance, and (3) the events that originate from uncorrelated single muon tracks, denoted as "combinatorial background". In principle, the $J / \psi$ and $\psi^{\prime}$ events can be removed by requiring cut of dimuon mass > 4.2 GeV. However, a non-negligible combinatorial background can still exist in this high-mass region. To properly determine the background, different approaches are taken and the details about the methodology of background treatment will be explained in this section. In the rest of this chapter, the background mentioned simply refers to the combinatorial background only.

\subsubsection{Combinatorial Background}

As the beam protons come along the beamline, they can interact with the upstream instrumentation package, the targets, or the FMAG before being totally absorbed in the beam dump. A 
large number of mesons would therefore be generated. These mesons, mostly pions and kaons, can then decay and produce muons via processes like:

$$
\begin{gathered}
\pi^{+} \rightarrow \mu^{+}+\nu_{\mu} \\
\pi^{-} \rightarrow \mu^{-}+\bar{\nu}_{\mu} \\
K^{+} \rightarrow \mu^{+}+\nu_{\mu} \\
K^{-} \rightarrow \mu^{-}+\bar{\nu}_{\mu}
\end{gathered}
$$

The randomly-generated, uncorrelated muons from Drell-Yan, $J / \psi$, or these meson decays might accidentally form opposite sign dimuon pairs that satisfy the trigger and cut requirements, and then generate the background events. This type of background is referred to as the combinatorial background.

Since the cuts applied on the data would effectively remove dimuon events coming from the beam dump or the upstream materials and there is no additional flask material which needs to be considered in the liquid-target case, the background events of solid targets should be mostly dominated by the combinatorial background. Our current understanding of the combinatorial background suggests that a good fraction of this background comes from erroneously combining a track from the beam dump with a track from the target. Due to the fact that the single muons emerging from the interactions in the targets can also contribute to the combinatorial background, this background cannot be sufficiently represented by the data from the none target. Another option to generate the combinatorial background is to employ the Monte Carlo simulation. However, since the combinatorial background would depend on various factors like the detector efficiency and the condition of the beam, it is non-trivial to convincingly reproduce it by Monte Carlo.

In principle, the data taken by the NIM-3 random trigger can be used for studying the properties of the combinatorial background. The problem is that after imposing the trigger requirements, dimuon reconstruction, and cuts on the NIM-3 data collected so far, only a few hundred events survive and the statistics are too limited for meaningful analysis purposes. At this stage, the most practical way to produce the combinatorial background is to combine single tracks on our own. 
This the so-called "random mixing" method, which relies on the fundamental assumption that the muon tracks in a fake dimuon come from two different physics process and are completely independent of each other. This method uses the FPGA-1 triggered events in which only one single track is successfully reconstructed. To mimic the combinatorial background as realistically as possible, only the tracks collected from the same target in the same run are mixed together to form the random-mix data set.

To prepare the random-mix data of opposite sign muon pairs, the single tracks collected in a run are put in two separate track pools depending on their sign, sorted by their corresponding target, and indexed. For each target, a $\mu^{+}$track is picked out from the pool by its index, which is determined by a random number (ranging from 0 to 1 ) times the number of $\mu^{+}$tracks in the pool. The selected $\mu^{+}$track is then combined with a $\mu^{-}$track that is picked by the same method. The corresponding trigger roads of these mixed tracks are examined to ensure they form a top-bottom combination as required by the FPGA-1 trigger, and the separation of Z-position of the track are required to be smaller than $300 \mathrm{~cm}$. The track pairs that satisfy the requirements are saved and are not used again, and the track mixing process repeats until all available tracks are used. Although the statistics of the random-mix events that can pass all the cut requirements is still limited by the size of the data collected, it is sufficient for the combinatorial background study.

To justify this method of background treatment, which based on the combinatorial-backgrounddominant assumption, the validity of the background shape, together with the determination of the number of background need to be checked as will be discussed in the upcoming subsections.

\subsubsection{Background Shape}

For reasons that will be clear in Section 3.6.3, the determination of the number of background events heavily depends on the reliability of the background shape of the random-mix data, which is to say, the ability to produce the same kinematic distributions of the real combinatorial background. As a first check of the random-mixing method, we produce the so-called "like-sign random-mix data" with the same track-mixing procedures discussed in the previous subsection, but have the tracks of 
same sign of charge mixed together. The kinematic variable spectrum of the like-sign random-mix samples is then compared with that from the FPGA-3 like-sign trigger data. Since the FPGA-3 data is expected to be composed by random tracks, the kinematic agreement between the FPGA-3 data and the like-sign random-mix can assure the feasibility of the random-mixing method. The comparison of different variables, mass, $p_{T}, x_{1}, x_{2}$, and $x_{F}$ are shown in Fig 3.8 . Due to the prescale factor of FPGA-3 trigger and the cuts applied, the statistics of the FPGA-3 events is quite low. Nevertheless, the general consistency between the FPGA-3 and like-sign random-mix data can be seen.

Next, the background shapes of kinematic variables from the FPGA-1 trigger data are examined. However, there is no way to extract out the whole background shape of the real data sets before a full understanding of the background is gained. Therefore, we can only start within a limited kinematic region, which is the low mass part of the data, where most of the events in this region are combinatorial backgrounds.

Fig. 3.9 shows an example of the mass distribution of dimuons that pass the cuts required. As indicated in Fig. 2.8, SeaQuest is focused on the high mass dimuons and has a rapidly falling acceptance in the mass $<4 \mathrm{GeV}$ region. The number of $J / \psi$ events is therefore more suppressed than $\psi^{\prime}$ and the ratio of $\psi^{\prime}$ yield to that of $J / \psi$ is noticeably larger than one would expect from the production ratio. In the mass region smaller than $J / \psi$ the experiment basically has no acceptance for Drell-Yan events. As is suggested in Fig. 3.9. the events with mass $<2.5 \mathrm{GeV}$ are dominated by combinatorial background. To verify if the random-mix data describes the background well, the kinematic-variable comparison is performed between the real data and the random-mix data for all three data sets, just as is done with the like-sign mix and the FPGA-3 data. Fig. 3.10, 3.11, and 3.12 show the results of the solid targets. Although the statistics is limited by the mass $<2.5$ $\mathrm{GeV}$ requirement, it can be seen from the comparison plots that the kinematic dependence of the background is correctly reproduced by the random-mix data.

The authenticity of the mass spectrum of the random-mix data is especially important for the background estimation. In this analysis, the mass $>4.2 \mathrm{GeV}$ cut is used to remove events 

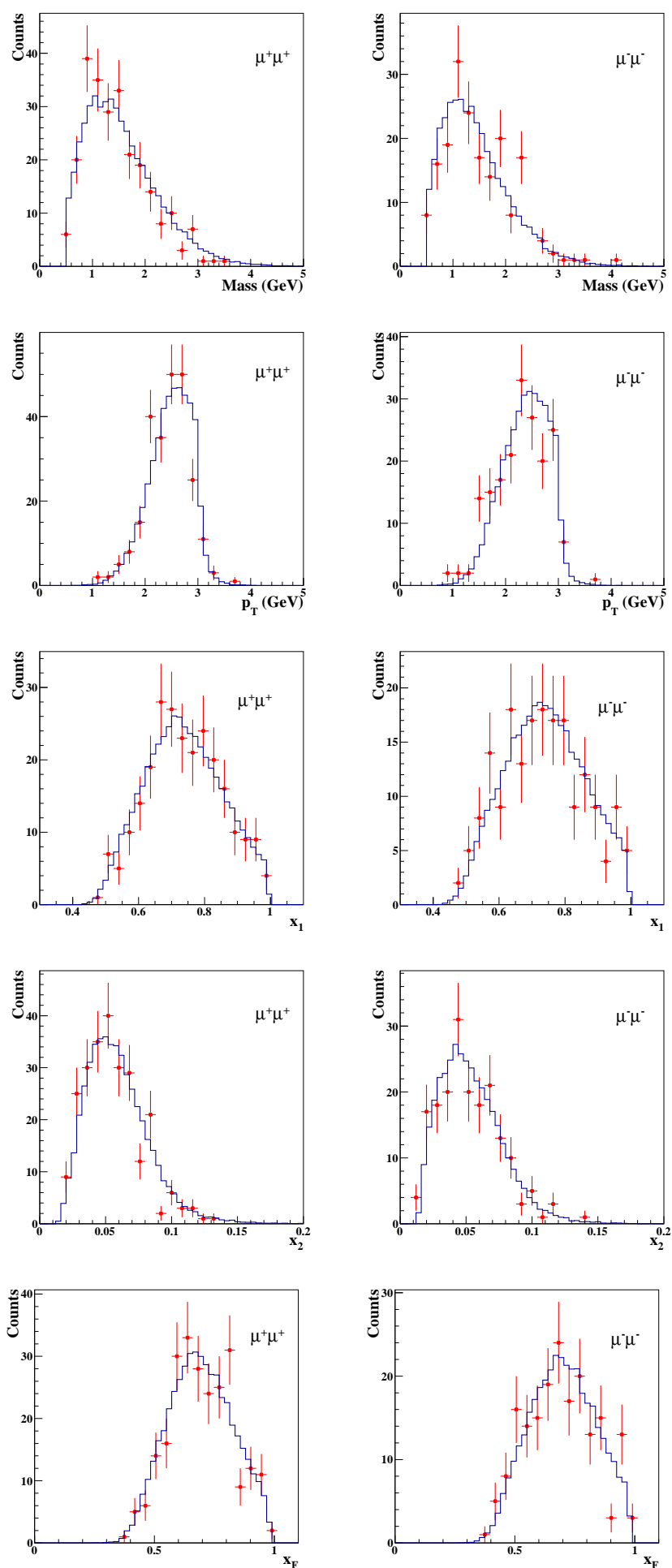

Figure 3.8: Comparison of kinematic variable distributions between like-sign random-mix and FPGA-3 data. The variables are mass, $p_{T}, x_{1}, x_{2}$, and $x_{F}$, from top to bottom. The plots in the left (right) column show the comparison of $\mu^{+} \mu^{+}\left(\mu^{-} \mu^{-}\right)$tracks. 

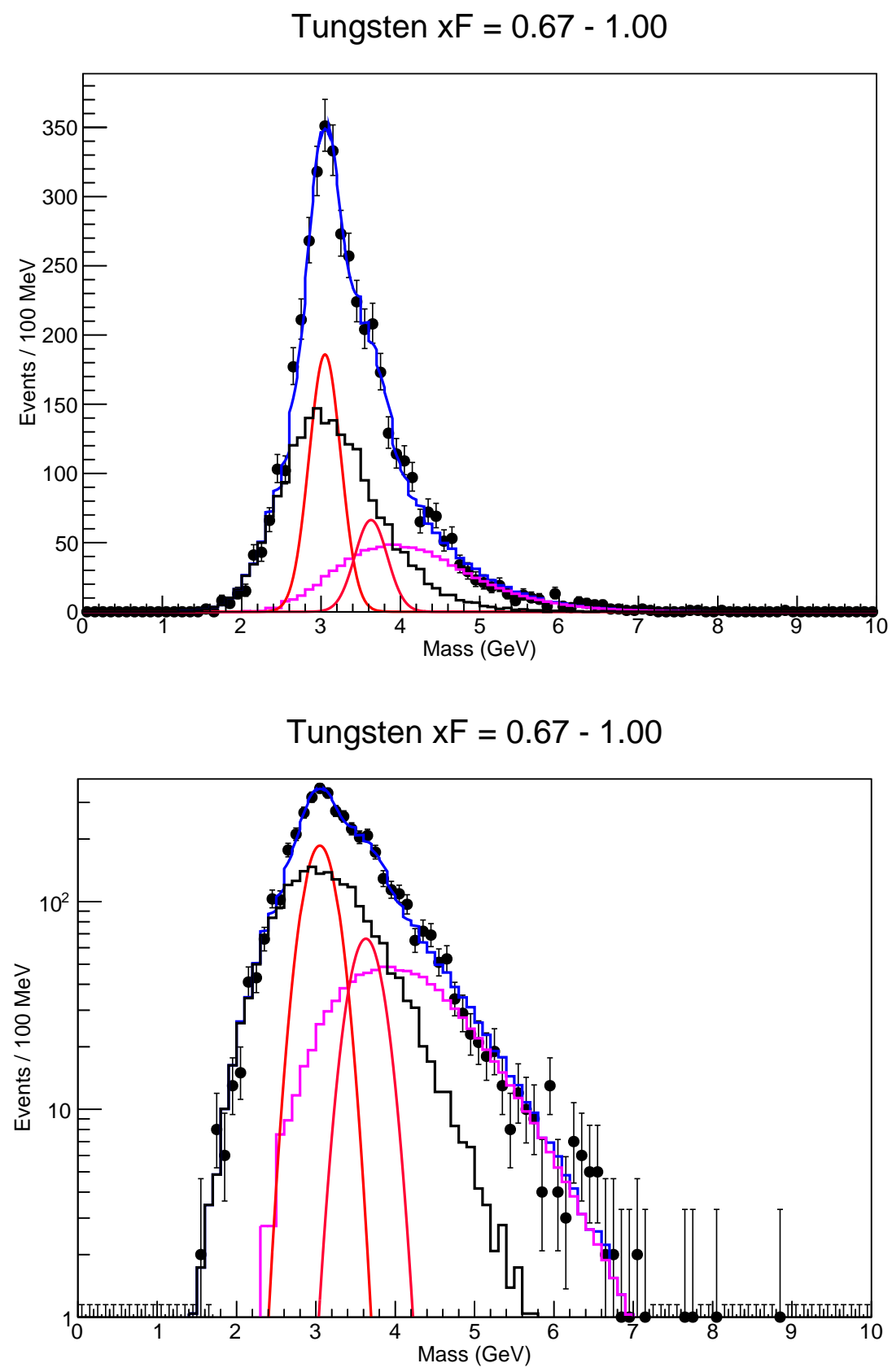

Figure 3.9: The mass spectrum of tungsten target data from Roadset 67 in the $x_{F}$ range of 0.67 to 1.00 and the fitting extracted contribution from different sources. The real data is shown as the black solid dots. The magenta line is the Drell-Yan Monte Carlo events. The red lines are $J / \psi$ and $\psi^{\prime}$. The black line is the random-mix data, which is used to represent the combinatorial background. The sum of all the sources is represented by the blue line. The bottom plot is the same as the top one, but the $y$-axis is plotted on the logarithmic scale. 

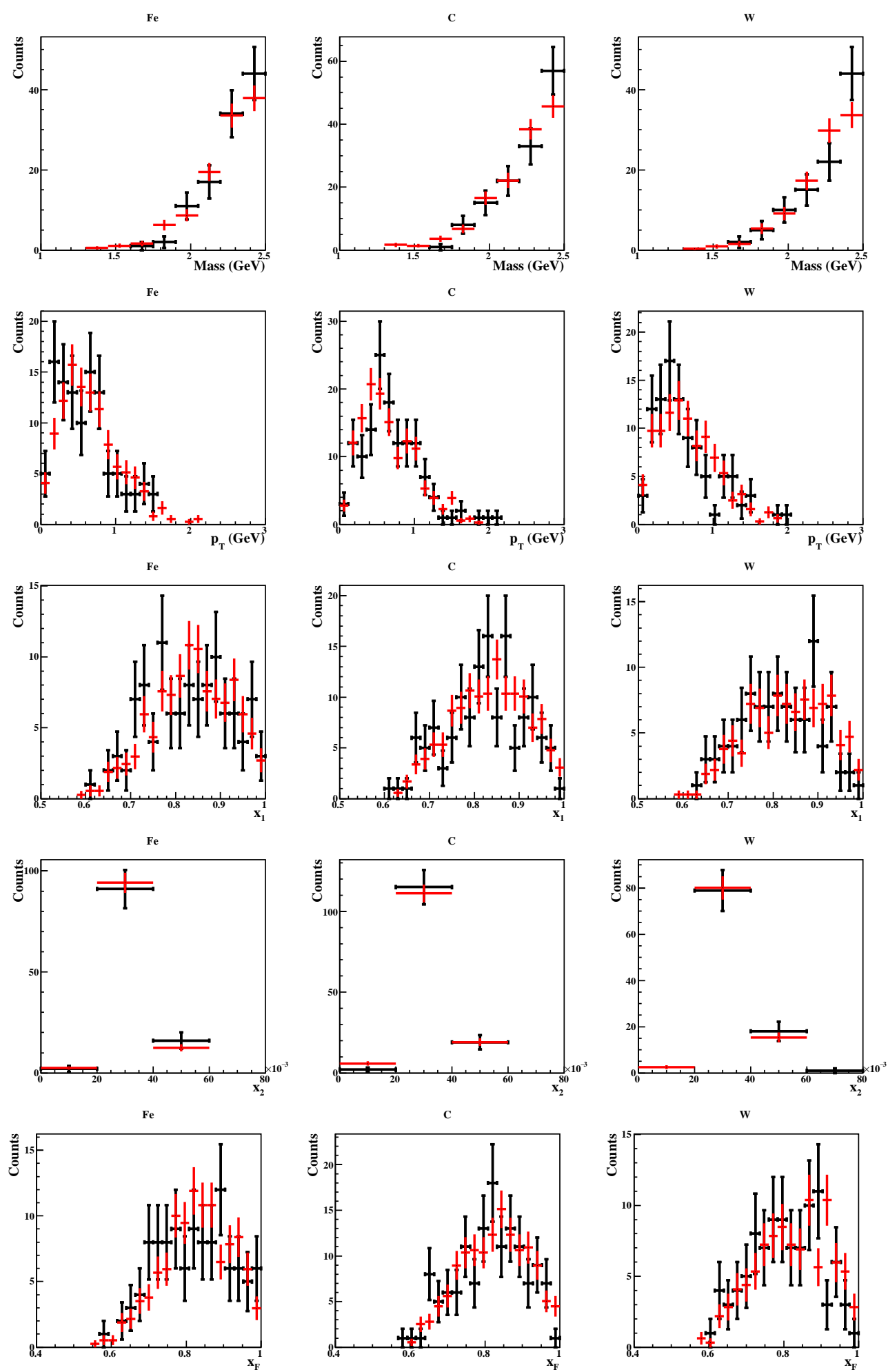

Figure 3.10: Comparison of distributions of kinematic variables of mass $<2.5 \mathrm{GeV}$ of Run-II data. The variables are mass, $p_{T}, x_{1}, x_{2}$, and $x_{F}$, from top to bottom. The black points are the real data, and the red points are the random-mix data scaled to the same normalization. 

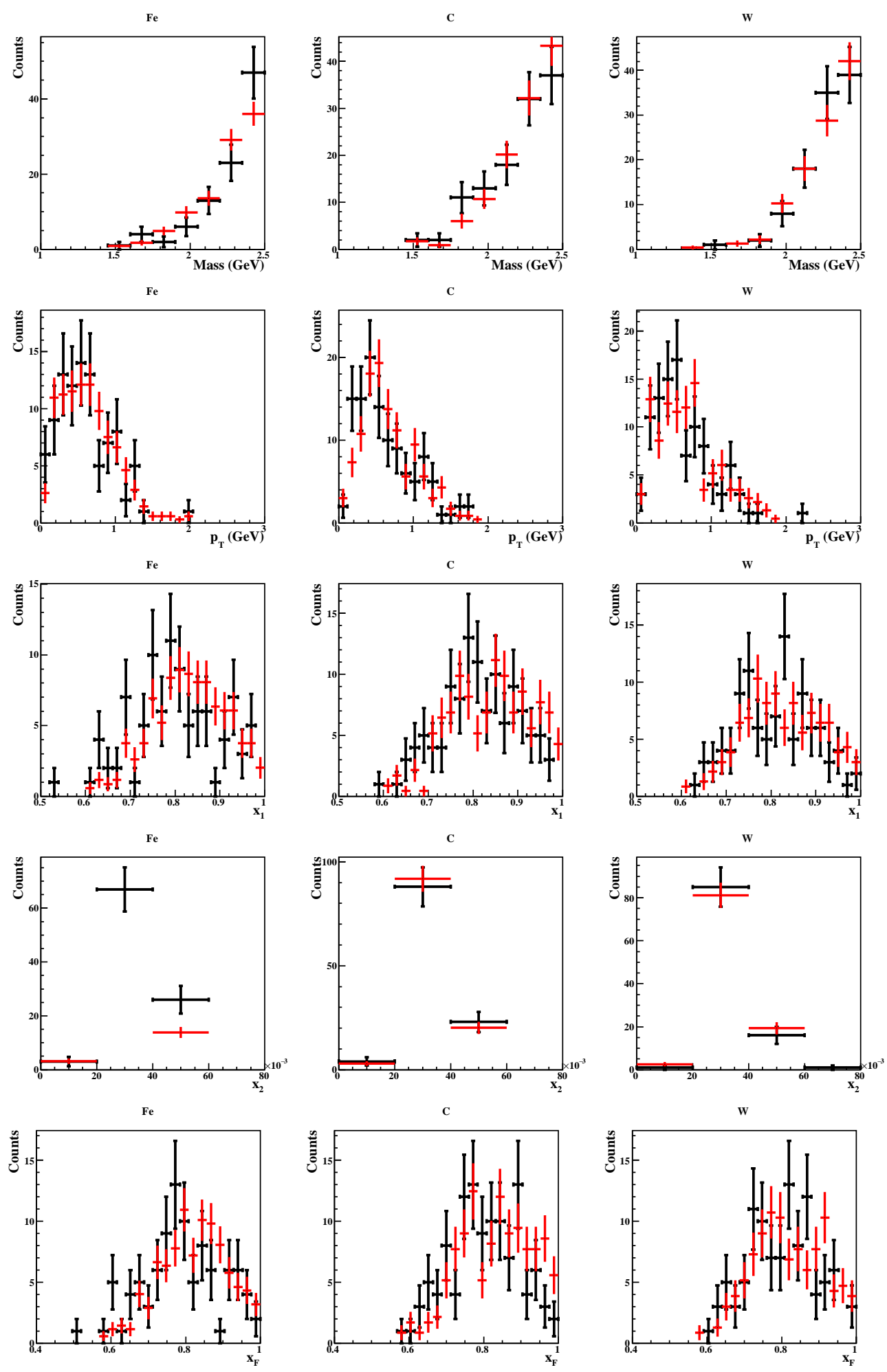

Figure 3.11: Comparison of distributions of kinematic variables of mass $<2.5 \mathrm{GeV}$ of Roadset 62 data. The variables are mass, $p_{T}, x_{1}, x_{2}$, and $x_{F}$, from top to bottom. The black points are the real data, and the red points are the random-mix data scaled to the same normalization. 
$\mathrm{Fe}$

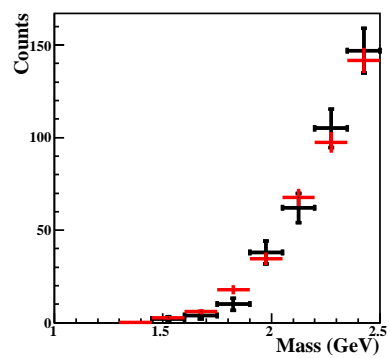

$\mathrm{Fe}$
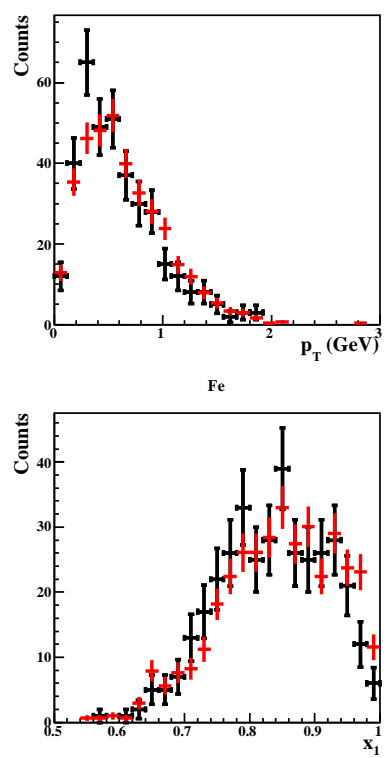

$\mathrm{Fe}$
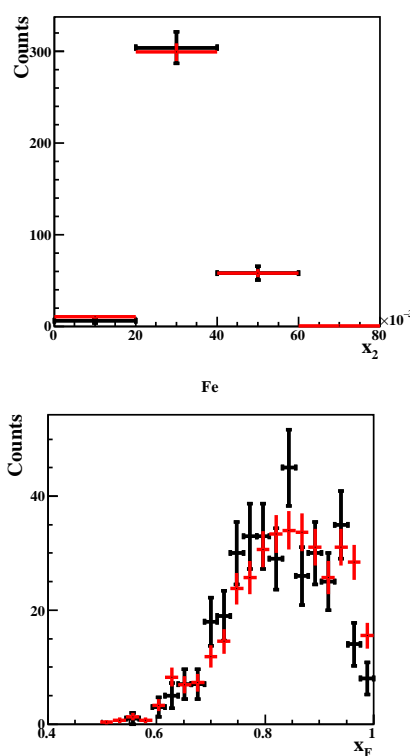

c
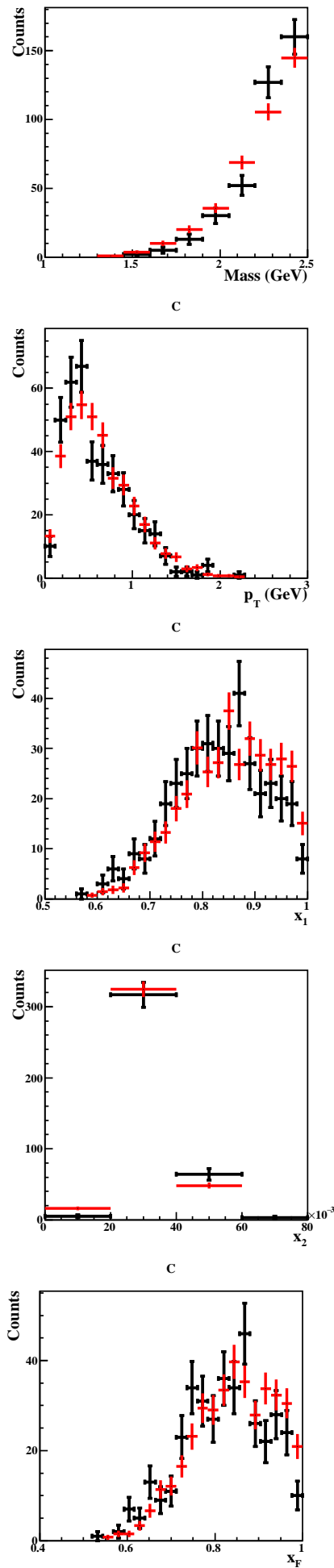

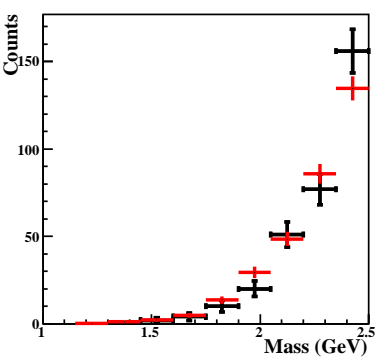

w
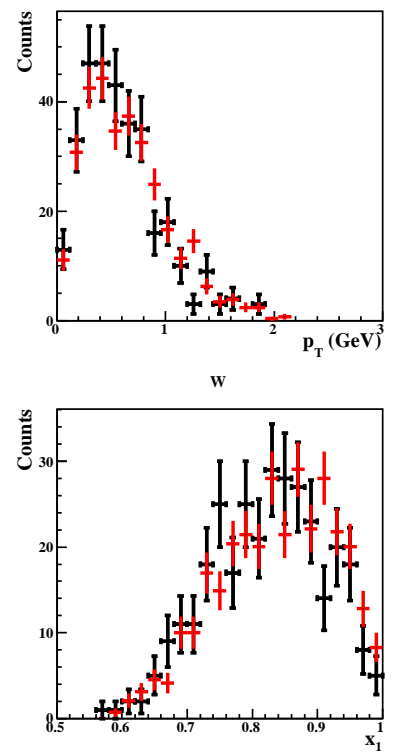

W
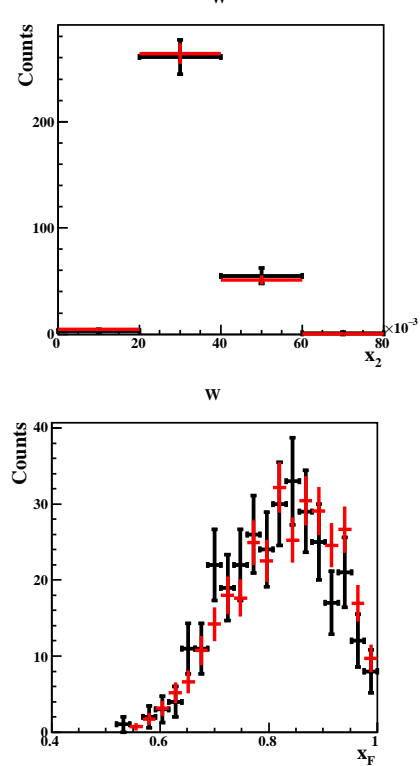

Figure 3.12: Comparison of distributions of kinematic variables of mass $<2.5 \mathrm{GeV}$ of Roadset 67 data. The variables are mass, $p_{T}, x_{1}, x_{2}$, and $x_{F}$, from top to bottom. The black points are the real data, and the red points are the random-mix data scaled to the same normalization. 


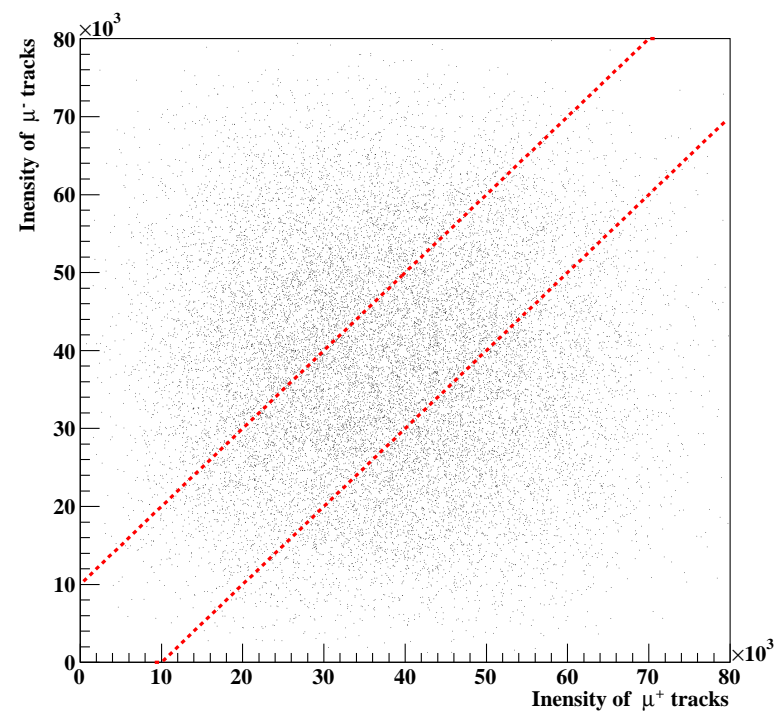

Figure 3.13: Scatter plot of the intensity of $\mu^{-}$tracks versus the intensity of $\mu^{+}$tracks from the Roadset 67 random-mix data.

from $\psi^{\prime}$ while keeping as many Drell-Yan events at the same time. Since we only checked the distribution of mass $<2.5 \mathrm{GeV}$, we need to be sure that the random-mix data still works in the high-mass region. Therefore, another factor, which is related to the beam-intensity dependence, that could affect the mass distribution of the random-mix data is also examined.

As we know, the hit rate of the detectors is correlated with position: the outer edge in the $\mathrm{X}$ direction would have higher hit-rate compared to the center. Since the detector performance would be affected by the hit rate, possible bias on the kinematics can be brought to the single tracks from different beam-intensity environment. To overcome this, the track mixing for producing the random-mix data should only combine tracks from events of similar beam intensity. On the other hand, this "correct-intensity mixing" approach would reduce the number of random-mixing events available, which is not preferred for the already limited data size at hand. It is therefore necessary to check if there is noticeable difference on the mass distribution between the simple mixing data and the correct-intensity mixing data.

Fig. 3.13 shows the intensities of the events corresponding to the $\mu^{+}$tracks versus those of 

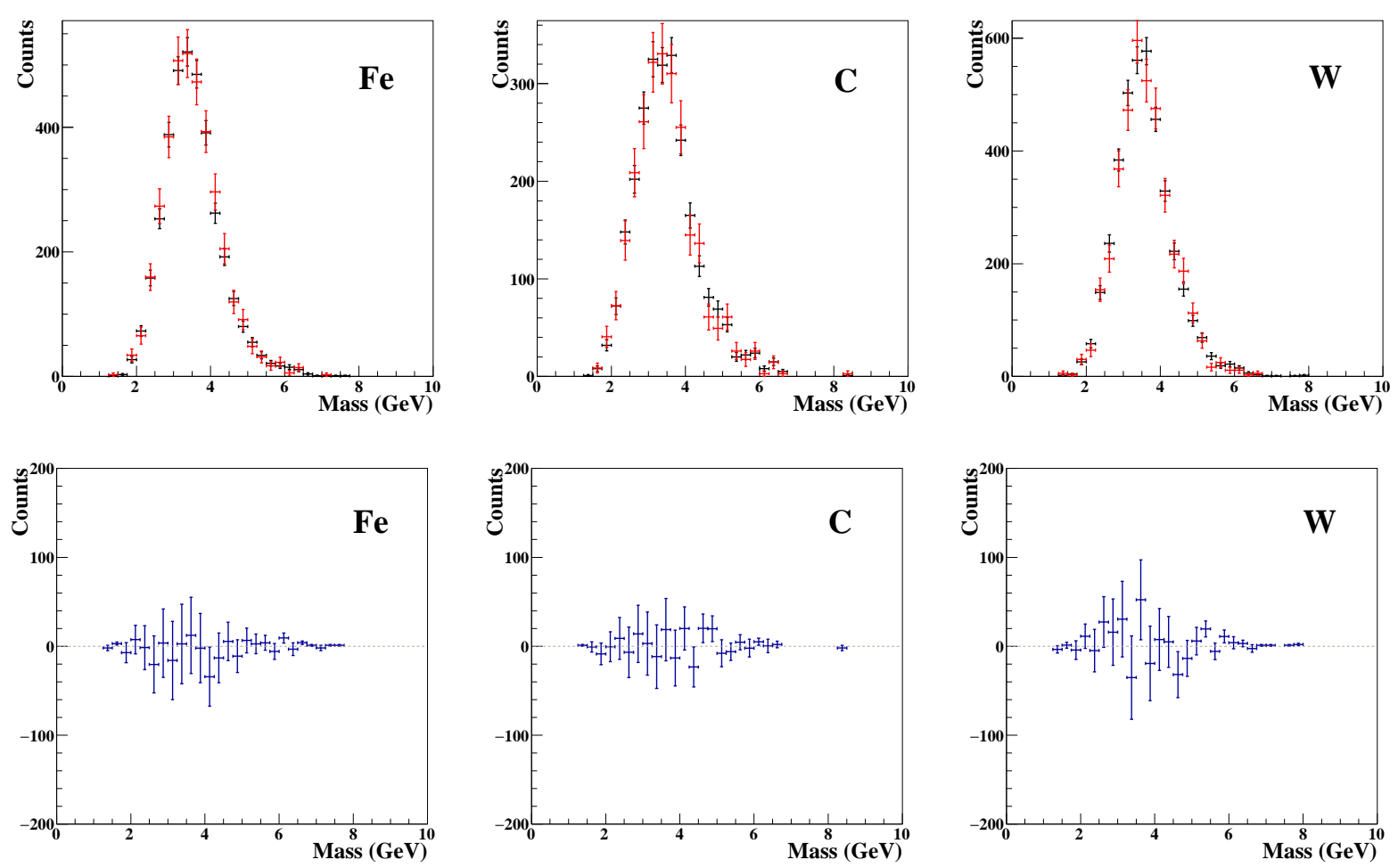

Figure 3.14: The Comparison of mass distributions between the correct-intensity-mix data and the whole random mix data. The plots in the top row show the mass distributoins of the two data respectively, where the correct-intensity-mix data is represented by the red points and the whole random-mix data is represented by the black points. The plots in the bottom row are the difference between the two mass spectra.

the $\mu^{-}$tracks of the random-mix data. As can be seen in the plot, there is no correlation between the intensities of the randomly paired $\mu^{+}$tracks and the $\mu^{-}$tracks. The correct-intensity-mix range is defined as the area between the two dashed lines shown in the plot, which requires the intensity difference between the tracks to be less than 10,000. The mass distributions of the correctintensity-mix events from different targets are then compared to the ones of the whole random-mix data set.

The comparison is given in Fig. 3.14, in which the two mass spectra of each target are plotted with same normalization of mass $<2.5 \mathrm{GeV}$, where the background events dominates. The result shows no significant deviation with mass $>2.5 \mathrm{GeV}$ and good agreement between the correct- 
intensity-mix data and the whole random-mix data for each of the target is confirmed. The beam intensity dependence effect on the track combination is therefore neglected and all the random-mix data will be used for representing the background. Now we are confident with the ability to describe the kinematic distribution of the background with the random-mixing method, the next step is to correctly determine the background numbers in different $x_{F}$ bins.

\subsubsection{Background Normalization}

To obtain the number of the background, two different approaches have been attempted and the results from these two methods will be cross checked to confirm that our understanding and the treatment of the background is on the right track. The first approach uses the like-sign muon pairs collected by the FPGA-3 trigger to give a statistical estimation. An example of the application of this method can be found in [114] and the derivation is given below. Consider the positive likesign muon pairs first. For an event in which $M^{+}$positive mesons are produced, the corresponding number of possible muon pairs is

$$
C_{2}^{M^{+}}=\frac{M^{+} !}{2\left(M^{+}-2\right) !}=\frac{M^{+^{2}}-M^{+}}{2} .
$$

In different events, the numbers of those muon-producing mesons generated should follow the Poisson statistics. Let $P\left(M^{+}\right)$be the probability of generating $M^{+}$mesons and $f$ the probability for a meson to decay into final states with $\mu^{+}$, the number of the positive like-sign muon pairs, $N^{++}$, formed within $N$ interactions is

$$
N^{++}=A^{++} \int N P\left(M^{+}\right) \frac{M^{+2}-M^{+}}{2} f^{2} d M^{+}=A^{++} N f^{2} \frac{\left\langle M^{+2}\right\rangle-\left\langle M^{+}\right\rangle}{2} .
$$

According to the Poisson statistics, $\left\langle M^{+}\right\rangle=\left\langle M^{+^{2}}\right\rangle-\left\langle M^{+}\right\rangle^{2}$. Bring this into Eq. 3.7 and it becomes

$$
N^{++}=A^{++} N f^{2} \frac{\left\langle M^{+}\right\rangle^{2}}{2}
$$

where $A^{++}$represents the geometric acceptance for the $\mu^{+}$pairs. Similarly, the number of negative like-sign muon pairs can be derived as:

$$
N^{--}=A^{--} N f^{2} \frac{\left\langle M^{-}\right\rangle^{2}}{2}
$$


Finally, following the same logic, the number of the combinatorial background can be derived

as

$$
\begin{aligned}
N^{+-} & =A^{+-} \int N\left[P\left(M^{+}\right) M^{+} f d M^{+}\right]\left[P\left(M^{-}\right) M^{-} f d M^{-}\right] \\
& =A^{+-} N f^{2}\left\langle M^{+}\right\rangle\left\langle M^{-}\right\rangle .
\end{aligned}
$$

From Eq. 3.8 and 3.9 it is easily seen that:

$$
\left\langle M^{+}\right\rangle=\sqrt{\frac{2 N^{++}}{A^{++} N f^{2}}},\left\langle M^{-}\right\rangle=\sqrt{\frac{2 N^{--}}{A^{--} N f^{2}}} .
$$

Bring these into Eq. 3.10, we have

$$
N^{+-}=2 \sqrt{N^{++} N^{--}} \frac{A^{+-}}{\sqrt{A^{++} A^{--}}}
$$

Since the SeaQuest spectrometer and trigger is designed in the way that the $\mu^{+} s$ and $\mu^{-} s$ are detected uncorrelatedly before being combined to form dimuon pairs, the acceptance in the equations can simply be written as the product of the acceptance of $\mu^{+}$and $\mu^{-}$, like $A^{+-}=A^{+} A^{-}$for example. Eq 3.11 can thus be simplified as

$$
N^{+-}=2 \sqrt{N^{++} N^{--}}
$$

with the error

$$
\delta N^{+-}=N^{+-} \frac{\sqrt{N^{++}}+\sqrt{N^{--}}}{2 \sqrt{N^{++} N^{--}}} .
$$

Using the like-sign trigger (FPGA-3) data, apply the same cuts like the FPGA-1 events but require both the muon tracks to have the same sign of charge, and the like sign event count, $N^{++}$ and $N^{--}$, can be obtained. As given in Tab. 2.6. since the prescale factor of 123 is applied to the FPGA-3 trigger, the number $N^{+-}$acquired by Eq. 3.12 must be multiplied by 123 to give the correct number of backgrounds. The estimated background number and uncertainty is summarized in Tab. 3.12,

From Tab. 3.12 we can see that $N^{++}$is greater than $N^{--}$in general, which is reasonable since it is the proton beam that is used to interact with the target and more positive random muons 


\begin{tabular}{ccccc}
\hline Data set & Target & $N^{++}$ & $N^{--}$ & $N^{+-} \times 123$ \\
\hline Run-II & Fe & 8 & 1 & $696 \pm 471$ \\
& $\mathrm{C}$ & 4 & 6 & $1205 \pm 435$ \\
& $\mathrm{~W}$ & 4 & 1 & $492 \pm 369$ \\
\hline Roadset 62 & $\mathrm{Fe}$ & 6 & 1 & $603 \pm 424$ \\
& $\mathrm{C}$ & 4 & 1 & $492 \pm 277$ \\
& $\mathrm{~W}$ & 4 & 8 & $1392 \pm 594$ \\
\hline Roadset 67 & $\mathrm{Fe}$ & 23 & 11 & $3913 \pm 998$ \\
& $\mathrm{C}$ & 20 & 8 & $3112 \pm 683$ \\
& $\mathrm{~W}$ & 23 & 8 & $3337 \pm 938$ \\
\hline
\end{tabular}

Table 3.12: Estimation of the background number using the FPGA-3 event. The 123 used to mutiply $N^{+-}$is the prescale factor of FPGA-3 trigger.

would be thus generated due to charge conservation. However, some problems with this method using like-sign muon pairs can be immediately observed from the results. First, due to the fact that the FPFA-3 trigger is prescaled by a factor of 123, the resulting error of the background number is large and can be as as big as more than $25 \%$ of the estimated background. The more serious issue is that the number of the like-sign events is already low in the beginning, which is what we expect for the cuts optimized for selecting true dimuon events, and it is made even worse by the prescale setting. As shown in Tab. 3.12, for small data sets, Run-II and Roadset 62, there are cases which have only one single like-sign event in it. With these extremely limited statistics and their possible significant fluctuation, to estimate the background number based on the like-sign event number would just fail and not trustworthy for these two data sets. A different measure needs to be take.

The second method is to determine the background normalization by fitting of the mass distribution. In principle, the combinatorial background in different $x_{F}$ bins can be extracted out by fitting the mass spectrum using the Monte Carlo simulated Drell-Yan, $J / \psi, \psi^{\prime}$, and the randommix events since the validity of the mass distribution of the random-mix data has been confirmed. However, as illustrated in Fig. 3.15, the mass of random-mix events is dependent to $x_{F}$ and tends to be distributed at higher mass with decreasing $x_{F}$. In the low $x_{F}$ region, the mass spectrum of the Drell-Yan and the random-mix events become largely overlapped and similar to each other. 

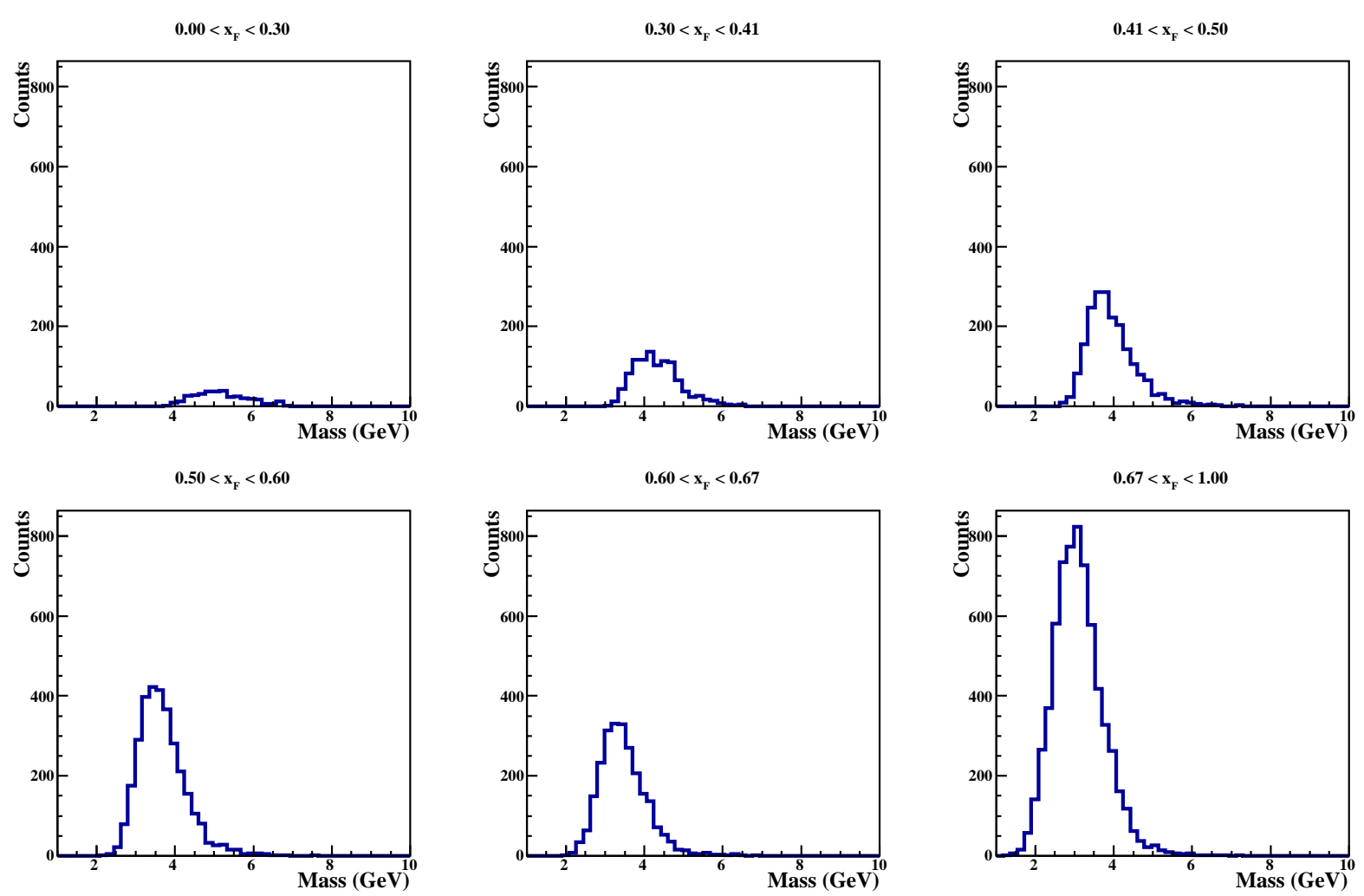

Figure 3.15: Mass distributions of Roadset 67 random-mix iron target events in nominal $x_{F}$ bins. All the plots are made with same scale in order to show in order to show the relative contribution in different $x_{F}$ bin.

This makes the mass spectrum fitting in low $x_{F}$ unstable and therefore unreliable. Nevertheless, we can try to make use of the fitting result in the high $x_{F}$ bin, in which the background number can be unambiguously determined, and extrapolate the normalization factor acquired to the low $x_{F}$ region to acquire the background number there.

For example, if the fitting in the high $x_{F}$ bin, $B_{1}$, gives the number of background to be $N_{f i t}\left(B_{1}\right)$, we can then divide $N_{f i t}\left(B_{1}\right)$ by the number of random-mix events in this $x_{F}$ bin, $N_{m i x}\left(B_{1}\right)$, to obtain a normalization factor for each of the random-mix event,

$$
F_{b k g}=N_{f i t}\left(B_{1}\right) / N_{m i x}\left(B_{1}\right)
$$

Then for a low $x_{F}$ bin, $B_{2}$, to retrieve the background number $N_{b k g}\left(B_{2}\right)$ we simply multiply the 
random-mix event number in that bin and get

$$
N_{b k g}\left(B_{2}\right)=F_{b k g} \cdot N_{m i x}\left(B_{2}\right) .
$$

Clearly, it is assumed that the normalization factor $F_{b k g}$ doesn't depend on $x_{F}$ for this method to work. Nevertheless, this would be a solid assumption if the background is represented well by the random-mix events in the $x_{F}$ phase space and there is no significant variation caused by the $x_{F}$ binning. In this case, $F_{b k g}$ is simply the ratio between two nearly similar $x_{F}$ distributions and shouldn't carry $x_{F}$ dependence in it.

The consistency between the random-mix and the background $x_{F}$ distribution has already been tested in Section 3.6 .2 . We would further verify this by extracting the $F_{b k g}$ values from different $x_{F}$ bins in the high $x_{F}$ region and examine the agreement between them. The $x_{F}$ range is chosen to be from 0.63 to 1.00 so that the random-mix data in the lowest $x_{F}$ bin would still has decent contribution from the low mass part that can be used to differentiate the random-mix distribution from the distributions of other sources and therefore keep the correlation between them low. This $x_{F}$ range is divided into three sub-bins to keep an acceptable number of real events in each bin for fitting. One thing that need to be mentioned is that while performing the mass fitting, Gaussian distributions were used to represent the mass distribution of $J / \psi$ and $\psi^{\prime}$, rather than using the Monte Carlo generated ones. The reason is that it is observed that the MC $J / \psi$ mass width is slightly wider than the real-data mass peak at the $J / \psi$ mass, and therefore cause the $J / \psi$ mass peak not well described by the fitting and would lead to over-estimating the background contribution. Since the $\mathrm{MC} J / \psi$ and $\psi^{\prime}$ mass would depend on the energy-loss parameterization of the muon tracks which needs to be further calibrated, it is decided to use Gaussian distribution instead. To provide further constraint in fitting, the mass distributions of the solid targets are fitted simultaneously, by using the same Gaussian distribution representing $J / \psi$ and also $\psi^{\prime}$ for each target. Rather than being a free parameter in fitting, the Gaussian center of $\psi^{\prime}$ is set as 3.686/3.097 of the center of $J / \psi$, since the energy-loss effect shouldn't affect the mass-peak ratio between $J / \psi$ and $\psi^{\prime}$. The fitting results are also compared with the ones using the MC $J / \psi$ and 
$\psi^{\prime}$ mass, and they all agree within error.

The mass fittings of each target of each data set are shown in Fig. 3.16, 3.18, and 3.20. We can see from these figures that the mass distributions are well depicted by the fitting results, which serves as another indirect confirmation of the successful description of background of mass greater than $2.5 \mathrm{GeV}$. The fitting extracted $F_{b k g}$ values are shown in Fig 3.17, 3.19, and 3.21, together with the $F_{b k g}$ acquired by the fitting of the highest nominal $x_{F}$ bin, $0.67<x_{F}<1.00$. $F_{b k g}$ factors can also be evaluated for the like-sign-event estimated background by dividing the background numbers given in Tab. 3.12 by the corresponding random-mix events, and the values together with their errors are also shown in the figures as the light blue bands. First of all, with non-drastic fluctuation in some cases, the $F_{b k g}$ value in different $x_{F}$ ranges agrees with each other within 1- $\sigma$ in general. This results assured us even more that it is appropriate to assume that $F_{b k g}$ is not $x_{F}$ dependent. Secondly, by comparing the results from the like like-sign-event estimation from the ones from fitting, it can be observed that although bearing the large errors, the values from the like-sign-event method are smaller than the fitting ones. This is, however, expectable due to the low-statistic nature of the like-sign events that can survive. If we assume the numbers we have got are correct and use the ratio between the numbers from fitting and from the like-sign-event estimation, the most limited factor, $N^{--}$, would only be expected to be on the order of 10 even for the largest Roadset 67 data. Based on Poisson statistics, the measurement would more likely render numbers smaller than the expectation value, which is what we observed. 

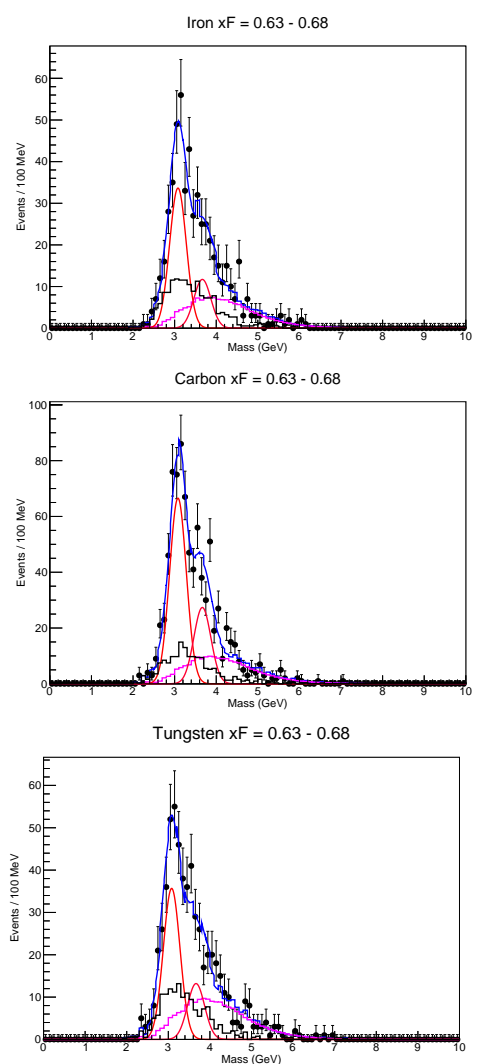

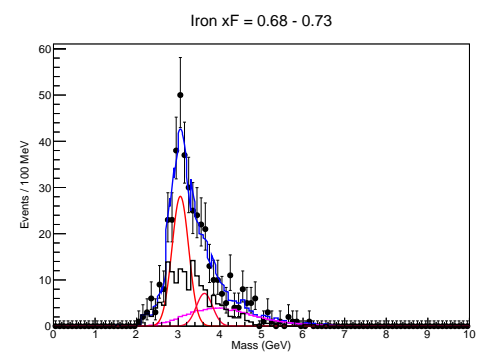

Carbon $\mathrm{xF}=0.68-0.73$
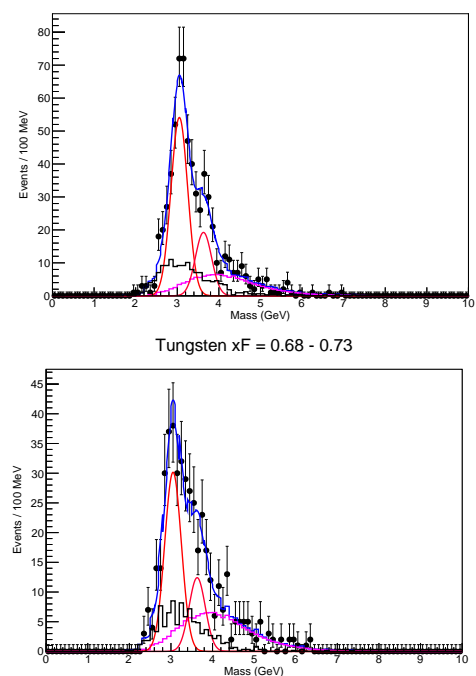

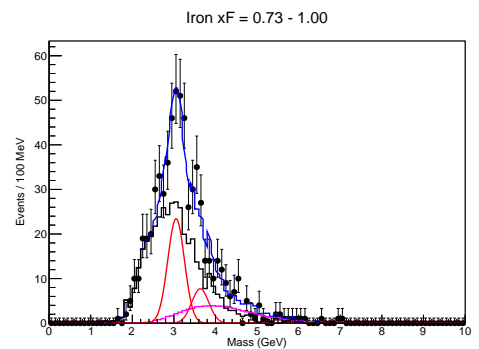

Carbon $\mathrm{xF}=0.73-1.00$
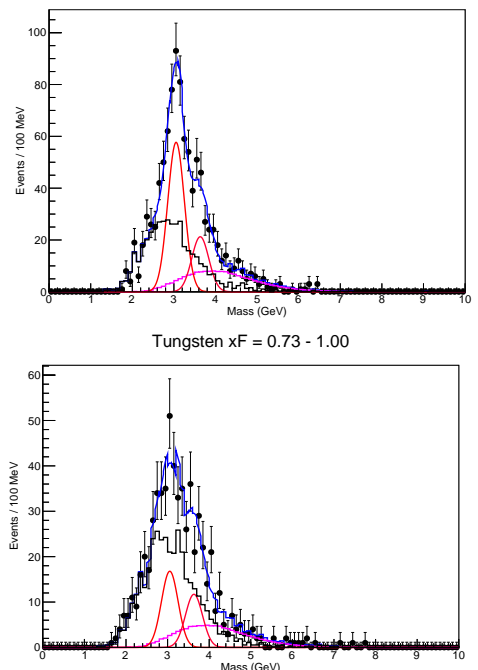

Figure 3.16: Mass fitting of the Run-II data in different $x_{F}$ bins. The magenta line is the Drell-Yan Monte Carlo events. The red lines are $J / \psi$ and $\psi^{\prime}$. The black line is the combinatorial background. The sum of all the sources is represented by the blue line.

$\mathrm{Fe}$

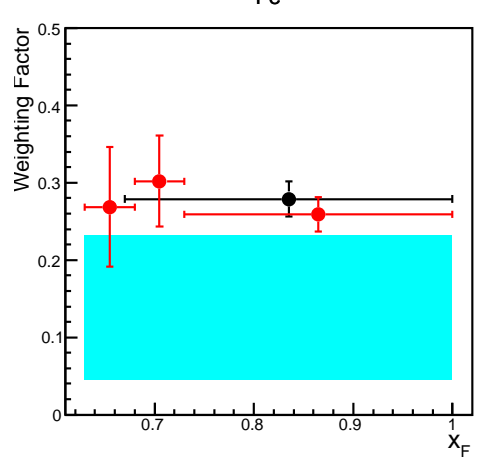

C

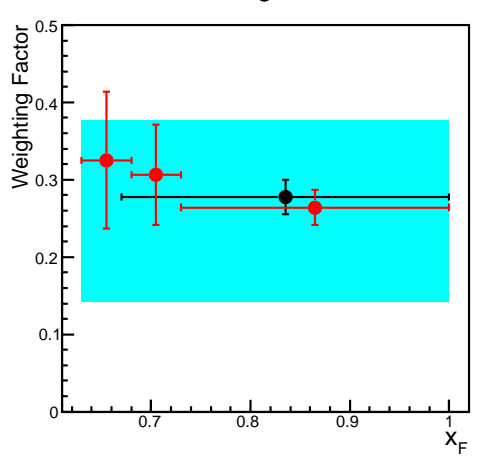

W

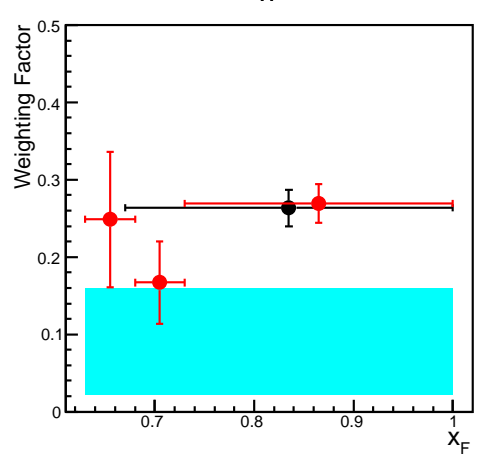

Figure 3.17: $F_{b k g}$ of the Run-II data in different $x_{F}$ bins. The black point shows the value acquired by the highest nominal $x_{F}$ bin and the red points are the values acquired with finer binning. The result derived from the like-sign-event estimation is given as the ligh-blue band. 

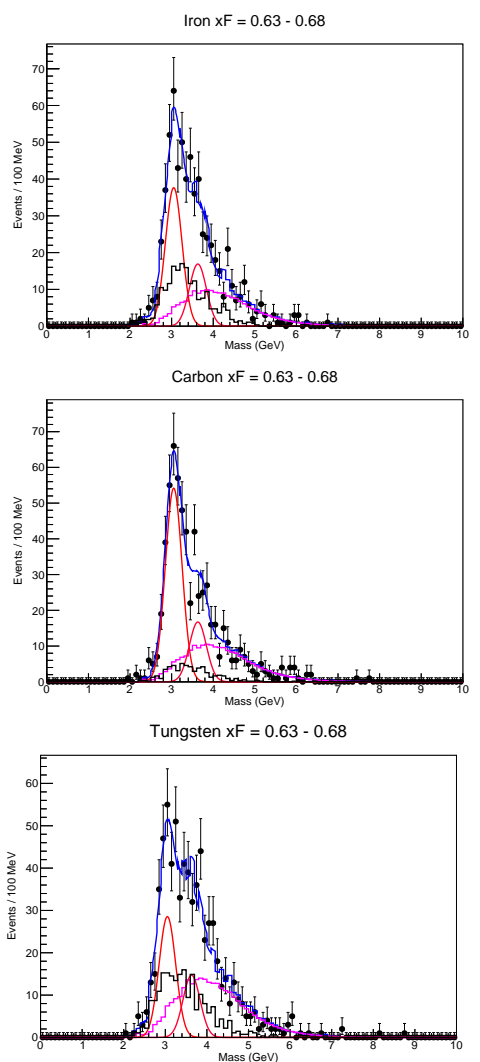

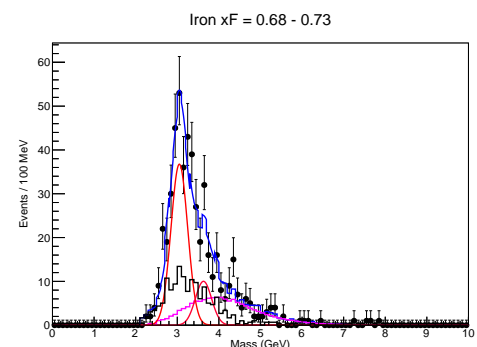

Carbon $x F=0.68-0.73$
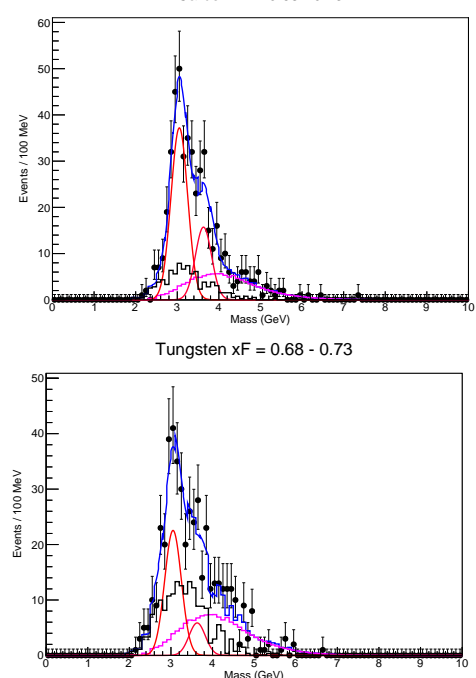

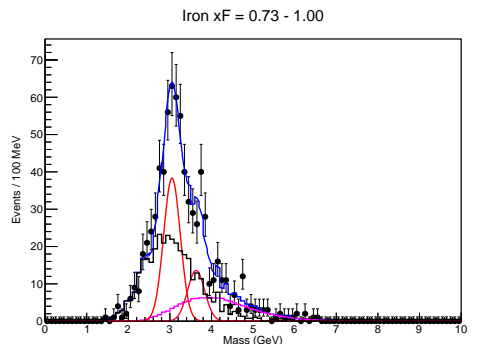

Carbon $x F=0.73-1.00$

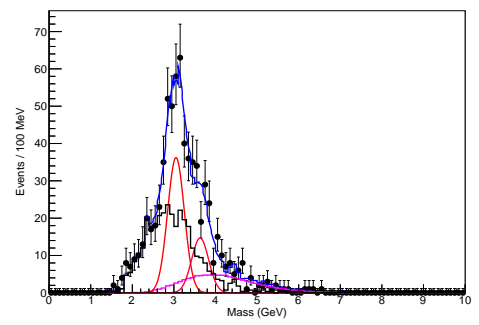

Tungsten $x F=0.73-1.00$

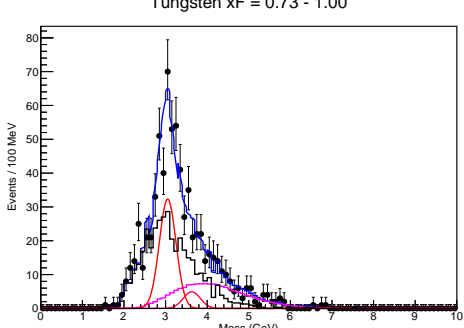

Figure 3.18: Mass fitting of the Roadset 62 data in different $x_{F}$ bins. The magenta line is the Drell-Yan Monte Carlo events. The red lines are $J / \psi$ and $\psi^{\prime}$. The black line is the combinatorial background. The sum of all the sources is represented by the blue line.

$\mathrm{Fe}$

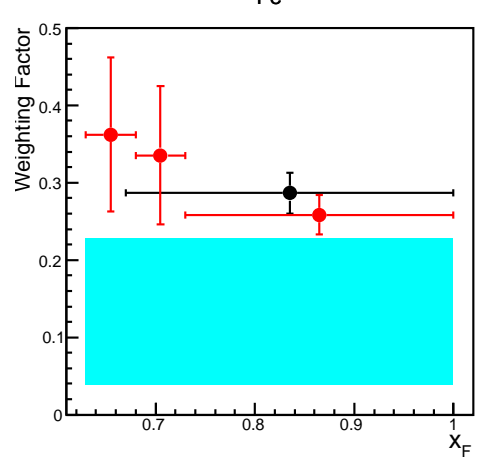

C

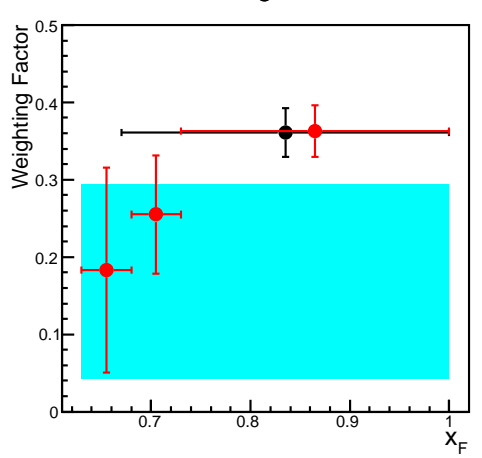

W

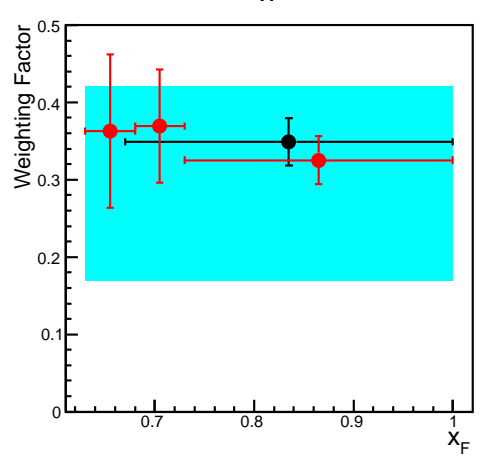

Figure 3.19: $F_{b k g}$ of the Roadset 62 data in different $x_{F}$ bins. The black point shows the value acquired by the highest nominal $x_{F}$ bin and the red points are the values acquired with finer binning. The result derived from the like-sign-event estimation is given as the ligh-blue band. 


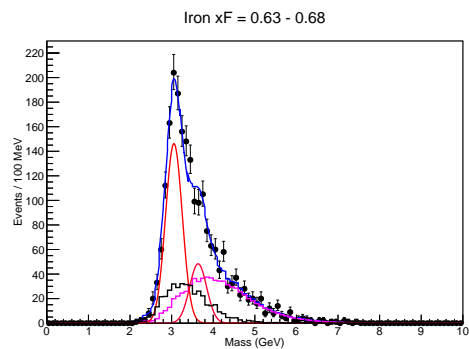

Carbon $x F=0.63-0.68$

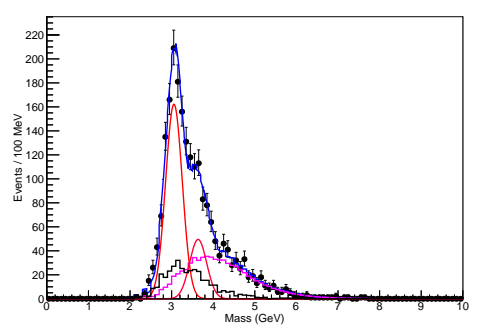

Tungsten $\mathrm{xF}=0.63-0.68$

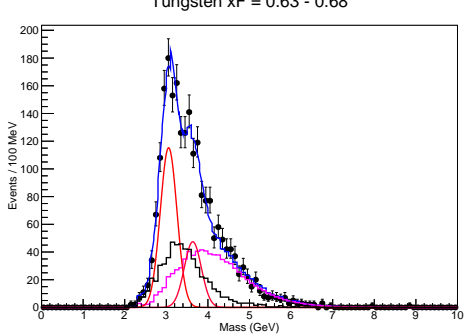

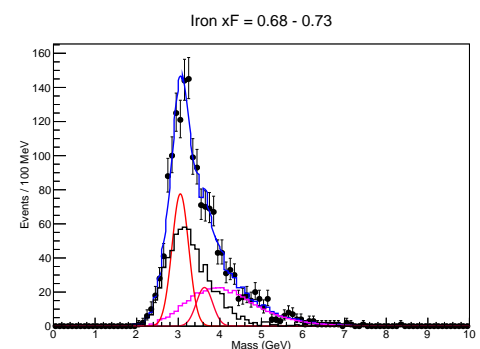

Carbon $x F=0.68-0.73$

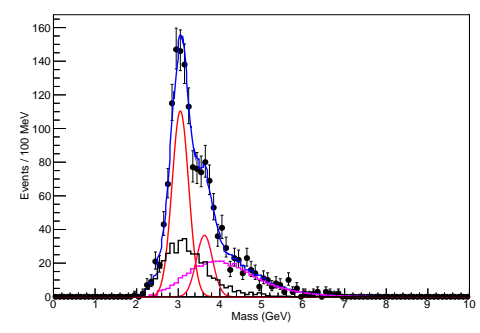

Tungsten $\mathrm{xF}=0.68-0.73$

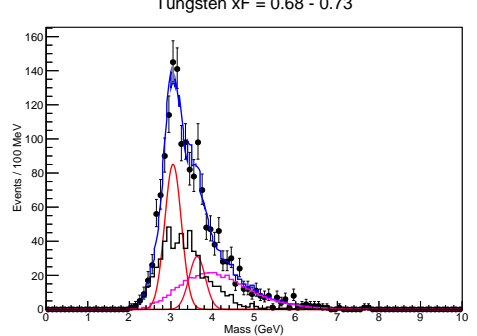

Iron $x F=0.73-1.00$

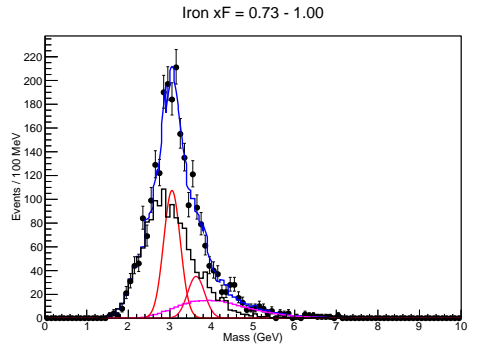

Carbon $x F=0.73-1.00$

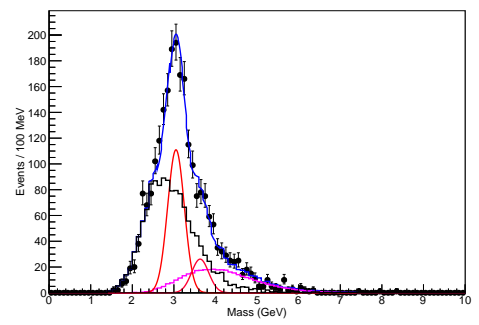

Tungsten $\mathrm{xF}=0.73-1.00$

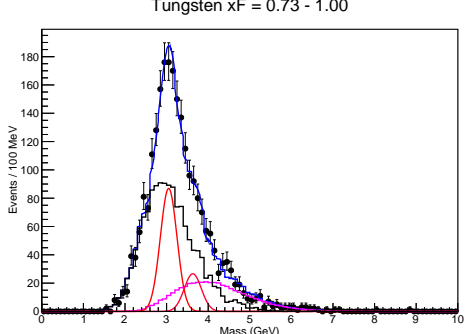

Figure 3.20: Mass fitting of the Roadset 67 data in different $x_{F}$ bins. The magenta line is the Drell-Yan Monte Carlo events. The red lines are $J / \psi$ and $\psi^{\prime}$. The black line is the combinatorial background. The sum of all the sources is represented by the blue line.

$\mathrm{Fe}$

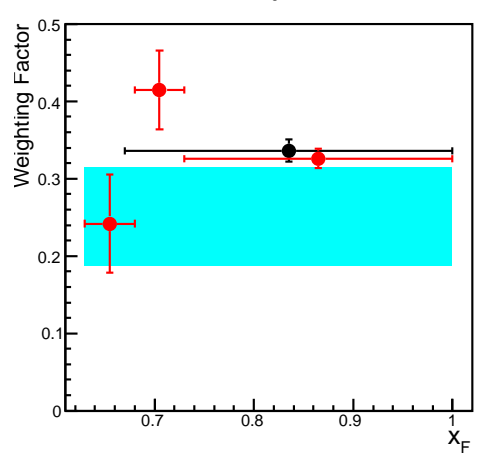

C

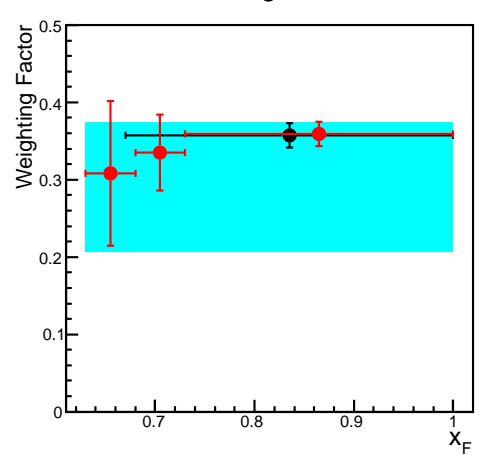

W

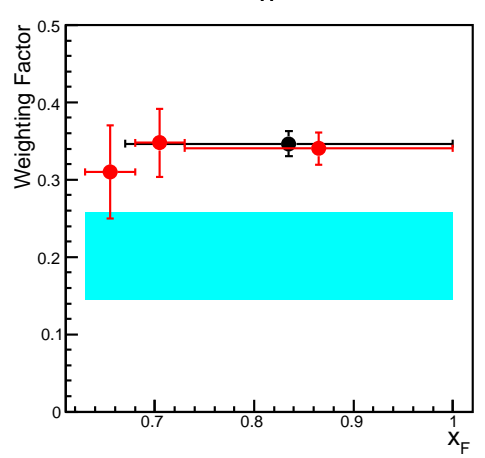

Figure 3.21: $F_{b k g}$ of the Roadset 67 data in different $x_{F}$ bins. The black point shows the value acquired by the highest nominal $x_{F}$ bin and the red points are the values acquired with finer binning. The result derived from the like-sign-event estimation is given as the ligh-blue band. 


\begin{tabular}{ccc}
\hline Data set & Target & $F_{b k g}$ \\
\hline Run-II & Fe & $0.2789 \pm 0.0223$ \\
& $\mathrm{C}$ & $0.2776 \pm 0.0219$ \\
& $\mathrm{~W}$ & $0.2636 \pm 0.0236$ \\
\hline Roadset 62 & Fe & $0.2865 \pm 0.0262$ \\
& $\mathrm{C}$ & $0.3607 \pm 0.0316$ \\
& $\mathrm{~W}$ & $0.3490 \pm 0.0305$ \\
\hline Roadset 67 & Fe & $0.3362 \pm 0.0145$ \\
& $\mathrm{C}$ & $0.3571 \pm 0.0158$ \\
& $\mathrm{~W}$ & $0.3466 \pm 0.0162$ \\
\hline
\end{tabular}

Table 3.13: List of $F_{b k g}$ values acquired in the highest nominal $x_{F}$ bin, $0.67<x_{F}<1.00$.

The SeaQuest collaboration has notice the low-statistics problem of the like-sign data and the FPGA-3 prescale value been set at 11 starting from Run-IV, which is more than 10 times smaller than the previous setting. With expected higher number of fPGA-3 events, more accurate cross check can be performed in the upcoming data sets. At this point, it is rightful to claim that a coherent picture is seen in the combinatorial background treatment. For the evaluation of the background number, the $F_{b k g}$ values derived by mass fitting from the highest nominal $x_{F}$ bin are used, as summarized in Tab. 3.13. In this way, most of the low mass tail of the background is used so the $F_{b k g}$ can be most accurately determined. The $F_{b k g}$ value is then applied as a weighting for the random-mix events in each $x_{F}$ bin and the number of background is acquired. The fitting error of $F_{b k g}$ is used as a contribution to the systematic error of $R_{p A}$ and will be denoted as $\sigma_{s y s}^{B G f i t}$ in the discussion later. With mass $>4.2 \mathrm{GeV}$ being required, the background subtraction is illustrated in Fig. 3.22, 3.23, and 3.24 The number of the raw yield $N^{\text {raw }}$, the estimated background $N^{b k g}$, and the background-subtracted yield $Y^{D Y}$, are summarized in Tab. 3.14, 3.15, and 3.16. A consistency check of kinematic variable distributions between different data sets after background subtraction is given in Fig. 3.25, and the general agreement among all data sets can be seen. The average values of the kinematic variables are listed in Tab. 3.17, 3.18, and 3.19, Again, good consistency between data sets can be observed in each observable, each $x_{F}$ bin. 

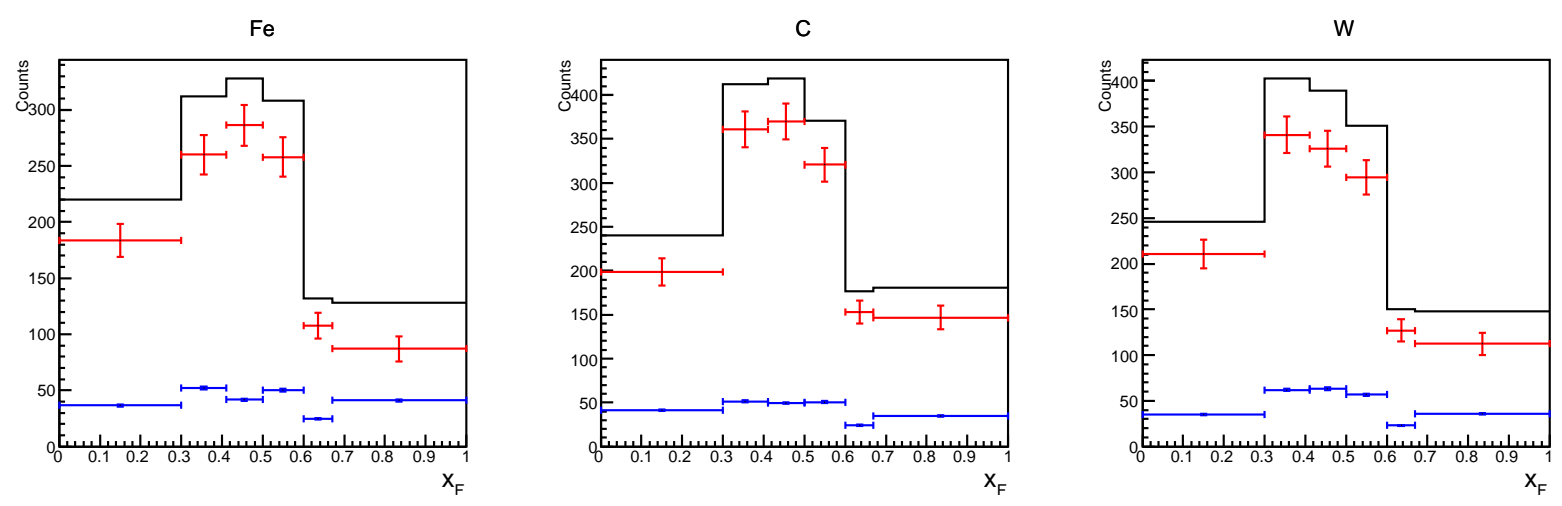

Figure 3.22: Illustration of background subtraction in nominal $x_{F}$ bins of Run-II data. The black histogram represents $N^{r a w}$, the blue points are $N^{b k g}$, and the red points are $Y^{D Y}$.

\begin{tabular}{cccccc}
\hline Target & $x_{F}$ bin & $N^{\text {raw }}$ & $N^{\text {bkg }}$ & $N^{\text {bkg }} / N^{\text {raw }}(\%)$ & $Y^{D Y}$ \\
\hline Fe & $0.00<x_{F} \leq 0.30$ & 220 & 37 & 17 & 183 \\
& $0.30<x_{F} \leq 0.41$ & 312 & 52 & 17 & 260 \\
& $0.41<x_{F} \leq 0.50$ & 328 & 42 & 13 & 286 \\
& $0.50<x_{F} \leq 0.60$ & 308 & 50 & 16 & 258 \\
& $0.60<x_{F} \leq 0.67$ & 132 & 25 & 19 & 107 \\
& $0.67<x_{F} \leq 1.00$ & 128 & 41 & 32 & 87 \\
\hline C & $0.00<x_{F} \leq 0.30$ & 240 & 41 & 17 & 199 \\
& $0.30<x_{F} \leq 0.41$ & 412 & 51 & 12 & 361 \\
& $0.41<x_{F} \leq 0.50$ & 419 & 49 & 12 & 370 \\
& $0.50<x_{F} \leq 0.60$ & 371 & 51 & 14 & 320 \\
& $0.60<x_{F} \leq 0.67$ & 177 & 24 & 14 & 153 \\
& $0.67<x_{F} \leq 1.00$ & 181 & 34 & 19 & 147 \\
\hline $\mathrm{W}$ & $0.00<x_{F} \leq 0.30$ & 246 & 35 & 14 & 211 \\
& $0.30<x_{F} \leq 0.41$ & 403 & 62 & 15 & 341 \\
& $0.41<x_{F} \leq 0.50$ & 389 & 63 & 16 & 326 \\
& $0.50<x_{F} \leq 0.60$ & 351 & 57 & 16 & 294 \\
& $0.60<x_{F} \leq 0.67$ & 150 & 23 & 15 & 127 \\
& $0.67<x_{F} \leq 1.00$ & 148 & 36 & 24 & 112 \\
\hline
\end{tabular}

Table 3.14: Summary of background subtraction of Run-II data. 

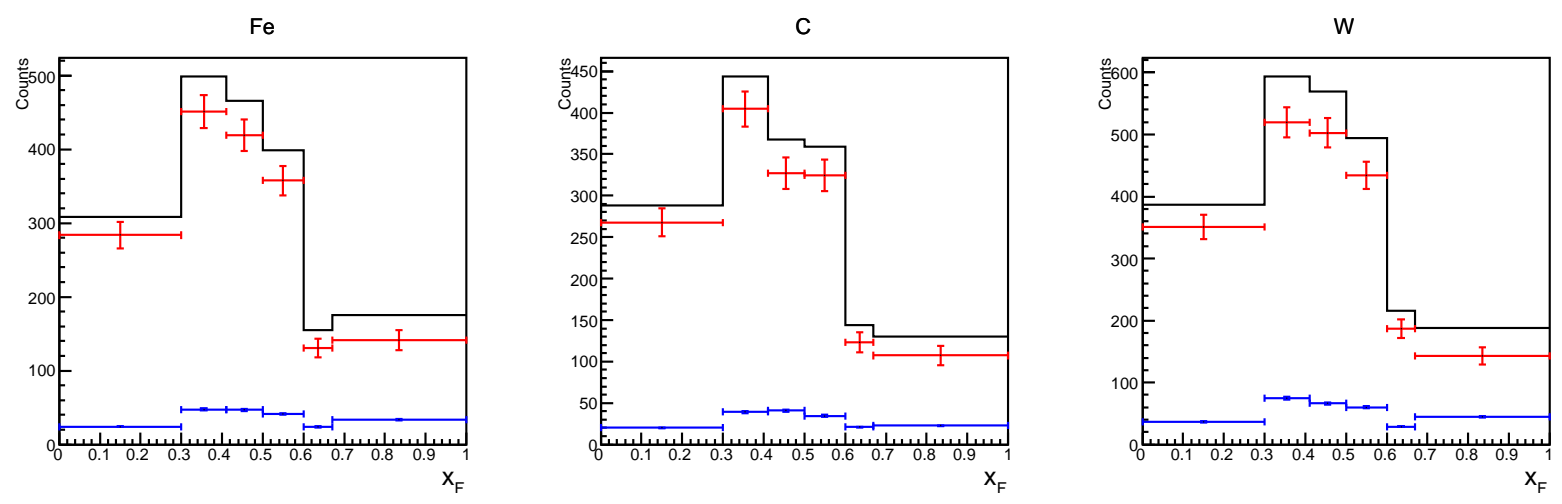

Figure 3.23: Illustration of background subtraction in nominal $x_{F}$ bins of Roadset 62 data. The black histogram represents $N^{\text {raw }}$, the blue points are $N^{b k g}$, and the red points are $Y^{D Y}$.

\begin{tabular}{cccccc}
\hline Target & $x_{F}$ bin & $N^{\text {raw }}$ & $N^{\text {bkg }}$ & $N^{\text {bkg }} / N^{\text {raw }}(\%)$ & $Y^{D Y}$ \\
\hline Fe & $0.00<x_{F} \leq 0.30$ & 308 & 24 & 8 & 284 \\
& $0.30<x_{F} \leq 0.41$ & 499 & 48 & 10 & 451 \\
& $0.41<x_{F} \leq 0.50$ & 466 & 47 & 10 & 419 \\
& $0.50<x_{F} \leq 0.60$ & 399 & 42 & 10 & 357 \\
& $0.60<x_{F} \leq 0.67$ & 155 & 24 & 16 & 131 \\
& $0.67<x_{F} \leq 1.00$ & 175 & 34 & 19 & 141 \\
\hline $\mathrm{C}$ & $0.00<x_{F} \leq 0.30$ & 288 & 20 & 7 & 268 \\
& $0.30<x_{F} \leq 0.41$ & 444 & 39 & 9 & 405 \\
& $0.41<x_{F} \leq 0.50$ & 368 & 41 & 11 & 327 \\
& $0.50<x_{F} \leq 0.60$ & 359 & 35 & 10 & 324 \\
& $0.60<x_{F} \leq 0.67$ & 144 & 21 & 15 & 123 \\
& $0.67<x_{F} \leq 1.00$ & 130 & 23 & 17 & 107 \\
\hline $\mathrm{W}$ & $0.00<x_{F} \leq 0.30$ & 387 & 36 & 9 & 351 \\
& $0.30<x_{F} \leq 0.41$ & 594 & 75 & 13 & 519 \\
& $0.41<x_{F} \leq 0.50$ & 569 & 66 & 12 & 503 \\
& $0.50<x_{F} \leq 0.60$ & 494 & 60 & 12 & 434 \\
& $0.60<x_{F} \leq 0.67$ & 216 & 29 & 13 & 187 \\
& $0.67<x_{F} \leq 1.00$ & 188 & 45 & 24 & 143 \\
\hline
\end{tabular}

Table 3.15: Summary of background subtraction of Roadset 62 data. 

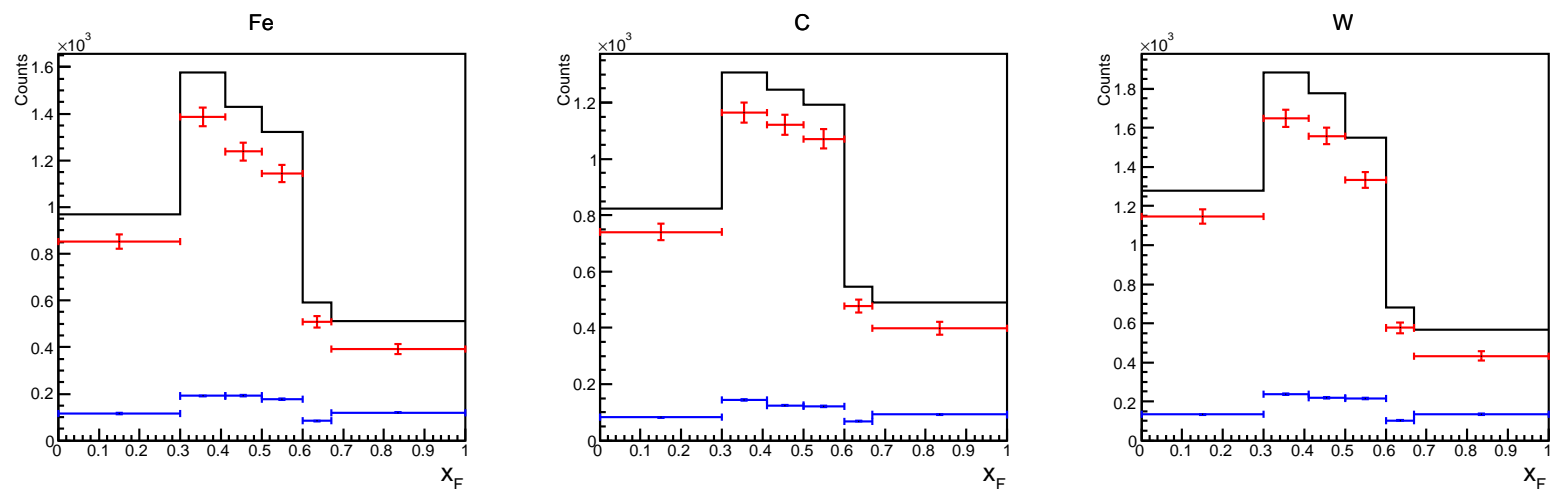

Figure 3.24: Illustration of background subtraction in nominal $x_{F}$ bins of Roadset 67 data. The black histogram represents $N^{\text {raw }}$, the blue points are $N^{b k g}$, and the red points are $Y^{D Y}$.

\begin{tabular}{cccccc}
\hline Target & $x_{F}$ bin & $N^{\text {raw }}$ & $N^{\text {bkg }}$ & $N^{\text {bkg }} / N^{\text {raw }}(\%)$ & $Y^{D Y}$ \\
\hline Fe & $0.00<x_{F} \leq 0.30$ & 969 & 116 & 12 & 853 \\
& $0.30<x_{F} \leq 0.41$ & 1578 & 191 & 12 & 1387 \\
& $0.41<x_{F} \leq 0.50$ & 1429 & 191 & 13 & 1238 \\
& $0.50<x_{F} \leq 0.60$ & 1321 & 178 & 13 & 1143 \\
& $0.60<x_{F} \leq 0.67$ & 592 & 84 & 14 & 508 \\
& $0.67<x_{F} \leq 1.00$ & 511 & 119 & 23 & 392 \\
\hline $\mathrm{C}$ & $0.00<x_{F} \leq 0.30$ & 823 & 82 & 10 & 741 \\
& $0.30<x_{F} \leq 0.41$ & 1308 & 144 & 11 & 1164 \\
& $0.41<x_{F} \leq 0.50$ & 1246 & 125 & 10 & 1121 \\
& $0.50<x_{F} \leq 0.60$ & 1193 & 122 & 10 & 1071 \\
& $0.60<x_{F} \leq 0.67$ & 545 & 68 & 13 & 477 \\
& $0.67<x_{F} \leq 1.00$ & 491 & 92 & 19 & 399 \\
\hline $\mathrm{W}$ & $0.00<x_{F} \leq 0.30$ & 1280 & 133 & 10 & 1147 \\
& $0.30<x_{F} \leq 0.41$ & 1885 & 237 & 13 & 1648 \\
& $0.41<x_{F} \leq 0.50$ & 1777 & 219 & 12 & 1558 \\
& $0.50<x_{F} \leq 0.60$ & 1549 & 215 & 14 & 1334 \\
& $0.60<x_{F} \leq 0.67$ & 679 & 103 & 15 & 576 \\
& $0.67<x_{F} \leq 1.00$ & 566 & 134 & 24 & 432 \\
\hline
\end{tabular}

Table 3.16: Summary of background subtraction of Roadset 67 data. 

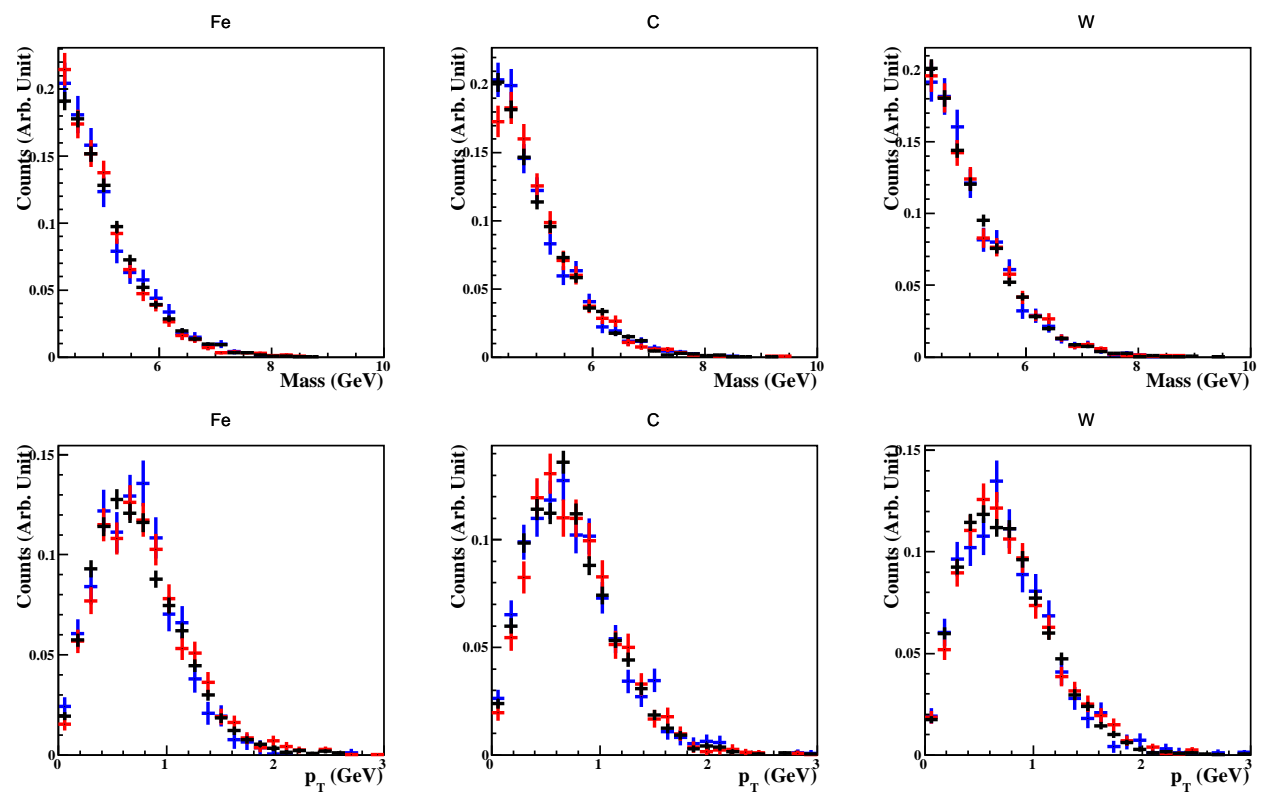

C

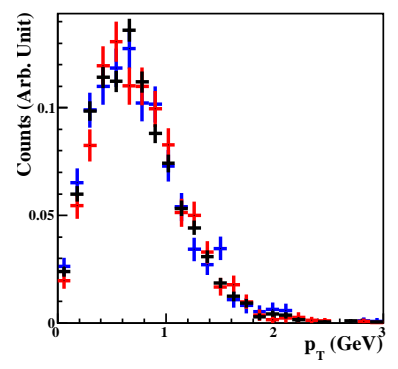

w
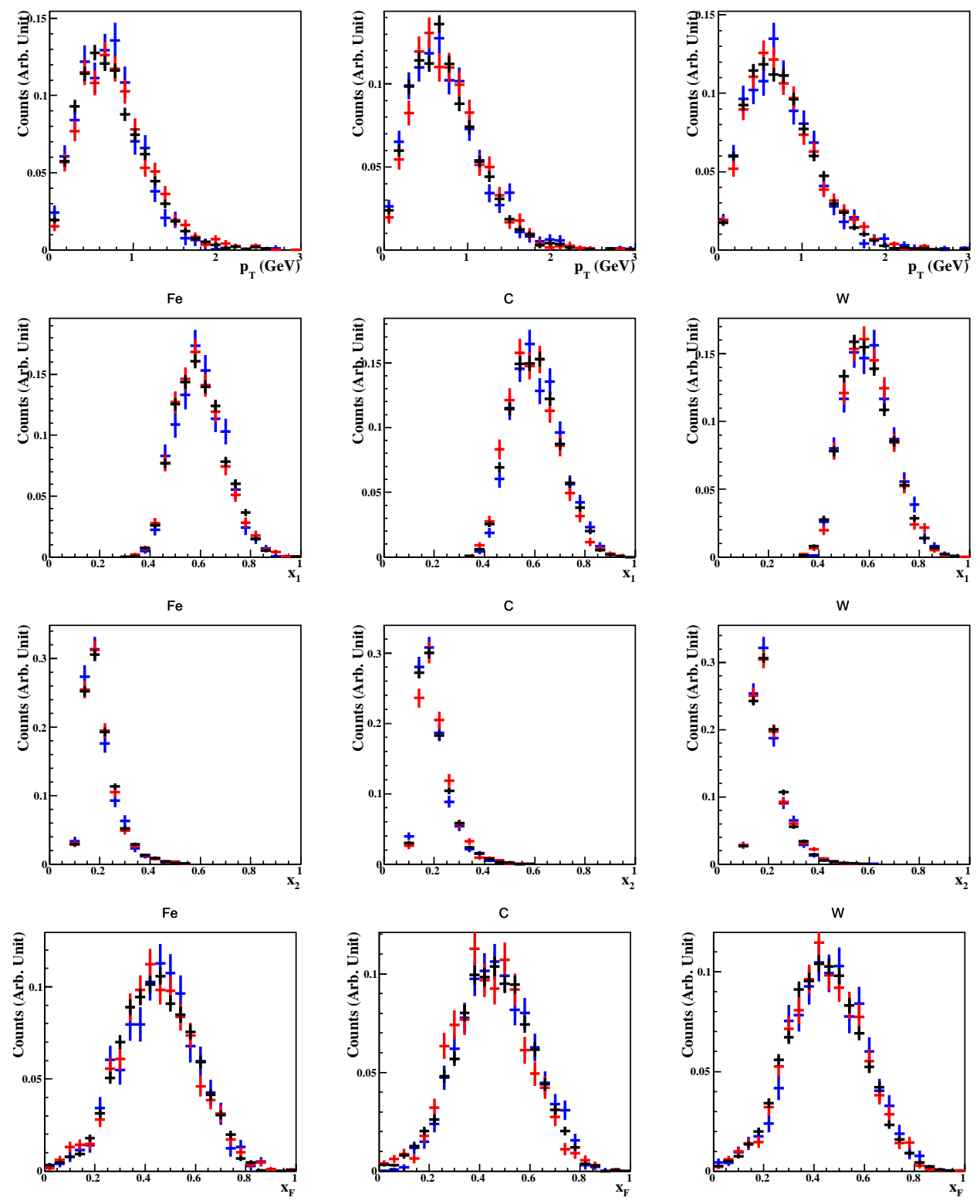

Figure 3.25: Comparison of kinematic variables of mass $>4.2 \mathrm{GeV}$ data after background subtraction from each data sets. The variables are mass, $p_{T}, x_{1}, x_{2}$, and $x_{F}$, from top to bottom. The blue points are from Run-II data, the red points from Roadset 62 data, and the black points from Roadset 67 data. Each distribution of the same target is plotted with the same normalization. 


\begin{tabular}{ccccccc}
\hline Target & $x_{F}$ bin & $\left\langle x_{1}\right\rangle$ & $\left\langle x_{2}\right\rangle$ & $\left\langle x_{F}\right\rangle$ & $\langle$ mass $\rangle$ & $\left\langle p_{T}\right\rangle$ \\
\hline Fe & $0.00<x_{F} \leq 0.30$ & 0.473 & 0.279 & 0.223 & 5.388 & 0.68 \\
& $0.30<x_{F} \leq 0.41$ & 0.535 & 0.221 & 0.359 & 5.080 & 0.74 \\
& $0.41<x_{F} \leq 0.50$ & 0.591 & 0.187 & 0.455 & 4.898 & 0.79 \\
& $0.50<x_{F} \leq 0.60$ & 0.652 & 0.170 & 0.545 & 4.930 & 0.62 \\
& $0.60<x_{F} \leq 0.67$ & 0.710 & 0.149 & 0.630 & 4.793 & 0.70 \\
& $0.67<x_{F} \leq 1.00$ & 0.781 & 0.135 & 0.725 & 4.791 & 0.58 \\
\hline $\mathrm{C}$ & $0.00<x_{F} \leq 0.30$ & 0.477 & 0.271 & 0.237 & 5.352 & 0.66 \\
& $0.30<x_{F} \leq 0.41$ & 0.535 & 0.220 & 0.359 & 5.077 & 0.73 \\
& $0.41<x_{F} \leq 0.50$ & 0.590 & 0.187 & 0.456 & 4.918 & 0.69 \\
& $0.50<x_{F} \leq 0.60$ & 0.657 & 0.174 & 0.548 & 4.979 & 0.72 \\
& $0.60<x_{F} \leq 0.67$ & 0.711 & 0.148 & 0.631 & 4.770 & 0.71 \\
& $0.67<x_{F} \leq 1.00$ & 0.788 & 0.137 & 0.731 & 4.811 & 0.73 \\
\hline $\mathrm{W}$ & $0.00<x_{F} \leq 0.30$ & 0.477 & 0.288 & 0.219 & 5.474 & 0.81 \\
& $0.30<x_{F} \leq 0.41$ & 0.532 & 0.215 & 0.359 & 4.994 & 0.77 \\
& $0.41<x_{F} \leq 0.50$ & 0.593 & 0.189 & 0.457 & 4.931 & 0.75 \\
& $0.50<x_{F} \leq 0.60$ & 0.655 & 0.169 & 0.549 & 4.905 & 0.74 \\
& $0.60<x_{F} \leq 0.67$ & 0.713 & 0.154 & 0.631 & 4.874 & 0.68 \\
& $0.67<x_{F} \leq 1.00$ & 0.787 & 0.147 & 0.726 & 4.977 & 0.76 \\
\hline
\end{tabular}

Table 3.17: Average values of kinematic variables of Run-II data.

\begin{tabular}{ccccccc}
\hline Target & $x_{F}$ bin & $\left\langle x_{1}\right\rangle$ & $\left\langle x_{2}\right\rangle$ & $\left\langle x_{F}\right\rangle$ & $\langle$ mass $\rangle$ & $\left\langle p_{T}\right\rangle$ \\
\hline Fe & $0.00<x_{F} \leq 0.30$ & 0.472 & 0.283 & 0.218 & 5.391 & 0.83 \\
& $0.30<x_{F} \leq 0.41$ & 0.531 & 0.213 & 0.360 & 4.967 & 0.79 \\
& $0.41<x_{F} \leq 0.50$ & 0.590 & 0.188 & 0.455 & 4.907 & 0.76 \\
& $0.50<x_{F} \leq 0.60$ & 0.654 & 0.168 & 0.548 & 4.873 & 0.76 \\
& $0.60<x_{F} \leq 0.67$ & 0.712 & 0.149 & 0.633 & 4.810 & 0.65 \\
& $0.67<x_{F} \leq 1.00$ & 0.791 & 0.145 & 0.732 & 4.933 & 0.79 \\
\hline $\mathrm{C}$ & $0.00<x_{F} \leq 0.30$ & 0.473 & 0.278 & 0.225 & 5.357 & 0.79 \\
& $0.30<x_{F} \leq 0.41$ & 0.537 & 0.220 & 0.361 & 5.081 & 0.76 \\
& $0.41<x_{F} \leq 0.50$ & 0.593 & 0.189 & 0.457 & 4.936 & 0.74 \\
& $0.50<x_{F} \leq 0.60$ & 0.652 & 0.172 & 0.543 & 4.933 & 0.76 \\
& $0.60<x_{F} \leq 0.67$ & 0.715 & 0.156 & 0.632 & 4.917 & 0.67 \\
& $0.67<x_{F} \leq 1.00$ & 0.788 & 0.142 & 0.729 & 4.895 & 0.72 \\
\hline $\mathrm{W}$ & $0.00<x_{F} \leq 0.30$ & 0.481 & 0.290 & 0.222 & 5.514 & 0.82 \\
& $0.30<x_{F} \leq 0.41$ & 0.535 & 0.220 & 0.359 & 5.075 & 0.77 \\
& $0.41<x_{F} \leq 0.50$ & 0.590 & 0.190 & 0.453 & 4.942 & 0.76 \\
& $0.50<x_{F} \leq 0.60$ & 0.654 & 0.167 & 0.549 & 4.857 & 0.75 \\
& $0.60<x_{F} \leq 0.67$ & 0.716 & 0.156 & 0.633 & 4.913 & 0.70 \\
& $0.67<x_{F} \leq 1.00$ & 0.788 & 0.141 & 0.730 & 4.890 & 0.71 \\
\hline
\end{tabular}

Table 3.18: Average values of kinematic variables of Roadset 62 data. 


\begin{tabular}{ccccccc}
\hline Target & $x_{F}$ bin & $\left\langle x_{1}\right\rangle$ & $\left\langle x_{2}\right\rangle$ & $\left\langle x_{F}\right\rangle$ & $\langle$ mass $\rangle$ & $\left\langle p_{T}\right\rangle$ \\
\hline Fe & $0.00<x_{F} \leq 0.30$ & 0.474 & 0.281 & 0.223 & 5.395 & 0.78 \\
& $0.30<x_{F} \leq 0.41$ & 0.532 & 0.218 & 0.358 & 5.038 & 0.73 \\
& $0.41<x_{F} \leq 0.50$ & 0.593 & 0.191 & 0.455 & 4.967 & 0.76 \\
& $0.50<x_{F} \leq 0.60$ & 0.652 & 0.168 & 0.547 & 4.870 & 0.72 \\
& $0.60<x_{F} \leq 0.67$ & 0.717 & 0.158 & 0.633 & 4.940 & 0.72 \\
& $0.67<x_{F} \leq 1.00$ & 0.785 & 0.146 & 0.723 & 4.959 & 0.71 \\
\hline $\mathrm{C}$ & $0.00<x_{F} \leq 0.30$ & 0.474 & 0.283 & 0.220 & 5.416 & 0.77 \\
& $0.30<x_{F} \leq 0.41$ & 0.536 & 0.219 & 0.361 & 5.060 & 0.74 \\
& $0.41<x_{F} \leq 0.50$ & 0.591 & 0.189 & 0.456 & 4.932 & 0.73 \\
& $0.50<x_{F} \leq 0.60$ & 0.653 & 0.169 & 0.547 & 4.896 & 0.72 \\
& $0.60<x_{F} \leq 0.67$ & 0.715 & 0.155 & 0.632 & 4.894 & 0.72 \\
& $0.67<x_{F} \leq 1.00$ & 0.789 & 0.143 & 0.731 & 4.919 & 0.68 \\
\hline $\mathrm{W}$ & $0.00<x_{F} \leq 0.30$ & 0.476 & 0.282 & 0.224 & 5.415 & 0.81 \\
& $0.30<x_{F} \leq 0.41$ & 0.530 & 0.214 & 0.358 & 4.975 & 0.76 \\
& $0.41<x_{F} \leq 0.50$ & 0.591 & 0.189 & 0.454 & 4.938 & 0.73 \\
& $0.50<x_{F} \leq 0.60$ & 0.655 & 0.172 & 0.547 & 4.930 & 0.75 \\
& $0.60<x_{F} \leq 0.67$ & 0.716 & 0.154 & 0.633 & 4.876 & 0.74 \\
& $0.67<x_{F} \leq 1.00$ & 0.792 & 0.145 & 0.733 & 4.957 & 0.74 \\
\hline
\end{tabular}

Table 3.19: Average values of kinematic variables of Roadset 67 data.

\subsubsection{Normalized Yield Ratios after Background Subtraction}

At this stage with the background events filtered out from data, the normalized Drell-Yan yield ratio between heavy targets and carbon can be evaluated as neglecting the correction terms, $F_{A / C}^{R D}$ and $F_{A / C}^{A c p}$, in Eq. 3.2

$$
R_{Y i e l d}=N_{A / C} \cdot \frac{Y_{A}^{D Y}}{Y_{C}^{D Y}}
$$

Three different ratios of iron over carbon $(\mathrm{Fe} / \mathrm{C})$ and tungsten over carbon $(\mathrm{W} / \mathrm{C})$ are made for each data set: the one of events with mass $>4.2 \mathrm{GeV}$, mass $>5 \mathrm{GeV}$, and the one using the normalization of the Monte Carlo distribution acquired in mass spectrum fitting with background counts fixed by the $F_{b k g}$ method. The plots of mass fitting is given in Appendix B. The comparison of the yield ratios is demonstrated in Fig. 3.26, In each data set, the consistency between different ratios serves as another important validity check of the background treatment since the ratio of clean Drell-Yan events is not expected to show noticeable mass dependence in this limited mass 

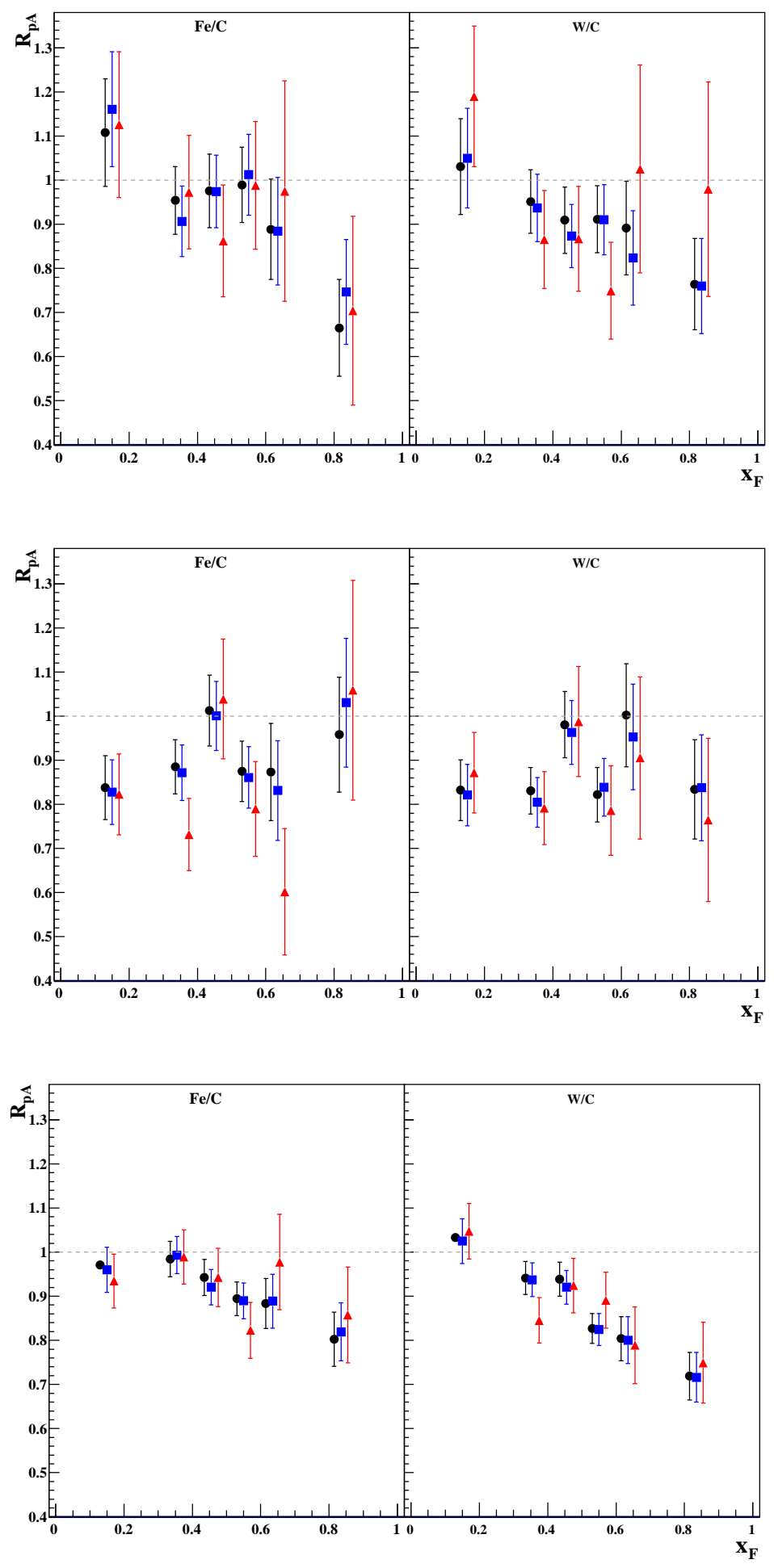

Figure 3.26: Comparison of normalized yield ratios with only statistical errors. The plots correspond to Run-II, Roadset 62 , and Roadset 67 restuls, from top to bottom. The black circles are made by events extracted from mass fitting, the blue squares by mass $>4.2 \mathrm{GeV}$ events, and red triangles by mass $>5 \mathrm{GeV}$ events. 
range. Although the corretion factors are not yet applied, the rate-dependence correction, which depends mostly on the tracking and beam quality, and the acceptance correction are both not expected to significantly depend on mass. The reasons for this statement are going to be discussed in detail in the upcoming sections. For the ratios among different data set, a general agreement between the ratios in each data set can be seen. It is noticed that the overall trend of the Roadset62 result is somewhat different with the other two. Bearing with the large statistical error and the fact that the rate-dependence correction, which is depends on the beam quality can be data-set dependent, is not yet applied, this discrepancy should be acceptable. In addition, although the Drell-Yan yield extracted via fitting has the highest statistics by including events of mass $<4.2$ $\mathrm{GeV}$, it's not going to be used as the input for the cross-section ratio evaluation. This is due to the fact that the shape of Monte Carlo Drell-Yan events, and thus the fitting result, would depend on the PDF set used. To prevent the possible bias brought by a specific PDF choice, and to exclude any influence in fitting brought by the large $J / \psi$ backgrounds, the Drell-Yan yields acquired by simply counting the background-removed events with mass $>4.2 \mathrm{GeV}$ are used for calculating the $R_{p A}$

\subsection{Rate Dependence}

In SeaQuest, rate dependence is used to indicate issues related to the dependencies on the beam intensity. It has been mentioned in Section 2.1 that the intensity of the proton beam delivered to SeaQuest can have severe fluctuations over a spill. As we know, the hit rate on the detector elements is a factor affecting the performance of the tracking for valid dimuon events of both the

hardware and the software level. Since the detector occupancy is expected to be proportional to the beam intensity, the effect correlates with the strongly varying intensity must be carefully studied.

\subsubsection{Reconstruction Efficiency}

The rate dependence on the software level relates to the effectiveness of dimuon reconstruction. In an event with higher occupancy, it is more difficult for the tracker to correctly identify the 
signals generated by the true dimuon from numerous background hits, and the reconstruction of the dimuon is more likely to fail. Due to the fact that the dimuon reconstruction is mostly related to the hits in the chambers, the quantity denoted as the "chamber intensity" is used to better describe the rate dependence of the reconstruction efficiency.

Since the drift time of the signals in the drift chambers can be as long as $260 \mathrm{~ns}$, the occupancy of the chamber can actually be affected by the ions generated by up to 13 buckets before and after the $\mathrm{RF}$ bucket that produces the triggered event of interest. The chamber intensity is defined as the weighted sum of these \pm 13 neighboring buckets. The weight is correlated to the probability for hits produced by tracks generated in a neighboring bucket to be included in the chamber intime window, and can be estimated using the R-T (drift distance versus drift time) curves of the chambers. The chamber intensity therefore accounts for the possible hits from neighboring buckets and would better represent the chamber-hit producing environment that the sense wires in the chambers are facing. In contrast to chamber intensity, another term denoted as the "trigger intensity" simply indicates the number of protons in the triggering bucket.

The rate dependence in SeaQuest was first observed and investigated by Bryan Kerns, a collaborator from the University of Illinois [115]. In his study of the dimuon yield per tigger proton as a function of chamber intensity, a histogram of dimuon yield as a function of chamber intensity was made first. Another histogram of the number of protons from the triggering buckets versus chamber intensity was then produced. The dimuon yield per trigger proton as a function of chamber intensity can then derived as a quotient of these two histograms. Shown in Fig. 3.27 is this trigger-proton normalized dimuon yield as a function of chamber intensity obtained for the liquid deuterium target in Roadset 57. Since the dimuon yield is expected to be proportional to the number of proton-target interactions, and therefore proportional to the number of trigger protons, the dimuon yield per trigger proton versus chamber intensity should be a flat distribution if there were no rate-dependent effect. The suppression in the dimuon yield with higher chamber intensity indicates the tendency that the dimuons generated are more likely to be lost in the higher intensity case and a correction for the rate dependence is necessary. A follow-up study investigated the 


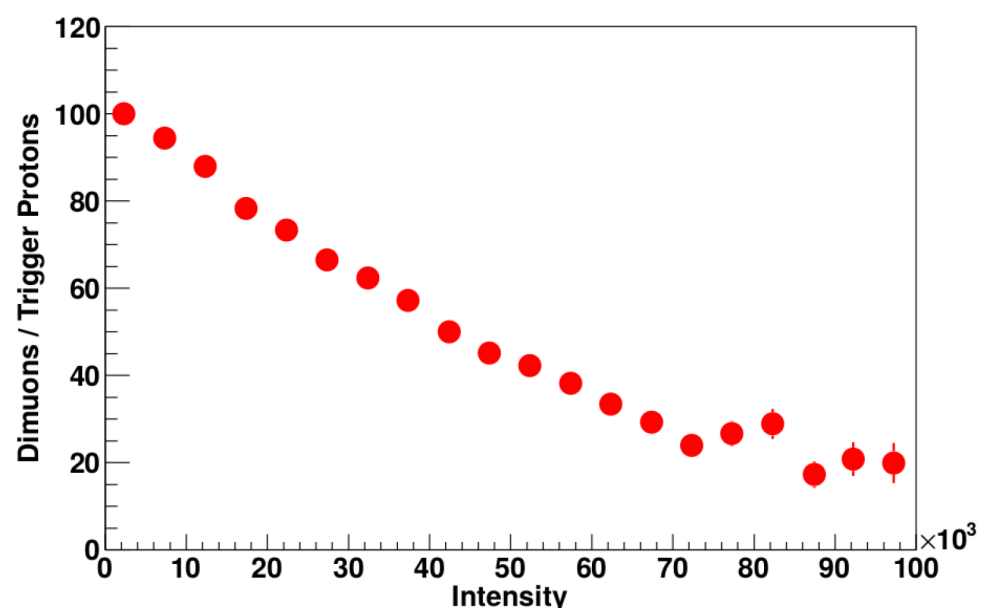

Figure 3.27: Dimuon yield per trigger proton versus chamber intensity of Roadset 57, rescaled so the highest data point is at 100 [115].

hardware-level inefficiency at high intensity could be small, since the high intensity buckets are still not enough to saturate the drift chambers. However, it is difficult to isolate the hardware inefficiency with the necessary involvement of tracking in the analysis. On the other hand, to study the rate dependence of the reconstruction efficiency of the tracking program, the Monte Carlo simulation and the NIM-3 random trigger data are both employed to provide a realistic description of the experimental outcome.

When a dimuon event is generated in the experiment, what the detectors receive are hits from the true dimuons together with hits from the background tracks. To mimic the detector hits, the true dimuon hits are represented by the hits of Monte Carlo Drell-Yan dimuons and the hit information from the NIM-3 data is used as the background. Although the NIM-3 data could include hits from genuine dimuon tracks, the Drell-Yan events are really rare to be recorded with the NIM-3 trigger. It is safe to neglect the possible hit contribution from these valid dimuons. To confirm that the NIM-3 trigger is truly random and not biased, different distributions like the TDC timing, fired detector element, and multiplicity of the NIM-3 data are checked first. To ensure consistency between the NIM-3 data and the FPGA-1 background, the NIM-3 data used should pass the spill-level selection criteria mentioned in Section 3.3.0.1 and then be sorted by the 
corresponding target and by the possibly satisfied FPGA-based trigger. The detector hits from Monte Carlo and from NIM-3 data are then merged together to produce the data with realistic hits.

In order to isolate the inefficiency on reconstruction caused by the detector occupancy, two different kinds of data samples are made:

\section{- Clean data}

The detector hits are the Monte Carlo true hits of Drell-Yan events only.

\section{- Messy data}

The detector hits are the ones with Monte Carlo and NIM-3 data combined.

After merging the detector hits, possible trigger patterns are re-evaluated. The pre-tracking hit reduction and trigger-road masking are thus preformed, and then the tracking and dimuon reconstruction are carried out just like for the real data. As to the beam intensity, both of these two data samples share the same QIE and spill information from the NIM-3 data.

With the clean data sample, the reconstruction inefficiency which is not related to the intensity dependent background hits can be extracted and factored out from the reconstruction inefficiency of the messy data. Therefore, the occupancy dependent reconstruction efficiency, $\epsilon_{R}$, can be acquired as:

$$
\epsilon_{R}=\frac{\epsilon_{\text {messy }}}{\epsilon_{\text {clean }}}
$$

where $\epsilon_{m e s s y}$ and $\epsilon_{\text {clean }}$ represent the binomial efficiency of successfully reconstructed dimuons from the messy and the clean data sample, respectively. In practice, $\epsilon_{R}$ can be obtained directly by taking the ratio of the reconstructed dimuon number from these two data samples, with associated Monte Carlo weighting $w_{i}$ applied on each event. Since $\epsilon_{R}$ can be interpreted as the possibility of getting a positive outcome, which is the successful dimuon reconstruction with the messy data, from a number of trials that is defined as dimuons successfully reconstructed with the clean data, the binomial uncertainty is assigned to $\epsilon_{R}$. By evaluating $\epsilon_{R}$ in different chamber intensity ranges, the 


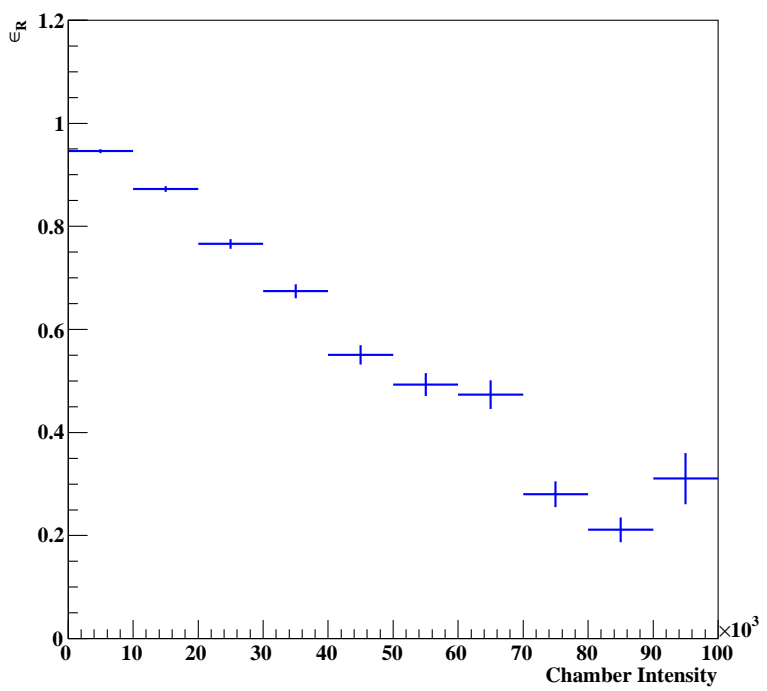

Figure 3.28: Rate dependence of reconstruction efficiency $\epsilon_{R}$ with iron target.

behavior of the rate dependence of $\epsilon_{R}$ can be observed, shown in Fig. 3.28 which used iron target as an example.

\subsubsection{Kinematic Dependence}

What makes the rate dependence more complicated is its possible kinematic dependence. The kinematic dependence of the reconstruction efficiency is related to the fact that the dimuon tracks could tend to populate specific detector regions based on the kinematics of the dimuon. Since we have seen that the detector occupancy can affect the tracking efficiency and the spatial distribution of the hit rate is nonuniform and rises towards the detector edges in general, a kinematic dependence of $\epsilon_{R}$ can arise.

To extract the $x_{F}$ convoluted rate dependence of $\epsilon_{R}$, the distribution of $\epsilon_{R}$ versus chamber intensity is made in each $x_{F}$ binning range, as shown in Fig. 3.29, 3.30, and 3.31 for different targets. The $\epsilon_{R}$ distributions are fitted by a linear function of chamber intensity $I^{C}$

$$
\epsilon_{R}=p_{0}+p_{1} \cdot I^{C}
$$


However, as can be seen in the figures, as $I^{C}$ becomes higher than 60,000 , the linearity of $\epsilon_{R}$ versus $I^{C}$ becomes worse and the $\epsilon_{R}$ values are more scattered in general. This indicates that the tracker might be reaching its limit and is not working reliably in the very high intensity case. Since only a small fraction of the data has $I^{C}$ greater than 60,000 (about $2 \%$ in Roadset 67 for example), we decided to evaluate the $\epsilon_{R}$ rate dependence in the following way:

- Perform the linear fitting of $\epsilon_{R}$ in the $I^{C}$ range from 0 to 60,000 , and acquire the fitted slope and its error $p_{1}^{\text {short }} \pm \delta p_{1}^{\text {short }}$. Use the $p_{1}^{\text {short }}$ as the primary result of $p_{1}$.

- Also do the linear fitting in a wider $I^{C}$ range, from 0 to 80,000. Compare the result, $p_{1}^{\text {wide }} \pm \delta p_{1}^{\text {wide }}$, with $p_{1}^{\text {short }} \pm \delta p_{1}^{\text {short }}$ and take the largest difference with $p_{1}^{\text {short }}$ as the primary error $\delta p_{1}$.

The reason for not eliminating data with $I^{C}>60,000$ is due to the fact that $I^{C}$ is a weighted sum of neighboring buckets, while the number of protons used in the normalization of cross section includes triggering bucket only. Simply removing the events based on $I^{C}$ raises concerns about the overall normalization and we decided not to do so.

The fitting parameters $p_{0}$ and $p_{1}$ obtained in the $I^{C}$ range of 0 to 60,000 are listed in Tab. 3.20, 3.21, and 3.22 for each data set. As can be seen in the fitting results, the parameter $p_{0}$ is close to, or agrees with 1 within error in general, which matches our expectation that the occupancy dependent efficiency should be 1 as the chamber intensity approaches zero and the tracking program is seeing diminishing background hits. What is more important is the clear correlation between rate dependence of $\epsilon_{R}$ and $x_{F}$ for all three targets. As the $x_{F}$ rises, the rate dependence is mitigated, which is to say that the suppression of $\epsilon_{R}$ at higher chamber intensity is weaker. The reason for this $x_{F}$ dependence can be explained by the connection between $x_{F}$ and the detector hit distribution of the dimuon tracks. 

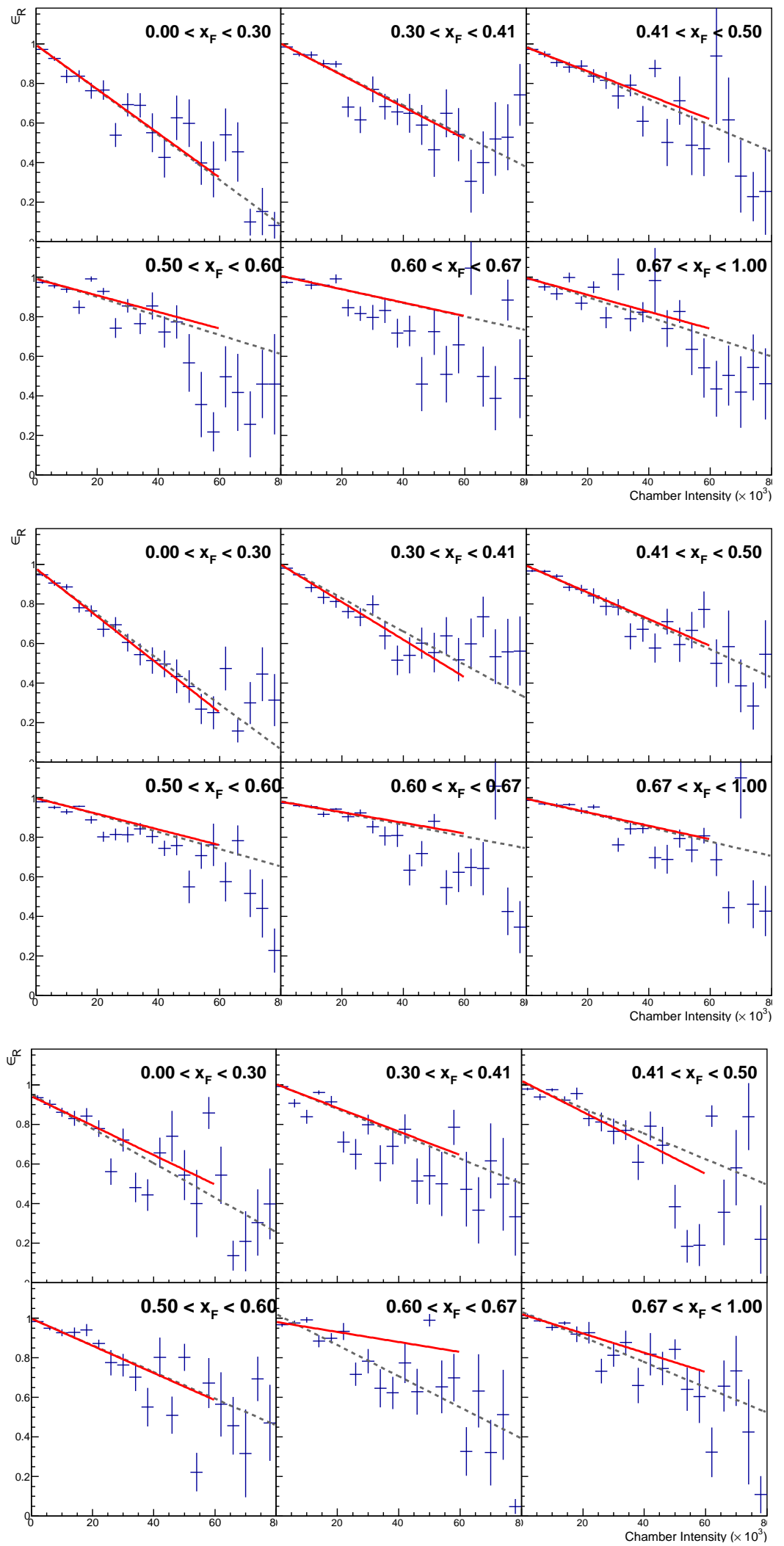

Figure 3.29: $\epsilon_{R}$ fitting of Run-II in $I^{C}$ range of 0 to $60 \mathrm{~K}(80 \mathrm{~K})$, shown as the solid (dashed) lines. From top to bottom, the groups of six plots correspond to iron, carbon, and tungsten results. 


\begin{tabular}{lcccc} 
Iron & \multicolumn{5}{l}{} \\
\hline$x_{F}$ bin & $p_{0}$ & $\delta p_{0}$ & $p_{1}$ & $\delta p_{1}$ \\
\hline $0.00<x_{F} \leq 0.30$ & 0.9932 & 0.0029 & $-1.1157 \mathrm{E}-05$ & $6.6997 \mathrm{E}-07$ \\
$0.30<x_{F} \leq 0.41$ & 1.0010 & 0.0036 & $-8.0169 \mathrm{E}-06$ & $7.4169 \mathrm{E}-07$ \\
$0.41<x_{F} \leq 0.50$ & 0.9822 & 0.0070 & $-6.0747 \mathrm{E}-06$ & $1.1086 \mathrm{E}-06$ \\
$0.50<x_{F} \leq 0.60$ & 0.9913 & 0.0069 & $-4.1718 \mathrm{E}-06$ & $1.1121 \mathrm{E}-06$ \\
$0.60<x_{F} \leq 0.67$ & 1.0068 & 0.0048 & $-3.3774 \mathrm{E}-06$ & $3.9664 \mathrm{E}-07$ \\
$0.67<x_{F} \leq 1.00$ & 0.9966 & 0.0052 & $-4.2761 \mathrm{E}-06$ & $1.2345 \mathrm{E}-06$ \\
\hline
\end{tabular}

\begin{tabular}{lcccc} 
Carbon & \multicolumn{5}{l}{} \\
\hline$x_{F}$ bin & $p_{0}$ & $\delta p_{0}$ & $p_{1}$ & $\delta p_{1}$ \\
\hline $0.00<x_{F} \leq 0.30$ & 0.9778 & 0.0074 & $-1.2102 \mathrm{E}-05$ & $1.2095 \mathrm{E}-06$ \\
$0.30<x_{F} \leq 0.41$ & 0.9985 & 0.0025 & $-9.5014 \mathrm{E}-06$ & $1.5722 \mathrm{E}-06$ \\
$0.41<x_{F} \leq 0.50$ & 0.9931 & 0.0055 & $-6.7662 \mathrm{E}-06$ & $7.9810 \mathrm{E}-07$ \\
$0.50<x_{F} \leq 0.60$ & 0.9973 & 0.0041 & $-3.9431 \mathrm{E}-06$ & $6.9854 \mathrm{E}-07$ \\
$0.60<x_{F} \leq 0.67$ & 0.9804 & 0.0037 & $-2.6775 \mathrm{E}-06$ & $5.7608 \mathrm{E}-07$ \\
$0.67<x_{F} \leq 1.00$ & 0.9946 & 0.0014 & $-3.4102 \mathrm{E}-06$ & $3.9758 \mathrm{E}-07$ \\
\hline
\end{tabular}

\begin{tabular}{lcccc} 
Tungsten & \multicolumn{5}{l}{} \\
\hline$x_{F}$ bin & $p_{0}$ & $\delta p_{0}$ & $p_{1}$ & $\delta p_{1}$ \\
\hline $0.00<x_{F} \leq 0.30$ & 0.9417 & 0.0117 & $-7.4318 \mathrm{E}-06$ & $1.8563 \mathrm{E}-06$ \\
$0.30<x_{F} \leq 0.41$ & 1.0023 & 0.0035 & $-5.9708 \mathrm{E}-06$ & $8.3076 \mathrm{E}-07$ \\
$0.41<x_{F} \leq 0.50$ & 1.0196 & 0.0069 & $-7.8336 \mathrm{E}-06$ & $1.8888 \mathrm{E}-06$ \\
$0.50<x_{F} \leq 0.60$ & 0.9980 & 0.0049 & $-6.8702 \mathrm{E}-06$ & $6.3144 \mathrm{E}-07$ \\
$0.60<x_{F} \leq 0.67$ & 0.9812 & 0.0079 & $-2.5338 \mathrm{E}-06$ & $5.7108 \mathrm{E}-06$ \\
$0.67<x_{F} \leq 1.00$ & 1.0208 & 0.0068 & $-4.8735 \mathrm{E}-06$ & $1.9985 \mathrm{E}-06$ \\
\hline
\end{tabular}

Table 3.20: Parameters of rate-dependent $\epsilon_{R}$ fitting results in the chamber-intensity range of 0 to 60,000 of Run-II . 

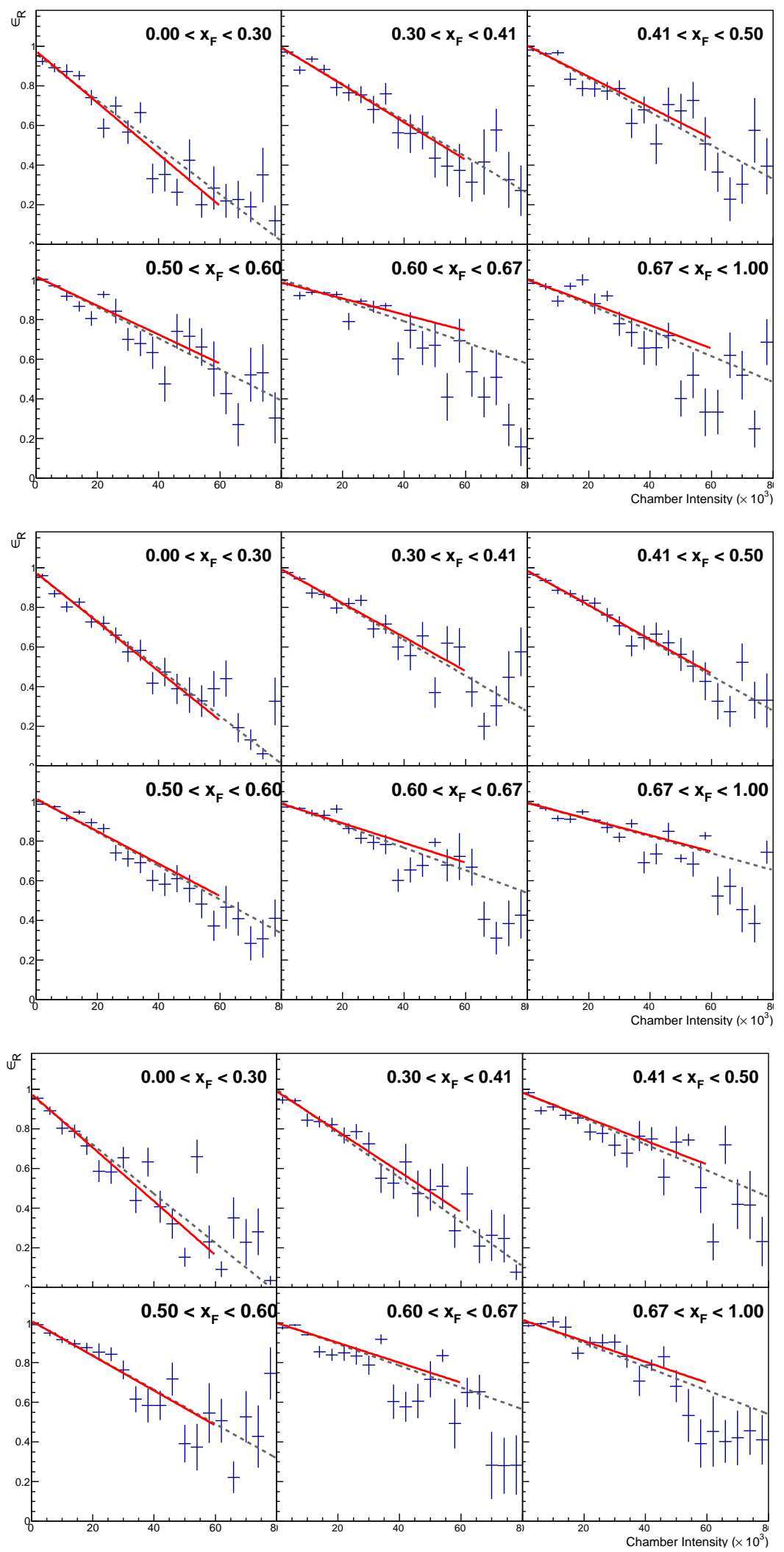

Figure 3.30: $\epsilon_{R}$ fitting of Roadset 62 in $I^{C}$ range of 0 to $60 \mathrm{~K}(80 \mathrm{~K})$, shown as the solid (dashed) lines. From top to bottom, the groups of six plots correspond to iron, carbon, and tungsten results. 


\begin{tabular}{lcccc} 
Iron & \multicolumn{5}{l}{} \\
\hline$x_{F}$ bin & $p_{0}$ & $\delta p_{0}$ & $p_{1}$ & $\delta p_{1}$ \\
\hline $0.00<x_{F} \leq 0.30$ & 0.9738 & 0.0139 & $-1.2992 \mathrm{E}-05$ & $1.6979 \mathrm{E}-06$ \\
$0.30<x_{F} \leq 0.41$ & 0.9929 & 0.0092 & $-9.4467 \mathrm{E}-06$ & $8.6496 \mathrm{E}-07$ \\
$0.41<x_{F} \leq 0.50$ & 1.0014 & 0.0059 & $-7.7786 \mathrm{E}-06$ & $1.1549 \mathrm{E}-06$ \\
$0.50<x_{F} \leq 0.60$ & 1.0160 & 0.0057 & $-7.2886 \mathrm{E}-06$ & $1.0144 \mathrm{E}-06$ \\
$0.60<x_{F} \leq 0.67$ & 0.9877 & 0.0076 & $-4.0575 \mathrm{E}-06$ & $1.6475 \mathrm{E}-06$ \\
$0.67<x_{F} \leq 1.00$ & 1.0026 & 0.0065 & $-5.7846 \mathrm{E}-06$ & $1.1857 \mathrm{E}-06$ \\
\hline
\end{tabular}

\begin{tabular}{lcccc} 
Carbon & \multicolumn{5}{l}{} \\
\hline$x_{F}$ bin & $p_{0}$ & $\delta p_{0}$ & $p_{1}$ & $\delta p_{1}$ \\
\hline $0.00<x_{F} \leq 0.30$ & 0.9735 & 0.0090 & $-1.2413 \mathrm{E}-05$ & $7.5275 \mathrm{E}-07$ \\
$0.30<x_{F} \leq 0.41$ & 0.9904 & 0.0074 & $-8.5375 \mathrm{E}-06$ & $8.7795 \mathrm{E}-07$ \\
$0.41<x_{F} \leq 0.50$ & 0.9839 & 0.0073 & $-8.6518 \mathrm{E}-06$ & $6.1734 \mathrm{E}-07$ \\
$0.50<x_{F} \leq 0.60$ & 1.0128 & 0.0045 & $-8.1646 \mathrm{E}-06$ & $6.7662 \mathrm{E}-07$ \\
$0.60<x_{F} \leq 0.67$ & 0.9896 & 0.0048 & $-4.9605 \mathrm{E}-06$ & $1.0459 \mathrm{E}-06$ \\
$0.67<x_{F} \leq 1.00$ & 0.9929 & 0.0036 & $-4.0845 \mathrm{E}-06$ & $3.6165 \mathrm{E}-07$ \\
\hline
\end{tabular}

\begin{tabular}{lcccc} 
Tungsten & \multicolumn{5}{l}{} \\
\hline$x_{F}$ bin & $p_{0}$ & $\delta p_{0}$ & $p_{1}$ & $\delta p_{1}$ \\
\hline $0.00<x_{F} \leq 0.30$ & 0.9752 & 0.0094 & $-1.3527 \mathrm{E}-05$ & $1.4215 \mathrm{E}-06$ \\
$0.30<x_{F} \leq 0.41$ & 0.9889 & 0.0116 & $-1.0152 \mathrm{E}-05$ & $1.4145 \mathrm{E}-06$ \\
$0.41<x_{F} \leq 0.50$ & 0.9813 & 0.0065 & $-6.0281 \mathrm{E}-06$ & $9.6120 \mathrm{E}-07$ \\
$0.50<x_{F} \leq 0.60$ & 1.0101 & 0.0042 & $-8.7761 \mathrm{E}-06$ & $5.8405 \mathrm{E}-07$ \\
$0.60<x_{F} \leq 0.67$ & 1.0004 & 0.0045 & $-5.0096 \mathrm{E}-06$ & $8.4944 \mathrm{E}-07$ \\
$0.67<x_{F} \leq 1.00$ & 1.0147 & 0.0051 & $-5.2538 \mathrm{E}-06$ & $1.1363 \mathrm{E}-06$ \\
\hline
\end{tabular}

Table 3.21: Parameters of rate-dependent $\epsilon_{R}$ fitting results in the chamber-intensity range of 0 to 60,000 of Roadset 62 . 

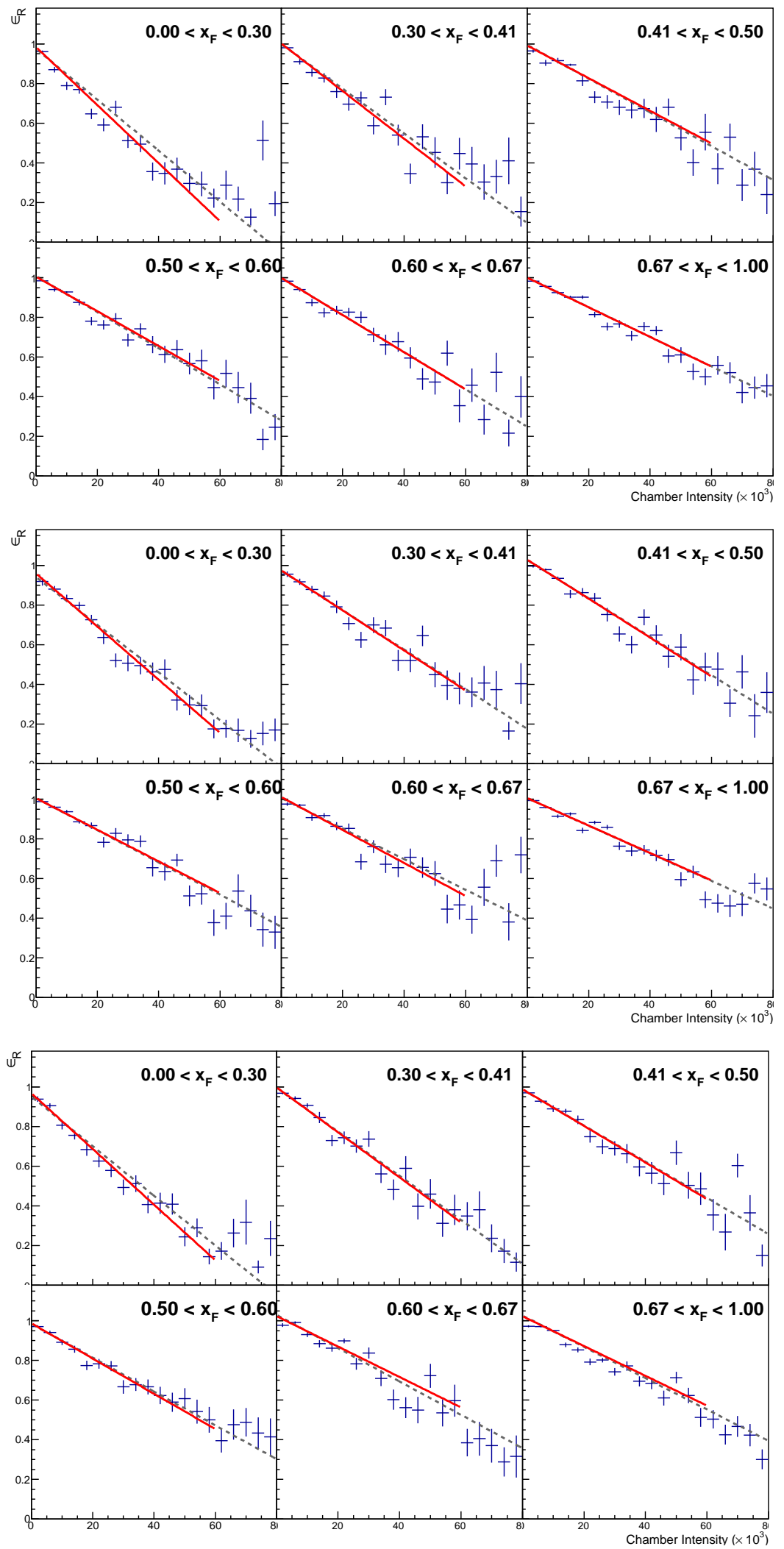

Figure 3.31: $\epsilon_{R}$ fitting of Roadset 67 in $I^{C}$ range of 0 to $60 \mathrm{~K}(80 \mathrm{~K})$, shown as the solid (dashed) lines. From top to bottom, the groups of six plots correspond to iron, carbon, and tungsten results. 


\begin{tabular}{lcccc} 
Iron & \multicolumn{5}{l}{} \\
\hline$x_{F}$ bin & $p_{0}$ & $\delta p_{0}$ & $p_{1}$ & $\delta p_{1}$ \\
\hline $0.00<x_{F} \leq 0.30$ & 0.9830 & 0.0048 & $-1.4620 \mathrm{E}-05$ & $2.0754 \mathrm{E}-06$ \\
$0.30<x_{F} \leq 0.41$ & 1.0021 & 0.0042 & $-1.2024 \mathrm{E}-05$ & $1.0833 \mathrm{E}-06$ \\
$0.41<x_{F} \leq 0.50$ & 0.9916 & 0.0067 & $-8.2395 \mathrm{E}-06$ & $6.4874 \mathrm{E}-07$ \\
$0.50<x_{F} \leq 0.60$ & 1.0057 & 0.0036 & $-8.7678 \mathrm{E}-06$ & $5.9687 \mathrm{E}-07$ \\
$0.60<x_{F} \leq 0.67$ & 0.9995 & 0.0053 & $-9.3772 \mathrm{E}-06$ & $4.2723 \mathrm{E}-07$ \\
$0.67<x_{F} \leq 1.00$ & 1.0009 & 0.0032 & $-7.4793 \mathrm{E}-06$ & $2.3583 \mathrm{E}-07$ \\
\hline
\end{tabular}

\begin{tabular}{lcccc} 
Carbon & \multicolumn{5}{l}{} \\
\hline$x_{F}$ bin & $p_{0}$ & $\delta p_{0}$ & $p_{1}$ & $\delta p_{1}$ \\
\hline $0.00<x_{F} \leq 0.30$ & 0.9581 & 0.0111 & $-1.3379 \mathrm{E}-05$ & $1.7123 \mathrm{E}-06$ \\
$0.30<x_{F} \leq 0.41$ & 0.9747 & 0.0104 & $-1.0106 \mathrm{E}-05$ & $5.5088 \mathrm{E}-07$ \\
$0.41<x_{F} \leq 0.50$ & 1.0284 & 0.0051 & $-9.8216 \mathrm{E}-06$ & $4.8515 \mathrm{E}-07$ \\
$0.50<x_{F} \leq 0.60$ & 1.0051 & 0.0019 & $-7.9586 \mathrm{E}-06$ & $3.9659 \mathrm{E}-07$ \\
$0.60<x_{F} \leq 0.67$ & 1.0114 & 0.0059 & $-8.3193 \mathrm{E}-06$ & $9.6682 \mathrm{E}-07$ \\
$0.67<x_{F} \leq 1.00$ & 1.0060 & 0.0021 & $-6.9227 \mathrm{E}-06$ & $2.1872 \mathrm{E}-07$ \\
\hline
\end{tabular}

\begin{tabular}{lcccc} 
Tungsten & \multicolumn{5}{l}{} \\
\hline$x_{F}$ bin & $p_{0}$ & $\delta p_{0}$ & $p_{1}$ & $\delta p_{1}$ \\
\hline $0.00<x_{F} \leq 0.30$ & 0.9666 & 0.0092 & $-1.4027 \mathrm{E}-05$ & $1.8395 \mathrm{E}-06$ \\
$0.30<x_{F} \leq 0.41$ & 0.9973 & 0.0052 & $-1.1348 \mathrm{E}-05$ & $5.4908 \mathrm{E}-07$ \\
$0.41<x_{F} \leq 0.50$ & 0.9876 & 0.0048 & $-9.2286 \mathrm{E}-06$ & $4.3272 \mathrm{E}-07$ \\
$0.50<x_{F} \leq 0.60$ & 0.9867 & 0.0054 & $-8.8772 \mathrm{E}-06$ & $6.6688 \mathrm{E}-07$ \\
$0.60<x_{F} \leq 0.67$ & 1.0236 & 0.0061 & $-7.6758 \mathrm{E}-06$ & $1.0819 \mathrm{E}-06$ \\
$0.67<x_{F} \leq 1.00$ & 1.0230 & 0.0026 & $-7.5200 \mathrm{E}-06$ & $5.8095 \mathrm{E}-07$ \\
\hline
\end{tabular}

Table 3.22: Parameters of rate-dependent $\epsilon_{R}$ fitting results in the chamber-intensity range of 0 to 60,000 of Roadset 67 . 

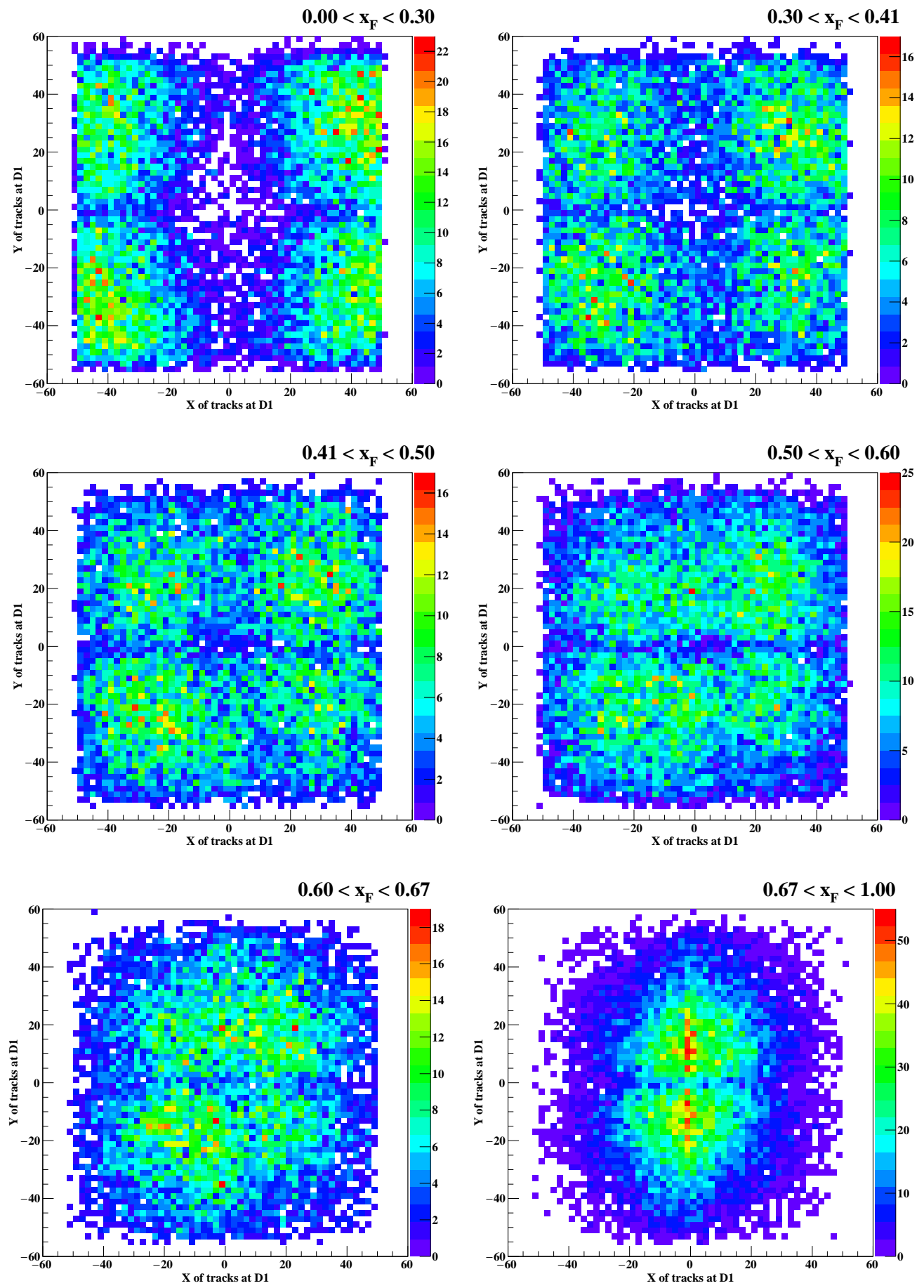

Figure 3.32: D1 hit position of the tracks of the dimuons lost in reconstruction in different $x_{F}$ ranges. Iron target data was used.

Fig. 3.32 shows the station 1 drift chamber hit distribution of the tracks that are not success- 
fully reconstructed. As can be seen, since $x_{F}$ represents the fraction of the longitudinal momentum that can be possibly carried by the dimuon, with higher $x_{F}$ the muon tracks would be boost forward more in the positive $\mathrm{Z}$ direction and therefore be more concentrated in the central part of the detectors. As is illustrated in the hit distribution of D3p in Fig. 2.10 for example, the hit occupancy of the spectrometer is smallest around the center and gradually increases as moving toward the outer edge. Consequently, the effect of rate dependency would be stronger for the events that have their tracks penetrating through the outer part of the detectors, which are the events with smaller $x_{F}$. Additionally, target dependence on the fitting results can be observed. As we know, the interaction lengths of the solid targets are different from each other, so the number of background tracks generated and thus the detector occupancy can be target dependent. These results suggest that this target difference could only have minor effect on rate-dependent inefficiency. Still, each target is treated differently when making the rate-dependence corrections.

\subsubsection{Correction for Reconstruction Efficiency}

To compensate the rate-dependent reconstruction inefficiency, since the observed Drell-Yan yield is the true yield times the efficiency, we can apply a correction factor which is the inverse of the extracted efficiency to the observed Drell-Yan yield to correct it back. As explained previously, this correction factor $C^{c o r}$ would be a function of the chamber intensity $I^{C}$ and $x_{F}$, and can be evaluated as

$$
C^{c o r}\left(I^{C}, x_{F}\right)=\frac{1}{1-p_{1}\left(x_{F}\right) \cdot I^{C}}
$$

where the $p_{1}\left(x_{F}\right)$ is the fitting extracted value in a specific $x_{F}$ bin, as listed in Tab. $3.20,3.21$, and 3.22 . And the uncertainty of $C^{\text {cor }}$ is estimated as

$$
\delta C^{c o r}\left(I^{C}, x_{F}\right)=\frac{I^{C} \cdot \delta p_{1}\left(x_{F}\right)}{\left[1-p_{1}\left(x_{F}\right) \cdot I^{C}\right]^{2}}
$$

in which the fitting error of $p_{1}$ is used as $\delta p_{1}\left(x_{F}\right)$. The resulting $F_{A / C}^{R D}$ uncertainty caused by $\delta C^{c o r}$ will be treated as the systematic error, denoted as $\delta F_{A / C}^{s y s-R D f i t}$.

In Eq. 3.17 given above, it can be noticed that the denominator is a little different from the 
expression of $\epsilon_{R}$ in Eq. 3.16 - the parameter $p_{0}$ is replaced by the constant 1 . We have seen in the discussion before that the $p_{0}$ values obtained from fitting are consistent with one, as they should be. Since the overall distribution of $\epsilon_{R}$ versus chamber intensity could be slightly shifted horizontally due to things like the possible drifting of the QIE pedestal value and cause $p_{0}$ to deviate from 1 , it is more reasonable to set the $p_{0}$ as one in the evaluation of $C^{c o r}$. We can then obtain the rate-dependence correction factor $F_{A / C}^{R D}$ used in Eq. 3.2 by doing the ratio of $C^{c o r}$ between the carbon and other heavier nuclear targets as

$$
F_{A / C}^{R D}=C_{A}^{c o r} / C_{C}^{c o r}
$$

However, there is an additional issue, which is also related to the background, that needs to be considered.

As been discussed already, the Drell-Yan yield in each $x_{F}$ bin is obtained by subtracting the measured raw-event count by the estimated number of background. In order to make the reconstruction efficiency correction bin by bin, as indicated in Eq. 3.17, it is necessary to have the $I^{C}$ values of both the raw and background events so the ones of the Drell-Yan events can be acquired for calculating $C^{c o r}$. However, since there is no obvious method to get the chamber intensity of the subtracted background, it is a non-trivial job to do.

In the case that the $I^{C}$ distribution of the background is similar to that of the Drell-Yan events, we can assume that the $I^{C}$ average of the raw events, without background subtraction, is about the same as the average of the Drell-Yan events. Since the $\epsilon_{R}$ is a linear function of $I^{C}$, it would be fine to use the bin-average $I^{C}$ of the raw events in each $x_{F}$ bin for the evaluation of $C^{c o r}$, and make the correction bin by bin. To check the validity of the statement above, the $I^{C}$ distributions of three different mass ranges, $0<$ mass $<10 \mathrm{GeV}$, mass $>4.2 \mathrm{GeV}$, and mass $<2.5$ $\mathrm{GeV}$, are examined and shown in Fig. 3.33 .

In Fig. 3.33 we can see that although being shifted towards higher $I^{C}$, the $I^{C}$ distribution of $0<$ mass $<10 \mathrm{GeV}$, which is basically the whole data, is quite similar to the one of mass $>4.2$ $\mathrm{GeV}$, in which the Drell-Yan events dominates. The $I^{C}$ of mass $<2.5 \mathrm{GeV}$ data that consists 


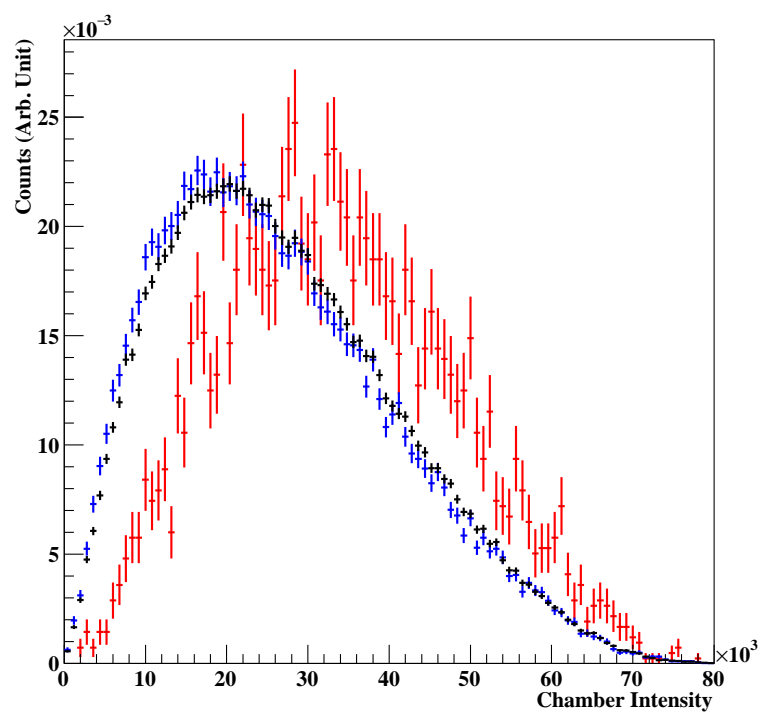

Figure 3.33: Chamber intensity distributions in different mass ranges of Roadset 67 . The black points are mass $>0$ and $<10 \mathrm{GeV}$ data, the blue ones are mass $>4.2 \mathrm{GeV}$ data, and the red ones are mass $<2.5 \mathrm{GeV}$ data. The area of each distribution is normalized to 1 .

of background, however, populates more at higher chamber intensity and is noticeably different from the other two. The discrepancy is reasonable since with higher intensity, we would expect more random tracks to be produced and therefore higher possibility to have the background events generated. Although the comparison is made in different mass range, it highly suggests the possible difference between the $I^{C}$ distribution of Drell-Yan and background events with mass $>4.2 \mathrm{GeV}$ and consequently the necessity of removing the contribution from the background in evaluating the average $I^{C}$.

Under the circumstance that the direct extraction of the $I^{C}$ of mass $>4.2 \mathrm{GeV}$ background could not be done, an alternative approach, which assumes the background $I^{C}$ distribution is not mass dependent, is taken to handle the background subtraction in $I^{C}$. With this assumption, we claim that the $I^{C}$ distribution of mass $>4.2$ background could be represented by the one from mass $<2.5 \mathrm{GeV}$ data. Since the events from the none targets should also be dominated by combinatorial background, we check the assumption that the background $I^{C}$ does not have mass dependence by comparing the $I^{C}$ distribution of difference mass range of the none target. As can be seen in 


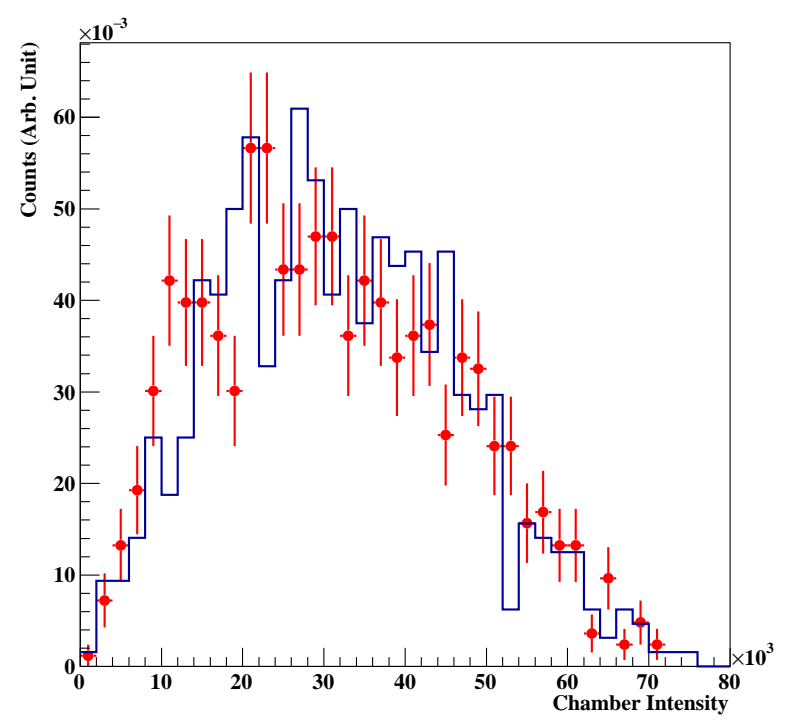

Figure 3.34: Chamber intensity of "none" target for different mass ranges of Roadset 67 plotted with the same normalization. The blue histogram is the intensity of mass $>3.5 \mathrm{GeV}$ and the red points represent the intensity of mass $<3.5 \mathrm{GeV}$.

Fig. 3.34, there is no obvious disagreement between the $I^{C}$ distributions of high mass and low mass, and this can be an indirect verification of the background intensity treatment. The average $I^{C}$ in a specific $x_{F}$ bin can then be derived with the following procedures:

(1) Make the $I^{C}$ distribution of mass $<2.5 \mathrm{GeV}$ data, and scale this distribution so its normalization is identical to the estimated number of background events of the $x_{F}$ bin with masss $>4.2 \mathrm{GeV}$. This distribution is denoted as $I^{C-B G}$

(2) Make the $I^{C}$ distribution of mass $>4.2 \mathrm{GeV}$ data in the $x_{F}$ bin. Subtract this distribution by $I^{C-B G}$ and obtain $I^{C-D Y}$.

(3) Evaluate the average of $I^{C-D Y}$, which is then used as the average $I^{C}$ of this $x_{F}$ bin.

Obviously, the average $I^{C}$ obtained depends on the histogram binning of the distribution and is expected to be more accurate with finer binning. It is observed that with the number of bins greater than 200, the change in $C^{c o r}$ acquired is just less than $1 \%$. The $I^{C}$ average is thus evaluated with histogram of 200 bins. 
Indeed, this approach may not be totally reliable, but should be the most reasonable way at this point. To estimate the uncertainty brought by this treatment, we also evaluated the correction factor in the extreme case, in which no background subtraction is done in calculating the average $I^{C}$. The $C^{c o r}$ acquired without background treatment is compared to the one obtained in the approach mentioned above, and the difference is taken as the systematic error. This error, as it propagates to the calculation of $F_{A / C}^{R D}$, is denoted as $\delta F_{A / C}^{s y s-R D I}$. For each Roadset, the $F_{A / C}^{R D}$ numbers are summarized in Tab. 3.24, 3.25, and 3.26, together with the pictorial illustrations in Fig. 3.35, 3.36, and 3.37.

\begin{tabular}{lcccccc} 
Run-II & \multicolumn{7}{l}{} \\
\hline$x_{F}$ bin & $\left\langle I_{F e}^{C}\right\rangle$ & $C_{F e}^{c o r}$ & $\left\langle I_{C}^{C}\right\rangle$ & $C_{C}^{\text {cor }}$ & $\left\langle I_{W}^{C}\right\rangle$ & $C_{W}^{\text {cor }}$ \\
\hline $0.00<x_{F} \leq 0.30$ & 21172.7 & 1.309 & 19708.0 & 1.313 & 21436.0 & 1.189 \\
$0.30<x_{F} \leq 0.41$ & 24316.8 & 1.242 & 23138.8 & 1.282 & 24980.9 & 1.175 \\
$0.41<x_{F} \leq 0.50$ & 23119.2 & 1.163 & 23737.7 & 1.191 & 23730.0 & 1.228 \\
$0.50<x_{F} \leq 0.60$ & 22526.7 & 1.104 & 23543.8 & 1.102 & 22554.0 & 1.183 \\
$0.60<x_{F} \leq 0.67$ & 24436.1 & 1.090 & 25164.3 & 1.072 & 23735.4 & 1.064 \\
$0.67<x_{F} \leq 1.00$ & 19686.2 & 1.092 & 24261.9 & 1.090 & 25128.0 & 1.140 \\
\hline
\end{tabular}

\begin{tabular}{|c|c|c|c|c|c|c|}
\hline \multicolumn{7}{|l|}{ Roadset 62} \\
\hline$x_{F}$ bin & $\left\langle I_{F e}^{C}\right\rangle$ & $C_{F e}^{c o r}$ & $\left\langle I_{C}^{C}\right\rangle$ & $C_{C}^{c o r}$ & $\left\langle I_{W}^{C}\right\rangle$ & $C_{W}^{c o r}$ \\
\hline $0.00<x_{F} \leq 0.30$ & 23121.7 & 1.429 & 22631.8 & 1.391 & 21832.7 & 1.419 \\
\hline $0.30<x_{F} \leq 0.41$ & 24788.4 & 1.306 & 24567.1 & 1.265 & 22981.1 & 1.304 \\
\hline $0.41<x_{F} \leq 0.50$ & 23719.0 & 1.226 & 24764.2 & 1.273 & 25115.8 & 1.178 \\
\hline $0.50<x_{F} \leq 0.60$ & 23204.9 & 1.204 & 24599.3 & 1.251 & 25369.6 & 1.286 \\
\hline $0.60<x_{F} \leq 0.67$ & 23202.8 & 1.104 & 25133.0 & 1.142 & 26528.2 & 1.153 \\
\hline $0.67<x_{F} \leq 1.00$ & 25745.1 & 1.175 & 28069.2 & 1.129 & 25509.0 & 1.155 \\
\hline
\end{tabular}

\begin{tabular}{|c|c|c|c|c|c|c|}
\hline \multicolumn{7}{|l|}{ Roadset 67} \\
\hline$x_{F}$ bin & $\left\langle I_{F e}^{C}\right\rangle$ & $C_{F e}^{c o r}$ & $\left\langle I_{C}^{C}\right\rangle$ & $C_{C}^{c o r}$ & $\left\langle I_{W}^{C}\right\rangle$ & $C_{W}^{c o r}$ \\
\hline $0.00<x_{F} \leq 0.30$ & 22893.9 & 1.503 & 23442.3 & 1.457 & 24341.9 & 1.518 \\
\hline $0.30<x_{F} \leq 0.41$ & 25003.2 & 1.430 & 25368.7 & 1.345 & 25086.2 & 1.398 \\
\hline $0.41<x_{F} \leq 0.50$ & 25505.5 & 1.266 & 26273.7 & 1.348 & 25445.6 & 1.307 \\
\hline $0.50<x_{F} \leq 0.60$ & 25394.2 & 1.286 & 25689.3 & 1.257 & 25974.1 & 1.300 \\
\hline $0.60<x_{F} \leq 0.67$ & 25900.1 & 1.321 & 26361.3 & 1.281 & 26394.7 & 1.254 \\
\hline $0.67<x_{F} \leq 1.00$ & 26230.4 & 1.244 & 26279.4 & 1.222 & 26597.3 & 1.250 \\
\hline
\end{tabular}

Table 3.23: Average chamber intensity and $C^{c o r}$. 
Run-II

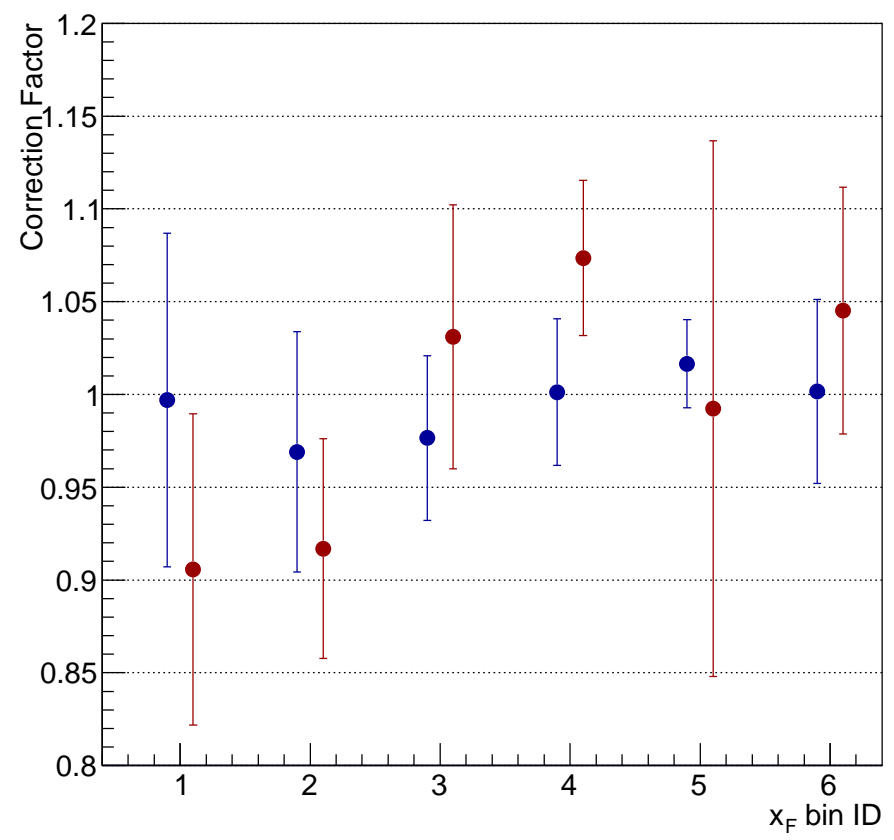

Figure 3.35: Correction factor $F_{A / C}^{R D}$ for Run-II . The blue solid circles are the ones for Fe/C and the red solid circles are for $\mathrm{W} / \mathrm{C}$.

\begin{tabular}{lccc}
$\mathbf{F e} / \mathbf{C}$ & \multicolumn{3}{c}{} \\
\hline$x_{F}$ bin & $F_{F e / C}^{R D}$ & $\delta F_{F e / C}^{s y s-R D f i t}$ & $\delta F_{F e / C}^{\text {sys-RI }}$ \\
\hline $0.00<x_{F} \leq 0.30$ & 0.997 & 0.036 & 0.082 \\
$0.30<x_{F} \leq 0.41$ & 0.969 & 0.050 & 0.041 \\
$0.41<x_{F} \leq 0.50$ & 0.977 & 0.037 & 0.025 \\
$0.50<x_{F} \leq 0.60$ & 1.001 & 0.033 & 0.022 \\
$0.60<x_{F} \leq 0.67$ & 1.017 & 0.019 & 0.014 \\
$0.67<x_{F} \leq 1.00$ & 1.002 & 0.029 & 0.041 \\
\hline & & & \\
$\mathbf{W} / \mathbf{C}$ & & & \\
\hline$x_{F}$ bin & $F_{W / C}^{R D}$ & $\delta F_{W / C}^{s y s-R D f i t}$ & $\delta F_{W / C}^{s y s-R I}$ \\
\hline $0.00<x_{F} \leq 0.30$ & 0.906 & 0.051 & 0.066 \\
$0.30<x_{F} \leq 0.41$ & 0.917 & 0.048 & 0.034 \\
$0.41<x_{F} \leq 0.50$ & 1.031 & 0.061 & 0.036 \\
$0.50<x_{F} \leq 0.60$ & 1.074 & 0.027 & 0.032 \\
$0.60<x_{F} \leq 0.67$ & 0.992 & 0.144 & 0.011 \\
$0.67<x_{F} \leq 1.00$ & 1.045 & 0.061 & 0.027 \\
\hline
\end{tabular}

Table 3.24: Correction factor $F_{A / C}^{R D}$ for Run-II . 
Roadset 62

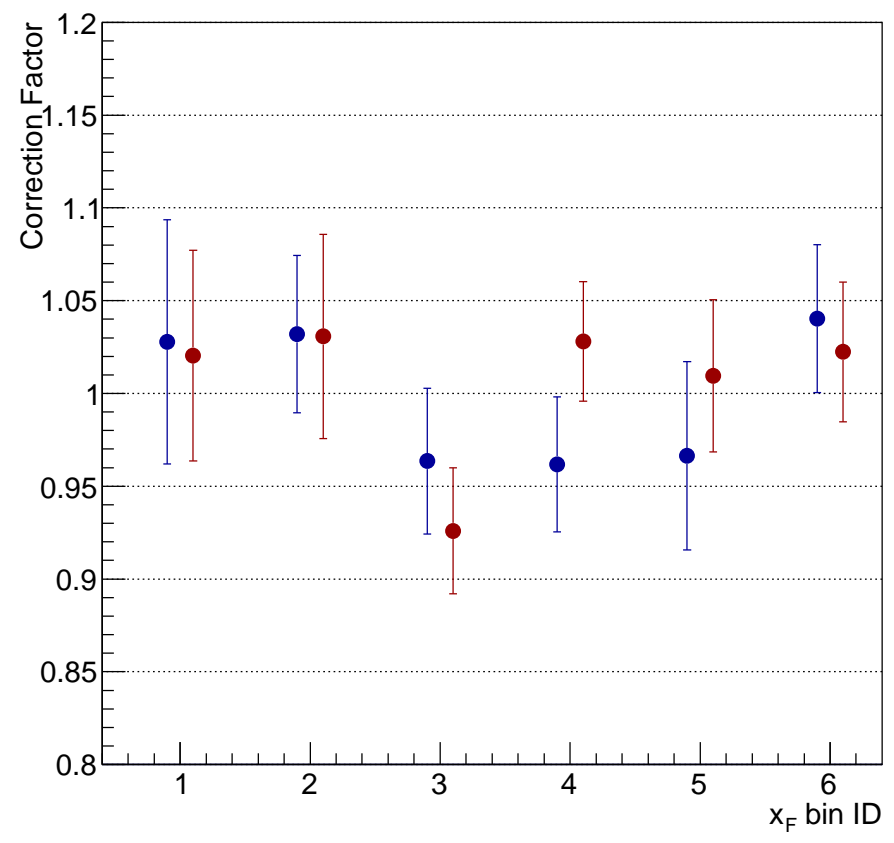

Figure 3.36: Correction factor $F_{A / C}^{R D}$ for Roadset 62 . The blue solid circles are the ones for Fe/C and the red solid circles are for $\mathrm{W} / \mathrm{C}$.

\begin{tabular}{lccc}
$\mathbf{F e} / \mathbf{C}$ & \multicolumn{3}{c}{} \\
\hline$x_{F}$ bin & $F_{F e / C}^{R D}$ & $\delta F_{F e / C}^{\text {sys-RDfit }}$ & $\delta F_{F e / C}^{\text {sys-RD }}$ \\
\hline $0.00<x_{F} \leq 0.30$ & 1.028 & 0.063 & 0.020 \\
$0.30<x_{F} \leq 0.41$ & 1.032 & 0.040 & 0.013 \\
$0.41<x_{F} \leq 0.50$ & 0.964 & 0.037 & 0.012 \\
$0.50<x_{F} \leq 0.60$ & 0.962 & 0.034 & 0.013 \\
$0.60<x_{F} \leq 0.67$ & 0.966 & 0.050 & 0.008 \\
$0.67<x_{F} \leq 1.00$ & 1.040 & 0.039 & 0.008 \\
\hline & & & \\
$\mathbf{W} / \mathbf{C}$ & & & \\
\hline$x_{F}$ bin & $F_{W / C}^{R D}$ & $\delta F_{W / C}^{s y s-R D f i t}$ & $\delta F_{W / C}^{s y s-R D T}$ \\
\hline $0.00<x_{F} \leq 0.30$ & 1.020 & 0.051 & 0.025 \\
$0.30<x_{F} \leq 0.41$ & 1.031 & 0.052 & 0.018 \\
$0.41<x_{F} \leq 0.50$ & 0.926 & 0.032 & 0.011 \\
$0.50<x_{F} \leq 0.60$ & 1.028 & 0.029 & 0.014 \\
$0.60<x_{F} \leq 0.67$ & 1.009 & 0.040 & 0.008 \\
$0.67<x_{F} \leq 1.00$ & 1.022 & 0.036 & 0.010 \\
\hline
\end{tabular}

Table 3.25: Correction $F_{A / C}^{R D}$ factor for Roadset 62 . 
Roadset 67

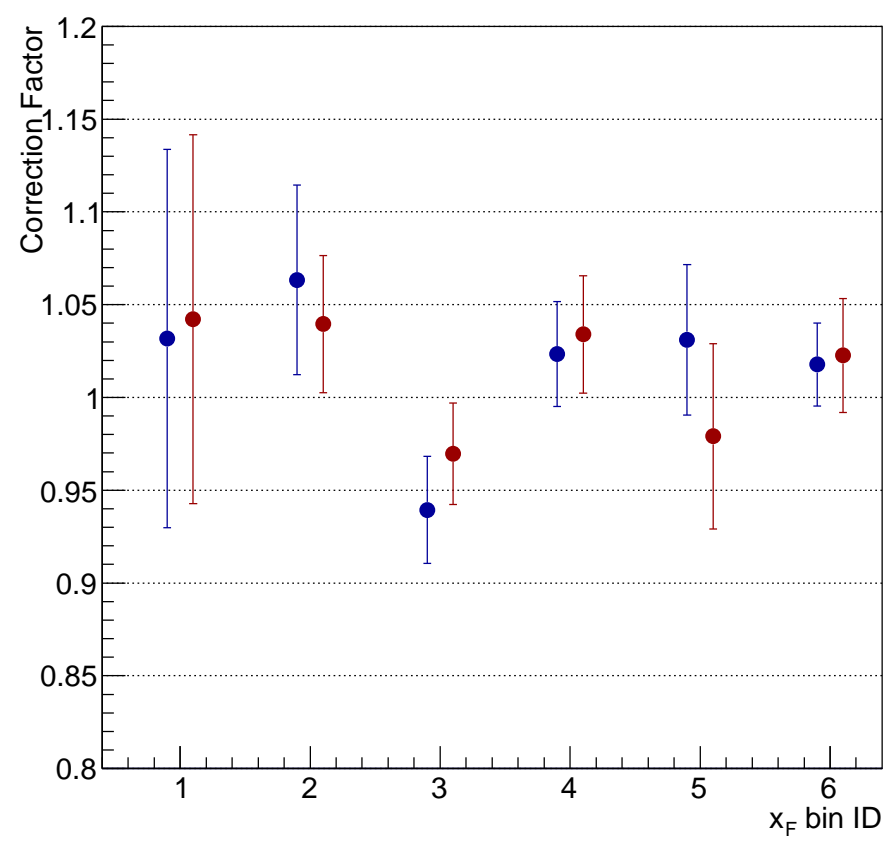

Figure 3.37: Correction factor $F_{A / C}^{R D}$ for Roadset 67 . The blue solid circles are the ones for Fe/C and the red solid circles are for $\mathrm{W} / \mathrm{C}$.

\begin{tabular}{lccc}
$\mathbf{F e} / \mathbf{C}$ & \multicolumn{3}{c}{} \\
\hline$x_{F}$ bin & $F_{F e / C}^{R D}$ & $\delta F_{F e / C}^{\text {sys-RDfit }}$ & $\delta F_{F e / C}^{\text {sys }-R D T}$ \\
\hline $0.00<x_{F} \leq 0.30$ & 1.032 & 0.095 & 0.036 \\
$0.30<x_{F} \leq 0.41$ & 1.063 & 0.046 & 0.023 \\
$0.41<x_{F} \leq 0.50$ & 0.939 & 0.025 & 0.014 \\
$0.50<x_{F} \leq 0.60$ & 1.023 & 0.024 & 0.015 \\
$0.60<x_{F} \leq 0.67$ & 1.031 & 0.037 & 0.017 \\
$0.67<x_{F} \leq 1.00$ & 1.018 & 0.011 & 0.020 \\
\hline & & & \\
$\mathbf{W} / \mathbf{C}$ & & & \\
\hline$x_{F}$ bin & $F_{W / C}^{R D}$ & $\delta F_{W / C}^{s y s-R D f i t}$ & $\delta F_{W / C}^{s y s-R D T}$ \\
\hline $0.00<x_{F} \leq 0.30$ & 1.042 & 0.093 & 0.034 \\
$0.30<x_{F} \leq 0.41$ & 1.040 & 0.028 & 0.024 \\
$0.41<x_{F} \leq 0.50$ & 0.970 & 0.022 & 0.017 \\
$0.50<x_{F} \leq 0.60$ & 1.034 & 0.027 & 0.017 \\
$0.60<x_{F} \leq 0.67$ & 0.979 & 0.047 & 0.016 \\
$0.67<x_{F} \leq 1.00$ & 1.023 & 0.021 & 0.022 \\
\hline
\end{tabular}

Table 3.26: Correction for $F_{A / C}^{R D}$ for Roadset 67. 


\subsubsection{Remaining Rate Dependence}

So far, only the rate dependence of the reconstruction efficiency, which is the rate dependence on the software level, has been considered. There can be other remaining rate-dependence effects such as the ones on the hardware level.

As to the hodoscopes, the rate dependence can originate from the voltage drop of the phototube voltage divider caused by the high intensity beam, and therefore leads to the low detection efficiency. As discussed before in the hodoscope section of the experimental setup, this effect should be mitigated with the new PMT base and could only cause negligible impact.

For the drift chambers, a source of rate-dependent chamber performance is related to the fact that the large number of tracks produced by a high intensity beam would generate a good amount of ions drifting in the chamber. These ions would then be collected by the high voltage wires and produce currents through the wires. Since the wires are connected to the so called "current-limiting resistor", which are used to protect the sense wires from breaking, caused by a sudden high current due to sparks, the ion-produced current can effectively lower the voltage difference between the sense wires and the cathode wires. In SeaQuest, the voltage drop, estimated based on a rough observation of the current drawn by a chamber over the time scale of a spill, is around the order of 10 volts in general. Due to the fluctuating intensity of the beam received by SeaQuest, the voltages supplied to the chamber planes are chosen to be around the edge of the plateau of efficiency versus voltage, in order to prevent possible damage with unexpected high intensity beam. Therefore, even a drop of voltage of few volts could noticeably reduce the efficiency of the chamber.

Before the start of Run- $\mathrm{V}$, there is not yet a well-established way to determine and correct the hardware-level rate dependence. A few hardware upgrades have been made such as probing the current through the wires in the chamber in some buckets before the triggering bucket so that the voltage drop and the effect on efficiency can be estimated. At this point, with limited knowledge on the remaining rate dependence, we would examine how much $R_{p A}$ can be affected.

To do such an estimation, the dimuon yield per trigger proton, corrected by $C^{c o r}$ already, 


\begin{tabular}{lcc}
\hline Data set & $\delta R_{\mathrm{Fe} / \mathrm{C}}^{\text {sys-RDemain }}$ & $\delta R_{W / C}^{\text {sys-RDremain }}$ \\
\hline Run-II & 0.051 & 0.038 \\
Roadset 62 & 0.044 & 0.046 \\
Roadset 67 & 0.019 & 0.027 \\
\hline
\end{tabular}

Table 3.27: Estimated systematic error from remaining rate dependence.

versus chamber intensity $I^{C}$ is made first. Since $C^{c o r}$ noticeably depends on $x_{F}$, the correction is made event by event depending on the corresponding $x_{F}$. Then we take the ratio of the yield of Fe to that of $\mathrm{C}$, and also $\mathrm{W}$ to $\mathrm{C}$. The results of the corrected-yield ratio are shown in Fig. 3.38, If the rate dependence is ideally corrected, the distribution of the ratio should be close to a flat line. In the case that the rate dependence is not fully amended, if the ratio is still flat, the results of the cross-section ratio measurements should not be affected by the still-existing rate dependence.

In Fig. 3.38, each ratio is fitted by a constant function, which is shown as the dashed line, and a linear function that is represented by the solid line. As can be seen, the overall distribution is nearly flat for both Roadset 62 and Roadset 67, and the ratio acquired from Roadset 62 shows a more significant deviation from a flat line. However, the slope of the linear fitting function is basically consistent with zero with the fitting error taken into account. These results indicate that even with the remaining rate dependence has only minimal effect the $R_{p A}$ measurements. Further attempts on correcting the remaining rate dependence is therefore neglected in this analysis. For now, a systematic error $\delta R_{A / C}^{\text {sys-RDremain }}$ is assigned to $R_{p A}$ to account for the uncertainty brought by the remaining rate dependence. The value of $\delta R_{A / C}^{s y s-R D r e m a i n}$ is derived as the standard deviation of the correct-yield-ratio values from different $I^{C}$ bins, and the result is summarized in Tab. 3.27 . 

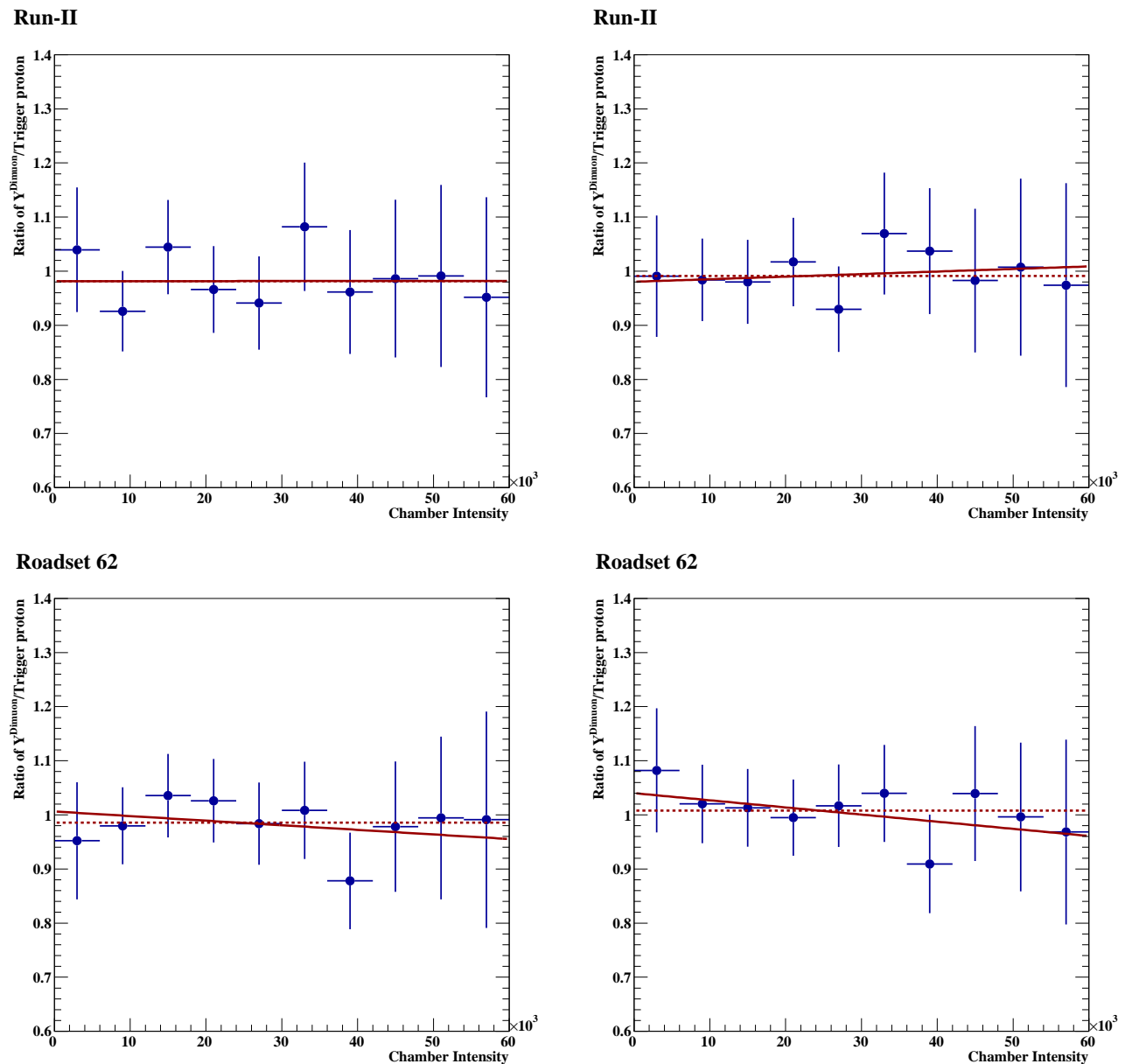

Roadset 62
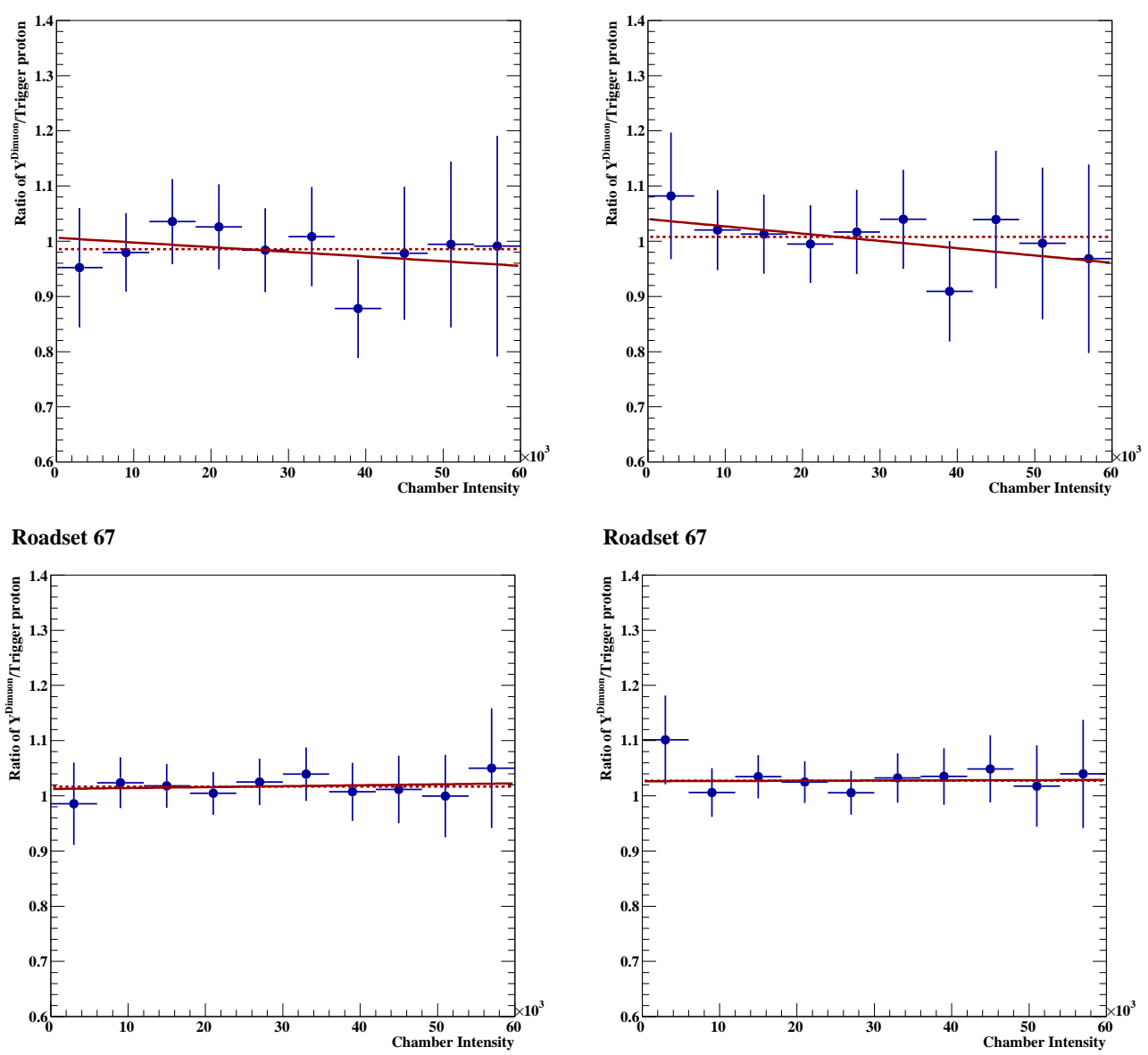

Figure 3.38: Corrected ratio of dimuon yield per trigger proton for each data set. The left plot is $\mathrm{Fe} / \mathrm{C}$ and the right plot is $\mathrm{W} / \mathrm{C}$. The result of the constant fit is shown by the dashed line, and the result of the linear fit is shown by the solid line. The rather large error bar is due to the incorporation of the uncertainty of the rate-dependence correction. 


\subsection{Detector Acceptance}

The last piece in Eq. 3.2 for the cross-section ratio derivation is the acceptance correction

factor $F_{A / C}^{A c p}$. In a broad definition, the detector acceptance of an experiment, $\mathcal{A}$, can be written as

$$
\mathcal{A}=\frac{N_{\text {reg }}}{N_{\text {phy }}}
$$

where $N_{\text {phys }}$ is the total number of physics events generated, and $N_{\text {reg }}$ denotes the ones among them that are actually registered by the detector. This acceptance definition accounts for any effect that can induce loses of events and is a convolution of the detector efficiency and finite geometric acceptability. Different experiments, can have their own exact definitions of acceptance. In most cases, $\mathcal{A}$ is a function of multiple kinematic variables, and determined by Monte Carlo simulations as the fraction of the accepted events with respect to the number of events generated.

To obtain the true yield of a physical process, the acceptance effect needs to be corrected by dividing the registered yield with $\mathcal{A}$, which is a critical step for the evaluation of absolute cross sections. For a cross-section-ratio measurement focused in this analysis, the quantity of concern is the ratio of acceptance between data of different targets, which is how the correction factor $F_{A / C}^{A c p}$ is defined, as

$$
F_{A / C}^{A c p}=\frac{\mathcal{A}_{C}}{\mathcal{A}_{A}}
$$

A significant advantage of ratio measurements is that with proper experimental design, the acceptance values between targets are basically identical with each other and $F_{A / C}^{A c p}$ is consequently unity. In SeaQuest, the short time-scale detector efficiency is correlated with the rate dependence and has already been discussed. As to the long time-sclae efficiency issues, such as the effect brought by an aged PMT in the hodoscope detector or a borken wire in the chamber, these inefficiencies can affect the overall trigger and tracking efficiency, reduce the Drell-Yan yield, and be mapped to the inefficiencies in specific kinematic regions. Nevertheless, the long time-scale inefficiencies are expected to be common to all targets and are therefore cancelled out in the ratio measurement with target rotation. As to the geometric acceptance, since all the solid targets are set up as three thin 
disks of the same diameter placed at identical $\mathrm{Z}$ positions along the beam line, the possible target dependence that can come from the variation of interaction position is thus negligible. Therefore, the acceptance correction factor $F_{A / C}^{A c p}$ can be simply treated as unity, which is confirmed by the analysis in [116]. It is true that for a detailed study, the acceptance correction factor should be more carefully evaluated with a Monte Carlo simulation that has proper nuclear-dependence effects

implemented. At this point, since the nuclear effects are still being investigated in SeaQuest, $F_{A / C}^{A c p}$ is adopted as one in this preliminary study.

\subsection{Dependency on Other Kinematic Variables}

The procedures for $R_{p A}\left(x_{F}\right)$ evaluation in this analysis have all been discussed in the previous sections. In this section, we consider the correlation of $R_{p A}\left(x_{F}\right)$ with other kinematic variables and explore how the results of energy loss determination can be influenced by other nuclear medium effects.

\subsection{1 $\quad p_{T}$ Dependence}

Other than the longitudinal kinematic variables, such as $x_{F}$ discussed in this study, the energy loss of partons can also be investigated via the nuclear dependence of dimuon transeverse momentum, $p_{T}$. The first clear experimental evidence that showed this nuclear dependence with the Drell-Yan process was observed by the NA10 collaboration [117. By comparing the cross section of massive dimuon production from tungsten to that from the deuterium, it was found the cross-section ratio is suppressed at low $p_{T}$ and gradually increases with $p_{T}$, which leads to a non-neglegible difference in the mean transverse momentum squared, $\left\langle p_{T}^{2}\right\rangle$. This phenomenon is usually denoted as the " $p_{T}$ broadening" and has been pointed out as an independent way to measure the radiative energy loss by references such as [17, 118]. Nevertheless, $p_{T}$ broadening is expected to be also contributed by the so-called "Cronin effect" [119], which is generally attributed to the transverse-momentum gain of a primary parton through multiple scatterings with partons from the heavy nucleus. The energy loss extraction by $\Delta\left\langle p_{T}^{2}\right\rangle$ requires a detailed independent study 


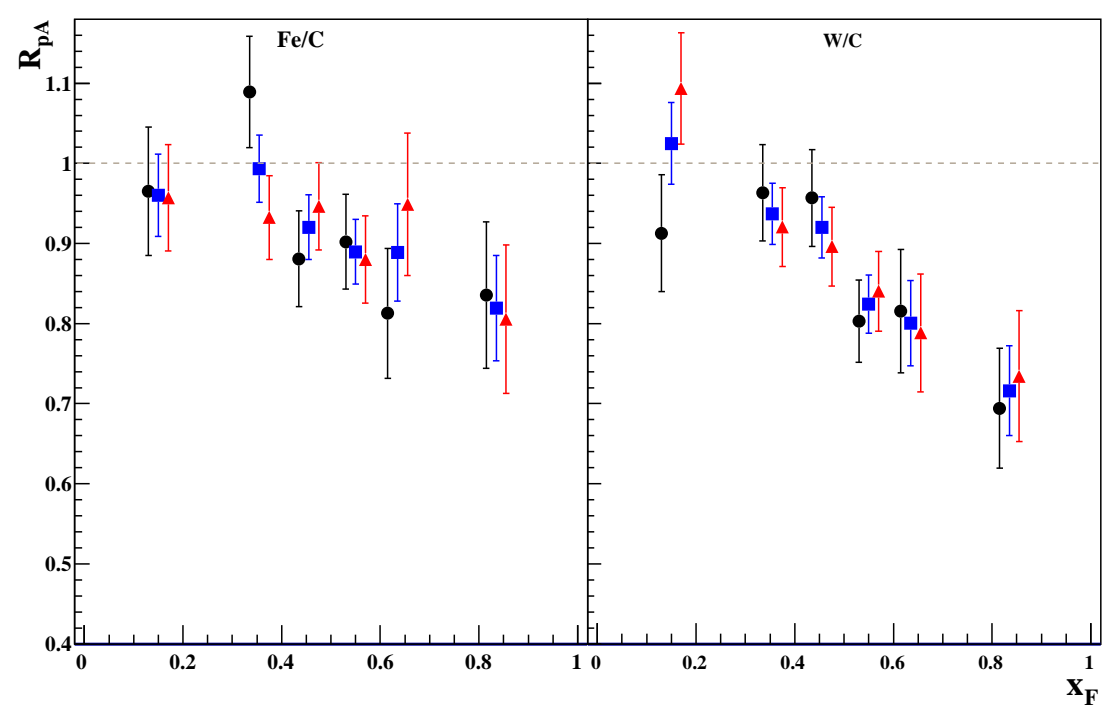

Figure 3.39: $p_{T}$ dependence of the Roadset-67 normalized yield ratios. The black circles are made with data of $p_{T}<0.6 \mathrm{GeV}$, red triangles are $p_{T} \geq 0.6 \mathrm{GeV}$, and blue squares are produced without making the $p_{T}$ selection.

which is not included in this work. Here, only the correlation between $x_{F}$ and $p_{T}$ is checked.

As given by Tab. 3.17, 3.18, and 3.19, the small variation of $\left\langle p_{T}\right\rangle$ values from different $x_{F}$ bins suggests that the $p_{T}$ values of the accepted events are nearly independent of $x_{F}$ and the behavior of $x_{F}$ distribution is decoupled from $p_{T}$. The Roadset-67 normalized yield ratios, identical to the ones discussed in Section 3.6.4, in different $p_{T}$ regions, $p_{T}<0.6 \mathrm{GeV}$ and $p_{T} \geq 0.6 \mathrm{GeV}$, are evaluated as an additional quick check. Together with the ratio including all $p_{T}$, the comparsion of yield ratios is shown in Fig. 3.39. With the agreement between ratios observed, it is concluded that the possible suppression at high $x_{F}$ has no apparent $p_{T}$ dependence.

\subsection{2 $x_{2}$ Dependence}

As discussed in the introduction chapter, the $x_{2}$ dependence is related to the effects on the modification of parton distribution in nuclear medium, which can generate a suppression in high$x_{F}$ as well. Referring to Tab. 3.17, 3.18, and 3.19 once again, one can see the clear anti-correlation between $x_{2}$ and $x_{F}$; the average $x_{2}$ drops with increasing $x_{F}$, which is anticipated for SeaQuest 

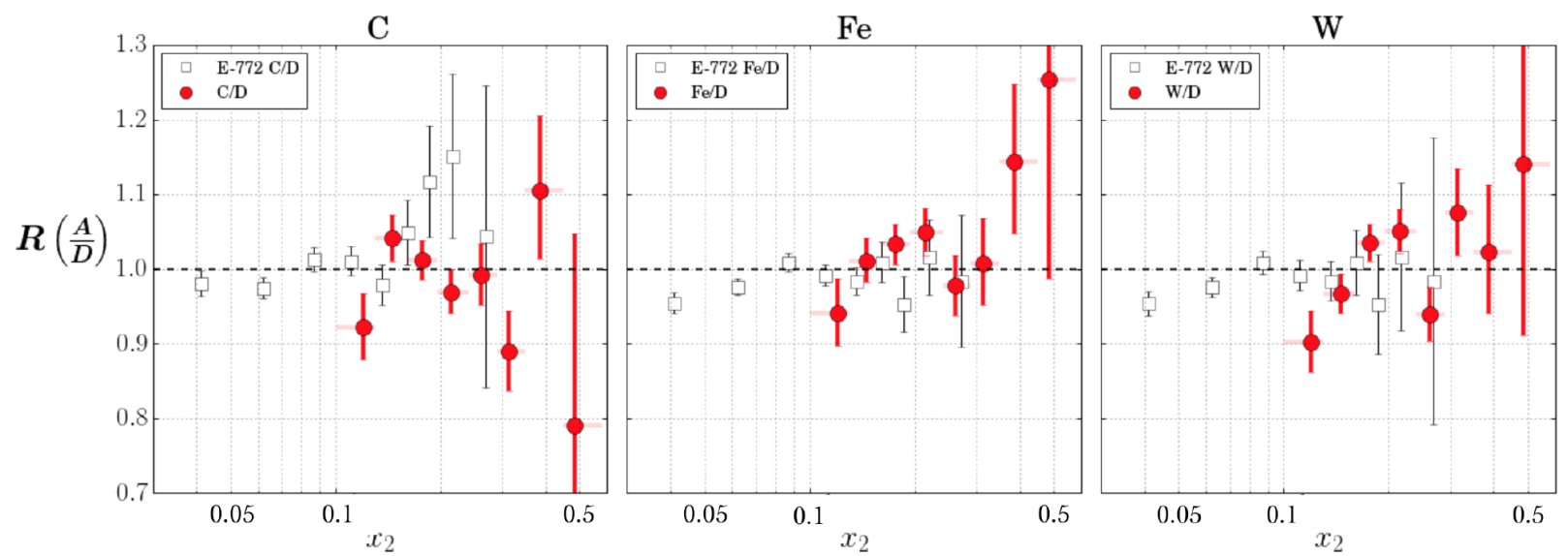

Figure 3.40: The E906 per-nucleon cross section ratios versus $x_{2}$ acquired by Bryan Dannowitz, together with the data from E772 [116]. The E906 result is given as the red solid circles and the E772 one as the open squares. Only the statistical errors are shown.

with a forward-acceptance spectrometer. The high- $x_{F}$ suppression can thus be correlated to the low $x_{2}$ nuclear shadowing. Nevertheless, even for the highest $x_{F}$ bin, the average $x_{2}$ is approximately 0.14 in general, which is still well above the range of strong shadowing effect.

In the independent preliminary study of Bryan Dannowitz, the collaborator from the University of Illonois [116], the nuclear dependence of $R_{p A}$ as a function of $x_{2}$ is investigated and the results are shown in Fig. 3.40, As illustrated by the plots in Fig. 3.40, only slight shadowing effect has been observed in the kinematic coverage of SeaQuest, similar to the E772 measurement. In addition, the effects from anti-shadowing and EMC are not clearly seen and the $R_{p A}$ measured are consistent with 1 in higher $x_{2}$ region. Based on the results of the $R_{p A}$ versus $x_{2}$ measurements from SeaQuest and also E772, requiring $x_{2}>0.15$ is sufficient for excluding the shadowing effect, and the possible $x_{2}$ dependence from higher $x_{2}$ should not play a significant role in the energy loss measurement. In this analysis, the $R_{p A}\left(x_{F}\right)$ is also evaluated with the $x_{2}>0.15$ cut and the result will be compared with the one without this $x_{2}$ requirement. The corresponding plots and tables in deriving the $R_{p A}\left(x_{F}\right)$ of $x_{2}>0.15$ are given in Appendix $\mathrm{C}$. 


\section{Chapter 4}

\section{Results and Comparison}

\subsection{Results of Cross-section Ratios as a Function of $x_{F}$}

As discussed in the previous chapter, the $R_{p A}\left(x_{F}\right)$ defined in Eq. 3.2 consists of three major pieces: the normalized yield ratio $R_{Y i e l d}$, the rate-dependence correction factor $F_{A / C}^{R D}$, and the acceptance-correction factor $F_{A / C}^{A c p}$. The $R_{Y i e l d}$ is demonstrated in Section 3.6.4, the $F_{A / C}^{R D}$ is available in Section 3.7.3, and the $F_{A / C}^{A c p}$ is treated as unity, as explained in Section 3.8. Putting all the ingredients together, $R_{p A}\left(x_{F}\right)$ can be derived as

$$
R_{p A}\left(x_{F}\right)=R_{Y i e l d}\left(x_{F}\right) \cdot F_{A / C}^{R D}\left(x_{F}\right) \cdot 1
$$

With the $F_{A / C}^{R D}$ corrections being applied on $R_{Y i e l d}$, the $R_{p A}\left(x_{F}\right)$ results from each data set are shown in Fig. 4.1. The $R_{p A}$ values of $\mathrm{Fe} / \mathrm{C}$ and $\mathrm{W} / \mathrm{C}$, together with the corresponding errors, are listed in Tab. 4.1, 4.2, and 4.3, where the $\sigma_{\text {stat }}$ is the statistical error, $\sigma_{\text {sys }}^{B G f i t}$ denotes the systematic error originating from the mass fitting for $F_{b k g}$ extraction, $\sigma_{s y s}^{R D f i t}$ comes from the fitting error of the rate-dependent reconstruction efficiency, $\sigma_{\text {sys }}^{R D I}$ corresponds to the error brought by the uncertainty in chamber intensity of the Drell-Yan events, and $\sigma_{\text {sys }}^{\text {RDremain }}$ is the error estimated for the remaining rate-dependence. 


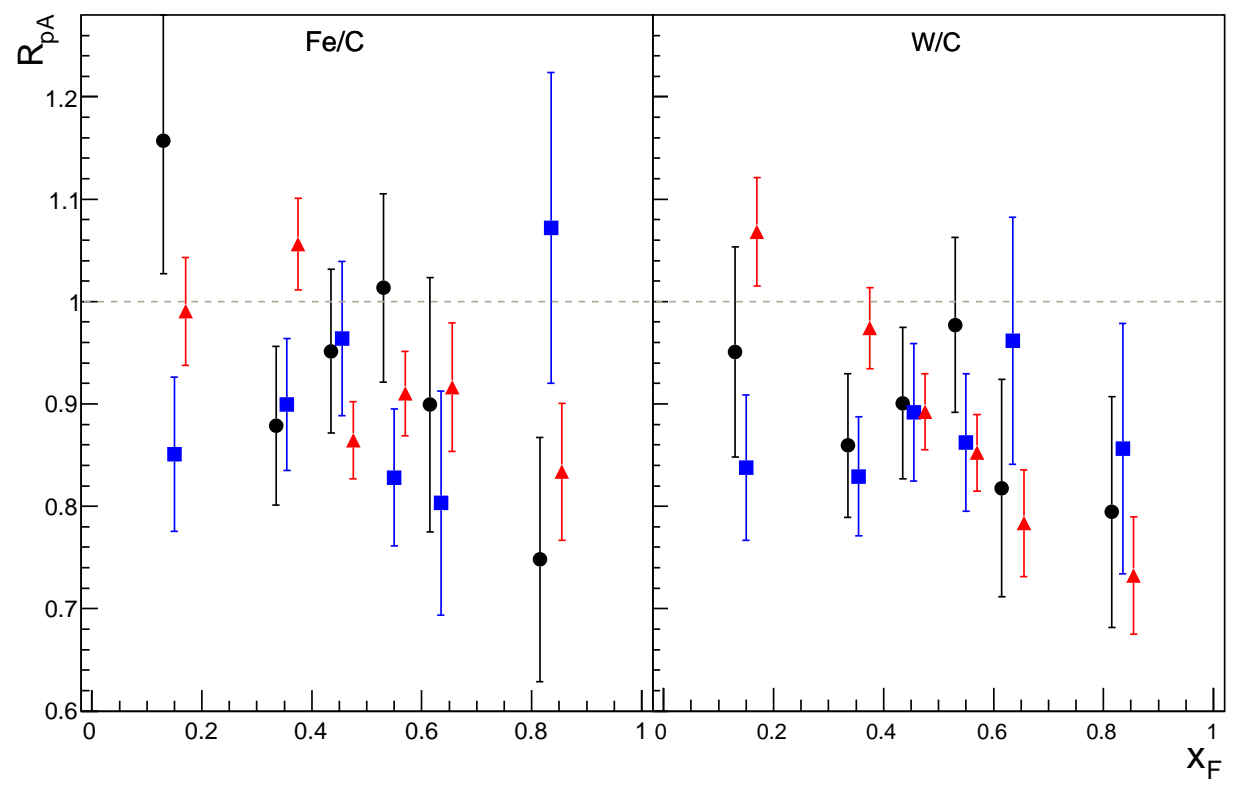

Figure 4.1: $R_{p A}\left(x_{F}\right)$ acquired from each dataset. Only the statistical errors are shown. The black circles are results of Run-II , the blue squares are of Roadset 62, and red triangles are of Roadset 67 .

\begin{tabular}{lcccccc}
$\mathbf{F e} / \mathbf{C}$ & \multicolumn{7}{c}{} \\
\hline$x_{F}$ bin & $R_{p A}$ & $\sigma_{\text {stat }}$ & $\sigma_{\text {sys }}^{\text {BGfit }}$ & $\sigma_{\text {sys }}^{\text {RDit }}$ & $\sigma_{\text {sys }}^{\text {RDI }}$ & $\sigma_{\text {sys }}^{\text {RDemain }}$ \\
\hline $0.00<x_{F} \leq 0.30$ & 1.157 & 0.130 & 0.007 & 0.042 & 0.095 & 0.059 \\
$0.00<x_{F} \leq 0.30$ & 0.879 & 0.078 & 0.005 & 0.044 & 0.036 & 0.045 \\
$0.00<x_{F} \leq 0.30$ & 0.951 & 0.080 & 0.004 & 0.035 & 0.024 & 0.048 \\
$0.00<x_{F} \leq 0.30$ & 1.013 & 0.092 & 0.006 & 0.034 & 0.022 & 0.051 \\
$0.00<x_{F} \leq 0.30$ & 0.899 & 0.124 & 0.006 & 0.017 & 0.013 & 0.046 \\
$0.00<x_{F} \leq 0.30$ & 0.748 & 0.119 & 0.009 & 0.021 & 0.030 & 0.038 \\
\hline
\end{tabular}

\begin{tabular}{lcccccc}
$\mathbf{W} / \mathbf{C}$ & \multicolumn{7}{l}{} \\
\hline$x_{F}$ bin & $R_{p A}$ & $\sigma_{\text {stat }}$ & $\sigma_{\text {sys }}^{\text {BGfit }}$ & $\sigma_{\text {sys }}^{\text {RDit }}$ & $\sigma_{\text {sys }}^{\text {RDI }}$ & $\sigma_{\text {sys }}^{\text {RDemain }}$ \\
\hline $0.30<x_{F} \leq 0.41$ & 0.951 & 0.102 & 0.006 & 0.049 & 0.063 & 0.036 \\
$0.30<x_{F} \leq 0.41$ & 0.859 & 0.070 & 0.005 & 0.041 & 0.029 & 0.033 \\
$0.30<x_{F} \leq 0.41$ & 0.901 & 0.074 & 0.005 & 0.055 & 0.032 & 0.034 \\
$0.30<x_{F} \leq 0.41$ & 0.977 & 0.086 & 0.006 & 0.026 & 0.032 & 0.037 \\
$0.30<x_{F} \leq 0.41$ & 0.818 & 0.106 & 0.004 & 0.118 & 0.009 & 0.031 \\
$0.30<x_{F} \leq 0.41$ & 0.795 & 0.113 & 0.007 & 0.048 & 0.021 & 0.030 \\
\hline
\end{tabular}

Table 4.1: $R_{p A}$ values and the corresponding errors for Run-II . 


\begin{tabular}{lcccccc}
$\mathbf{F e} / \mathbf{C}$ & \multicolumn{7}{c}{} \\
\hline$x_{F}$ bin & $R_{p A}$ & $\sigma_{\text {stat }}$ & $\sigma_{\text {sys }}^{\text {BGfit }}$ & $\sigma_{\text {sys }}^{\text {RDit }}$ & $\sigma_{\text {sys }}^{\text {RDI }}$ & $\sigma_{\text {sys }}^{\text {RDemain }}$ \\
\hline $0.00<x_{F} \leq 0.30$ & 0.851 & 0.075 & 0.003 & 0.053 & 0.017 & 0.037 \\
$0.00<x_{F} \leq 0.30$ & 0.899 & 0.065 & 0.004 & 0.036 & 0.012 & 0.040 \\
$0.00<x_{F} \leq 0.30$ & 0.964 & 0.075 & 0.005 & 0.036 & 0.012 & 0.042 \\
$0.00<x_{F} \leq 0.30$ & 0.828 & 0.067 & 0.004 & 0.028 & 0.011 & 0.036 \\
$0.00<x_{F} \leq 0.30$ & 0.803 & 0.109 & 0.006 & 0.040 & 0.007 & 0.035 \\
$0.00<x_{F} \leq 0.30$ & 1.072 & 0.152 & 0.010 & 0.042 & 0.008 & 0.047 \\
\hline
\end{tabular}

\begin{tabular}{lcccccc}
$\mathbf{W} / \mathbf{C}$ & \multicolumn{7}{l}{} \\
\hline$x_{F}$ bin & $R_{p A}$ & $\sigma_{\text {stat }}$ & $\sigma_{\text {sys }}^{\text {BGfit }}$ & $\sigma_{\text {sys }}^{\text {RDit }}$ & $\sigma_{\text {sys }}^{\text {RDI }}$ & $\sigma_{\text {sys }}^{\text {RDemain }}$ \\
\hline $0.30<x_{F} \leq 0.41$ & 0.838 & 0.071 & 0.003 & 0.043 & 0.021 & 0.039 \\
$0.30<x_{F} \leq 0.41$ & 0.829 & 0.058 & 0.004 & 0.043 & 0.015 & 0.038 \\
$0.30<x_{F} \leq 0.41$ & 0.892 & 0.067 & 0.005 & 0.028 & 0.010 & 0.041 \\
$0.30<x_{F} \leq 0.41$ & 0.862 & 0.067 & 0.005 & 0.025 & 0.012 & 0.040 \\
$0.30<x_{F} \leq 0.41$ & 0.962 & 0.120 & 0.007 & 0.039 & 0.008 & 0.045 \\
$0.30<x_{F} \leq 0.41$ & 0.856 & 0.122 & 0.010 & 0.031 & 0.009 & 0.040 \\
\hline
\end{tabular}

Table 4.2: $R_{p A}$ values and the corresponding errors for Roadset 62 .

\begin{tabular}{lcccccc}
$\mathbf{F e} / \mathbf{C}$ & \multicolumn{7}{c}{} \\
\hline$x_{F}$ bin & $R_{p A}$ & $\sigma_{\text {stat }}$ & $\sigma_{\text {sys }}^{\text {BGfit }}$ & $\sigma_{\text {sys }}^{\text {RDit }}$ & $\sigma_{\text {sys }}^{\text {RDI }}$ & $\sigma_{\text {sys }}^{\text {RDemain }}$ \\
\hline $0.00<x_{F} \leq 0.30$ & 0.991 & 0.053 & 0.003 & 0.094 & 0.036 & 0.019 \\
$0.00<x_{F} \leq 0.30$ & 1.056 & 0.045 & 0.003 & 0.048 & 0.024 & 0.020 \\
$0.00<x_{F} \leq 0.30$ & 0.865 & 0.038 & 0.002 & 0.022 & 0.012 & 0.016 \\
$0.00<x_{F} \leq 0.30$ & 0.910 & 0.041 & 0.003 & 0.022 & 0.014 & 0.017 \\
$0.00<x_{F} \leq 0.30$ & 0.916 & 0.063 & 0.003 & 0.034 & 0.015 & 0.017 \\
$0.00<x_{F} \leq 0.30$ & 0.834 & 0.067 & 0.005 & 0.009 & 0.016 & 0.016 \\
\hline
\end{tabular}

\begin{tabular}{lcccccc}
$\mathbf{W} / \mathbf{C}$ & \multicolumn{7}{l}{} \\
\hline$x_{F}$ bin & $R_{p A}$ & $\sigma_{\text {stat }}$ & $\sigma_{\text {sys }}^{\text {BGfit }}$ & $\sigma_{\text {sys }}^{\text {RDit }}$ & $\sigma_{\text {sys }}^{\text {RDI }}$ & $\sigma_{\text {sys }}^{\text {RDemain }}$ \\
\hline $0.30<x_{F} \leq 0.41$ & 1.068 & 0.053 & 0.003 & 0.100 & 0.036 & 0.029 \\
$0.30<x_{F} \leq 0.41$ & 0.974 & 0.040 & 0.003 & 0.027 & 0.023 & 0.027 \\
$0.30<x_{F} \leq 0.41$ & 0.892 & 0.037 & 0.003 & 0.019 & 0.015 & 0.024 \\
$0.30<x_{F} \leq 0.41$ & 0.852 & 0.037 & 0.003 & 0.023 & 0.015 & 0.023 \\
$0.30<x_{F} \leq 0.41$ & 0.784 & 0.052 & 0.003 & 0.037 & 0.012 & 0.021 \\
$0.30<x_{F} \leq 0.41$ & 0.732 & 0.057 & 0.005 & 0.015 & 0.016 & 0.020 \\
\hline
\end{tabular}

Table 4.3: $R_{p A}$ values and the corresponding errors for Roadset 67 . 
To combine the results from each data set, the conventional method of using the inverse of the error squared as the weighting is adopted. The procedures are as the follows:

- For each data set $s$, the individual systematic errors are combined to give the overall systematic error as $\sigma_{\text {sys }}^{s}=\sqrt{\sum_{i}\left(\sigma_{\text {sys }}^{i}\right)^{2}}$.

- Take the sum of the statistical and systematic error squared, and use the inverse of the sum, $1 /\left[\left(\sigma_{\text {stat }}^{s}\right)^{2}+\left(\sigma_{\text {sys }}^{s}\right)^{2}\right]$, as the weighting to evaluate the combined cross-section ratio $\overline{R_{p A}}$.

- Combine the statistic (systematic) error from each data set to get the overall statistical (systematic) error as $\sigma_{\text {stat }(\text { sys })}=\sqrt{\frac{1}{\sum_{s} 1 /\left(\sigma_{\text {stat }(\text { sys })}\right)^{2}}}$.

- The combined cross-section ratio is expressed as: $\overline{R_{p A}} \pm \sigma_{\text {stat }} \pm \sigma_{\text {sys }}$.

The way to determine the $x_{F}$ value and its corresponding uncertainty of the combined $R_{p A}$ data point in each $x_{F}$ bin is described as follows. As illustrated in Fig. 3.25, it has been shown that the measured $x_{F}$ values in different data sets are close to each other. The $x_{F}$ distributions from all data sets are merged together first. Since there is no significant difference of the mean $x_{F}$ measured between carbon and the other solid targets, which can be seen in Tab. 3.17, 3.18, and 3.19 , in each $x_{F}$ bin of the merged $x_{F}$ distribution, the mean $x_{F}$ value of the iron (tungsten) data in this bin is adopted as the $x_{F}$ of the $R_{p A}$ data point of $\mathrm{Fe} / \mathrm{C}(\mathrm{W} / \mathrm{C}$ ). The lower (upper) uncertainty is subsequently determined as the lower (upper) bound of the interval between the boundary and the mean $x_{F}$ in which $68 \%$ of all the data below (above) the mean $x_{F}$ is included.

The final combined results of $R_{p A}\left(x_{F}\right)$ are given in Fig. 4.2, and summarized in Tab. 4.4. As illustrated in the plots, a clear decreasing behavior in $R_{p A}$ with increasing $x_{F}$ is observed in both the $\mathrm{Fe} / \mathrm{C}$ and $\mathrm{W} / \mathrm{C}$ results. In the region of high $x_{F}$, the $R_{p A}$ values are significantly smaller than unity, and the $R_{p A}\left(x_{F}\right)$ of $\mathrm{W} / \mathrm{C}$ shows a stronger suppression than the one of $\mathrm{Fe} / \mathrm{C}$. 


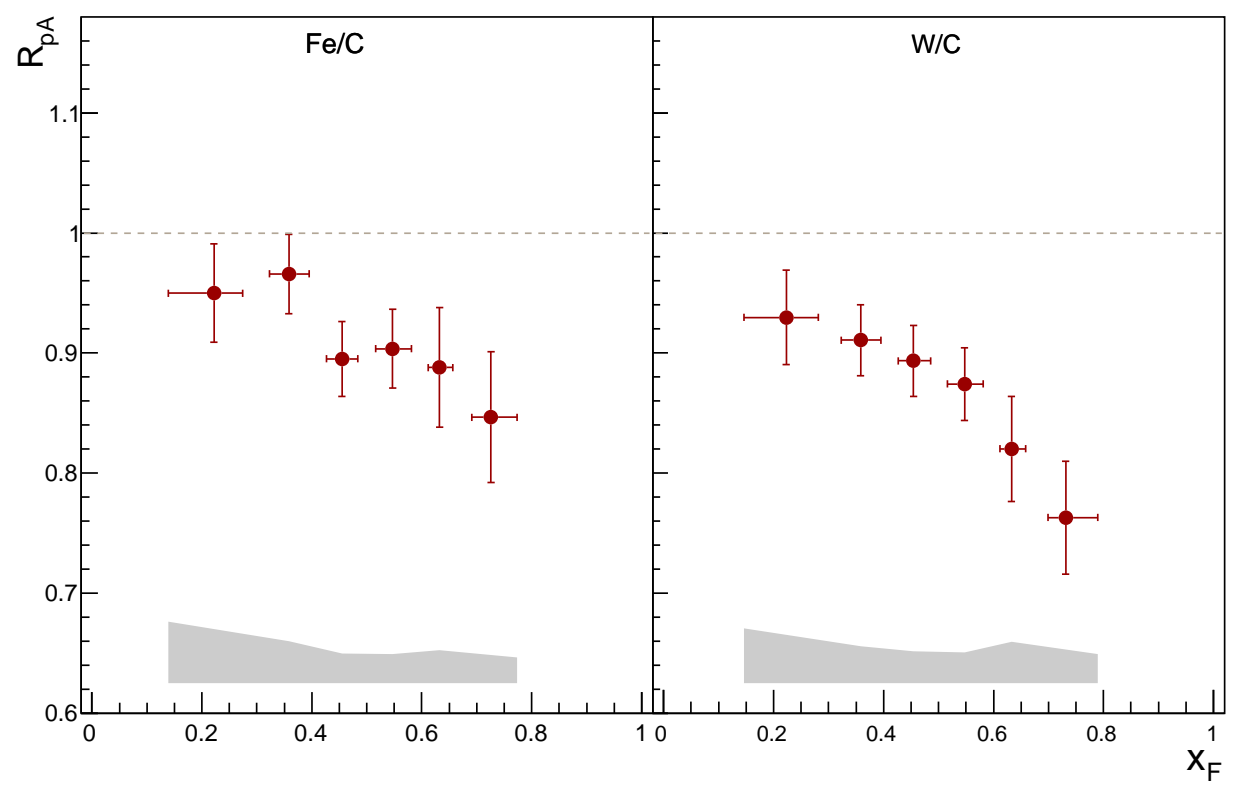

Figure 4.2: The combined $R_{p A}\left(x_{F}\right)$ results. The statistical errors are plotted along with the data points and the corresponding systematic errors are presented as the grey band.

\begin{tabular}{llll}
$\mathbf{F e} / \mathbf{C}$ & & & \\
\hline$x_{F}$ & $\overline{R_{p A}}$ & $\pm \sigma_{\text {stat }}$ & $\pm \sigma_{\text {sys }}$ \\
\hline $0.22[+0.05,-0.08]$ & 0.950 & \pm 0.041 & \pm 0.051 \\
$0.36[+0.04,-0.03]$ & 0.966 & \pm 0.033 & \pm 0.035 \\
$0.45[+0.03,-0.03]$ & 0.895 & \pm 0.031 & \pm 0.025 \\
$0.55[+0.03,-0.03]$ & 0.904 & \pm 0.033 & \pm 0.024 \\
$0.63[+0.02,-0.02]$ & 0.888 & \pm 0.050 & \pm 0.028 \\
$0.73[+0.05,-0.04]$ & 0.847 & \pm 0.054 & \pm 0.021 \\
\hline
\end{tabular}

\begin{tabular}{llll}
$\mathbf{W} / \mathbf{C}$ & & & \\
\hline$x_{F}$ & $\overline{R_{p A}}$ & $\pm \sigma_{\text {stat }}$ & $\pm \sigma_{\text {sys }}$ \\
\hline $0.22[+0.06,-0.08]$ & 0.930 & \pm 0.039 & \pm 0.046 \\
$0.36[+0.04,-0.04]$ & 0.911 & \pm 0.030 & \pm 0.031 \\
$0.45[+0.03,-0.03]$ & 0.893 & \pm 0.030 & \pm 0.027 \\
$0.55[+0.03,-0.03]$ & 0.874 & \pm 0.030 & \pm 0.026 \\
$0.63[+0.03,-0.02]$ & 0.820 & \pm 0.044 & \pm 0.034 \\
$0.73[+0.06,-0.03]$ & 0.763 & \pm 0.047 & \pm 0.024 \\
\hline
\end{tabular}

Table 4.4: Combined $R_{p A}$ values and the corresponding errors. 


\subsection{Comparison}

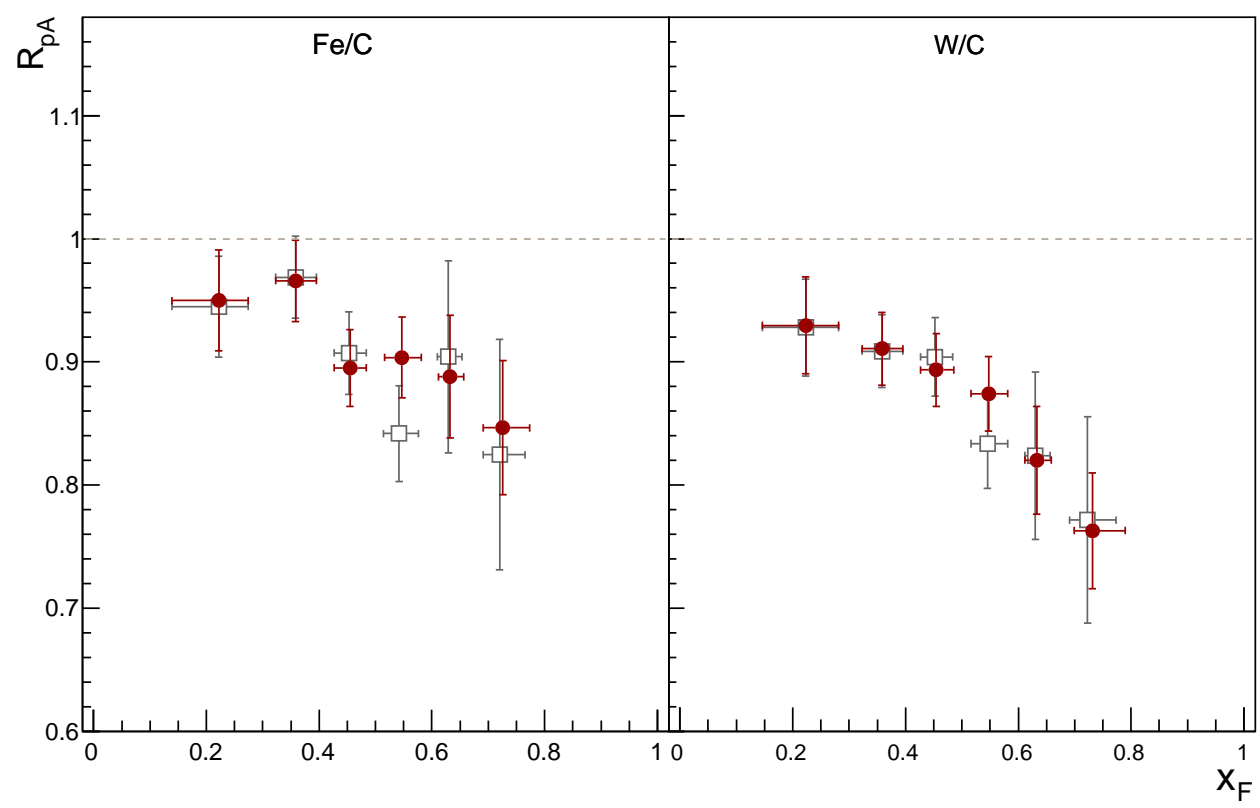

Figure 4.3: The combined $R_{p A}\left(x_{F}\right)$ results including the one with $x_{1}$ cut. The red solid circles are the results without $x_{1}$ cut. The gray open squares are the ones with $x_{2}>0.15$ cut. Only the statistical errors are presented.

In Fig. 4.3 the $R_{p A}\left(x_{F}\right)$ presented in the previous section is overlaid with the one evaluated with an additional $x_{2}>0.15$ cut enforced. As can be seen, the $R_{p A}$ values in small $x_{F}$ region with $x_{2}>0.15$ is in good agreement with the one without $x_{2}$ cut. This is consistent with the fact that $x_{2}$ and $x_{F}$ are anti-correlated and manipulating the data in the small $x_{2}$ region has more significant effect on higher $x_{F}$. In the high- $x_{F}$ region, in spite of the larger statistical error, the $R_{p A}\left(x_{F}\right)$ with the $x_{2}$ cut shows a descending pattern similar to the one without the cut. As indicated in Section 3.9.2, requiring $x_{2}$ to be greater than 0.15 should efficiently remove the contribution from the shadowing effect. The overall consistency between the two $R_{p A}\left(x_{F}\right)$ distribution suggests the negligible nuclear shadowing in the Drell-Yan events collected in SeaQuest, which is what has been anticipated. The apparent $R_{p A}$ attenuation with $x_{F}$ in both cases is evidence for the presence of the energy loss of the incident beam quark. The stronger degree of suppression in the $R_{p A}\left(x_{F}\right)$ of 
$\mathrm{W} / \mathrm{C}$ indicates a larger energy loss in the heavier tungsten target as expected.

The measured no- $x_{2}$-cut $R_{p A}\left(x_{F}\right)$ is compared with the theoretical prediction given by Neufeld et al. [100, as shown in Fig. 4.4. In their calculation, the initial-state radiative energy loss based on the DGLV approach [120] is considered. The energy loss is linearly dependent on the energy of the parton, and the energy loss rate (also denoted as the stopping power in the work), $d E / d x$. is parameterized as

$$
-d E / d x=E / X_{0}
$$

where $X_{0}$ is the quark radiation length. The nuclear shadowing is included in the numerical result by employing the EKS98 shadowing parameterization [84. From Fig. 4.4, one can notice that the $R_{p A}$ values of Fe/C roughly follows the line predicted with $X_{0}=50 \mathrm{fm}$ while the ones of $\mathrm{W} / \mathrm{C}$ lies above this prediction in general. This inconsistency, although not significant with the corresponding error being considered, might be a global normalization issue or could be attributed to the nPDF parameterization, which has not yet been well determined in the kinematic region involved. Despite this overall discrepancy, the general trend of suppression matches the theoretical estimation. Based on the measurement so far, the uncertainty of the $R_{p A}$ is rather large for a precise determination of the radiation length. Nevertheless, it is likely that the $X_{0}$ falls into the range between $50 \mathrm{fm}$ and $160 \mathrm{fm}$, which is consistent with the value of the order of $50 \mathrm{fm}$ to $100 \mathrm{fm}$, as extracted with the E772/E866 data [100]. To have a rough idea about the scale of energy loss, take the $\left\langle x_{1}\right\rangle$ of the Drell-Yan events collected, which is $\sim 0.64$, the average incoming quark energy can be derived as $76.8 \mathrm{GeV}$ and $\langle-d E / d x\rangle$ lies in the range of $\sim 0.77$ to $\sim 1.55 \mathrm{GeV} / \mathrm{fm}$, with $X_{0}$ being 50 fm to $100 \mathrm{fm}$.

In addition, the path-length dependence of energy loss effect is explored by checking the behavior of $R_{p A}$ at high $x_{F}$ versus the linear size of the nucleus, $\sim A^{1 / 3}$. By taking the $R_{p A}$ of the highest $x_{F}$ bin of $\mathrm{W} / \mathrm{C}$ and $\mathrm{Fe} / \mathrm{C}$, where the energy-loss suppression is most significant, the attenuation of $R_{p A}$ as a function of $A^{1 / 3}$ is plotted in Fig. 4.5 . In this figure, the carbon data point is simply set as one, and the uncertainty is taken as the $\sqrt{2}$ times the statistical error by convention. 

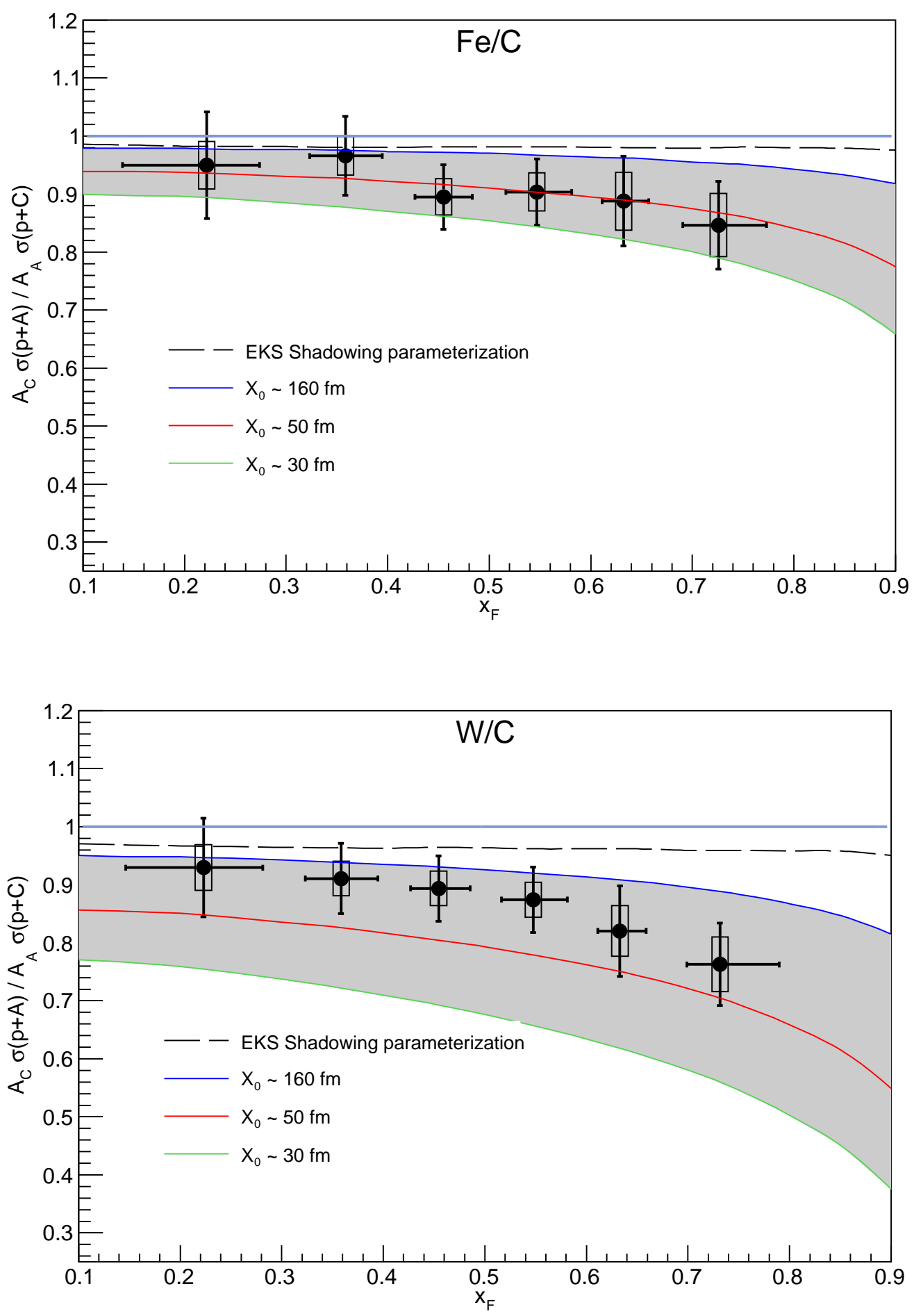

Figure 4.4: $R_{p A}\left(x_{F}\right)$ with the theoretic predictions given in [100]. The shadowing effect is included and the numerical results are estimated with three different radiation lengths, $X_{0}=30 \mathrm{fm}, 50$ $\mathrm{fm}$, and $160 \mathrm{fm}$. The grey bands indicate the regions where the E906 results are likely to be, as predicted by [100]. 


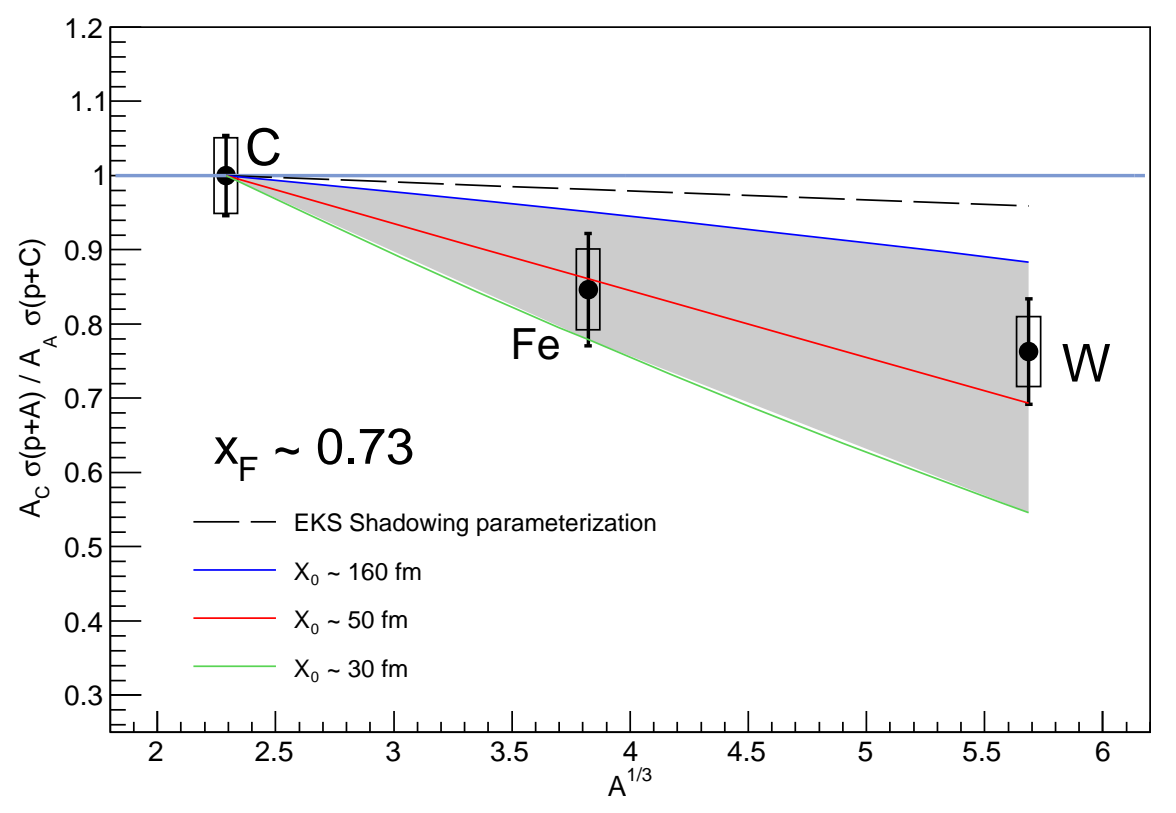

Figure 4.5: The $A^{1 / 3}$ dependence of energy loss effect. The linear $A^{1 / 3}$ dependence is given in [100]. The shadowing effect is included and the numerical results are estimated with three different radiation lengths, $X_{0}=30 \mathrm{fm}, 50 \mathrm{fm}$, and $160 \mathrm{fm}$.

As indicated by [100], if energy loss has a quadratic dependence on the path length, the $R_{p A}$ of $\mathrm{W} / \mathrm{C}$ would be significantly smaller than the value estimated based on a linear trend given by the carbon and iron data points. Fig. 4.5 shows that the result fits the description of linear path-length dependence, as given by Neufeld et al.. Although with only three data points currently available, a solid conclusion cannot yet be made. With a future analysis that evaluates the cross-section ratio between the solid targets and deuterium, an additional data point can be used to further constrain the $A^{1 / 3}$ dependency of energy loss. 


\section{Chapter 5}

\section{Summary and Future Prospects}

E906/SeaQuest is an experiment emphasizing the measurement of high-mass dimuon events produced by the Drell-Yan process or meson decay. With $120 \mathrm{GeV}$ from the Fermilab Main Injector incident on fixed targets of liquid hydrogen, liquid deuterium, carbon, iron, and tungsten, SeaQuest has been collecting analyzable data since late 2013. In this work, approximately $1 / 3$ of the estimated total data was analyzed.

With minimal final-state interactions, the Drell-Yan process is regarded as an ideal probe of parton energy loss. In this thesis, the energy loss of the incident quark was studied by measuring the per-nucleon Drell-Yan cross section ratio, $R_{p A}\left(x_{F}\right)$, of the heavy nuclear targets, iron and tungsten, to carbon. In extracting the yield of Drell-Yan events, the main contribution of background was identified as dimuon pairs formed by erroneously-combined uncorrelated muons. By randomly mixing single-muon tracks, this combinatorial background could be successfully reproduced and thus subtracted. Another issue for the $R_{p A}$ determination was the dependency on proton beam intensity, which was denoted as the rate dependence. The rate-dependence of the dimuon reconstruction efficiency was investigated by mixing clean Monte Carlo events with the random-trigger data, and the corresponding correction was made.

In both the $\mathrm{Fe} / \mathrm{C}$ and $\mathrm{W} / \mathrm{C}$ results, the measured $R_{p A}\left(x_{F}\right)$ shows a clear attenuation trend with increasing $x_{F}$, with $R_{p A}$ at high- $x_{F}$ being significantly smaller than unity. With the $x_{2}>$ 0.15 cut applied, the resulting $R_{p A}\left(x_{F}\right)$ was not noticeably different with the one without the $x_{2}$

requirement. This consistency of $R_{p A}\left(x_{F}\right)$ indicates negligible nuclear shadowing contributions and 
the observed suppression in $R_{p A}\left(x_{F}\right)$ is largely from energy loss. The $R_{p A}\left(x_{F}\right)$ result was compared with the theoretical prediction calculated by Neufeld et al. [121]. Limited by the uncertainty, the quark radiation length, $X_{0}$, was unable to be precisely determined at this point. Nevertheless, the result suggested that $X_{0}$ should lie in the range of $50 \mathrm{fm}$ to $160 \mathrm{fm}$, which agrees with the value of $50 \mathrm{fm}$ to $160 \mathrm{fm}$ derived by using the E772/E866 data. Additionally, the $R_{p A}$ values at high $x_{F}$ as a function of $A^{1 / 3}$ suggests a possible linear path-length dependence of energy loss, but needs to be confirmed with the inclusion of deuterium-target data.

The preliminary results in this study demonstrate the effect from quark energy loss in the kinematic range that should not be strongly affected by nuclear shadowing. Recently, an improved version of the tracking program has been developed, in which the overall tracking efficiency is increased and the rate-dependent inefficiency is expected to be mitigated by about $10 \%$. The latest data production processed with this tracker will be available within a few months. In addition, improved event-selection cuts and a new chamber-occupancy based approach to the rate-dependence analysis are being developed. The results reported here will be re-evaluated with an analysis employing these updated resources and cross checked independently before being released as SeaQuest official results.

The SeaQuest collaboration is putting great effort to have the rest of data processed and analyzed with improved tracking and selection cuts. By including higher statistics data, the statistical error is expected to be reduced by approximately a factor of $\sqrt{3}$. Furthermore, the systematic error could be brought down by by the ability to investigate hardware-level rate dependence in the latest dataset. With the upcoming high-precision result, the quark energy loss can be well determined and used to distinguish the predictions from different theoretical models.

Another opportunity for measuring quark energy loss in cold nuclear matter is provided by the Japan Proton Accelerator Research Complex (J-PARC). By utilizing the 50 GeV (currently at $30 \mathrm{GeV}$ ) proton beam from the J-PARC main ring, a similar energy-loss measurement with Drell-Yan process can be carried out. With the lower proton-beam energy compared to SeaQuest, a steeper attenuation of $R_{p A}$ at high $x_{F}$ induced by energy loss effect is expected. In addition, 
the energy loss has also been proposed as an interesting topic to be studied at the upcoming Electron Ion Collider (EIC) [121]. Rather than Drell-Yan, hadron production via semi-inclusive deep-inelastic process (SIDIS) is employed to investigate the final-state energy loss of the parton produced. With a wider kinematic coverage that can be offered by EIC, more stringent tests on the theoretical models can be applied. Together with the measurements from high-energy collisions, the experimental possibilities, combined with various theoretical efforts, are delineating a promising future in which a detailed knowledge of parton energy loss can be achieved. 


\section{Bibliography}

[1] M. Gell-Mann, Phys. Lett. 8, 214 (1964).

[2] G. Zweig, in DEVELOPMENTS IN THE QUARK THEORY OF HADRONS. VOL. 1. 1964 - 1978, edited by D. Lichtenberg and S. P. Rosen (1964), pp. 22-101.

[3] H. Fritzsch, M. Gell-Mann, and H. Leutwyler, Phys. Lett. B 47, 365 (1973).

[4] C. N. Yang and R. L. Mills, Phys. Rev. 96, 191 (1954).

[5] H. D. Politzer, Phys. Rev. Lett. 30, 1346 (1973).

[6] D. J. Gross and F. Wilczek, Phys. Rev. Lett. 30, 1343 (1973).

[7] J. C. Collins, D. E. Soper, and G. F. Sterman, Nucl. Phys. B 261, 104 (1985).

[8] J. C. Collins, D. E. Soper, and G. F. Sterman, Adv. Ser. Direct. High Energy Phys. 5, 1 (1989).

[9] Y. Dokshitzer, Sov. Phys. JETP. 46, 641 (1977).

[10] V. N. Gribov and L. N. Lipatov, Sov. J. Nucl. Phys. 15, 438 (1972), [Yad. Fiz.15,781(1972)].

[11] G. Altarelli and G. Parisi, Nucl. Phys. B 126, 298 (1977).

[12] T. Falter, W. Cassing, K. Gallmeister, and U. Mosel, Phys. Rev. C 70, 054609 (2004).

[13] G.-Y. Qin and X.-N. Wang, Int. J. Mod. Phys. E 24, 1530014 (2015), [,309(2016)].

[14] L. D. Landau and I. Pomeranchuk, Dokl. Akad. Nauk Ser. Fiz. 92, 535 (1953).

[15] A. B. Migdal, Phys. Rev. 103, 1811 (1956).

[16] R. Baier, Y. L. Dokshitzer, A. H. Mueller, S. Peigne, and D. Schiff, Nucl. Phys. B 483, 291 (1997).

[17] R. Baier, Y. L. Dokshitzer, A. H. Mueller, S. Peigne, and D. Schiff, Nucl. Phys. B 484, 265 (1997).

[18] B. Zakharov, JETP letters 63, 952 (1996).

[19] B. Zakharov, JETP letters 65, 615 (1997). 
[20] X.-N. Wang and M. Gyulassy, Phys. Rev. Lett. 68, 1480 (1992).

[21] M. Gyulassy and X.-N. Wang, Nucl. Phys. B 420, 583 (1994).

[22] R. Baier, Y. L. Dokshitzer, A. H. Mueller, and D. Schiff, Nucl. Phys. B 531, 403 (1998).

[23] U. A. Wiedemann, Nucl. Phys. B 588, 303 (2000).

[24] U. A. Wiedemann, Nucl. Phys. A 690, 731 (2001).

[25] C. A. Salgado and U. A. Wiedemann, Phys. Rev. D 68, 014008 (2003).

[26] N. Armesto, C. A. Salgado, and U. A. Wiedemann, Phys. Rev. D 69, 114003 (2004).

[27] M. Gyulassy, P. Levai, and I. Vitev, Nucl. Phys. B 571, 197 (2000).

[28] M. Gyulassy, P. Levai, and I. Vitev, Phys. Rev. Lett. 85, 5535 (2000).

[29] M. Gyulassy, P. Lévai, and I. Vitev, Nucl. Phys. B 594, 371 (2001).

[30] M. Djordjevic and M. Gyulassy, Nucl. Phys. A 733, 265 (2004).

[31] S. Wicks, W. Horowitz, M. Djordjevic, and M. Gyulassy, Nucl. Phys. A 784, 426 (2007).

[32] M. Djordjevic and U. Heinz, Phys. Rev. Lett. 101, 022302 (2008).

[33] P. Arnold, G. D. Moore, and L. G. Yaffe, J. High Energy Phys. 2001, 057 (2001).

[34] P. Arnold, G. D. Moore, and L. G. Yaffe, J. High Energy Phys. 2001, 009 (2001).

[35] P. Arnold, G. D. Moore, and L. G. Yaffe, J. High Energy Phys. 2002, 030 (2002).

[36] S. Caron-Huot and C. Gale, Phys. Rev. C 82, 064902 (2010).

[37] X. Guo and X.-N. Wang, Phys. Rev. Lett. 85, 3591 (2000).

[38] X.-N. Wang and X. Guo, Nucl. Phys. A 696, 788 (2001).

[39] A. Majumder, Phys. Rev. D 85, 014023 (2012).

[40] N. Armesto, B. Cole, C. Gale, W. A. Horowitz, P. Jacobs, S. Jeon, M. van Leeuwen, A. Majumder, B. Müller, G.-Y. Qin, et al., Phys. Rev. C 86, 064904 (2012).

[41] Y. Mehtar-Tani, C. A. Salgado, and K. Tywoniuk, Phys. Rev. Lett. 106, 122002 (2011).

[42] N. Armesto, H. Ma, Y. Mehtar-Tani, C. A. Salgado, and K. Tywoniuk, JHEP 01, 109 (2012).

[43] G.-Y. Qin, J. Ruppert, C. Gale, S. Jeon, G. D. Moore, and M. G. Mustafa, Phys. Rev. Lett. 100, 072301 (2008).

[44] J. D. Bjorken, FERMILAB-PUB-82-059-THY (1982).

[45] S. Cao, G.-Y. Qin, and S. A. Bass, Phys. Rev. C 88, 044907 (2013).

[46] D. Sharma (PHENIX), J. Phys. G 38, 124082 (2011). 
[47] R. A. Bertens (ALICE), in Proceedings, 50th Rencontres de Moriond, QCD and high energy interactions: La Thuile, Italy, March 21-28, 2015 (2015), pp. 263-266.

[48] A. Airapetian et al. (HERMES), Nucl. Phys. B 780, 1 (2007).

[49] W. K. Brooks and H. Hakobyan, Nucl. Phys. A 830, 361C (2009).

[50] A. Accardi, Acta Phys. Hung. A 27, 189 (2006).

[51] N. Akopov, L. Grigoryan, and Z. Akopov, Eur. Phys. J. C 70, 5 (2010).

[52] B. Z. Kopeliovich, J. Nemchik, I. K. Potashnikova, and I. Schmidt, Phys. Rev. C 86, 054904 (2012).

[53] G. T. Garvey and J. C. Peng, Phys. Rev. Lett. 90, 092302 (2003).

[54] C.-G. Duan, S.-W. Cui, and Z.-Y. Yan, Cummun. Theor. Phys. 44, 123 (2005).

[55] A. Accardi, F. Arleo, W. K. Brooks, D. D'Enterria, and V. Muccifora, Riv. Nuovo Cim. 32, 439 (2010).

[56] S. D. Drell and T.-M. Yan, Phys. Rev. Lett. 25, 316 (1970).

[57] J. H. Christenson, G. S. Hicks, L. M. Lederman, P. J. Limon, B. G. Pope, and E. Zavattini, Phys. Rev. Lett. 25, 1523 (1970).

[58] J. H. Christenson, G. S. Hicks, L. M. Lederman, P. J. Limon, B. G. Pope, and E. Zavattini, Phys. Rev. D 8, 2016 (1973).

[59] G. Altarelli, R. K. Ellis, and G. Martinelli, Nucl. Phys. B 143, 521 (1978), [Erratum: Nucl. Phys.B146,544(1978)].

[60] G. Altarelli, R. K. Ellis, and G. Martinelli, Nucl. Phys. B 157, 461 (1979).

[61] J. Kubar-Andre and F. E. Paige, Physical Review D 19, 221 (1979).

[62] T.-M. Yan, in Sid Drell Symposium Stanford, California, July 31, 1998 (1998).

[63] T. Matsuura, R. Hamberg, and W. L. van Neerven, Nucl. Phys. B 345, 331 (1990).

[64] R. Hamberg, W. L. van Neerven, and T. Matsuura, Nucl. Phys. B 359, 343 (1991), [Erratum: Nucl. Phys.B644,403(2002)].

[65] W. L. van Neerven and E. B. Zijlstra, Nucl. Phys. B 382, 11 (1992), [Erratum: Nucl. Phys.B680,513(2004)].

[66] Neural Network Parton Distribution Functions website, Online (Accessed: 2017.05.25), URL https://nnpdf.hepforge.org/.

[67] R. D. Ball et al. (NNPDF), JHEP 04, 040 (2015).

[68] MMHT Parton Distribution Functions website, Online (Accessed: 2017.05.25), URL http: //www.hep.ucl.ac.uk/mmht/index.shtml. 
[69] L. A. Harland-Lang, A. D. Martin, P. Motylinski, and R. S. Thorne, Eur. Phys. J. C 75, 204 (2015).

[70] CT14 Parton Distribution Functions website, Online (Accessed: 2017.05.25), URL http:// hep.pa.msu.edu/cteq/public/.

[71] S. Dulat, T.-J. Hou, J. Gao, M. Guzzi, J. Huston, P. Nadolsky, J. Pumplin, C. Schmidt, D. Stump, and C. P. Yuan, Phys. Rev. D 93, 033006 (2016).

[72] H1Zeus working group homepage, Online (Accessed: 2017.05.25), URL https://www-h1. desy.de/h1/www/publications/.

[73] H. Abramowicz et al. (ZEUS, H1), Eur. Phys. J. C 75, 580 (2015).

[74] CTEQ-Jefferson Lab Collaboration homepage, Online (Accessed: 2017.05.25), URL https: //www.jlab.org/theory/cj/.

[75] A. Accardi, L. T. Brady, W. Melnitchouk, J. F. Owens, and N. Sato, Phys. Rev. D 93, 114017 (2016).

[76] S. Alekhin, J. Bluemlein, S.-O. Moch, and R. Placakyte (2016).

[77] D. M. Alde, H. W. Baer, T. A. Carey, G. T. Garvey, A. Klein, C. Lee, M. J. Leitch, J. W. Lillberg, P. L. McGaughey, C. S. Mishra, et al., Phys. Rev. Lett. 64, 2479 (1990).

[78] J.-J. Aubert, G. Bassompierre, K. Becks, C. Best, E. Böhm, X. de Bouard, F. Brasse, C. Broll, S. Brown, J. Carr, et al., Physics Letters B 123, 275 (1983).

[79] K. Ackerstaff et al. (HERMES), Phys. Lett. B 475, 386 (2000), [Erratum: Phys. Lett.B567,339(2003)].

[80] S. Dasu et al., Phys. Rev. Lett. 60, 2591 (1988).

[81] J. Seely et al., Phys. Rev. Lett. 103, 202301 (2009).

[82] K. Rith, Subnucl. Ser. 51, 431 (2015).

[83] K. J. Eskola, V. J. Kolhinen, and P. V. Ruuskanen, Nucl. Phys. B 535, 351 (1998).

[84] K. J. Eskola, V. J. Kolhinen, and C. A. Salgado, Eur. Phys. J. C 9, 61 (1999).

[85] K. J. Eskola, V. J. Kolhinen, H. Paukkunen, and C. A. Salgado, JHEP 05, 002 (2007).

[86] D. de Florian and R. Sassot, Phys. Rev. D 69, 074028 (2004).

[87] M. Hirai, S. Kumano, and T.-H. Nagai, Phys. Rev. C 76, 065207 (2007).

[88] K. J. Eskola, H. Paukkunen, and C. A. Salgado, JHEP 04, 065 (2009).

[89] M. A. Vasiliev, M. E. Beddo, C. N. Brown, T. A. Carey, T. H. Chang, W. E. Cooper, C. A. Gagliardi, G. T. Garvey, D. F. Geesaman, E. A. Hawker, et al. (FNAL E866/NuSea), Phys. Rev. Lett. 83, 2304 (1999).

[90] S. Gavin and J. Milana, Phys. Rev. Lett. 68, 1834 (1992). 
[91] S. J. Brodsky and P. Hoyer, Phys. Lett. B 298, 165 (1993).

[92] R. Baier, Y. L. Dokshitzer, A. H. Mueller, S. Peigne, and D. Schiff, Nucl. Phys. B 484, 265 (1997).

[93] R. Baier, Y. L. Dokshitzer, A. H. Mueller, and D. Schiff, Nucl. Phys. B 531, 403 (1998).

[94] M. B. Johnson et al. (FNAL E772), Phys. Rev. Lett. 86, 4483 (2001).

[95] F. Arleo, Phys. Lett. B 532, 231 (2002).

[96] M. B. Johnson, B. Z. Kopeliovich, I. K. Potashnikova, P. L. McGaughey, J. M. Moss, J. C. Peng, G. Garvey, M. Leitch, C. N. Brown, and D. M. Kaplan, Phys. Rev. C 65, 025203 (2002).

[97] J. Arrington, D. Geesaman, K. Hafidi, R. Holt, H. Jackson, D. Potterveld, P. Reimer, P. Solvignon, C. Brown, C. Gagliardi, et al., SeaQuest proposal (2006).

[98] J. Badier et al. (NA3), Phys. Lett. 104B, 335 (1981), [,807(1981)].

[99] H. Xing, Y. Guo, E. Wang, and X.-N. Wang, Nucl. Phys. A 879, 77 (2012).

[100] R. Neufeld, I. Vitev, and B.-W. Zhang, Physics Letters B 704, 590 (2011).

[101] Fermilab's Accelerator Complex, Online (Accessed: 2017.03.01), URL https://www.fnal. gov/pub/science/particle-accelerators/.

[102] N. L. W.M. Bokhari, J.G. Heinrich and F. Newcomer, Nuclear Science Symposium, 1998 Conference Record 1, 445 (1998).

[103] C. Morris et al., Science and Global Security 16, 37 (2008).

[104] E. Jastrzembski, Trigger Supervisor v2 (TS), JLab (2015.05.22), URL https://coda.jlab. org/drupal/system/files/pdfs/HardwareManual/misc/TriggerSupervisorV2.pdf.

[105] R. E. McClellan, Ph.D. thesis, Illinois U., Urbana (2016).

[106] CODA Documentation website, Online (Accessed: 2017.03.01), URL https://coda.jlab. org/drupal/.

[107] L. R. Dalesio, J. O. Hill, M. Kraimer, S. Lewis, D. Murray, S. Hunt, W. Watson, M. Clausen, and J. Dalesio, Nucl. Instrum. Meth. A352, 179 (1994).

[108] R. E. Kalman et al., Phys. Rev. Lett. 82, 35 (1960).

[109] K. Liu, SeaQuest Internal Documentation DocDB-1283 (2015).

[110] Geant4 website, Online (Accessed: 2017.06.12), URL http://geant4.cern.ch/.

[111] S. Gorbunov and I. Kisel, CBM-SOFT-note-2006-001 (2006).

[112] K. Liu, SeaQuest Internal Documentation DocDB-1487 (2015). 
[113] G. Moreno, C. N. Brown, W. E. Cooper, D. Finley, Y. B. Hsiung, A. M. Jonckheere, H. Jostlein, D. M. Kaplan, L. M. Lederman, Y. Hemmi, et al., Phys. Rev. D 43, 2815 (1991).

[114] A. S. Ito, R. J. Fisk, H. Jöstlein, D. M. Kaplan, S. W. Herb, D. C. Hom, L. M. Lederman, H. D. Snyder, J. K. Yoh, B. C. Brown, et al., Phys. Rev. D 23, 604 (1981).

[115] B. Kerns, SeaQuest Internal Documentation DocDB-1460 (2015).

[116] B. P. Dannowitz, Ph.D. thesis, Illinois U., Urbana (2016).

[117] P. Bordalo et al. (NA10), Phys. Lett. B 193, 373 (1987).

[118] P. McGaughey, J. Moss, and J.-C. Peng, Annual Review of Nuclear and Particle Science 49, 217 (1999).

[119] J. W. Cronin, H. J. Frisch, M. J. Shochet, J. P. Boymond, P. A. Piroué, and R. L. Sumner, Phys. Rev. D 11, 3105 (1975).

[120] I. Vitev, Phys. Rev. C 75, 064906 (2007).

[121] A. Accardi et al., Eur. Phys. J. A 52, 268 (2016).

[122] R. E. McClellan, SeaQuest internal communications.

[123] Garfield - simulation of gaseous detectors, Online (Accessed: 2017.05.25), URL http:// garfield.web.cern.ch/garfield/

[124] F. Sauli, Tech. Rep., Cern (1977). 


\section{Appendix A}

\section{New Station-1 Drift Chamber}

The University of Colorado is the primary group responsible for the fabrication of the upgraded station-1 drift chamber, D1.2, for the SeaQuest experiment. Having a larger acceptance in the horizontal direction, the new station 1 chamber is the key factor to probing the high- $x_{2}$ region for the experiment and allows more high-mass events to be accepted, as shown in Fig. A.1. In this appendix, the details of the design and construction of the upgraded station-1 chamber is presented.
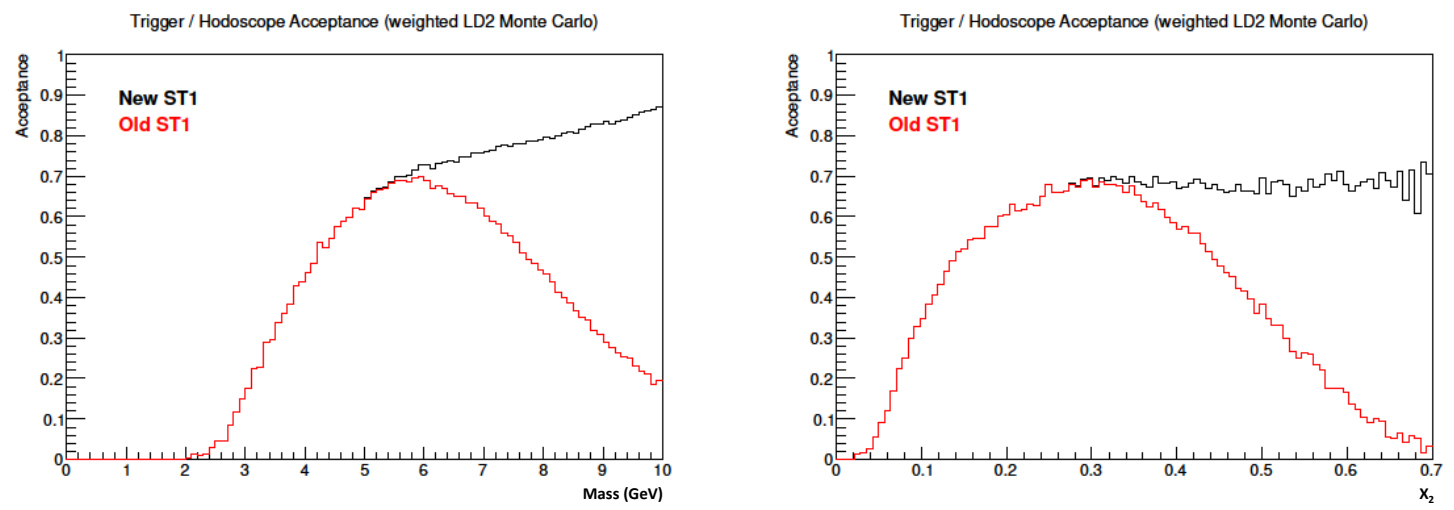

Figure A.1: Acceptance comparison between the old and new station-1 chambers [122]. The left (right) plot shows the acceptance as a function of mass $\left(x_{2}\right)$. The FPGA-1 trigger roads are required to be fired in the acceptance evaluation.

The new station-1 chamber consists of two main pieces: the wire frame and the gas box. The wire frame is an aluminum structure with which the wires are strung. The gas box is an airtight container that holds the wire frame in it, and is consistently flowed with specific gas mixture during operation. These two components are discussed separately in the following sections. 


\section{A.1 The Wire Frame}

The layout of the wire planes and the cell structure of the D1.2 are demonstrated in Fig. A.2 and Fig. A.3. The design of the drift cell is different from the one commonly adopted for drift chambers, in which additional cathode planes are implemented so that the cathode wires in a cell is always parallel to the anode wire. The position dependence of the electric field along the anode wire generated by this choice was studied by the Garfield simulation program [123, and the effect was shown to be acceptable under ordinary working conditions. The electric field and potential of a single cell simulated by Garfield are plotted in Fig. A.4. The wires used in both the cathode and the guard planes are gold-plated beryllium copper wires, and the anode planes utilize gold-plated tungsten wires. There are 640 wires in a cathode or guard plane, 320 wires in a X-view plane, and 384 wires in each of the U/V-view planes. Since there are two guard planes, seven cathode planes, and two anode planes in each view, a total of 7,936 wires were employed in the chamber. The specifications of the wires are listed in Tab. A.1.

A traditional way of wire stringing is to use a wire winding machine to position the wires

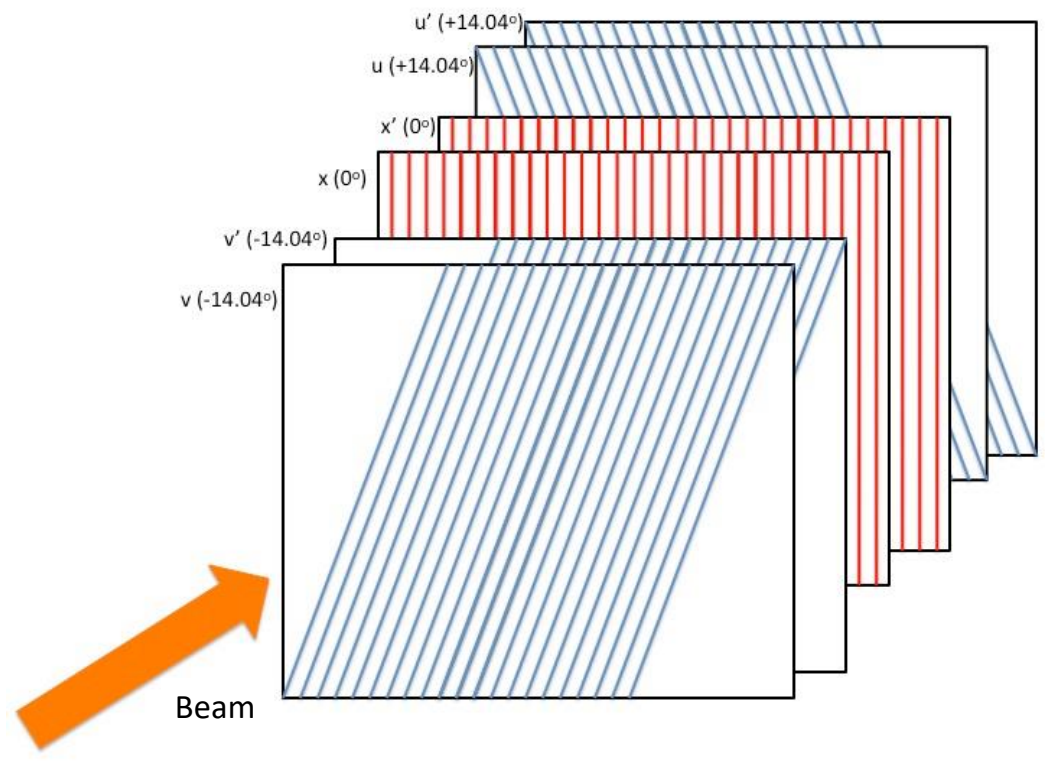

Figure A.2: Schematic of the layout of new Station-1 wire planes. 


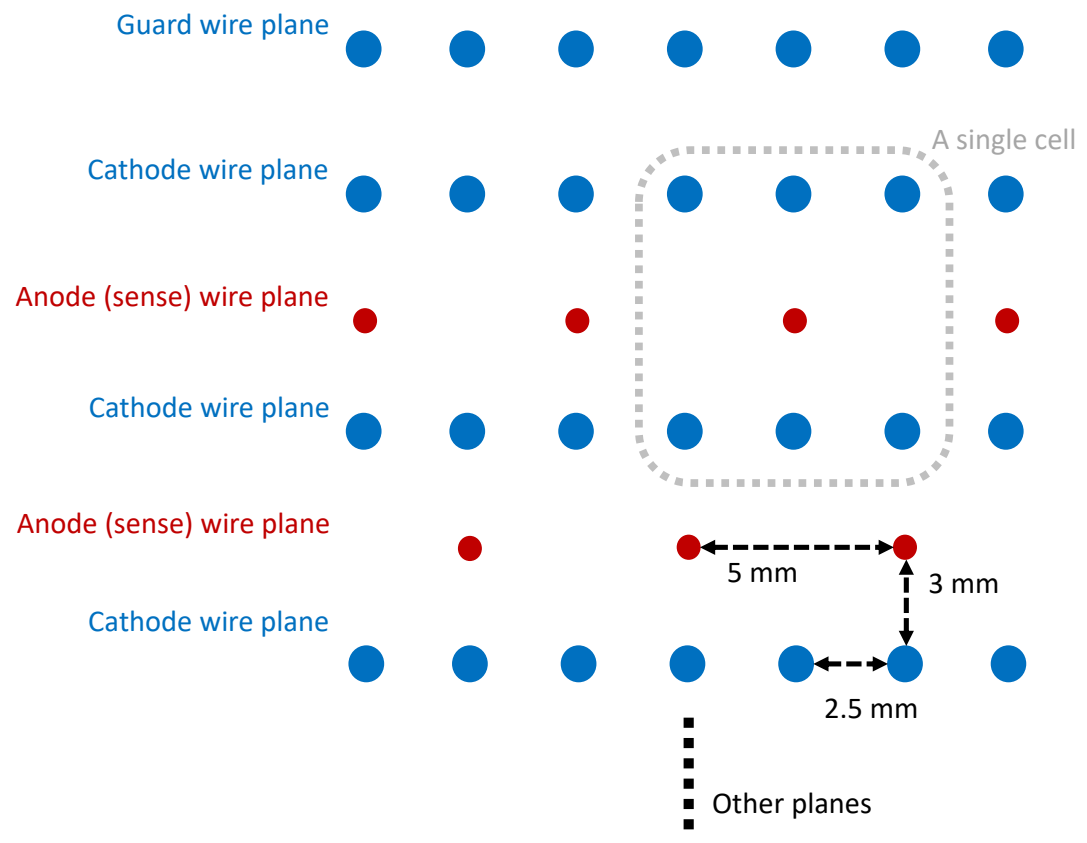

Figure A.3: Cell structure of the new Station-1 chamber.
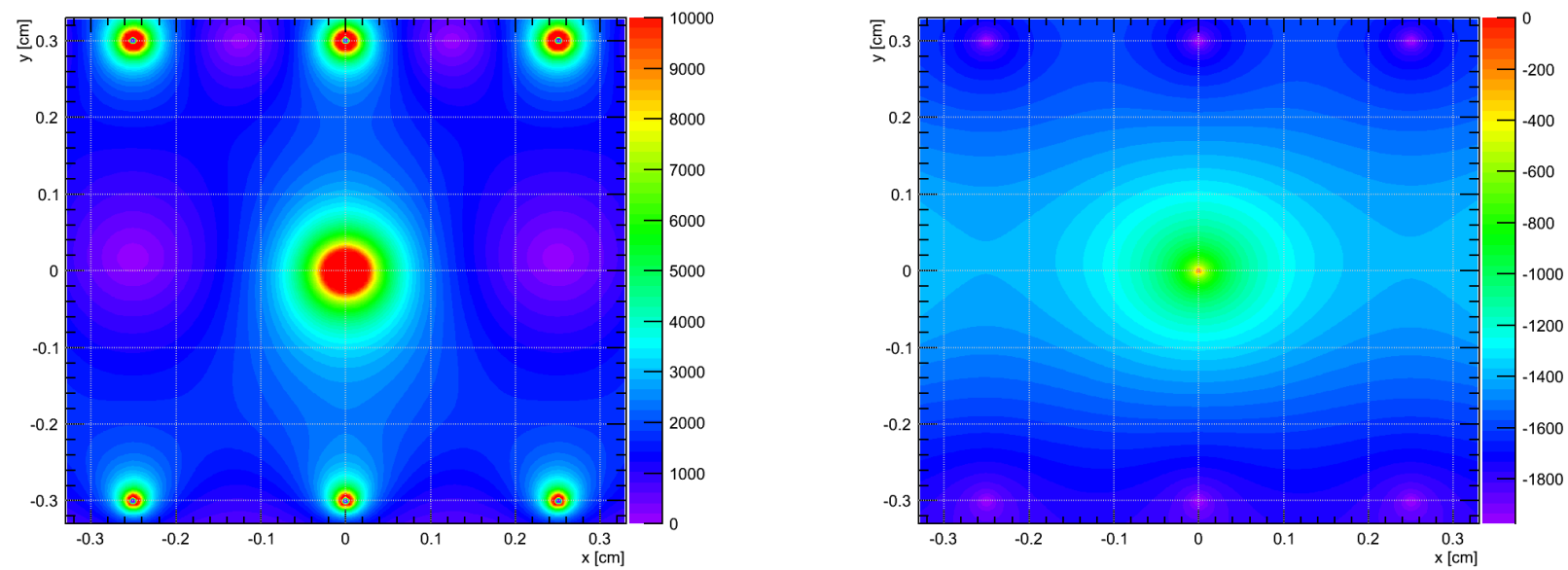

Figure A.4: The single-cell electric field (left) and potential (right) simulated by Garfield.

on G10 frames, on which the designed electric circuits are printed. The wires are soldered on the G10 frame, and each frame can thus be used as a chamber plane. For D1.2, a different approach is adopted by using metal pins and feedthroughs to hold the wires in place. In general, the wires 


\begin{tabular}{l|cc}
\hline Specifications & Cathode \& Guard & Anode \\
\hline Radius $(\mu \mathrm{m})$ & 38.1 & 10.15 \\
Linear Density $(\mathrm{kg} / \mathrm{m})$ & $3.825 \times 10^{-5}$ & $6.15 \times 10^{-6}$ \\
Length $(\mathrm{m})$ & 1.873 & $\mathrm{X}: 1.873, \mathrm{U} / \mathrm{V}: 1.931$ \\
Wire spacing $(\mathrm{mm})$ & 5 & 2.5 \\
\hline
\end{tabular}

Table A.1: Wire specifications of the new Station-1 chamber. The anode wires come in two lengths depending on the corresponding view.

can be more accurately positioned by this approach. However, once there is a broken wire, the wire repair process can be fairly challenging due to the fact that different planes cannot be simply separated. The general concept of this pin-and-feedthrough method is illustrated in Fig. A.5. In D1.2, the feedthrough is made by Delrin and has a 0.007-inch small opening on one end to confine the positon of the threaded wire. The gold-plated copper pin is tapered so that it can be pressfitted into the other end of the feedthrough. As in wire stringing, a wire was threaded through a feedthrough first, then the feedthrough was set on a hole precisely drilled on a end plate of the wire frame. Appropriate weight is subsequently applied on the wire to have it tensioned. A drop of super glue (LOCTITE 420 industrial instant adhesive) was put on the tip the pin and the pin was pushed into the feedthrough. The reason of using the super glue, not solder, to hold the wire was due to the heat damage on the feedthrough that can be caused by soldering. After each wire was strung, tests were made on every wire to ensure the pins have good electrical connection with the wires, not affected by the super glue. One thing to be noticed is that the pins are customized in two different lengths in order to make room for the signal readout and high-voltage application on the long pins.

During operation, high voltage is applied to the cathode wires so that a large electric field can be generated around the anode wires. If the tension of the wires is insufficient, the electrostatic force between wires can cause displacement or even damage to the wires. As discussed in [124], the minimum wire tension required, $T_{C}$, can be derived by calculating the equilibrium state for a wire 

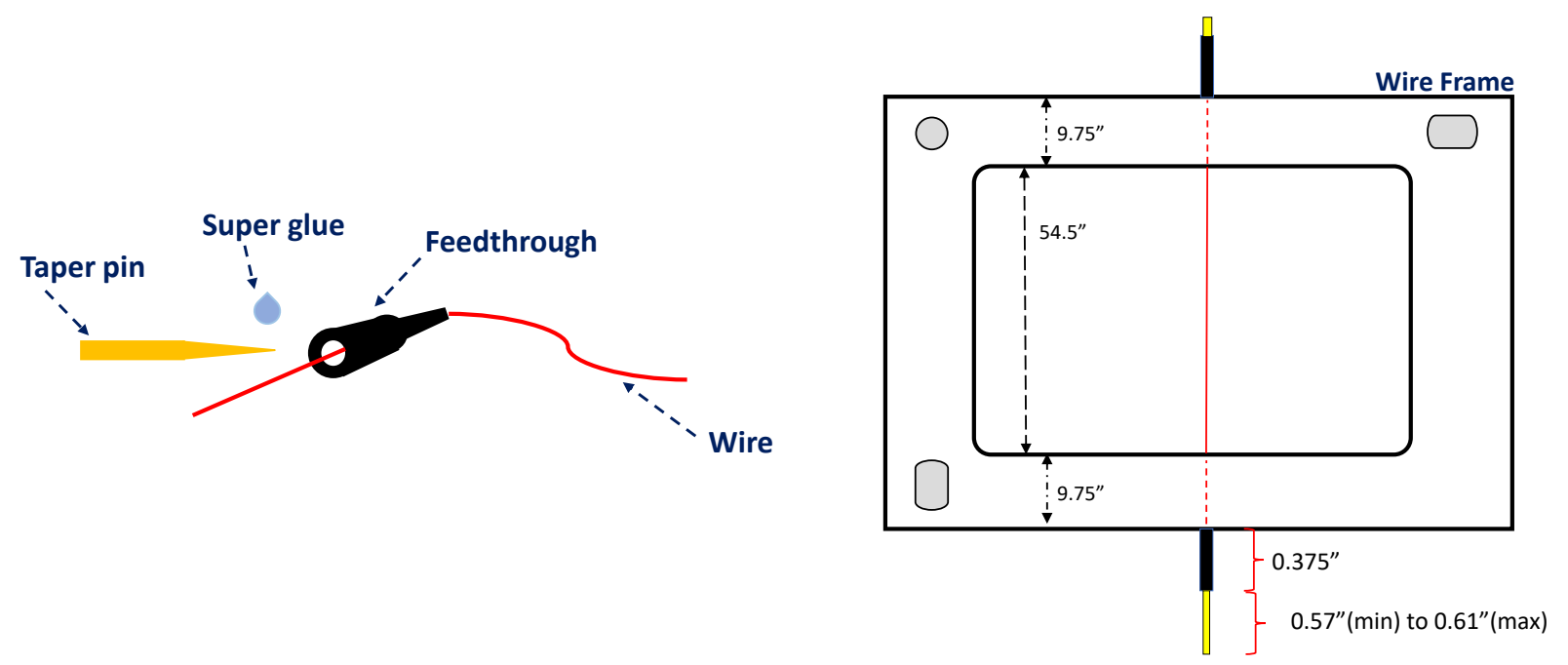

Figure A.5: Illustration of pin-and-feedthrough wire stringing method. The plot one the right shows how a wire is attached on the wire frame. The grey spots on the wire frame are slots for the insertion of brass pins that are used for mounting the wire frame in the gas box. Notice that the pin and feedthrough shown on the wire frame are not drawn to scale. There are two different lengths of pins and only the long ones are used for high-voltage or readout connections.

under the effects of electrostatic force and tension. The expression of $T_{C}$ is

$$
T_{C}=\frac{1}{4 \pi \epsilon_{0}}\left(\frac{C V_{0} L}{s}\right)
$$

where $\epsilon_{0}$ is the permittivity of free space, $C$ is the single-wire capacitance per unit length, $L$ is the wire length, and $s$ the wire spacing. The capacitance $C$ is given by

$$
C=\frac{2 \pi \epsilon_{0}}{(\pi l / s)-\ln (2 \pi a / s)}
$$

in which $l$ is the gap between planes, and $a$ is the wire radius. For D1.2, by using the numbers listed in Tab. A.1 and the 3-mm gap, then with an assumed working voltage of $2250 \mathrm{~V}$ one can derive the $T_{C}$ values as approximately $51.7 \mathrm{gf}$ for the straight anode wires, $54.9 \mathrm{gf}$ for the tilted ones, and $215.7 \mathrm{gf}$ for the cathode wires. In practice, since pressing the pin into the feedthrough during wire stringing can reduce the wire tension, the weights actually applied on wires were slightly greater than the values listed above, as $72 \mathrm{gf}$ for anode and $268 \mathrm{gf}$ for cathode wires. 
Evidently, with the large number of wires strung on the wire frame, the deformation of the frame brought by the wire tension needed to be considered since it can cause noticeable tension loss on the wires that are strung first. This issue was compensated by the "pre-tensioning" of the wire frame. Before wire stringing, the wire frame was ensembled first, set up vertically, and the top of it was then loaded by lead bricks that had a total weight similar to the sum of the wire tension. The resulting deformation was measured, and the turnbuckles were then employed on both sides of the wire frame to bend the wire frame to approximately the same degree. After all the wires were strung, the turnbuckles were removed.

After the wires are strung, the tension of each wire was examined by its resonant frequency. As we know, the resonant frequency $F$ of a wire of tension $T$ is

$$
F=\frac{n}{2 L} \sqrt{\frac{T}{\lambda}}, n=1,2,3, \ldots
$$

where $L$ is the length and $\lambda$ the linear density of the wire. In the tension test, the resonance of a wire was induced by the periodic Lorentz force produced with a magnet and a function generator. The magnet was set adjacent to the wire to be tested, and the wire was injected with the periodic current coming from the function generator. With magnetic field pointing vertically, the horizontal vibration of the wire could be directly observed by eye, and the frequency at which $n=1$ resonance occurred was determined as the corresponding frequency of the current. Referring to the linear densities given in the Tab. A.1 and the minimum tension discussed above, the resonant frequency was required to be greater than approximately $62 \mathrm{~Hz}$ for the cathode, and $77 \mathrm{~Hz}$ for both the straight and tilted anode wires.

\section{A.1.1 High-voltage Distribution and Readout}

The high voltage (HV) connection of D1.2 is instrumented at the top and the signal readout is at the bottom. A specific signal-readout card, as shown in Fig. A.6 was designed for SeaQuest by Sten Hansen. The connection to the pins was made by employing the low-insertion-force (LIF) connector produced by Hypertac Electrical/Electronic Manufacturing, and the part number is 

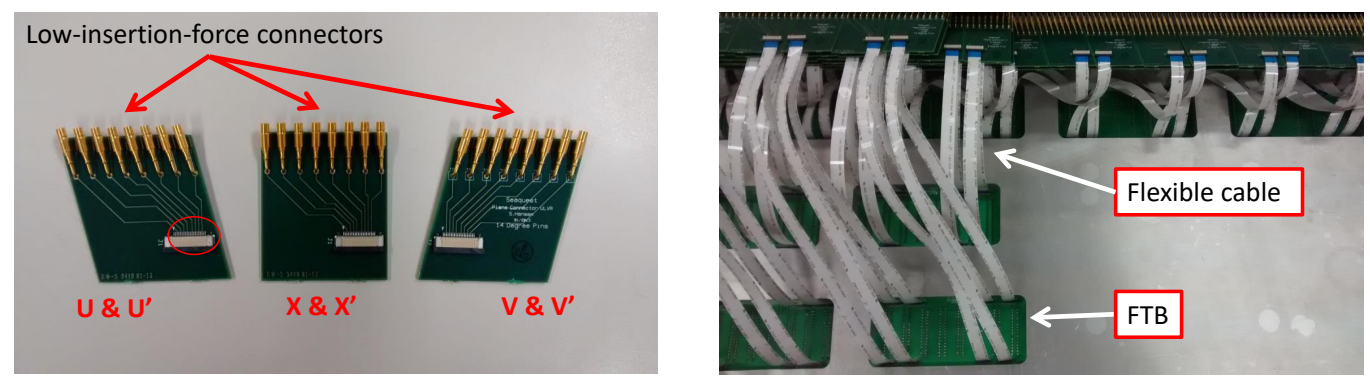

Figure A.6: Signal-readout card (left) and its connection to the Feedthrough-Boards (right) attached on the gas box. Different types of cards were used for planes of corresponding views.

YSK0102-049AH (0.102" inner opening diameter). The LIF connectors are soldered on to the printed circuit board which can accommodate eight channels. On the bottom of a card is the socket for a flexible cable connecting the card to the so-called Feedthrough-Board (FTB) attached on the gas box.

Since all the cathode planes are put at the same high voltage, only a HV NIM module was needed for the HV supply. A single HV cable delivers the HV from the module to all the cathode planes via the high-voltage distribution boards desgined by Sergey Loss. Each board has $8 \times 7=56$ pin connectors mounted and jumpers were utilized for the connection between different boards, as illustrated in Fig. A.7. The circuit inside the connector was specially designed so that the HV is distributed in a "zigzag" pattern among different planes, and every group of eight wires shares a current limiting resistor of $500 \mathrm{~K} \Omega$. With this design, no voltage difference can occur between adjacent cathodes at the edge from different boards if there is a sudden electric discharge between the cathodes and anodes (spark). In addition to the cathode planes, the two guard planes were also put at a voltage that is roughly half of the HV applied on the cathodes in order to better balance the electrostatic force between the outer planes. 

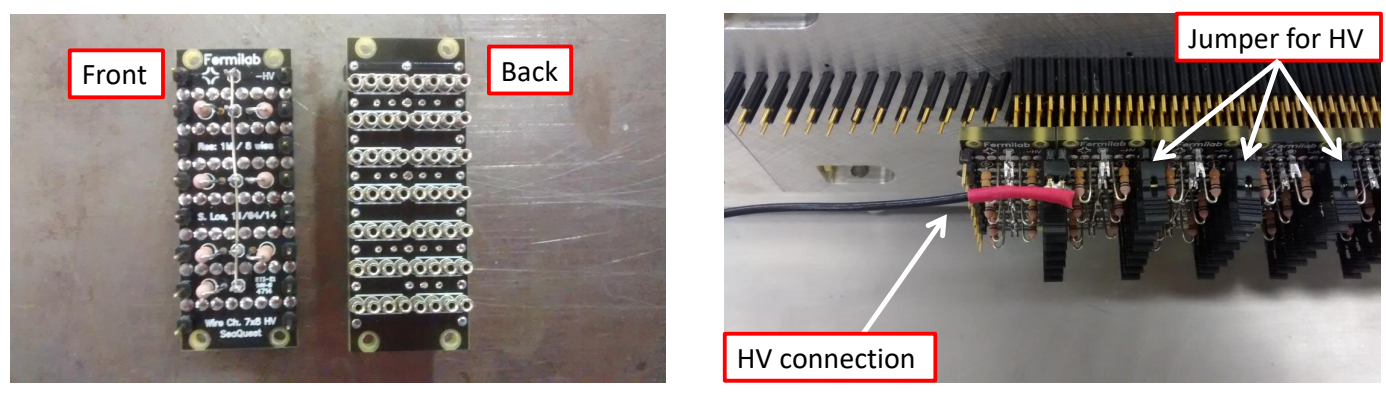

Figure A.7: The high-voltage distribution board (left) and its implementation (right).

\section{A.2 The Gas Box}

A picture of the gas box structure, without the lid of the box, is shown in Fig. A.8. The slots on the bottom are used for the Feedthrough-Boards and the opening on the top were made for panels of HV distribution. The window in the center has the dimension that matches the active area of the wire frame, and there is a window of the same size on the lid, too. Both the windows are covered by thick $(>45 \mu \mathrm{m})$ aluminized mylar foils to prevent the permeation of moisture from the outside. For the box lid, the FTBs, and the HV panels, various O-rings were used to keep the gas box airtight. The airtightness of D1.2 is well above the Fermilab requirement for flowing flammable gas into it.

The FTBs are printed circuit boards acting as the bridge between the flexible readout cables inside the gas box and the ASDQ cards mounted on them. The FTBs are designed by David Christian and are shown in Fig. A.9. Each board is connected to four cables from each of the two planes of the same view. Metal stripes on the rim of the FTB are used for the grounding purpose. The corresponding contacting surface on the gas box was painted by Alodine Pen first to convert the aluminum oxide to a layer of chromium/aluminum oxcide, which is more conductive to ensure the FTBs are well grounded.

On the gas box, there are three precise round grooves with holes in the center around the corners of the gas box. These grooves are snug-fit for the brass pins that are used to attach the wire 


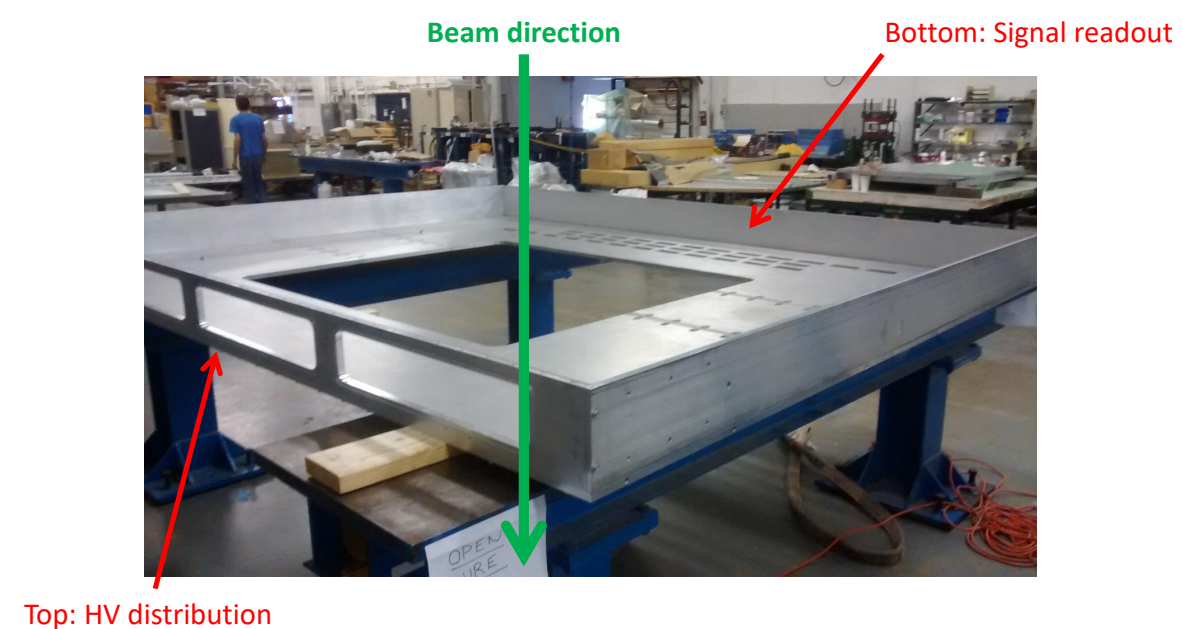

Figure A.8: The gas-box structure without the lid put on.

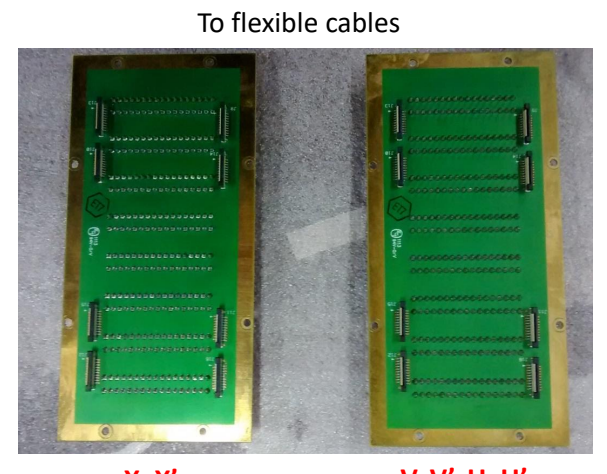

$x, x^{\prime}$

$V^{\prime}, V^{\prime}, U, U^{\prime}$

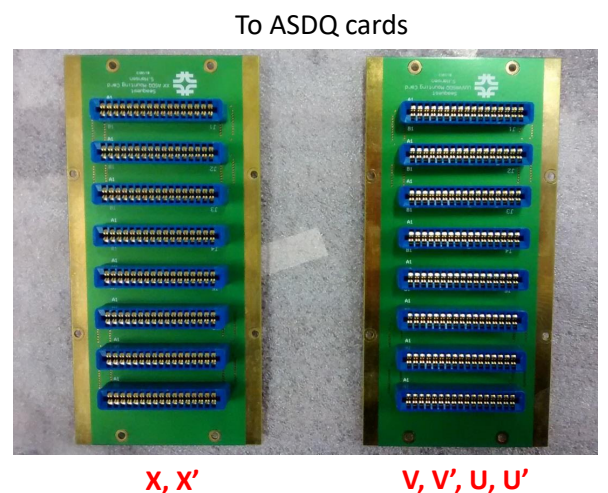

Figure A.9: The Feedthrough-Boards. The picture on the left shows the side that faces the inside of gas box, with connectors used for flexible cables. The right picture shows the sockets for ASDQ cards.

frame on the gas box. As illustrated in Fig. A.5. the three slots on the wire frame are made in a way that two of them have some adjustability for the brass pins in either the $\mathrm{X}$ or the $\mathrm{Y}$ direction. During installation of the wire frame, some RTV-11 silicone sealant was put on the surface of the groove for gas sealing. 


\section{Appendix B}

\section{Mass Spectrum Fitting with Random-mix Background}

The mass spectrum fitting given in this appendix demonstrates the ability to describe the measured mass distribution with the normalization of the random-mix events fixed by the $F_{b k g}$ method discussed in data analysis. The fittings are performed in the nominal $x_{F}$ bins. The Drell-Yan-event

yield extracted by fitting is not used in the $R_{p A}$ derivation, but only employed as a cross-check shown in Section 3.6.4.

In the following plots, the data points are represented by the black solid circles. The various fitting components and their sum are plotted in the color code identical to the one used in Section 3.6 .3 as:

- Drell-Yan: magenta line.

- $J / \psi$ and $\psi^{\prime}$ : red lines.

- Random-mix: black line.

- Sum of all componenets: blue line. 

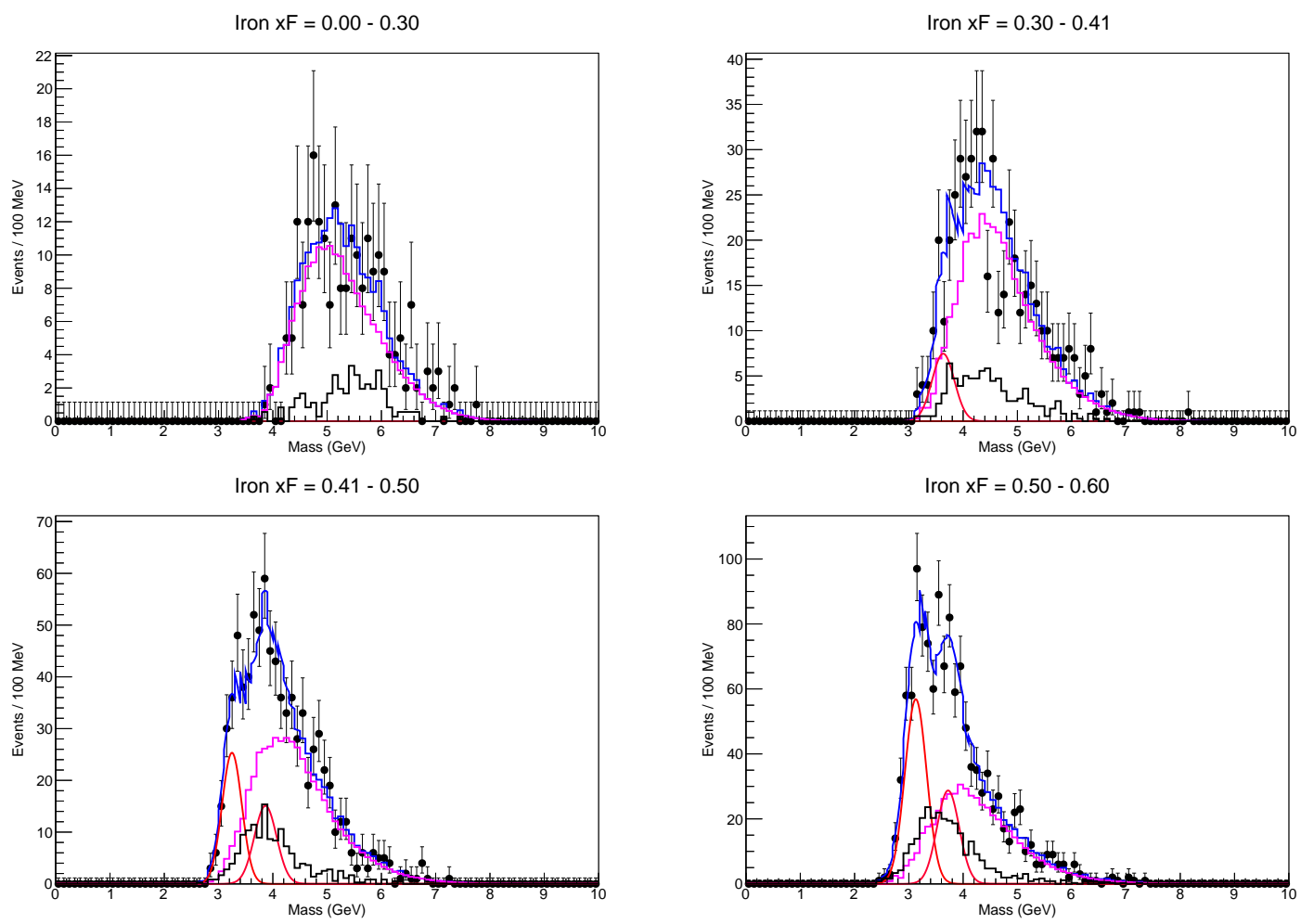

Iron $x F=0.60-0.67$

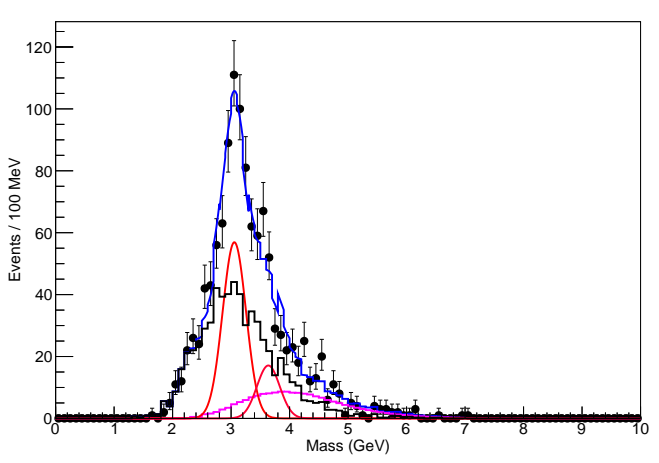

Figure B.1: Mass spectrum fitting in nominal $x_{F}$ bins of Run-II iron data. 

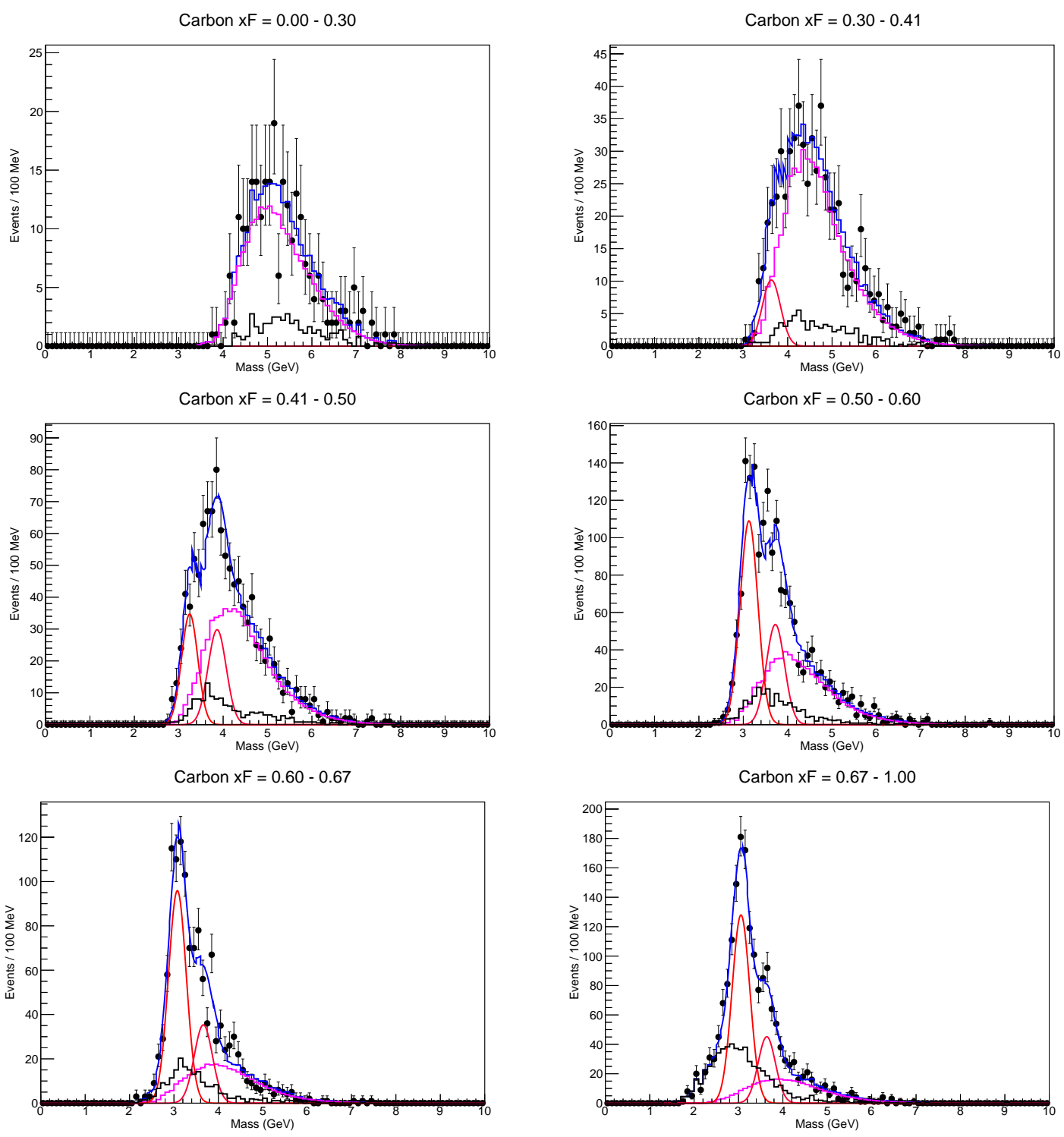

Figure B.2: Mass spectrum fitting in nominal $x_{F}$ bins of Run-II carbon data. 

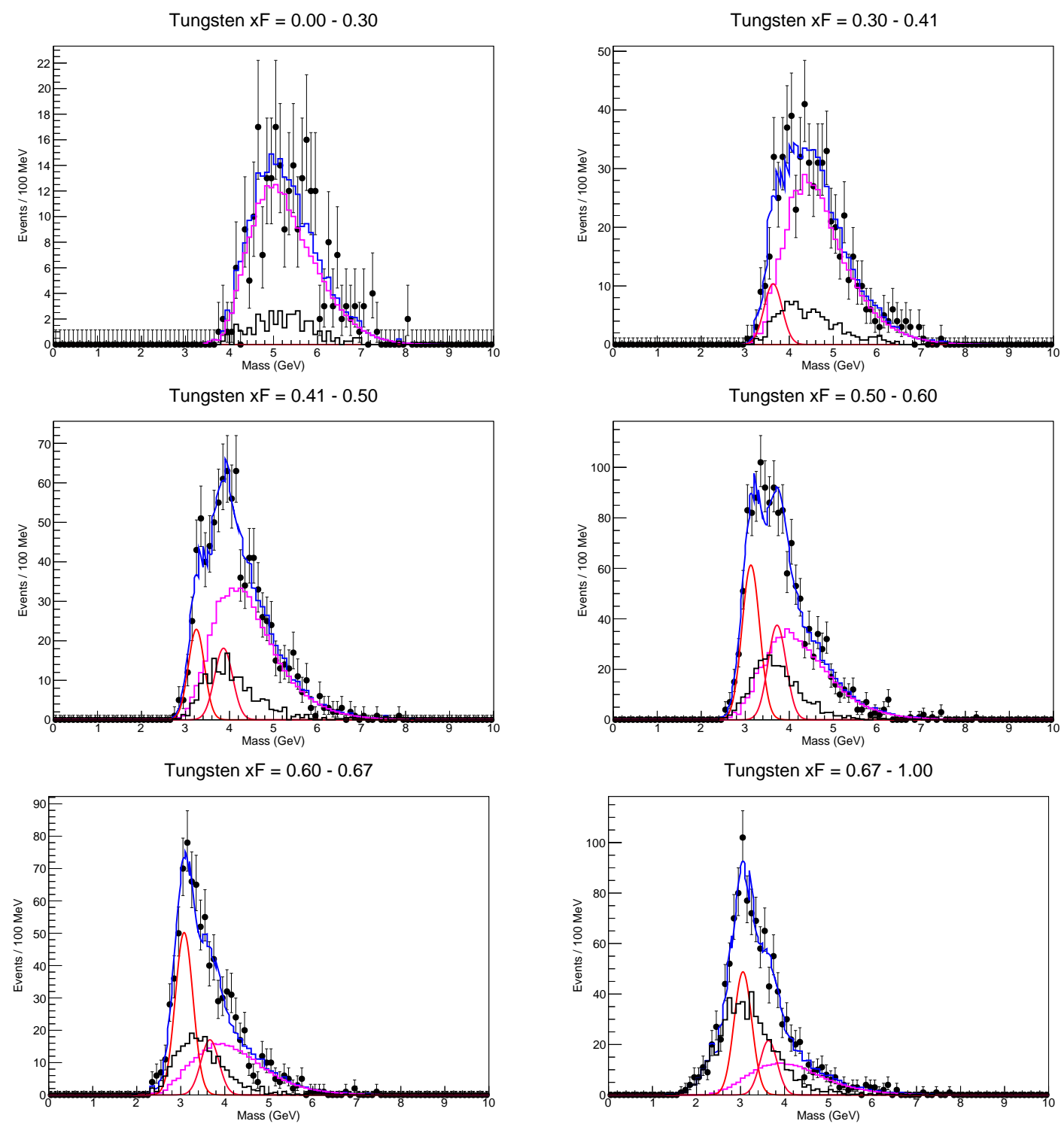

Figure B.3: Mass spectrum fitting in nominal $x_{F}$ bins of Run-II tungsten data. 

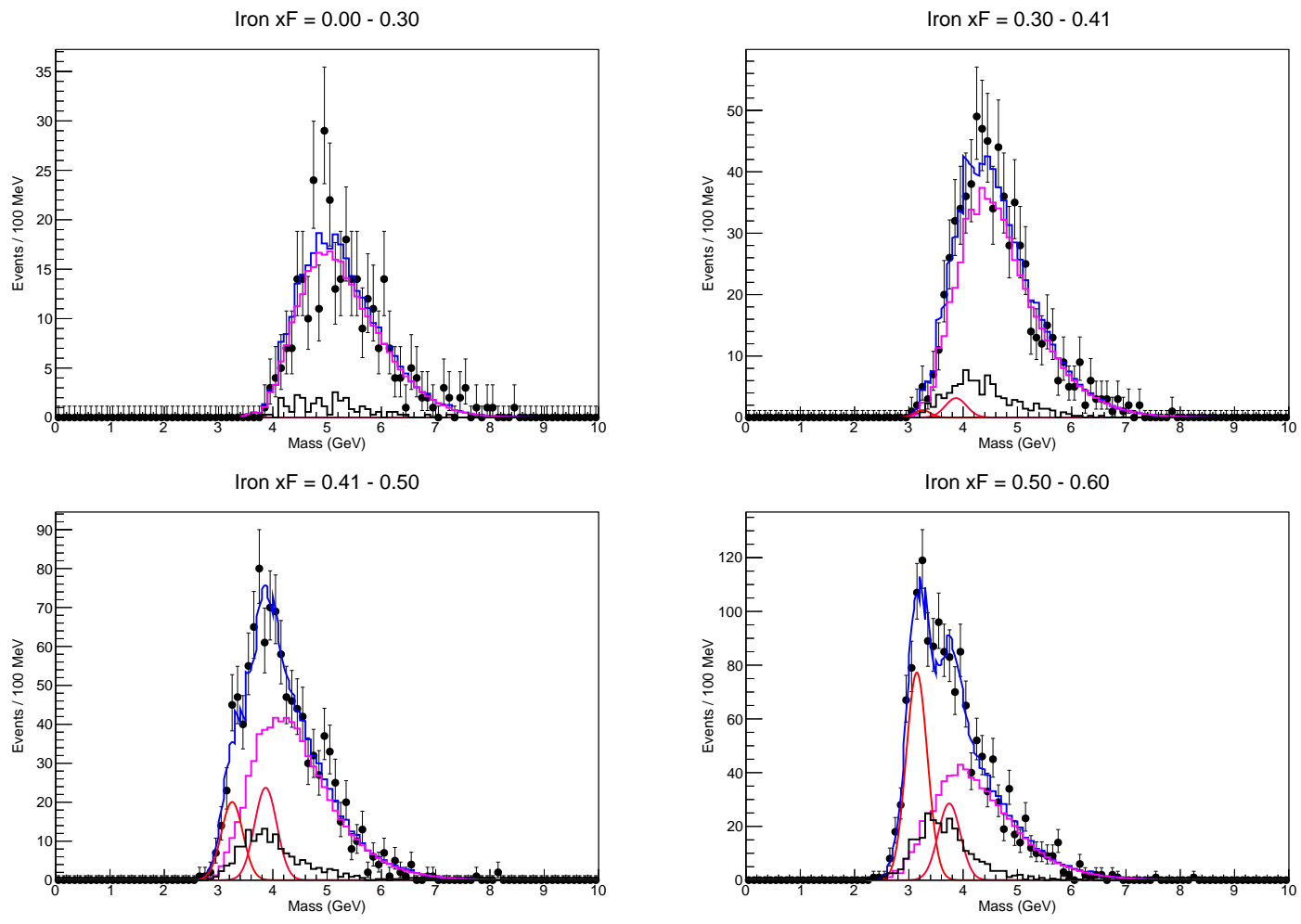

Iron $\mathrm{xF}=0.60-0.67$

Iron $\mathrm{xF}=0.67-1.00$
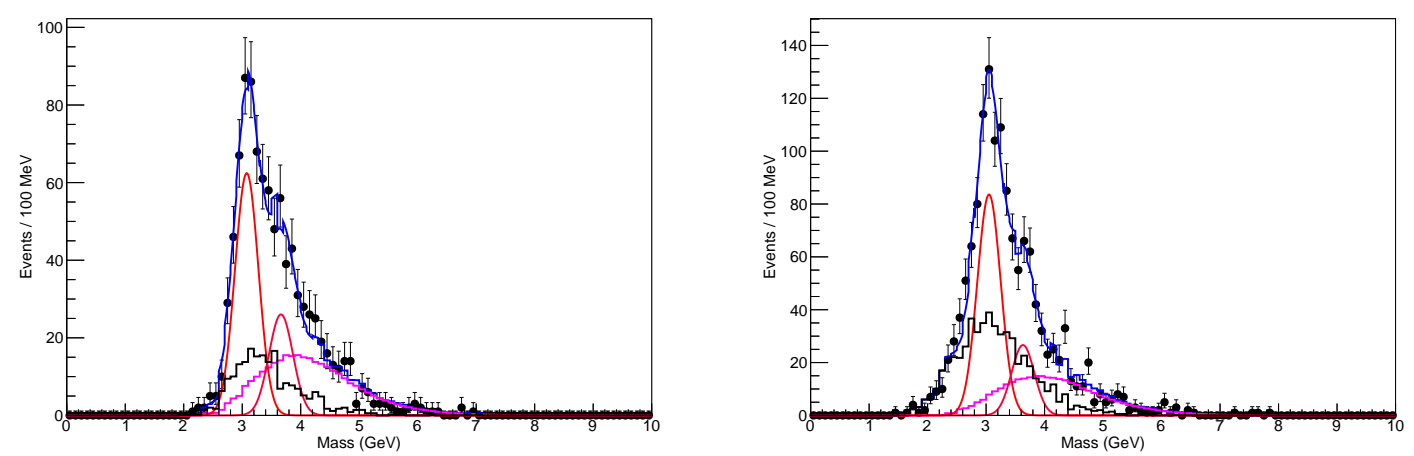

Figure B.4: Mass spectrum fitting in nominal $x_{F}$ bins of Roadset-62 iron data. 

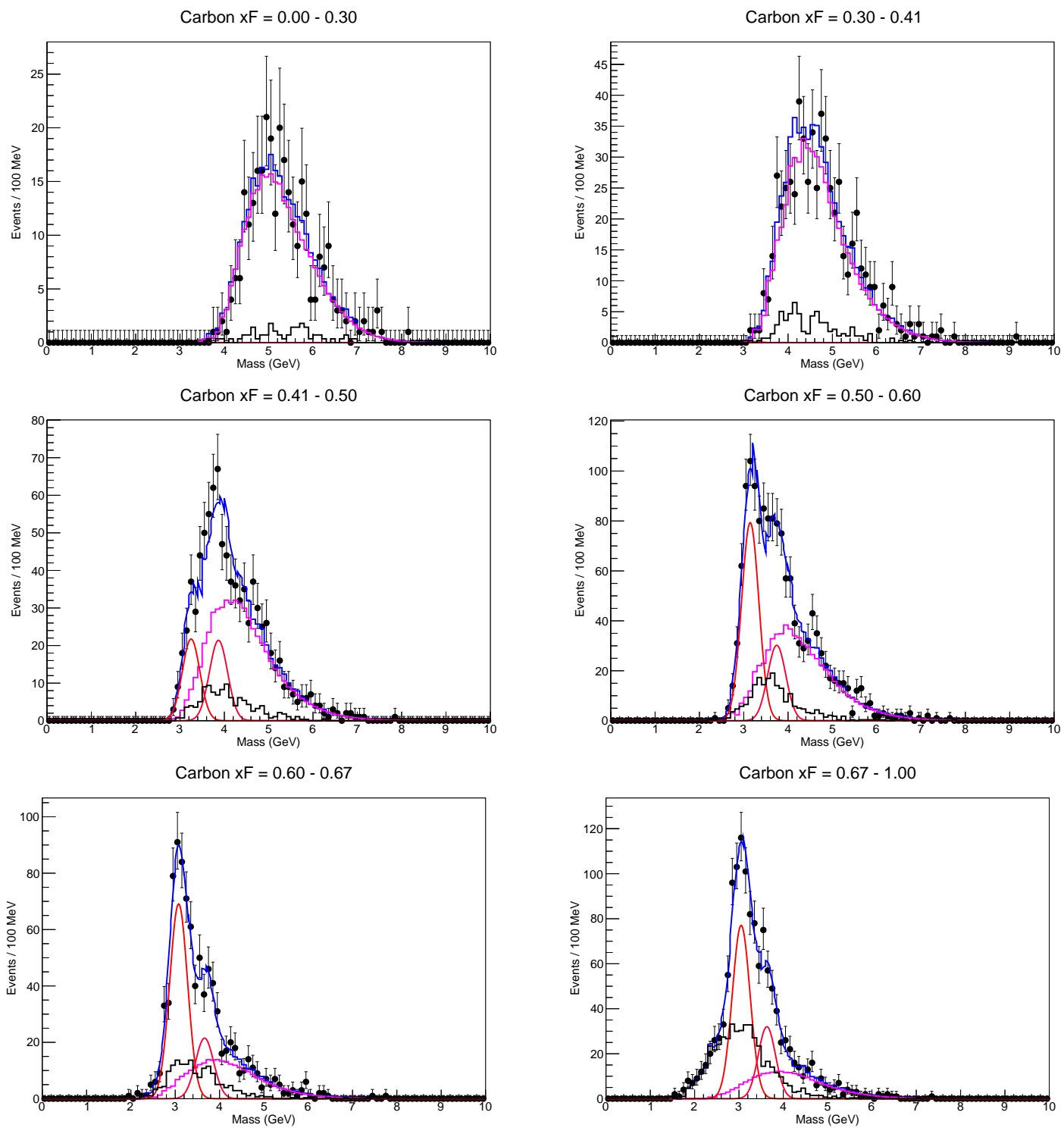

Figure B.5: Mass spectrum fitting in nominal $x_{F}$ bins of Roadset-62 carbon data. 

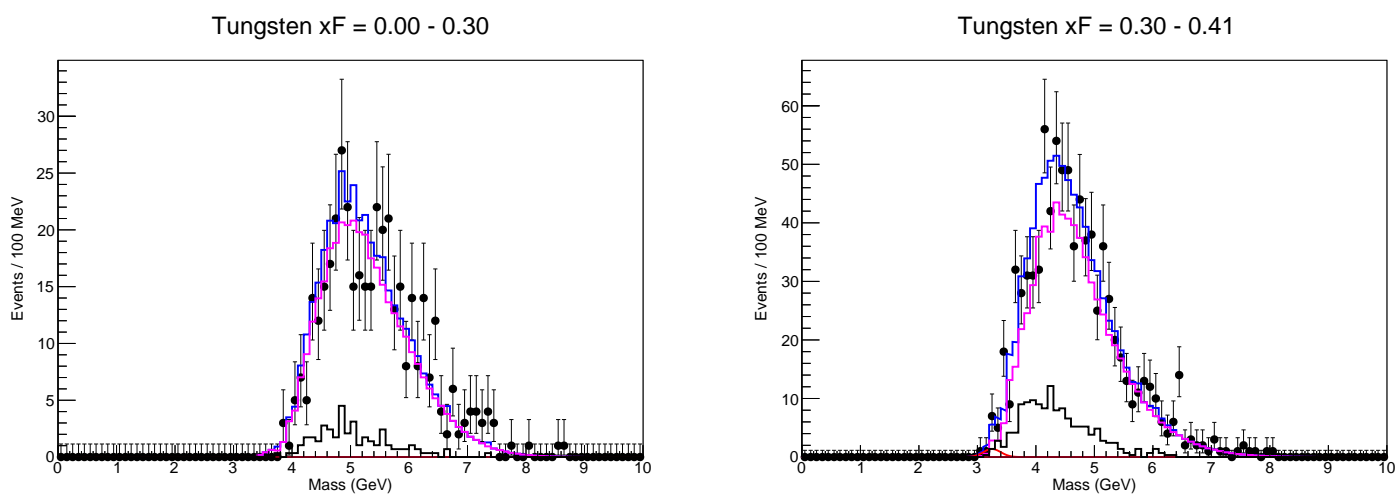

Tungsten $x F=0.41-0.50$
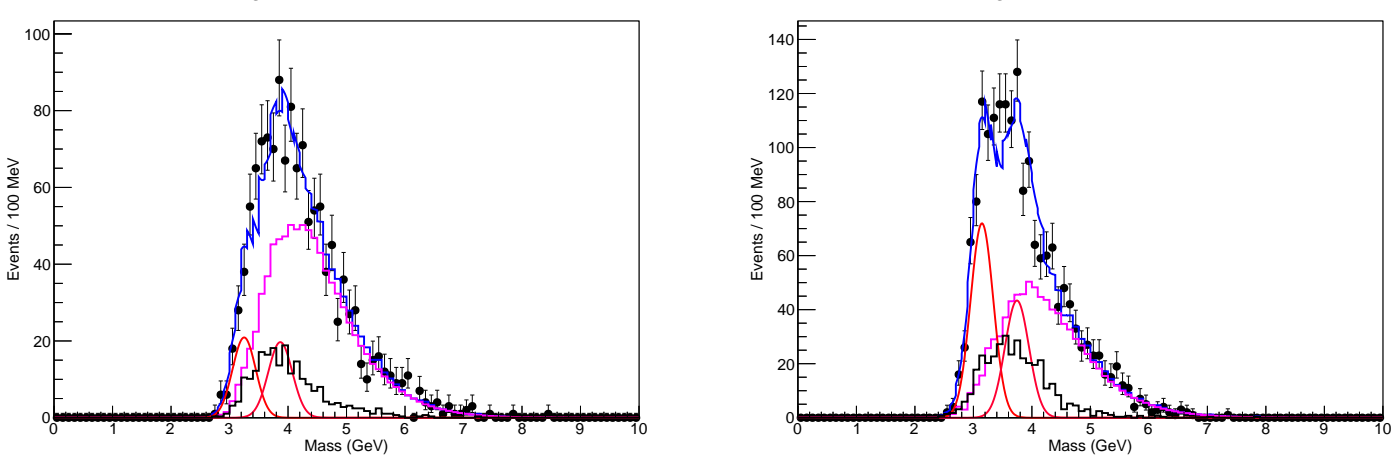

Tungsten $\mathrm{xF}=0.60-0.67$

Tungsten $x F=0.67-1.00$
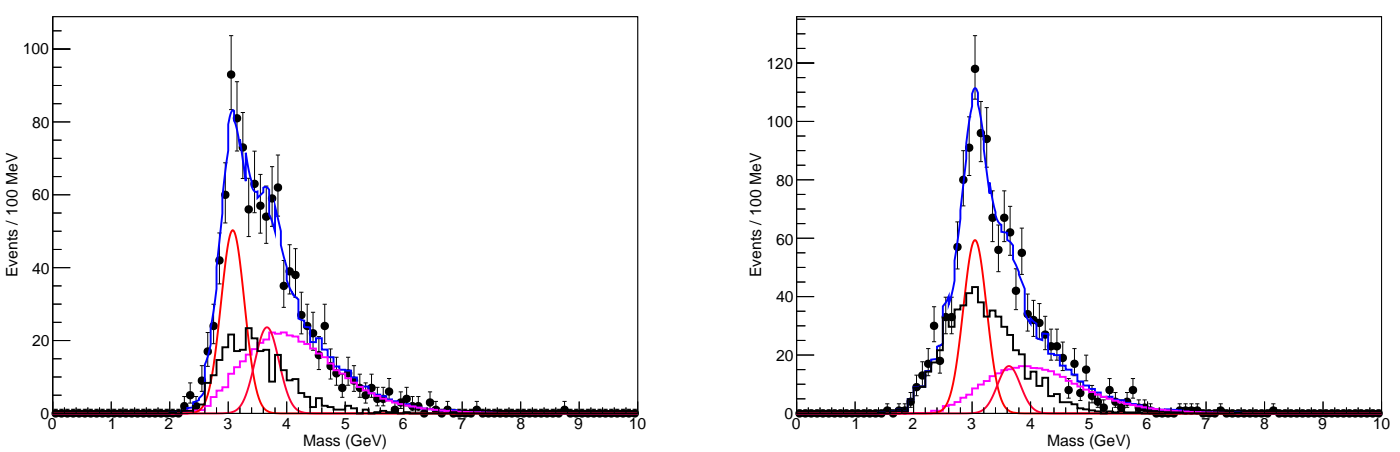

Figure B.6: Mass spectrum fitting in nominal $x_{F}$ bins of Roadset-62 tungsten data. 

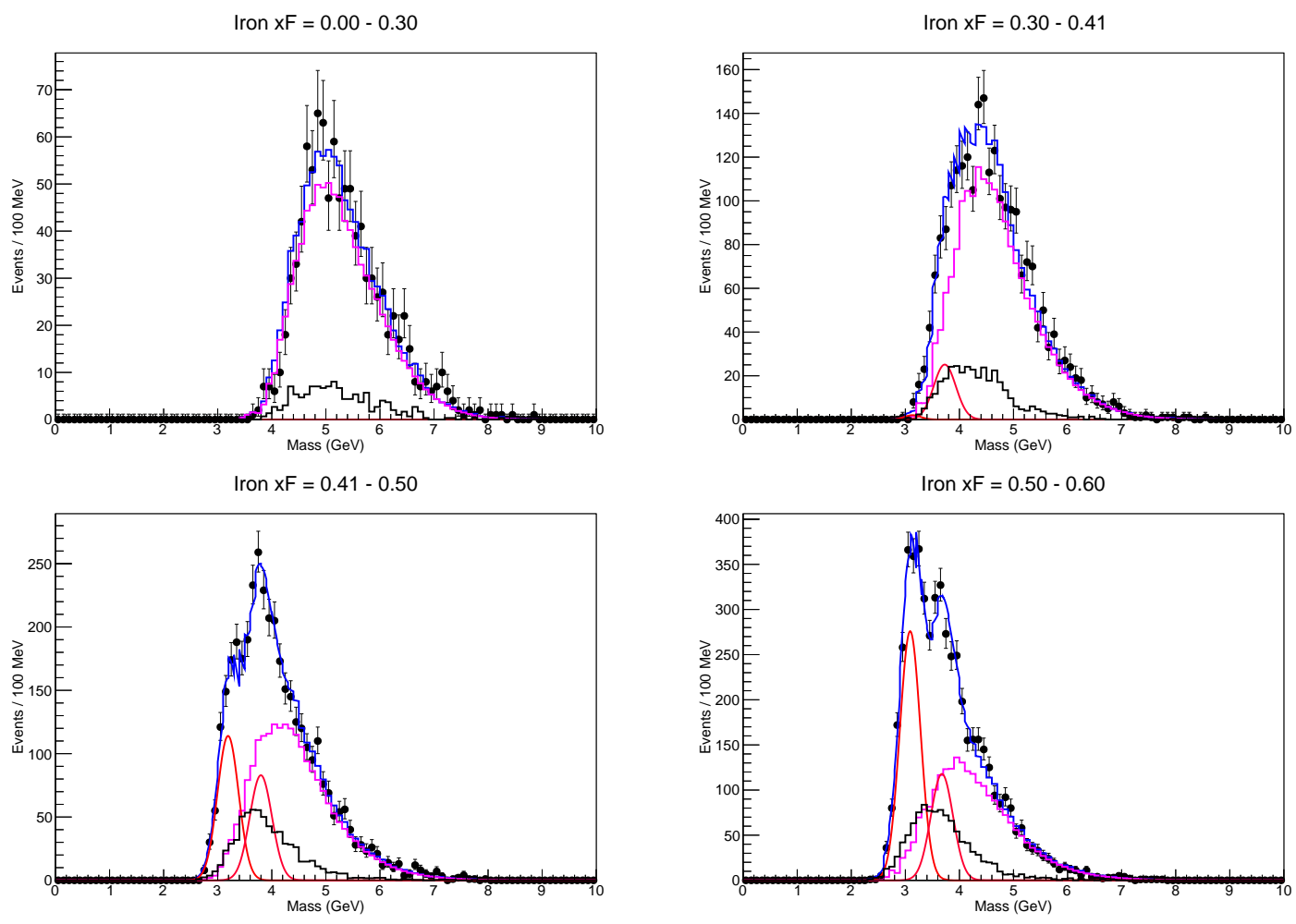

Iron $x F=0.60-0.67$

Iron $\mathrm{xF}=0.67-1.00$
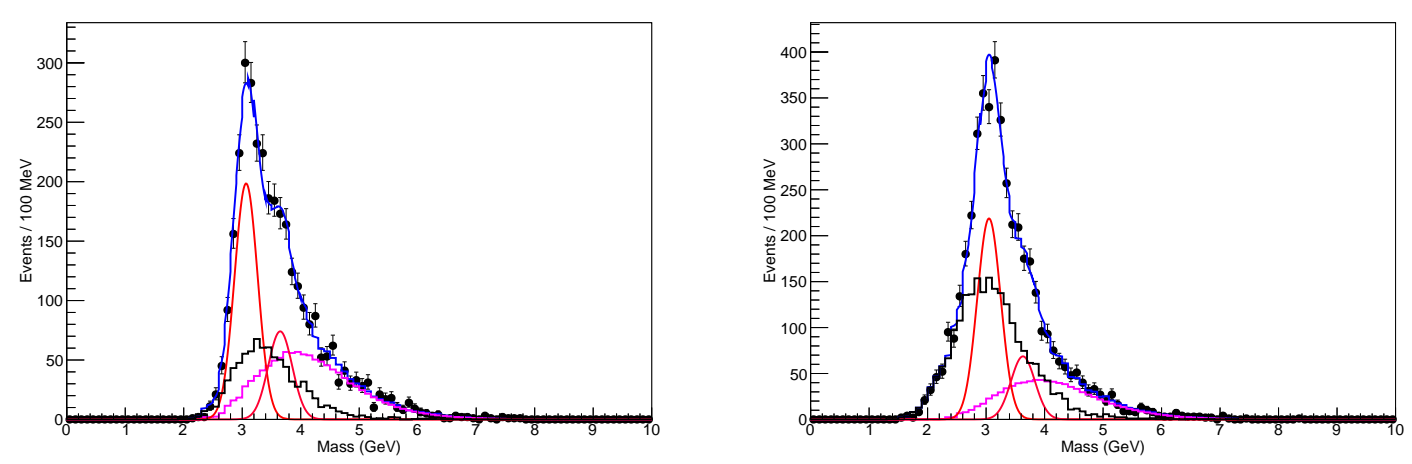

Figure B.7: Mass spectrum fitting in nominal $x_{F}$ bins of Roadset-67 iron data. 

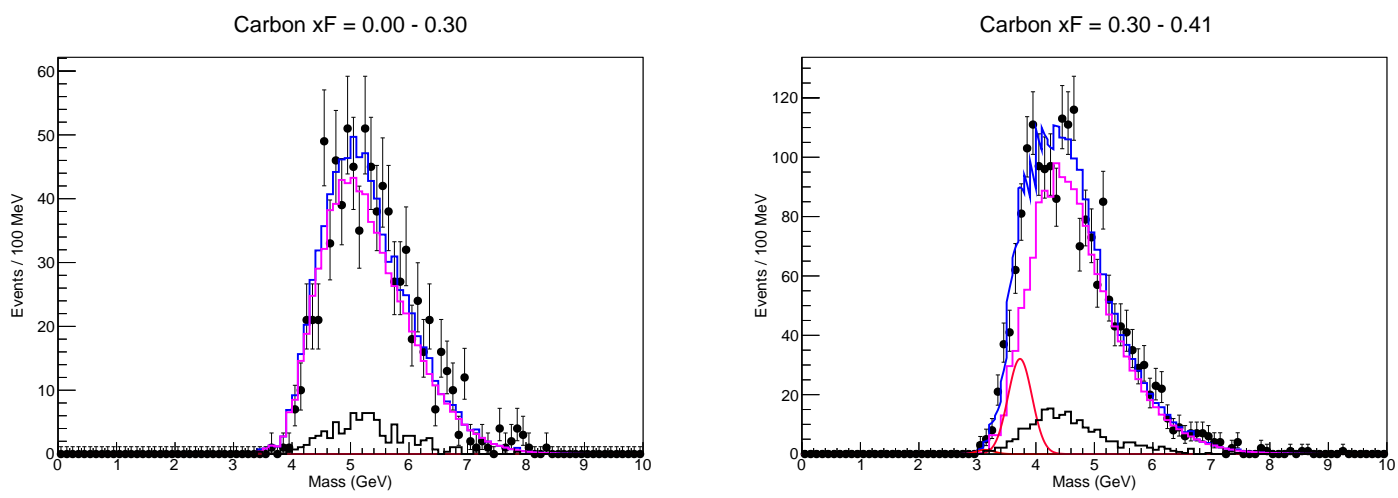

Carbon $\mathrm{xF}=0.41-0.50$
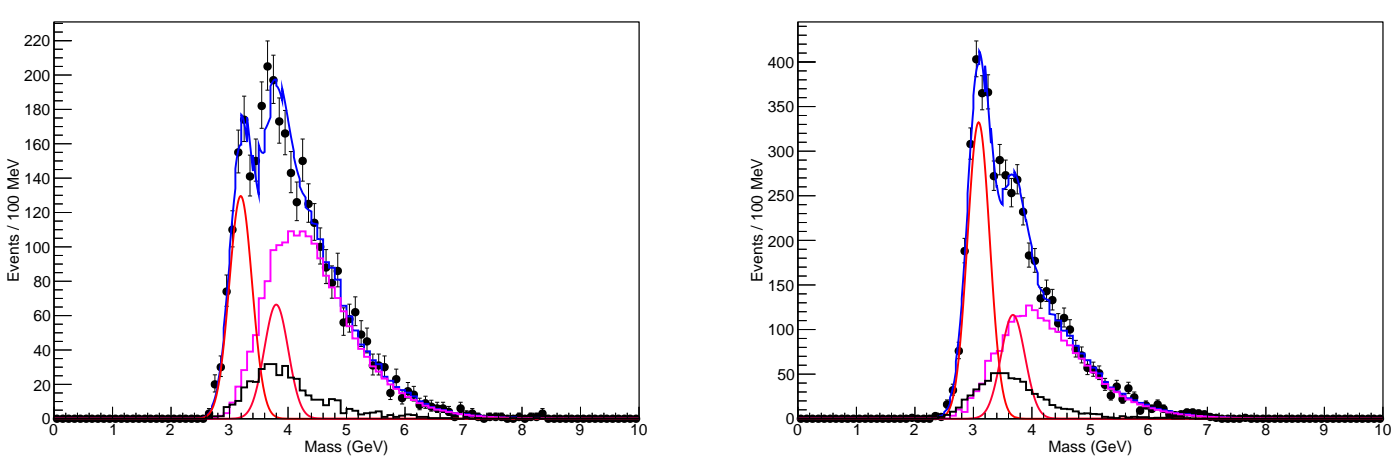

Carbon $x F=0.60-0.67$
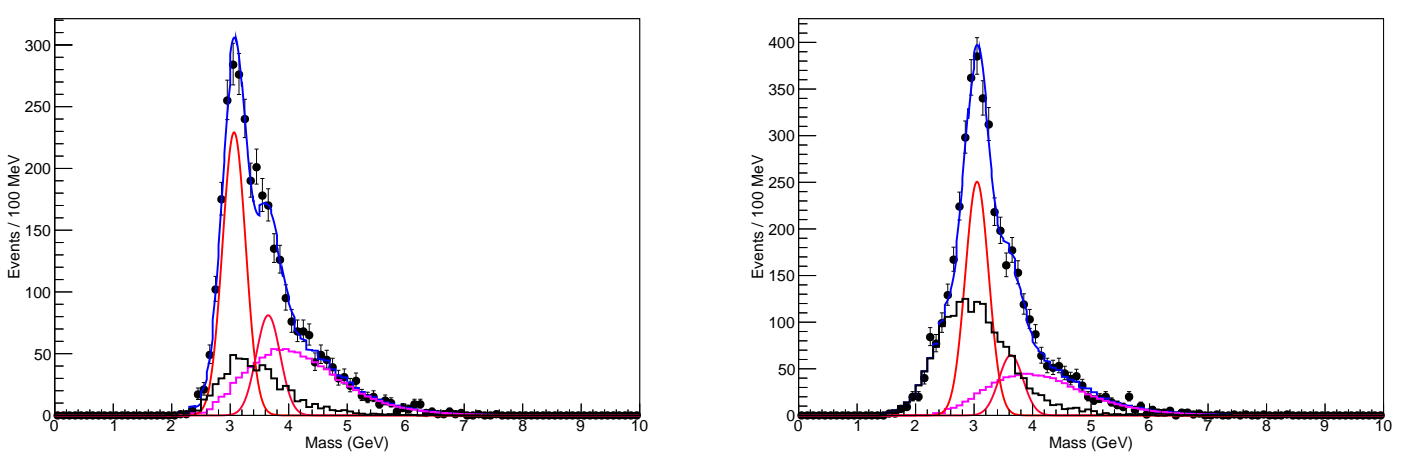

Figure B.8: Mass spectrum fitting in nominal $x_{F}$ bins of Roadset- 67 carbon data. 

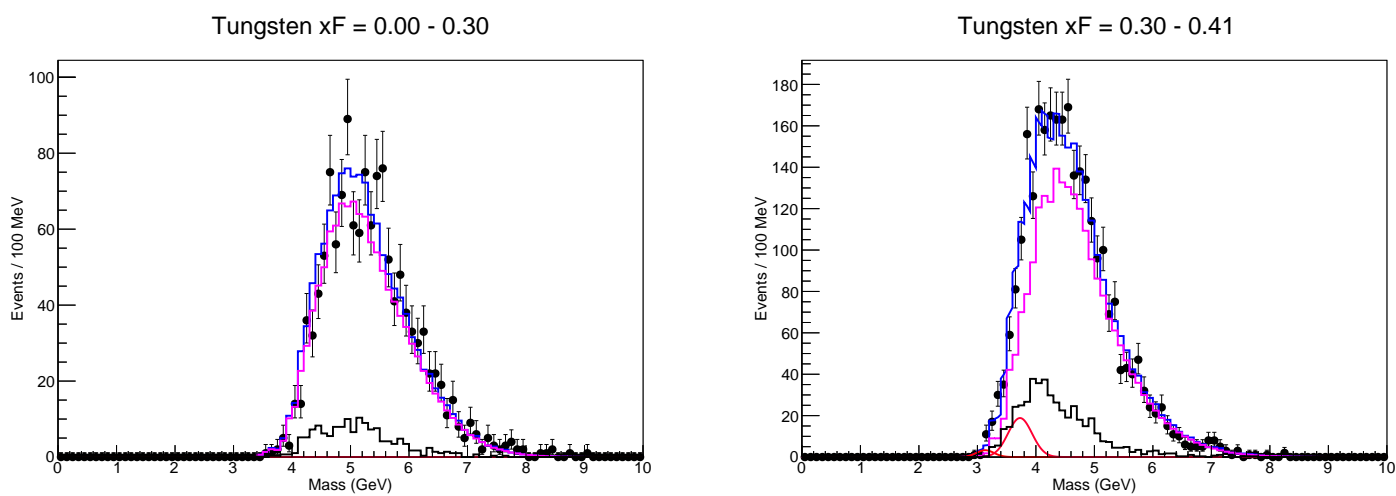

Tungsten $\mathrm{xF}=0.41-0.50$

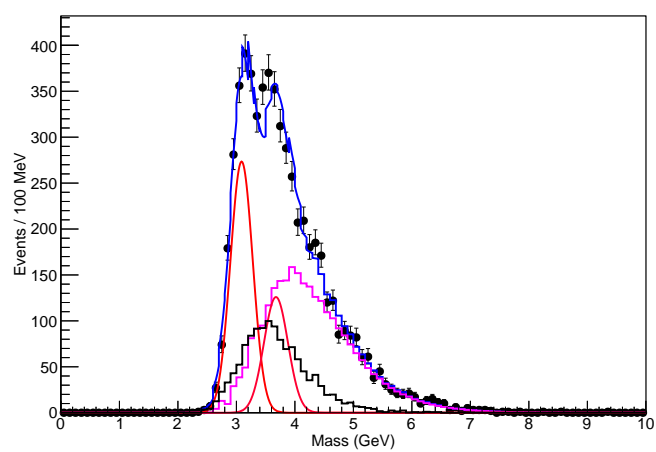

Tungsten $x F=0.67-1.00$
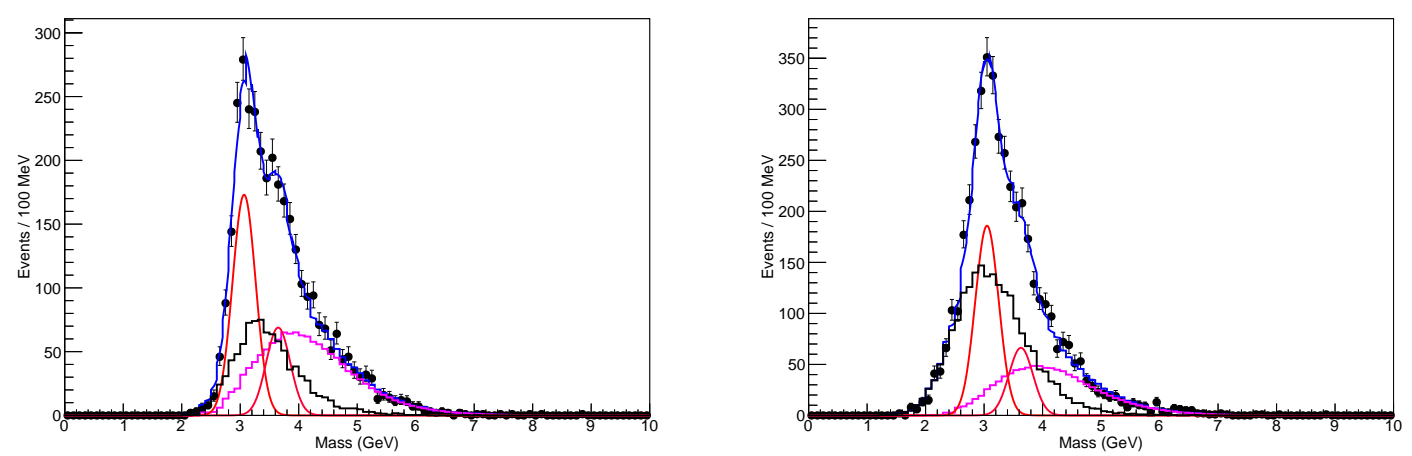

Figure B.9: Mass spectrum fitting in nominal $x_{F}$ bins of Roadset-67 tungsten data. 


\section{Appendix C \\ Supplementary Plots and Tables for $R_{p A}\left(x_{F}\right)$ with $x_{2}>0.15$}

With the $x_{2}>0.15$ requirement, the supplementary plots and tables related to the derivation of $R_{p A}\left(x_{F}\right)$ are given in this appendix. Since the analysis procedures are identical to the $R_{p A}$ evaluation without the $x_{2}$ cut, the detailed explanation of the materials presented, which can be found in the chapter of data analysis, is not given.

\section{C.1 Drell-Yan Yield Extraction and Average of Kinematics}

\begin{tabular}{cccccc}
\hline Target & $x_{F}$ bin & $N^{\text {raw }}$ & $N^{\text {bkg }}$ & $N^{\text {bkg }} / N^{\text {raw }}(\%)$ & $Y^{D Y}$ \\
\hline Fe & $0.00<x_{F} \leq 0.30$ & 220 & 37 & 17 & 183 \\
& $0.30<x_{F} \leq 0.41$ & 312 & 52 & 17 & 260 \\
& $0.41<x_{F} \leq 0.50$ & 292 & 37 & 13 & 255 \\
& $0.50<x_{F} \leq 0.60$ & 193 & 30 & 16 & 163 \\
& $0.60<x_{F} \leq 0.67$ & 55 & 10 & 18 & 45 \\
& $0.67<x_{F} \leq 1.00$ & 28 & 9 & 34 & 19 \\
\hline $\mathrm{C}$ & $0.00<x_{F} \leq 0.30$ & 240 & 41 & 17 & 199 \\
& $0.30<x_{F} \leq 0.41$ & 412 & 51 & 12 & 361 \\
& $0.41<x_{F} \leq 0.50$ & 374 & 46 & 12 & 328 \\
& $0.50<x_{F} \leq 0.60$ & 245 & 32 & 13 & 213 \\
& $0.60<x_{F} \leq 0.67$ & 68 & 14 & 21 & 54 \\
& $0.67<x_{F} \leq 1.00$ & 47 & 11 & 24 & 36 \\
\hline $\mathrm{W}$ & $0.00<x_{F} \leq 0.30$ & 246 & 35 & 14 & 211 \\
& $0.30<x_{F} \leq 0.41$ & 403 & 62 & 15 & 341 \\
& $0.41<x_{F} \leq 0.50$ & 349 & 55 & 16 & 294 \\
& $0.50<x_{F} \leq 0.60$ & 211 & 31 & 14 & 180 \\
& $0.60<x_{F} \leq 0.67$ & 64 & 8 & 12 & 56 \\
& $0.67<x_{F} \leq 1.00$ & 50 & 10 & 20 & 40 \\
\hline
\end{tabular}

Table C.1: Summary of background subtraction of Run-II data. 


\begin{tabular}{cccccc}
\hline Target & $x_{F}$ bin & $N^{\text {raw }}$ & $N^{\text {bkg }}$ & $N^{\text {bkg }} / N^{\text {raw }}(\%)$ & $Y^{D Y}$ \\
\hline Fe & $0.00<x_{F} \leq 0.30$ & 308 & 24 & 8 & 284 \\
& $0.30<x_{F} \leq 0.41$ & 499 & 48 & 10 & 451 \\
& $0.41<x_{F} \leq 0.50$ & 425 & 43 & 10 & 382 \\
& $0.50<x_{F} \leq 0.60$ & 234 & 23 & 10 & 211 \\
& $0.60<x_{F} \leq 0.67$ & 55 & 9 & 16 & 46 \\
& $0.67<x_{F} \leq 1.00$ & 50 & 6 & 12 & 44 \\
\hline $\mathrm{C}$ & $0.00<x_{F} \leq 0.30$ & 288 & 20 & 7 & 268 \\
& $0.30<x_{F} \leq 0.41$ & 444 & 39 & 9 & 405 \\
& $0.41<x_{F} \leq 0.50$ & 323 & 38 & 12 & 285 \\
& $0.50<x_{F} \leq 0.60$ & 240 & 23 & 10 & 217 \\
& $0.60<x_{F} \leq 0.67$ & 57 & 7 & 13 & 50 \\
& $0.67<x_{F} \leq 1.00$ & 40 & 4 & 11 & 36 \\
\hline $\mathrm{W}$ & $0.00<x_{F} \leq 0.30$ & 387 & 36 & 9 & 351 \\
& $0.30<x_{F} \leq 0.41$ & 594 & 75 & 13 & 519 \\
& $0.41<x_{F} \leq 0.50$ & 511 & 55 & 11 & 456 \\
& $0.50<x_{F} \leq 0.60$ & 300 & 32 & 11 & 268 \\
& $0.60<x_{F} \leq 0.67$ & 85 & 10 & 11 & 75 \\
& $0.67<x_{F} \leq 1.00$ & 45 & 6 & 12 & 39 \\
\hline
\end{tabular}

Table C.2: Summary of background subtraction of Roadset 62 data.

\begin{tabular}{cccccc}
\hline Target & $x_{F}$ bin & $N^{\text {raw }}$ & $N^{\text {bkg }}$ & $N^{\text {bkg }} / N^{\text {raw }}(\%)$ & $Y^{D Y}$ \\
\hline Fe & $0.00<x_{F} \leq 0.30$ & 969 & 116 & 12 & 853 \\
& $0.30<x_{F} \leq 0.41$ & 1578 & 191 & 12 & 1387 \\
& $0.41<x_{F} \leq 0.50$ & 1280 & 169 & 13 & 1111 \\
& $0.50<x_{F} \leq 0.60$ & 793 & 99 & 12 & 694 \\
& $0.60<x_{F} \leq 0.67$ & 268 & 32 & 12 & 236 \\
& $0.67<x_{F} \leq 1.00$ & 148 & 23 & 15 & 125 \\
\hline $\mathrm{C}$ & $0.00<x_{F} \leq 0.30$ & 823 & 82 & 10 & 741 \\
& $0.30<x_{F} \leq 0.41$ & 1308 & 144 & 11 & 1164 \\
& $0.41<x_{F} \leq 0.50$ & 1109 & 114 & 10 & 995 \\
& $0.50<x_{F} \leq 0.60$ & 756 & 78 & 10 & 678 \\
& $0.60<x_{F} \leq 0.67$ & 236 & 29 & 12 & 207 \\
& $0.67<x_{F} \leq 1.00$ & 152 & 26 & 17 & 126 \\
\hline $\mathrm{W}$ & $0.00<x_{F} \leq 0.30$ & 1280 & 133 & 10 & 1147 \\
& $0.30<x_{F} \leq 0.41$ & 1885 & 237 & 13 & 1648 \\
& $0.41<x_{F} \leq 0.50$ & 1573 & 191 & 12 & 1382 \\
& $0.50<x_{F} \leq 0.60$ & 942 & 114 & 12 & 828 \\
& $0.60<x_{F} \leq 0.67$ & 283 & 37 & 13 & 246 \\
& $0.67<x_{F} \leq 1.00$ & 161 & 22 & 14 & 139 \\
\hline
\end{tabular}

Table C.3: Summary of background subtraction of Roadset 67 data. 


\begin{tabular}{ccccccc}
\hline Target & $x_{F}$ bin & $\left\langle x_{1}\right\rangle$ & $\left\langle x_{2}\right\rangle$ & $\left\langle x_{F}\right\rangle$ & $\langle$ mass $\rangle$ & $\left\langle p_{T}\right\rangle$ \\
\hline Fe & $0.00<x_{F} \leq 0.30$ & 0.473 & 0.279 & 0.223 & 5.388 & 0.68 \\
& $0.30<x_{F} \leq 0.41$ & 0.535 & 0.221 & 0.359 & 5.080 & 0.74 \\
& $0.41<x_{F} \leq 0.50$ & 0.593 & 0.192 & 0.454 & 4.974 & 0.82 \\
& $0.50<x_{F} \leq 0.60$ & 0.658 & 0.188 & 0.539 & 5.237 & 0.61 \\
& $0.60<x_{F} \leq 0.67$ & 0.718 & 0.175 & 0.625 & 5.277 & 0.68 \\
& $0.67<x_{F} \leq 1.00$ & 0.804 & 0.185 & 0.736 & 5.847 & 0.22 \\
\hline $\mathrm{C}$ & $0.00<x_{F} \leq 0.30$ & 0.477 & 0.271 & 0.237 & 5.352 & 0.66 \\
& $0.30<x_{F} \leq 0.41$ & 0.535 & 0.220 & 0.359 & 5.077 & 0.73 \\
& $0.41<x_{F} \leq 0.50$ & 0.592 & 0.193 & 0.453 & 4.999 & 0.71 \\
& $0.50<x_{F} \leq 0.60$ & 0.663 & 0.191 & 0.543 & 5.255 & 0.77 \\
& $0.60<x_{F} \leq 0.67$ & 0.725 & 0.183 & 0.627 & 5.392 & 0.69 \\
& $0.67<x_{F} \leq 1.00$ & 0.789 & 0.178 & 0.715 & 5.591 & 0.57 \\
\hline $\mathrm{W}$ & $0.00<x_{F} \leq 0.30$ & 0.477 & 0.288 & 0.219 & 5.474 & 0.81 \\
& $0.30<x_{F} \leq 0.41$ & 0.532 & 0.215 & 0.359 & 4.994 & 0.77 \\
& $0.41<x_{F} \leq 0.50$ & 0.594 & 0.193 & 0.455 & 4.997 & 0.78 \\
& $0.50<x_{F} \leq 0.60$ & 0.664 & 0.189 & 0.546 & 5.227 & 0.77 \\
& $0.60<x_{F} \leq 0.67$ & 0.724 & 0.183 & 0.627 & 5.403 & 0.64 \\
& $0.67<x_{F} \leq 1.00$ & 0.794 & 0.189 & 0.716 & 5.734 & 0.67 \\
\hline
\end{tabular}

Table C.4: Average values of kinematic variables of Run-II data.

\begin{tabular}{ccccccc}
\hline Target & $x_{F}$ bin & $\left\langle x_{1}\right\rangle$ & $\left\langle x_{2}\right\rangle$ & $\left\langle x_{F}\right\rangle$ & $\langle$ mass $\rangle$ & $\left\langle p_{T}\right\rangle$ \\
\hline Fe & $0.00<x_{F} \leq 0.30$ & 0.472 & 0.283 & 0.218 & 5.391 & 0.83 \\
& $0.30<x_{F} \leq 0.41$ & 0.531 & 0.213 & 0.360 & 4.967 & 0.79 \\
& $0.41<x_{F} \leq 0.50$ & 0.592 & 0.192 & 0.453 & 4.967 & 0.78 \\
& $0.50<x_{F} \leq 0.60$ & 0.664 & 0.188 & 0.545 & 5.200 & 0.80 \\
& $0.60<x_{F} \leq 0.67$ & 0.730 & 0.184 & 0.633 & 5.418 & 0.75 \\
& $0.67<x_{F} \leq 1.00$ & 0.801 & 0.194 & 0.721 & 5.811 & 0.80 \\
\hline $\mathrm{C}$ & $0.00<x_{F} \leq 0.30$ & 0.473 & 0.278 & 0.225 & 5.357 & 0.79 \\
& $0.30<x_{F} \leq 0.41$ & 0.537 & 0.220 & 0.361 & 5.081 & 0.76 \\
& $0.41<x_{F} \leq 0.50$ & 0.595 & 0.195 & 0.455 & 5.031 & 0.76 \\
& $0.50<x_{F} \leq 0.60$ & 0.660 & 0.188 & 0.541 & 5.191 & 0.79 \\
& $0.60<x_{F} \leq 0.67$ & 0.732 & 0.191 & 0.632 & 5.538 & 0.70 \\
& $0.67<x_{F} \leq 1.00$ & 0.798 & 0.182 & 0.721 & 5.591 & 0.87 \\
\hline $\mathrm{W}$ & $0.00<x_{F} \leq 0.30$ & 0.481 & 0.290 & 0.222 & 5.514 & 0.82 \\
& $0.30<x_{F} \leq 0.41$ & 0.535 & 0.220 & 0.359 & 5.075 & 0.77 \\
& $0.41<x_{F} \leq 0.50$ & 0.592 & 0.195 & 0.451 & 5.010 & 0.77 \\
& $0.50<x_{F} \leq 0.60$ & 0.663 & 0.185 & 0.547 & 5.144 & 0.86 \\
& $0.60<x_{F} \leq 0.67$ & 0.731 & 0.191 & 0.631 & 5.538 & 0.69 \\
& $0.67<x_{F} \leq 1.00$ & 0.804 & 0.186 & 0.728 & 5.699 & 0.83 \\
\hline
\end{tabular}

Table C.5: Average values of kinematic variables of Roadset 62 data. 


\begin{tabular}{ccccccc}
\hline Target & $x_{F}$ bin & $\left\langle x_{1}\right\rangle$ & $\left\langle x_{2}\right\rangle$ & $\left\langle x_{F}\right\rangle$ & $\langle$ mass $\rangle$ & $\left\langle p_{T}\right\rangle$ \\
\hline Fe & $0.00<x_{F} \leq 0.30$ & 0.474 & 0.281 & 0.223 & 5.395 & 0.78 \\
& $0.30<x_{F} \leq 0.41$ & 0.532 & 0.218 & 0.358 & 5.038 & 0.73 \\
& $0.41<x_{F} \leq 0.50$ & 0.595 & 0.197 & 0.453 & 5.045 & 0.78 \\
& $0.50<x_{F} \leq 0.60$ & 0.659 & 0.187 & 0.541 & 5.176 & 0.76 \\
& $0.60<x_{F} \leq 0.67$ & 0.729 & 0.188 & 0.630 & 5.478 & 0.75 \\
& $0.67<x_{F} \leq 1.00$ & 0.798 & 0.190 & 0.718 & 5.738 & 0.76 \\
\hline $\mathrm{C}$ & $0.00<x_{F} \leq 0.30$ & 0.474 & 0.283 & 0.220 & 5.416 & 0.77 \\
& $0.30<x_{F} \leq 0.41$ & 0.536 & 0.219 & 0.361 & 5.060 & 0.74 \\
& $0.41<x_{F} \leq 0.50$ & 0.594 & 0.195 & 0.453 & 5.015 & 0.75 \\
& $0.50<x_{F} \leq 0.60$ & 0.661 & 0.188 & 0.542 & 5.195 & 0.76 \\
& $0.60<x_{F} \leq 0.67$ & 0.730 & 0.186 & 0.631 & 5.437 & 0.81 \\
& $0.67<x_{F} \leq 1.00$ & 0.792 & 0.185 & 0.715 & 5.669 & 0.66 \\
\hline $\mathrm{W}$ & $0.00<x_{F} \leq 0.30$ & 0.476 & 0.282 & 0.224 & 5.415 & 0.81 \\
& $0.30<x_{F} \leq 0.41$ & 0.530 & 0.214 & 0.358 & 4.975 & 0.76 \\
& $0.41<x_{F} \leq 0.50$ & 0.593 & 0.195 & 0.452 & 5.021 & 0.75 \\
& $0.50<x_{F} \leq 0.60$ & 0.665 & 0.192 & 0.544 & 5.262 & 0.80 \\
& $0.60<x_{F} \leq 0.67$ & 0.729 & 0.185 & 0.631 & 5.403 & 0.80 \\
& $0.67<x_{F} \leq 1.00$ & 0.802 & 0.192 & 0.723 & 5.781 & 0.79 \\
\hline
\end{tabular}

Table C.6: Average values of kinematic variables of Roadset 67 data. 


\section{C.2 Rate-dependent Tracking Efficiency Correction}

\begin{tabular}{lcccccc} 
Run-II & \multicolumn{7}{l}{} \\
\hline$x_{F}$ bin & $\left\langle I_{F e}^{C}\right\rangle$ & $C_{F e}^{c o r}$ & $\left\langle I_{C}^{C}\right\rangle$ & $C_{C}^{c o r}$ & $\left\langle I_{W}^{C}\right\rangle$ & $C_{W}^{c o r}$ \\
\hline $0.00<x_{F} \leq 0.30$ & 21172.7 & 1.309 & 19708.0 & 1.313 & 21436.0 & 1.189 \\
$0.30<x_{F} \leq 0.41$ & 24316.8 & 1.242 & 23138.8 & 1.282 & 24980.9 & 1.175 \\
$0.41<x_{F} \leq 0.50$ & 22834.4 & 1.161 & 23849.1 & 1.192 & 23840.6 & 1.230 \\
$0.50<x_{F} \leq 0.60$ & 23180.2 & 1.107 & 23081.7 & 1.100 & 22318.5 & 1.181 \\
$0.60<x_{F} \leq 0.67$ & 26612.6 & 1.099 & 20693.9 & 1.059 & 23240.3 & 1.063 \\
$0.67<x_{F} \leq 1.00$ & 9241.9 & 1.041 & 24287.6 & 1.090 & 24008.3 & 1.133 \\
\hline
\end{tabular}

\begin{tabular}{lcccccc} 
Roadset 62 & \multicolumn{7}{l}{} \\
\hline$x_{F}$ bin & $\left\langle I_{F e}^{C}\right\rangle$ & $C_{F e}^{\text {cor }}$ & $\left\langle I_{C}^{C}\right\rangle$ & $C_{C}^{\text {cor }}$ & $\left\langle I_{W}^{C}\right\rangle$ & $C_{W}^{\text {cor }}$ \\
\hline $0.00<x_{F} \leq 0.30$ & 23121.7 & 1.429 & 22631.8 & 1.391 & 21832.7 & 1.419 \\
$0.30<x_{F} \leq 0.41$ & 24788.4 & 1.306 & 24567.1 & 1.265 & 22981.1 & 1.304 \\
$0.41<x_{F} \leq 0.50$ & 23675.6 & 1.226 & 24771.6 & 1.273 & 24968.3 & 1.177 \\
$0.50<x_{F} \leq 0.60$ & 22069.4 & 1.192 & 24959.9 & 1.256 & 24931.4 & 1.280 \\
$0.60<x_{F} \leq 0.67$ & 25145.6 & 1.114 & 22242.6 & 1.124 & 27114.2 & 1.157 \\
$0.67<x_{F} \leq 1.00$ & 21252.5 & 1.140 & 33240.5 & 1.157 & 22249.1 & 1.132 \\
\hline
\end{tabular}

\begin{tabular}{lcccccc} 
Roadset 67 & \multicolumn{7}{l}{} \\
\hline$x_{F}$ bin & $\left\langle I_{F e}^{C}\right\rangle$ & $C_{F e}^{\text {cor }}$ & $\left\langle I_{C}^{C}\right\rangle$ & $C_{C}^{\text {cor }}$ & $\left\langle I_{W}^{C}\right\rangle$ & $C_{W}^{\text {cor }}$ \\
\hline $0.00<x_{F} \leq 0.30$ & 22893.9 & 1.503 & 23442.3 & 1.457 & 24341.9 & 1.518 \\
$0.30<x_{F} \leq 0.41$ & 25003.2 & 1.430 & 25368.7 & 1.345 & 25086.2 & 1.398 \\
$0.41<x_{F} \leq 0.50$ & 25387.9 & 1.265 & 26183.6 & 1.346 & 25426.3 & 1.307 \\
$0.50<x_{F} \leq 0.60$ & 25530.1 & 1.288 & 25223.1 & 1.251 & 25592.7 & 1.294 \\
$0.60<x_{F} \leq 0.67$ & 24164.8 & 1.293 & 27616.6 & 1.298 & 27468.7 & 1.267 \\
$0.67<x_{F} \leq 1.00$ & 25318.6 & 1.234 & 24657.0 & 1.206 & 27244.8 & 1.258 \\
\hline
\end{tabular}

Table C.7: Average chamber intensity and $C^{c o r}$. 


\section{Run-II}

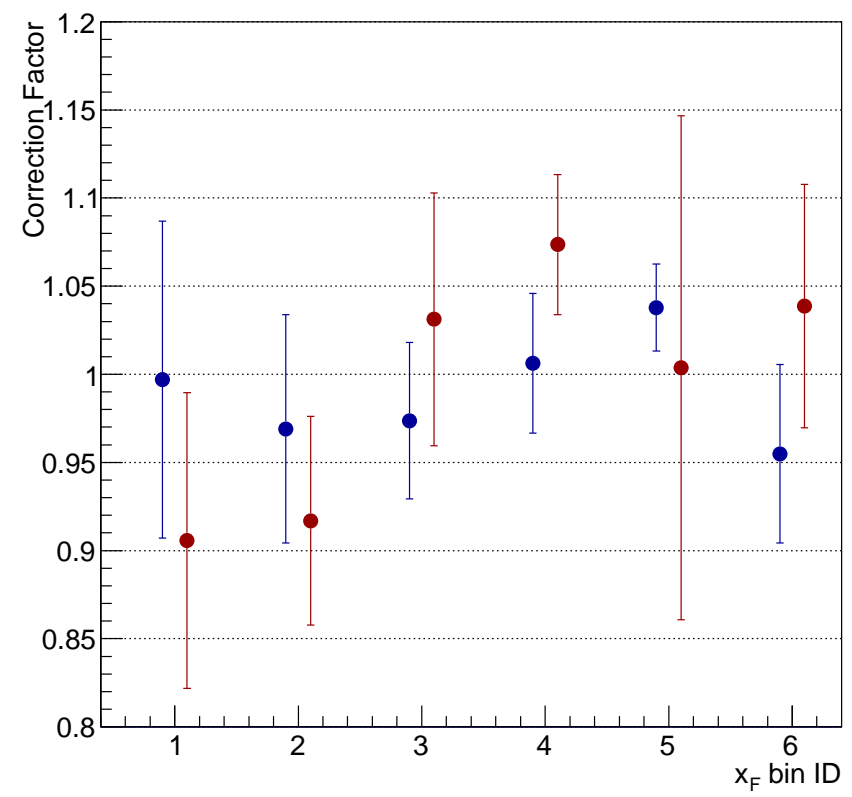

Figure C.1: Correction factor $F_{A / C}^{R D}$ for Run-II . The blue solid circles are the ones for $\mathrm{Fe} / \mathrm{C}$ and the red solid circles are for $\mathrm{W} / \mathrm{C}$.

\begin{tabular}{lccc}
$\mathbf{F e} / \mathbf{C}$ & \multicolumn{3}{c}{} \\
\hline$x_{F}$ bin & $F_{F e / C}^{R D}$ & $\delta F_{F e / C}^{\text {sys-RDfit }}$ & $\delta F_{F e / C}^{\text {sys-RDT }}$ \\
\hline $0.00<x_{F} \leq 0.30$ & 0.997 & 0.036 & 0.082 \\
$0.30<x_{F} \leq 0.41$ & 0.969 & 0.050 & 0.041 \\
$0.41<x_{F} \leq 0.50$ & 0.974 & 0.036 & 0.026 \\
$0.50<x_{F} \leq 0.60$ & 1.006 & 0.034 & 0.021 \\
$0.60<x_{F} \leq 0.67$ & 1.038 & 0.018 & 0.017 \\
$0.67<x_{F} \leq 1.00$ & 0.955 & 0.015 & 0.048 \\
\hline & & & \\
$\mathbf{W} / \mathbf{C}$ & & & \\
\hline$x_{F}$ bin & $F_{W / C}^{R D}$ & $\delta F_{W / C}^{s y s-R D f i t}$ & $\delta F_{W / C}^{s y s-R D T}$ \\
\hline $0.00<x_{F} \leq 0.30$ & 0.906 & 0.051 & 0.066 \\
$0.30<x_{F} \leq 0.41$ & 0.917 & 0.048 & 0.034 \\
$0.41<x_{F} \leq 0.50$ & 1.031 & 0.062 & 0.036 \\
$0.50<x_{F} \leq 0.60$ & 1.074 & 0.026 & 0.030 \\
$0.60<x_{F} \leq 0.67$ & 1.004 & 0.142 & 0.016 \\
$0.67<x_{F} \leq 1.00$ & 1.039 & 0.057 & 0.038 \\
\hline
\end{tabular}

Table C.8: Correction factor $F_{A / C}^{R D}$ for Run-II . 
Roadset 62

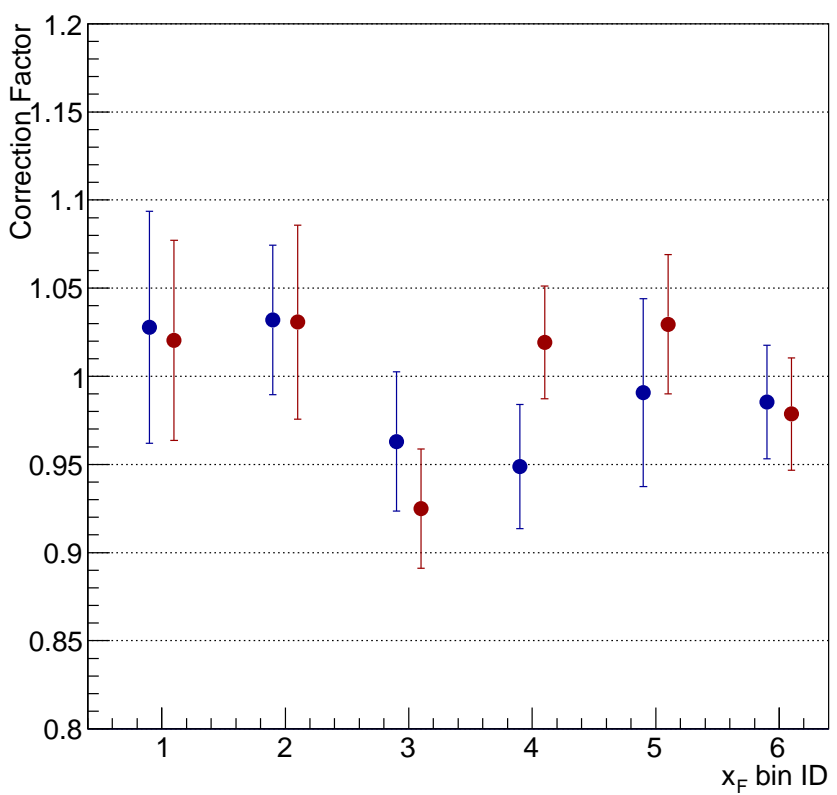

Figure C.2: Correction factor $F_{A / C}^{R D}$ for Roadset 62 . The blue solid circles are the ones for Fe/C and the red solid circles are for W/C.

\begin{tabular}{lccc}
$\mathbf{F e} / \mathbf{C}$ & \multicolumn{3}{c}{} \\
\hline$x_{F}$ bin & $F_{F e / C}^{R D}$ & $\delta F_{F e / C}^{\text {sys-RDfit }}$ & $\delta F_{F e / C}^{\text {sys-RDT }}$ \\
\hline $0.00<x_{F} \leq 0.30$ & 1.028 & 0.063 & 0.020 \\
$0.30<x_{F} \leq 0.41$ & 1.032 & 0.040 & 0.013 \\
$0.41<x_{F} \leq 0.50$ & 0.963 & 0.037 & 0.013 \\
$0.50<x_{F} \leq 0.60$ & 0.949 & 0.032 & 0.014 \\
$0.60<x_{F} \leq 0.67$ & 0.991 & 0.053 & 0.009 \\
$0.67<x_{F} \leq 1.00$ & 0.985 & 0.031 & 0.007 \\
\hline & & & \\
$\mathbf{W} / \mathbf{C}$ & & & \\
\hline$x_{F}$ bin & $F_{W / C}^{R D}$ & $\delta F_{W / C}^{s y s-R D f i t}$ & $\delta F_{W / C}^{s y s-R D T}$ \\
\hline $0.00<x_{F} \leq 0.30$ & 1.020 & 0.051 & 0.025 \\
$0.30<x_{F} \leq 0.41$ & 1.031 & 0.052 & 0.018 \\
$0.41<x_{F} \leq 0.50$ & 0.925 & 0.032 & 0.012 \\
$0.50<x_{F} \leq 0.60$ & 1.019 & 0.029 & 0.014 \\
$0.60<x_{F} \leq 0.67$ & 1.030 & 0.038 & 0.009 \\
$0.67<x_{F} \leq 1.00$ & 0.979 & 0.031 & 0.007 \\
\hline
\end{tabular}

Table C.9: Correction factor $F_{A / C}^{R D}$ for Roadset 62 . 
Roadset 67

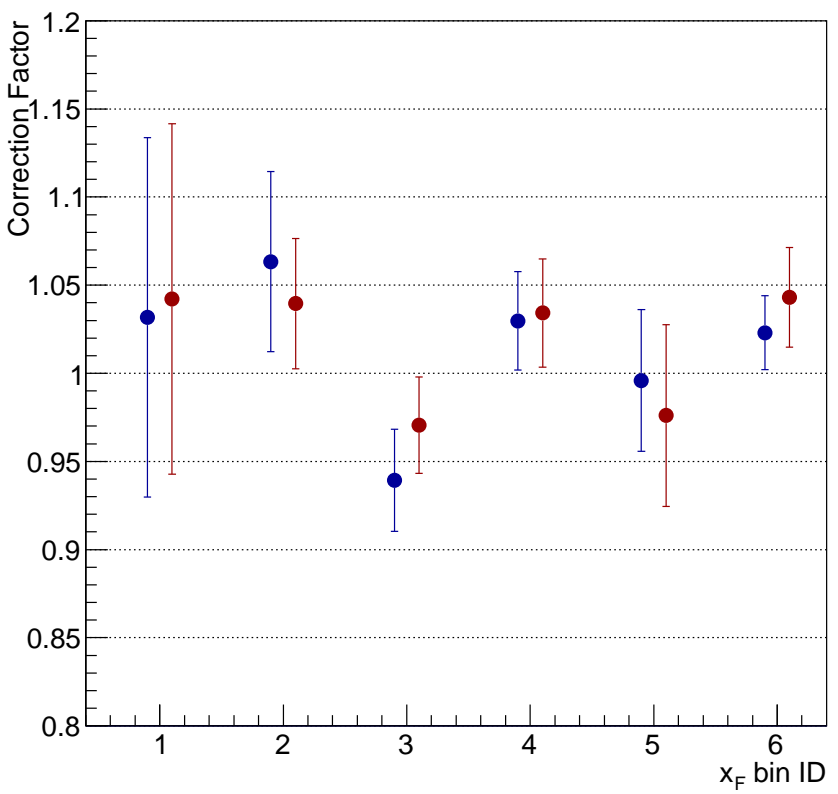

Figure C.3: Correction factor $F_{A / C}^{R D}$ for Roadset 67 . The blue solid circles are the ones for Fe/C and the red solid circles are for $\mathrm{W} / \mathrm{C}$.

\begin{tabular}{lccc}
$\mathbf{F e} / \mathbf{C}$ & \multicolumn{3}{c}{} \\
\hline$x_{F}$ bin & $F_{F e / C}^{R D}$ & $\delta F_{F e / C}^{\text {sys-RDfit }}$ & $\delta F_{F e / C}^{\text {sys-RDT }}$ \\
\hline $0.00<x_{F} \leq 0.30$ & 1.032 & 0.095 & 0.036 \\
$0.30<x_{F} \leq 0.41$ & 1.063 & 0.046 & 0.023 \\
$0.41<x_{F} \leq 0.50$ & 0.939 & 0.025 & 0.014 \\
$0.50<x_{F} \leq 0.60$ & 1.030 & 0.024 & 0.014 \\
$0.60<x_{F} \leq 0.67$ & 0.996 & 0.037 & 0.016 \\
$0.67<x_{F} \leq 1.00$ & 1.023 & 0.010 & 0.018 \\
\hline & & & \\
$\mathbf{W} / \mathbf{C}$ & & & \\
\hline$x_{F}$ bin & $F_{W / C}^{R D}$ & $\delta F_{W / C}^{s y s-R D f i t}$ & $\delta F_{W / C}^{s y s-R D T}$ \\
\hline $0.00<x_{F} \leq 0.30$ & 1.042 & 0.093 & 0.034 \\
$0.30<x_{F} \leq 0.41$ & 1.040 & 0.028 & 0.024 \\
$0.41<x_{F} \leq 0.50$ & 0.971 & 0.022 & 0.017 \\
$0.50<x_{F} \leq 0.60$ & 1.034 & 0.026 & 0.016 \\
$0.60<x_{F} \leq 0.67$ & 0.976 & 0.050 & 0.012 \\
$0.67<x_{F} \leq 1.00$ & 1.043 & 0.022 & 0.018 \\
\hline
\end{tabular}

Table C.10: Correction factor $F_{A / C}^{R D}$ for Roadset 67. 

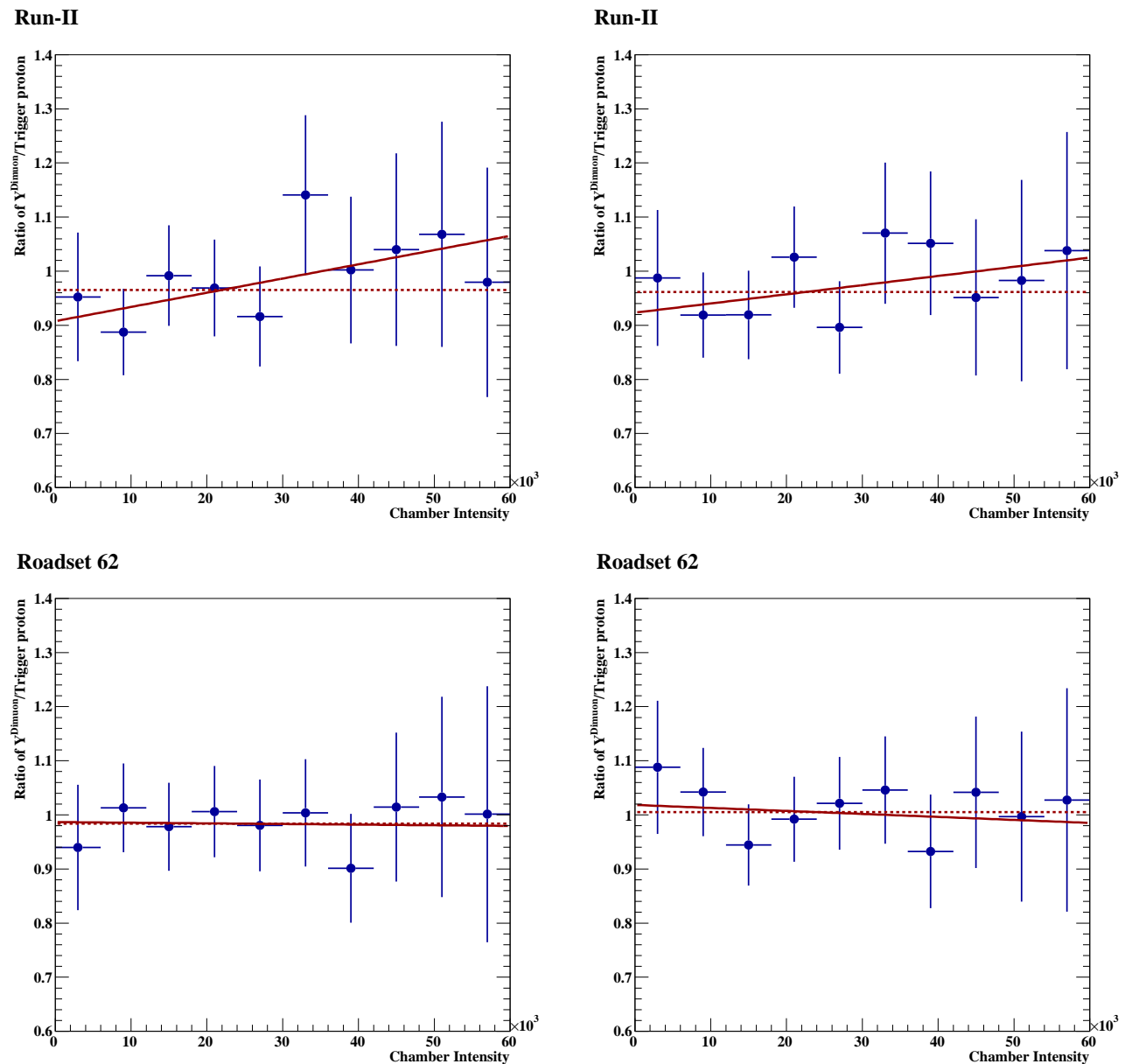

Roadset 62
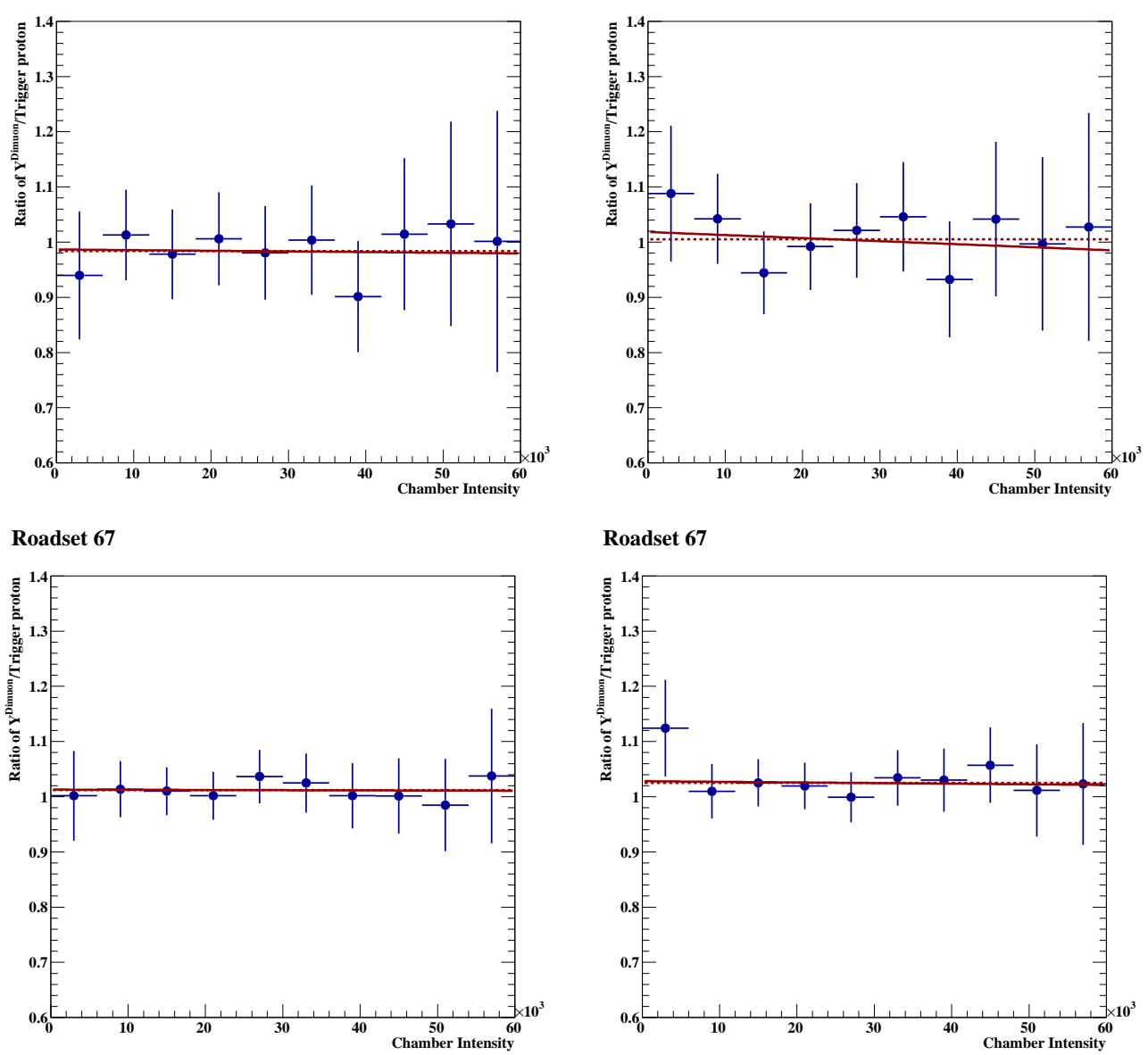

Figure C.4: Corrected ratios of dimuon yield per trigger proton for each data set. The left plot is $\mathrm{Fe} / \mathrm{C}$ and the right plot is $\mathrm{W} / \mathrm{C}$. The result of the constant fit is shown by the dashed line, and the result of the linear fit is shown by the solid line. The rather large error bar is due to the incorporation of the uncertainty of the rate-dependence correction. 


\begin{tabular}{lcc}
\hline Data set & $\delta R_{F e / C}^{\text {sys-RDremain }}$ & $\delta R_{W / C}^{\text {sys-RDremain }}$ \\
\hline Run-II & 0.074 & 0.061 \\
Roadset 62 & 0.040 & 0.048 \\
Roadset 67 & 0.017 & 0.036 \\
\hline
\end{tabular}

Table C.11: Estimated systematic error from remaining rate dependence.

\section{C.3 $R_{p A}\left(x_{F}\right)$ from Each Dataset}

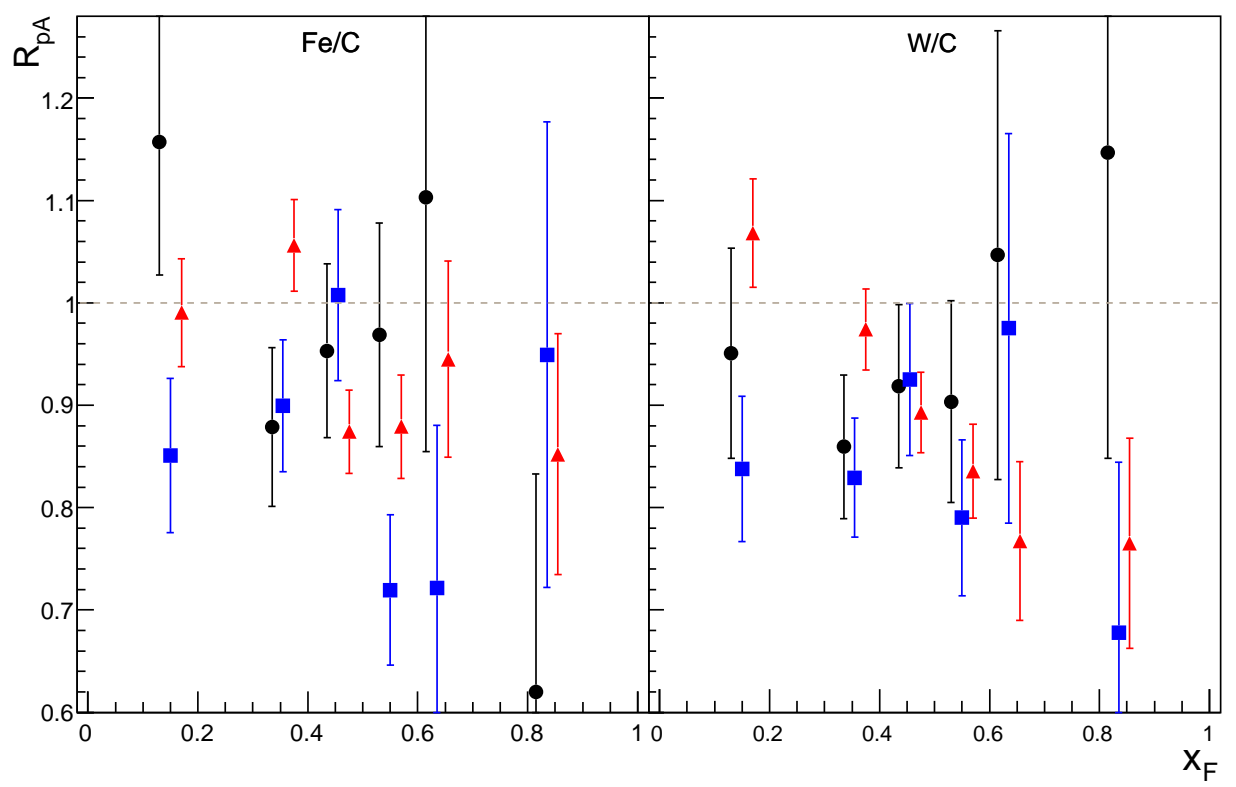

Figure C.5: $R_{p A}\left(x_{F}\right)$ acquired from each dataset. Only the statistical errors are shown. The black circles are results of Run-II , the blue squares are of Roadset 62, and red triangles are of Roadset 67. 


\begin{tabular}{lcccccc}
$\mathbf{F e} / \mathbf{C}$ & \multicolumn{7}{c}{} \\
\hline$x_{F}$ bin & $R_{p A}$ & $\sigma_{\text {stat }}$ & $\sigma_{\text {sys }}^{\text {BGfit }}$ & $\sigma_{\text {sys }}^{\text {RDit }}$ & $\sigma_{\text {sys }}^{\text {RDI }}$ & $\sigma_{\text {sys }}^{\text {RDemain }}$ \\
\hline $0.00<x_{F} \leq 0.30$ & 1.157 & 0.130 & 0.007 & 0.042 & 0.095 & 0.086 \\
$0.00<x_{F} \leq 0.30$ & 0.879 & 0.078 & 0.005 & 0.044 & 0.036 & 0.065 \\
$0.00<x_{F} \leq 0.30$ & 0.953 & 0.085 & 0.004 & 0.034 & 0.024 & 0.070 \\
$0.00<x_{F} \leq 0.30$ & 0.969 & 0.109 & 0.005 & 0.033 & 0.020 & 0.072 \\
$0.00<x_{F} \leq 0.30$ & 1.103 & 0.248 & 0.008 & 0.020 & 0.019 & 0.082 \\
$0.00<x_{F} \leq 0.30$ & 0.620 & 0.213 & 0.008 & 0.009 & 0.030 & 0.046 \\
\hline
\end{tabular}

\begin{tabular}{lcccccc}
$\mathbf{W} / \mathbf{C}$ & \multicolumn{7}{l}{} \\
\hline$x_{F}$ bin & $R_{p A}$ & $\sigma_{\text {stat }}$ & $\sigma_{\text {sys }}^{\text {BGfit }}$ & $\sigma_{\text {sys }}^{\text {RDit }}$ & $\sigma_{\text {sys }}^{\text {RDI }}$ & $\sigma_{\text {sys }}^{\text {RDemain }}$ \\
\hline $0.30<x_{F} \leq 0.41$ & 0.951 & 0.102 & 0.006 & 0.049 & 0.063 & 0.058 \\
$0.30<x_{F} \leq 0.41$ & 0.859 & 0.070 & 0.005 & 0.041 & 0.029 & 0.053 \\
$0.30<x_{F} \leq 0.41$ & 0.919 & 0.080 & 0.005 & 0.057 & 0.034 & 0.056 \\
$0.30<x_{F} \leq 0.41$ & 0.903 & 0.099 & 0.005 & 0.024 & 0.027 & 0.055 \\
$0.30<x_{F} \leq 0.41$ & 1.047 & 0.219 & 0.007 & 0.149 & 0.017 & 0.064 \\
$0.30<x_{F} \leq 0.41$ & 1.147 & 0.298 & 0.010 & 0.066 & 0.044 & 0.070 \\
\hline
\end{tabular}

Table C.12: $R_{p A}$ values and the corresponding errors for Run-II .

\begin{tabular}{lcccccc}
$\mathbf{F e} / \mathbf{C}$ & \multicolumn{7}{c}{} \\
\hline$x_{F}$ bin & $R_{p A}$ & $\sigma_{\text {stat }}$ & $\sigma_{\text {sys }}^{\text {BGfit }}$ & $\sigma_{\text {sys }}^{\text {RDit }}$ & $\sigma_{\text {sys }}^{\text {RDI }}$ & $\sigma_{\text {sys }}^{\text {RDemain }}$ \\
\hline $0.00<x_{F} \leq 0.30$ & 0.851 & 0.075 & 0.003 & 0.053 & 0.017 & 0.034 \\
$0.00<x_{F} \leq 0.30$ & 0.899 & 0.065 & 0.004 & 0.036 & 0.012 & 0.036 \\
$0.00<x_{F} \leq 0.30$ & 1.008 & 0.084 & 0.005 & 0.038 & 0.013 & 0.040 \\
$0.00<x_{F} \leq 0.30$ & 0.719 & 0.073 & 0.003 & 0.023 & 0.010 & 0.028 \\
$0.00<x_{F} \leq 0.30$ & 0.722 & 0.159 & 0.005 & 0.038 & 0.006 & 0.029 \\
$0.00<x_{F} \leq 0.30$ & 0.949 & 0.227 & 0.005 & 0.030 & 0.007 & 0.038 \\
\hline
\end{tabular}

\begin{tabular}{lcccccc}
$\mathbf{W} / \mathbf{C}$ & \multicolumn{7}{l}{} \\
\hline$x_{F}$ bin & $R_{p A}$ & $\sigma_{\text {stat }}$ & $\sigma_{\text {sys }}^{\text {BGfit }}$ & $\sigma_{\text {sys }}^{\text {RDit }}$ & $\sigma_{\text {sys }}^{\text {RDI }}$ & $\sigma_{\text {sys }}^{\text {RDemain }}$ \\
\hline $0.30<x_{F} \leq 0.41$ & 0.838 & 0.071 & 0.003 & 0.043 & 0.021 & 0.040 \\
$0.30<x_{F} \leq 0.41$ & 0.829 & 0.058 & 0.004 & 0.043 & 0.015 & 0.040 \\
$0.30<x_{F} \leq 0.41$ & 0.925 & 0.074 & 0.005 & 0.029 & 0.011 & 0.044 \\
$0.30<x_{F} \leq 0.41$ & 0.790 & 0.076 & 0.004 & 0.023 & 0.011 & 0.038 \\
$0.30<x_{F} \leq 0.41$ & 0.975 & 0.190 & 0.006 & 0.037 & 0.009 & 0.047 \\
$0.30<x_{F} \leq 0.41$ & 0.678 & 0.167 & 0.004 & 0.021 & 0.004 & 0.032 \\
\hline
\end{tabular}

Table C.13: $R_{p A}$ values and the corresponding errors for Roadset 62 . 


\begin{tabular}{lcccccc}
$\mathbf{F e} / \mathbf{C}$ & \multicolumn{1}{l}{} \\
\hline$x_{F}$ bin & $R_{p A}$ & $\sigma_{\text {stat }}$ & $\sigma_{\text {sys }}^{\text {BGfit }}$ & $\sigma_{\text {sys }}^{\text {Rfit }}$ & $\sigma_{\text {sys }}^{\text {RDI }}$ & $\sigma_{\text {sys }}^{\text {RDemain }}$ \\
\hline $0.00<x_{F} \leq 0.30$ & 0.991 & 0.053 & 0.003 & 0.094 & 0.036 & 0.017 \\
$0.00<x_{F} \leq 0.30$ & 1.056 & 0.045 & 0.003 & 0.048 & 0.024 & 0.018 \\
$0.00<x_{F} \leq 0.30$ & 0.874 & 0.041 & 0.003 & 0.022 & 0.012 & 0.015 \\
$0.00<x_{F} \leq 0.30$ & 0.879 & 0.050 & 0.002 & 0.021 & 0.013 & 0.015 \\
$0.00<x_{F} \leq 0.30$ & 0.945 & 0.096 & 0.003 & 0.035 & 0.015 & 0.016 \\
$0.00<x_{F} \leq 0.30$ & 0.852 & 0.118 & 0.004 & 0.009 & 0.016 & 0.014 \\
\hline
\end{tabular}

\begin{tabular}{lcccccc}
$\mathbf{W} / \mathbf{C}$ & \multicolumn{7}{l}{} \\
\hline$x_{F}$ bin & $R_{p A}$ & $\sigma_{\text {stat }}$ & $\sigma_{\text {sys }}^{\text {BGfit }}$ & $\sigma_{\text {sys }}^{\text {RDit }}$ & $\sigma_{\text {sys }}^{\text {RDI }}$ & $\sigma_{\text {sys }}^{\text {RDremain }}$ \\
\hline $0.30<x_{F} \leq 0.41$ & 1.068 & 0.053 & 0.003 & 0.100 & 0.036 & 0.038 \\
$0.30<x_{F} \leq 0.41$ & 0.974 & 0.040 & 0.003 & 0.027 & 0.023 & 0.035 \\
$0.30<x_{F} \leq 0.41$ & 0.893 & 0.039 & 0.003 & 0.019 & 0.015 & 0.032 \\
$0.30<x_{F} \leq 0.41$ & 0.836 & 0.046 & 0.002 & 0.022 & 0.013 & 0.030 \\
$0.30<x_{F} \leq 0.41$ & 0.767 & 0.077 & 0.003 & 0.038 & 0.010 & 0.027 \\
$0.30<x_{F} \leq 0.41$ & 0.765 & 0.103 & 0.003 & 0.017 & 0.014 & 0.027 \\
\hline
\end{tabular}

Table C.14: $R_{p A}$ values and the corresponding errors for Roadset 67. 


\section{C.4 Combined $R_{p A}\left(x_{F}\right)$ Result}

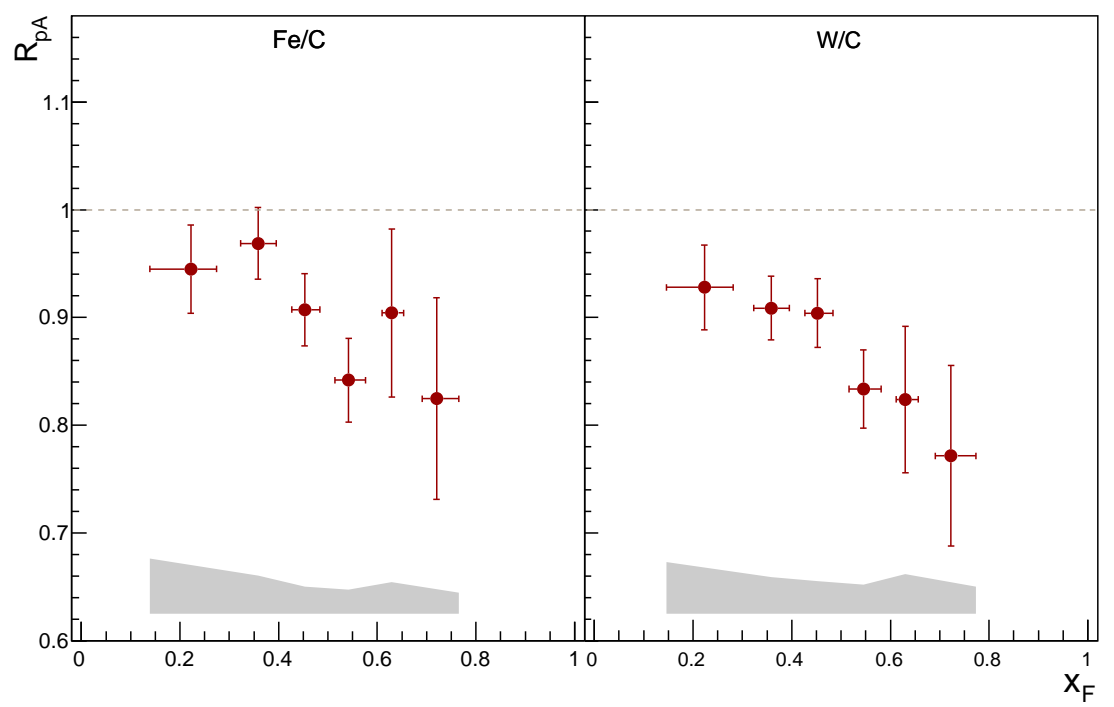

Figure C.6: The combined $R_{p A}\left(x_{F}\right)$ result. The statistical errors are plotted along with the data points and the corresponding systematic errors are presented as the grey band.

\begin{tabular}{llll}
$\mathbf{F e} / \mathbf{C}$ & & & \\
\hline$x_{F}$ & $\overline{R_{p A}}$ & $\pm \sigma_{\text {stat }}$ & $\pm \sigma_{\text {sys }}$ \\
\hline $0.22[+0.05,-0.08]$ & 0.945 & \pm 0.041 & \pm 0.051 \\
$0.36[+0.04,-0.03]$ & 0.969 & \pm 0.033 & \pm 0.035 \\
$0.45[+0.03,-0.03]$ & 0.907 & \pm 0.034 & \pm 0.025 \\
$0.54[+0.03,-0.03]$ & 0.842 & \pm 0.039 & \pm 0.022 \\
$0.63[+0.02,-0.02]$ & 0.904 & \pm 0.078 & \pm 0.029 \\
$0.72[+0.04,-0.03]$ & 0.825 & \pm 0.094 & \pm 0.020 \\
\hline & & &
\end{tabular}

\begin{tabular}{llll}
$\mathbf{W} / \mathbf{C}$ & & & \\
\hline$x_{F}$ & $\overline{R_{p A}}$ & $\pm \sigma_{\text {stat }}$ & $\pm \sigma_{\text {sys }}$ \\
\hline $0.22[+0.06,-0.08]$ & 0.928 & \pm 0.039 & \pm 0.048 \\
$0.36[+0.04,-0.04]$ & 0.909 & \pm 0.030 & \pm 0.034 \\
$0.45[+0.03,-0.03]$ & 0.904 & \pm 0.032 & \pm 0.030 \\
$0.55[+0.04,-0.03]$ & 0.834 & \pm 0.037 & \pm 0.027 \\
$0.63[+0.03,-0.02]$ & 0.824 & \pm 0.068 & \pm 0.037 \\
$0.72[+0.05,-0.03]$ & 0.772 & \pm 0.084 & \pm 0.025 \\
\hline
\end{tabular}

Table C.15: Combined $R_{p A}$ values and the corresponding errors. 